\title{
Non-Gaussianity and the Cosmic Microwave Background Anisotropies
}

\author{
N. Bartolo, ${ }^{1,2}$ S. Matarrese, ${ }^{1,2}$ and A. Riotto ${ }^{2,3}$ \\ ${ }^{1}$ Dipartimento di Fisica “G. Galilei”, Università di Padova, via Marzolo 8, 131 Padova, Italy \\ ${ }^{2}$ INFN, Sezione di Padova, via Marzolo 8, 35131 Padova, Italy \\ ${ }^{3}$ CERN, Theory Division, CH-1211 Geneva 23, Switzerland
}

Correspondence should be addressed to N. Bartolo, bartolo@pd.infn.it

Received 19 January 2010; Revised 14 June 2010; Accepted 29 June 2010

Academic Editor: Eiichiro Komatsu

Copyright $\odot 2010$ N. Bartolo et al. This is an open access article distributed under the Creative Commons Attribution License, which permits unrestricted use, distribution, and reproduction in any medium, provided the original work is properly cited.

\begin{abstract}
We review in a pedagogical way the present status of the impact of non-Gaussianity (NG) on the cosmic microwave background $(\mathrm{CMB})$ anisotropies. We first show how to set the initial conditions at second order for the CMB anisotropies when some primordial NG is present. However, there are many sources of NG in CMB anisotropies, beyond the primordial one, which can contaminate the primordial signal. We mainly focus on the NG generated from the post inflationary evolution of the $\mathrm{CMB}$ anisotropies at second order in perturbation theory at large and small angular scales, such as the ones generated at the recombination epoch. We show how to derive the equations to study the second-order CMB anisotropies and provide analytical computations to evaluate their contamination to primordial NG (complemented with numerical examples). We also offer a brief summary of other secondary effects. This paper requires basic knowledge of the theory of cosmological perturbations at the linear level.
\end{abstract}

\section{Introduction}

Cosmic microwave background (CMB) anisotropies play a special role in cosmology, as they allow an accurate determination of cosmological parameters and may provide a unique probe of the physics of the early universe and in particular of the processes that gave origin to the primordial perturbations.

Cosmological inflation [1] is nowadays considered the dominant paradigm for the generation of the initial seeds for structure formation. In the inflationary picture, the primordial cosmological perturbations are created from quantum fluctuations "redshifted" out of the horizon during an early period of accelerated expansion of the universe, where they remain "frozen". They are observable through CMB temperature anisotropies (and polarization) and the large-scale clustering properties of the matter distribution in the Universe.

This picture has recently received further spectacular confirmations from the results of the wilkinson microwave anisotropy probe (WMAP) five year set of data [2]. Since the observed cosmological perturbations are of the order of $10^{-5}$, one might think that first-order perturbation theory will be adequate for all comparisons with observations. This might not be the case, though. Present $[2,3]$ and future experiments [4] may be sensitive to the nonlinearities of the cosmological perturbations at the level of second- or higher-order perturbation theory. The detection of these nonlinearities through the non-Gaussianity (NG) in the CMB [5] has become one of the primary experimental targets.

There is one fundamental reason why a positive detection of NG is so relevant: it might help in discriminating among the various mechanisms for the generation of the cosmological perturbations. Indeed, various models of inflation, firmly rooted in modern particle physics theory, predict a significant amount of primordial NG generated either during or immediately after inflation when the comoving curvature perturbation becomes constant on superhorizon scales [5]. While standard single-field models of slow-roll inflation $[6,7]$ and-in general-two (multi)field $[8,9]$ models of inflation predict a tiny level of NG, "curvaton"-type 
models, in which a significant contribution to the curvature perturbation is generated after the end of slow-roll inflation by the perturbation in a field which has a negligible effect on inflation, may predict a high level of NG [10]. Alternatives to the curvaton model are models where a curvature perturbation mode is generated by an inhomogeneity in the decay rate [11-13], the mass [14], or the interaction rate [15] of the particles responsible for the reheating after inflation. Other opportunities for generating the curvature perturbations occur at the end of inflation [16-18], during preheating [19-21], and at a phase-transition producing cosmic strings [22]. Also, within single-field models of inflation, a high level of NG can be generated breaking the standard conditions of canonical kinetic terms and initially vacuum states: for example, this is the case of Dirac-borninfeld (DBI) models of inflation [23], and initially excited states, respectively [24]. For every scenario, there exists a well defined prediction for the strength of NG and its shape $[25,26]$ as a function of the parameters.

Statistics like the bispectrum and the trispectrum of the $\mathrm{CMB}$ can then be used to assess the level of primordial NG (and possibly its shape) on various cosmological scales and to discriminate it from the one induced by secondary anisotropies and systematic effects [5, 27-30]. A positive detection of a primordial $\mathrm{NG}$ in the $\mathrm{CMB}$ at some level might therefore confirm and/or rule out a whole class of mechanisms by which the cosmological perturbations have been generated.

Despite the importance of evaluating the impact of primordial NG in a crucial observable like the CMB anisotropy, the vast majority of the literature has been devoted to the computation of the bispectrum of either the comoving curvature perturbation or the gravitational potential on large scales within given inflationary models. These, however, are not the physical quantities which are observed. One should instead provide a full prediction for the secondorder radiation transfer function. A preliminary step towards this goal has been taken in [31] (see also [32-37]) where the full second-order radiation transfer function for the $\mathrm{CMB}$ anisotropies on large angular scales in a flat universe filled with matter and cosmological constant was computed, including the second-order generalization of the Sachs-Wolfe effect, both the early and late integrated Sachs-Wolfe (ISW) effects and the contribution of the second order tensor modes. (A similar computation of the CMB anisotropies up to third-order from gravitational perturbations has been performed in [38], which is particularly relevant to provide a complete theoretical prediction for cubic nonlinearities characterizing the level of $\mathrm{NG}$ in the $\mathrm{CMB}$ through the connected four-point correlation function (trispectrum) [27, 28].)

There are many sources of NG in CMB anisotropies, beyond the primordial one. The most relevant sources are the so-called secondary anisotropies, which arise after the last scattering epoch. These anisotropies can be divided into two categories: scattering secondaries, when the CMB photons scatter with electrons along the line of sight, and gravitational secondaries when effects are mediated by gravity [39]. Among the scattering secondaries we may list the thermal Sunyaev-Zeldovich effect, where hot electrons in clusters transfer energy to the CMB photons, the kinetic Sunyaev-Zeldovich effect produced by the bulk motion of the electrons in clusters, the Ostriker-Vishniac effect, produced by bulk motions modulated by linear density perturbations, and effects due to reionization processes. The scattering secondaries are most significant on small angular scales as density inhomogeneities, bulk and thermal motions grow and become sizeable on small length-scales when structure formation proceeds.

Gravitational secondaries arise from the change in energy of photons when the gravitational potential is timedependent, the ISW effect, and gravitational lensing. At late times, when the Universe becomes dominated by the dark energy, the gravitational potential on linear scales starts to decay, causing the ISW effect mainly on large angular scales. Other secondaries that result from a timedependent potential are the Rees-Sciama effect, produced during the matter-dominated epoch by the time evolution of the potential on nonlinear scales.

The fact that the potential never grows appreciably means that most second order effects created by gravitational secondaries are generically small compared to those created by scattering ones. However, when a photon propagates from the last scattering to us, its path may be deflected because of the gravitational lensing. This effect does not create anisotropies, but only modifies existing ones. Since photons with large wavenumbers $k$ are lensed over many regions $(\sim k / H$, where $H$ is the Hubble rate) along the line of sight, the corresponding second-order effect may be sizeable. The three-point function arising from the correlation of the gravitational lensing and ISW effects generated by the matter distribution along the line of sight [40, 41] and the Sunyaev-Zeldovich effect [42] are large and detectable by Planck $[43,44]$. A crucial issue is the level of contamination to the extraction of the primordial NG the secondary effects can produce. In Section 8, we briefly summarize some recent results about the level of CMB NG generated by some of these secondary effects.

Another relevant source of NG comes from the physics operating at the recombination. A naive estimate would tell that these nonlinearities are tiny being suppressed by an extra power of the gravitational potential. However, the dynamics at recombination is quite involved because all the nonlinearities in the evolution of the baryon-photon fluid at recombination and the ones coming from general relativity should be accounted for. This complicated dynamics might lead to unexpected suppressions or enhancements of the NG at recombination. A step towards the evaluation of the three-point correlation function has been taken in [45] where some effects were taken into account in the so-called squeezed triangle limit, corresponding to the case when one wavenumber is much smaller than the other two and was outside the horizon at recombination. Referances [46, 47] (see also [48-50]) present the computation of the full system of Boltzmann equations, describing the evolution of the photon, baryon and cold dark matter (CDM) fluids, at second order and neglecting polarization. These equations allow to follow the time evolution of the $\mathrm{CMB}$ anisotropies 
at second order on all angular scales from the early epochs, when the cosmological perturbations were generated, to the present time, through the recombination era. These calculations set the stage for the computation of the full second-order radiation transfer function at all scales and for a generic set of initial conditions specifying the level of primordial non-Gaussianity. Of course, for specific effects on small angular scales like Sunyaev-Zel'dovich, gravitational lensing, and so forth, fully nonlinear calculations would provide a more accurate estimate of the resulting $\mathrm{CMB}$ anisotropy, however, as long as the leading contribution to second-order statistics like the bispectrum is concerned, second-order perturbation theory suffices.

The goal of this paper is to summarize in a pedagogical form the present status of the evaluation of the impact of NG on the CMB anisotropies. This implies first of all determining how to set the initial conditions at second order for the $\mathrm{CMB}$ anisotropy when some source of primordial NG is present. The second step will be determining how primordial NG flows on small angular scales. In this paper we will focus on the study of the second-order effects appearing at the recombination era when the CMB anisotropy is left imprinted. We will show how to derive the equations to evaluate $\mathrm{CMB}$ anisotropies, by computing the Boltzmann equations describing the evolution of the baryon-photon fluid up to second order. This permits to follow the time evolution of $\mathrm{CMB}$ anisotropies (up to second order) on all scales, from the early epoch, when the cosmological perturbations were generated, to the present time, through the recombination era. We will also provide the reader with some simplified analytical computation to evaluate the contamination of the recombination secondary effects onto the detection of primordial NG. The formalism for a more refined numerical analysis is also displayed and results for some worked examples will be also reported. The paper is mainly based on a series of papers written by the authors along the past years on the subject (with various updates) and, as such, follows both a logic and a chronological order. It requires knowledge of the theory of cosmological perturbation at the linear level (which however we summarize in the Appendices). We have tried to write the different sections in a self-contained way. Nevertheless, we alert the reader that the level of complexity increases with the number of the sections.

The paper is organized as follows. In Section 2, we provide a simple, but illuminating example to show why we do expect some NG present in the $\mathrm{CMB}$ anisotropy regardless if there is or not some primordial NG. In Section 3, we provide the reader with the necessary tools to study the dynamics at second order in the gravity sector. In Section 4, we show how to set the initial conditions for the primordial NG, while in Section 5, we provide a gaugeinvariant way to define the $\mathrm{CMB}$ temperature anisotropy at second order on large scales. In Section 6, we go to small scales and present the full procedure to compute the Boltzmann equations necessary to follow the evolution of the nonlinearities from the recombination epoch down to the present epoch. Section 7 presents some analytical solutions of the Boltzmann equations in the tight coupling limit, along the same lines of what is done at the linear level. The issue of contamination is addressed in Section 8, while in Section 9 we offer the reader with an analytical estimate of such a contamination. A more refined numerical work is presented in Section 10. Finally, in Section 11, some conclusions are given. This paper has also some hopefully useful appendices: in Appendix A the reader can find the energy-momentum tensors at second-order, Appendix B gives the solutions of Einstein equations for the perturbed fluids up to secondorder, while Appendix $\mathrm{C}$ offers the analytical solutions of the linearized Boltzmann equations in the tight coupling limit.

\section{Why Do We Expect NG in the Cosmological Perturbations?}

Before tackling the problem of interest-the computation of the cosmological perturbations at second order after the inflationary era-we first provide a simple, but insightful computation, derived from [36], which illustrates why we expect that the cosmological perturbations develop some NG even if the latter is not present at some primordial epoch. This example will help the reader to understand why the cosmological perturbations are inevitably affected by nonlinearities, beyond those arising at some primordial epoch. The reason is clear: gravity is nonlinear and it feeds this property into the cosmological perturbations during the postinflationary evolution of the universe. As gravity talks to all fluids, this transmission is inevitable. To be specific, we focus on the CMB anisotropies. We will adopt the Poisson gauge which eliminates one scalar degree of freedom from the $g_{0 i}$ component of the metric and one scalar and two vector degrees of freedom from $g_{i j}$. We will use a metric of the form (see Table 1 for the symbols used in this paper)

$$
\begin{aligned}
d s^{2}= & -e^{2 \Phi} d t^{2}+2 a(t) \omega_{i} d x^{i} d t \\
& +a^{2}(t)\left(e^{-2 \Psi} \delta_{i j}+\chi_{i j}\right) d x^{i} d x^{j},
\end{aligned}
$$

where $a(t)$ is the scale factor as a function of the cosmic time $t$, and $\omega_{i}$ and $\chi_{i j}$ are the vector and tensor perturbation modes respectively. Each metric perturbation can be expanded into a linear (first-order) and a second-order part, as for example, the gravitational potential $\Phi=\Phi^{(1)}+\Phi^{(2)} / 2$. However, in the metric (1) the choice of the exponentials greatly helps in computing the relevant expressions, and thus we will always keep them where it is convenient. From (1), one recovers at linear order the well-known longitudinal gauge while at second order, one finds $\Phi^{(2)}=\phi^{(2)}-2\left(\phi^{(1)}\right)^{2}$ and $\Psi^{(2)}=\psi^{(2)}+2\left(\psi^{(1)}\right)^{2}$ where $\phi^{(1)}, \psi^{(1)}$ and $\phi^{(2)}, \psi^{(2)}$ (with $\phi^{(1)}=\Phi^{(1)}$ and $\psi^{(1)}=\Psi^{(1)}$ ) are the first and secondorder gravitational potentials in the longitudinal (Poisson) gauge adopted in $[5,51]$ as far as scalar perturbations are concerned.

We now consider the long wavelength modes of the $\mathrm{CMB}$ anisotropies, that is, we focus on scales larger than the horizon at last-scattering. We can therefore neglect vector and tensor perturbation modes in the metric. For the vector perturbations, the reason is that they contain gradient terms being produced as nonlinear combination of scalar-modes 
and thus they will be more important on small scales (linear vector modes are not generated in standard mechanisms for cosmological perturbations, as inflation). The tensor contribution can be neglected for two reasons. First, the tensor perturbations produced from inflation on large scales give a negligible contribution to the higher-order statistics of the Sachs-Wolfe effect being of the order of (powers of) the slow-roll parameters during inflation (this holds for linear tensor modes as well as for tensor modes generated by the nonlinear evolution of scalar perturbations during inflation). Second, while on large scales the tensor modes have been proven to remain constant in time [52], when they approach the horizon they have a wavelike contribution which oscillates in time with decreasing amplitude [51].

Since we are interested in the cosmological perturbations on large scales, that is in perturbations whose wavelength is larger than the Hubble radius at last scattering, a local observer would see them in the form of a classical-possibly time-dependent-(nearly zero-momentum) homogeneous and isotropic background. Therefore, it should be possible to perform a change of coordinates in such a way as to absorb the super-Hubble modes and work with a metric of an homogeneous and isotropic Universe (plus, of course, cosmological perturbations on scale smaller than the horizon). We split the gravitational potential $\Phi$ as

$$
\Phi=\Phi_{\ell}+\Phi_{s}
$$

where $\Phi_{\ell}$ stands for the part of the gravitational potential receiving contributions only from the super-Hubble modes; $\Phi_{s}$ receives contributions only from the subhorizon modes

$$
\begin{aligned}
& \Phi_{\ell}=\int \frac{d^{3} k}{(2 \pi)^{3}} \theta(a H-k) \Phi_{\vec{k}} e^{i \vec{k} \cdot \vec{x}}, \\
& \Phi_{s}=\int \frac{d^{3} k}{(2 \pi)^{3}} \theta(k-a H) \Phi_{\vec{k}} e^{i \vec{k} \cdot \vec{x}},
\end{aligned}
$$

where $H$ is the Hubble rate computed with respect to the cosmic time, $H=\dot{a} / a$, and $\theta(x)$ is the step function. Analogous definitions hold for the other gravitational potential $\Psi$.

By construction, $\Phi_{\ell}$ and $\Psi_{\ell}$ are a collection of Fourier modes whose wavelengths are larger than the horizon length and we may safely neglect their spatial gradients. Therefore, $\Phi_{\ell}$ and $\Psi_{\ell}$ are only functions of time. This amounts to saying that we can absorb the large-scale perturbations in the metric (1) by the following redefinitions:

$$
\begin{gathered}
d \bar{t}=e^{\Phi_{\ell}} d t, \\
\bar{a}=a e^{-\Psi_{\ell}} .
\end{gathered}
$$

The new metric describes a homogeneous and isotropic Universe

$$
d s^{2}=-d \bar{t}^{2}+\bar{a}^{2} \delta_{i j} d x^{i} d x^{j},
$$

where for simplicity we have not included the subhorizon modes. On superhorizon scales one can regard the Universe as a collection of regions of size of the Hubble radius evolving like unperturbed patches with metric (5) [52].
Let us now go back to the quantity we are interested in, namely the anisotropies of the CMB as measured today by an observer $\mathcal{O}$. If she/he is interested in the $\mathrm{CMB}$ anisotropies at large scales, the effect of super-Hubble modes is encoded in the metric (5). During their travel from the last scattering surface-to be considered as the emitter point $\&$ - to the observer, the CMB photons suffer a redshift determined by the ratio of the emitted frequency $\bar{\omega}_{\mathcal{E}}$ to the observed one $\bar{\omega}_{\mathcal{O}}$

$$
\bar{T}_{\mathcal{O}}=\bar{T}_{\mathcal{E}} \frac{\bar{\omega}_{\mathcal{O}}}{\bar{\omega}_{\mathcal{E}}}
$$

where $\bar{T}_{\mathcal{O}}$ and $\bar{T}_{\mathcal{E}}$ are the temperatures at the observer point and at the last scattering surface, respectively.

What is then the temperature anisotropy measured by the observer? (6) shows that the measured large-scale anisotropies are made of two contributions: the intrinsic inhomogeneities in the temperature at the last scattering surface and the inhomogeneities in the scaling factor provided by the ratio of the frequencies of the photons at the departure and arrival points. Let us first consider the second contribution. As the frequency of the photon is the inverse of a time period, we get immediately the fully nonlinear relation

$$
\frac{\bar{\omega}_{\mathcal{E}}}{\bar{\omega}_{\mathcal{O}}}=\frac{\omega_{\mathcal{E}}}{\omega_{\mathcal{O}}} e^{-\Phi_{\ell \mathcal{E}}+\Phi_{\ell \mathcal{O}}}
$$

As for the temperature anisotropies coming from the intrinsic temperature fluctuation at the emission point, it may be worth to recall how to obtain this quantity in the longitudinal gauge at first order. By expanding the photon energy density $\rho_{\gamma} \propto T_{\gamma}^{4}$, the intrinsic temperature anisotropies at last scattering are given by $\delta_{1} T_{\mathcal{E}} / T_{\mathcal{E}}=$ $(1 / 4) \delta_{1} \rho_{\gamma} / \rho_{\gamma}$. One relates the photon energy density fluctuation to the gravitational perturbation first by implementing the adiabaticity condition $\delta_{1} \rho_{\gamma} / \rho_{\gamma}=(4 / 3) \delta_{1} \rho_{m} / \rho_{m}$, where $\delta_{1} \rho_{m} / \rho_{m}$ is the relative fluctuation in the matter component, and then using the energy constraint of Einstein equations $\phi^{(1)}=-(1 / 2) \delta_{1} \rho_{m} / \rho_{m}$. The result is $\delta_{1} T_{\mathscr{E}} / T_{\mathcal{E}}=-2 \Phi_{1 \mathcal{E}} / 3$. Summing this contribution to the anisotropies coming from the redshift factor (7) expanded at first order provides the standard (linear) Sachs-Wolfe effect $\delta_{1} T_{\mathcal{O}} / T_{\mathcal{O}}=\Phi_{1 \mathcal{E}} / 3$. Following the same steps, we may easily obtain its full nonlinear generalization.

Let us first relate the photon energy density $\bar{\rho}_{\gamma}$ to the energy density of the nonrelativistic matter $\bar{\rho}_{m}$ by using the adiabaticity condition. Again, here a bar indicates that we are considering quantities in the locally homogeneous Universe described by the metric (5). Using the energy continuity equation on large scales $\partial \bar{\rho} / \partial \bar{t}=-3 \bar{H}(\bar{\rho}+\bar{P})$, where $\bar{H}=$ $d \ln \bar{a} / d \bar{t}$ and $\bar{P}$ is the pressure of the fluid, one can easily show that there exists a conserved quantity in time at any order in perturbation theory [53]

$$
\mathcal{F} \equiv \ln \bar{a}+\frac{1}{3} \int^{\bar{\rho}} \frac{d \overline{\rho^{\prime}}}{\left(\overline{\rho^{\prime}}+\overline{P^{\prime}}\right)} .
$$

The perturbation $\delta \mathcal{F}$ is a gauge-invariant quantity representing the nonlinear extension of the curvature perturbation $\zeta$ on uniform energy density hypersurfaces on superhorizon scales for adiabatic fluids [53]. Indeed, expanding 
it at first and second order one gets the corresponding definition $\zeta_{1}=-\psi_{1}-\delta_{1} \rho / \dot{\rho}$ and the quantity $\zeta_{2}$ introduced in [54]. We will come back to these definitions later. At first order, the adiabaticity condition corresponds to set $\zeta_{1 \gamma}=\zeta_{1 m}$ for the curvature perturbations relative to each component. At the nonlinear level the adiabaticity condition generalizes to

$$
\frac{1}{3} \int \frac{d \bar{\rho}_{m}}{\bar{\rho}_{m}}=\frac{1}{4} \int \frac{d \bar{\rho}_{\gamma}}{\bar{\rho}_{\gamma}},
$$

or

$$
\ln \bar{\rho}_{m}=\ln \bar{\rho}_{\gamma}^{3 / 4} .
$$

To make contact with the standard second-order result, we may expand in (10) the photon energy density perturbations as $\delta \bar{\rho}_{\gamma} / \rho_{\gamma}=\delta_{1} \rho_{\gamma} / \rho_{\gamma}+(1 / 2) \delta_{2} \rho_{\gamma} / \rho_{\gamma}$, and similarly for the matter component. We immediately recover the adiabaticity condition

$$
\frac{\delta_{2} \rho_{\gamma}}{\rho_{\gamma}}=\frac{4}{3} \frac{\delta_{2} \rho_{m}}{\rho_{m}}+\frac{4}{9}\left(\frac{\delta_{1} \rho_{m}}{\rho_{m}}\right)^{2},
$$

given in [5].

Next, we need to relate the photon energy density to the gravitational potentials at the nonlinear level. The energy constraint inferred from the $(0-0)$ component of Einstein equations in the matter-dominated era with the "barred" metric (5) is

$$
\bar{H}^{2}=\frac{8 \pi G_{N}}{3} \bar{\rho}_{m} .
$$

Using (4), the Hubble parameter $\bar{H}$ reads

$$
\bar{H}=\frac{1}{\bar{a}} \frac{d \bar{a}}{d \bar{t}}=e^{-\Phi_{\ell}}\left(H-\dot{\Psi}_{\ell}\right),
$$

where $H=d \ln a / d t$ is the Hubble parameter in the "unbarred" metric. Equation (12) thus yields an expression for the energy density of the nonrelativistic matter which is fully nonlinear, being expressed in terms of the gravitational potential $\Phi_{\ell}$

$$
\bar{\rho}_{m}=\rho_{m} e^{-2 \Phi_{\ell}},
$$

where we have dropped $\dot{\Psi}_{\ell}$ which is negligible on large scales. By perturbing, (14) we are able to recover in a straightforward way the solutions of the $(0-0)$ component of Einstein equations for a matter-dominated Universe in the large-scale limit obtained at second order in perturbation theory. Indeed, recalling that $\Phi$ is perturbatively related to the quantity $\phi=\phi^{(1)}+\phi^{(2)} / 2$ used in [5] by $\phi^{(1)}=\phi^{(1)}$ and $\phi^{(2)}=\phi^{(2)}-2\left(\phi^{(1)}\right)^{2}$, one immediately obtains

$$
\begin{aligned}
\frac{\delta_{1} \rho_{m}}{\rho_{m}} & =-2 \phi^{(1)}, \\
\frac{1}{2} \frac{\delta_{2} \rho_{m}}{\rho_{m}} & =-\phi^{(2)}+4\left(\phi^{(1)}\right)^{2} .
\end{aligned}
$$

The expression for the intrinsic temperature of the photons at the last scattering surface $\bar{T}_{\mathcal{E}} \propto \bar{\rho}_{\gamma}^{1 / 4}$ follows from (10) and (14)

$$
\bar{T}_{\mathcal{E}}=T_{\mathcal{E}} e^{-2 \Phi_{\ell} / 3} .
$$

Plugging (7) and (16) into (6), we are finally able to provide the expression for the $\mathrm{CMB}$ temperature which is fully nonlinear and takes into account both the gravitational redshift of the photons due to the metric perturbations at last scattering and the intrinsic temperature anisotropies

$$
\bar{T}_{\mathcal{O}}=\left(\frac{\omega_{\mathcal{O}}}{\omega_{\mathcal{E}}}\right) T_{\mathcal{E}} e^{\Phi_{\ell} / 3} .
$$

From (17), we read the nonperturbative anisotropy corresponding to the Sachs-Wolfe effect

$$
\frac{\delta_{n p} \bar{T}_{\mathcal{O}}}{T_{\mathcal{O}}}=e^{\Phi_{\ell} / 3}-1
$$

Equation (18) is one of the main results of this paper and represents at any order in perturbation theory the extension of the linear Sachs-Wolfe effect. At first order, one gets

$$
\frac{\delta_{1} T_{\mathcal{O}}}{T_{\mathcal{O}}}=\frac{1}{3} \phi^{(1)}
$$

and at second order

$$
\frac{1}{2} \frac{\delta_{2} T_{\mathcal{O}}}{T_{\mathcal{O}}}=\frac{1}{6} \phi^{(2)}+\frac{1}{18}\left(\phi^{(1)}\right)^{2} .
$$

This result shows that the $\mathrm{CMB}$ anisotropies is nonlinear on large scales and that a source of NG is inevitably sourced by gravity.

\section{Perturbing Gravity}

In this Section, we provide the necessary tools to deal with perturbed gravity, giving the expressions for the Einstein tensor perturbed up to second-order around a flat FriedmannRobertson-Walker background, and the relevant Einstein equations. In the following, we will adopt the Poisson gauge which eliminates one scalar degree of freedom from the $g_{0 i}$ component of the metric and one scalar and two vector degrees of freedom from $g_{i j}$. We rewrite the metric (1) as

$$
d s^{2}=a^{2}(\eta)\left[-e^{2 \Phi} d \eta^{2}+2 \omega_{i} d x^{i} d \eta+\left(e^{-2 \Psi} \delta_{i j}+\chi_{i j}\right) d x^{i} d x^{j}\right],
$$

where $a(\eta)$ is the scale factor as a function of the conformal time $\eta$. As we previously mentioned, for the vector and tensor perturbations, we will neglect linear vector modes since they are not produced in standard mechanisms for the generation of cosmological perturbations (as inflation), and we also neglect tensor modes at linear order, since they give a negligible contribution to second-order perturbations. Therefore, we take $\omega_{i}$ and $\chi_{i j}$ to be second-order vector and tensor perturbations of the metric. 
Let us now give our definitions for the connection coefficients and their expressions for the metric (1). The number of spatial dimensions is $n=3$. Greek indices $(\alpha, \beta, \ldots, \mu, \nu, \ldots)$ run from 0 to 3 , while Latin indices $(a, b, \ldots, i, j, k, \ldots m, n \ldots)$ run from 1 to 3 . The total spacetime metric $g_{\mu \nu}$ has signature $(-,+,+,+)$. The connection coefficients are defined as

$$
\Gamma_{\beta \gamma}^{\alpha}=\frac{1}{2} g^{\alpha \rho}\left(\frac{\partial g_{\rho \gamma}}{\partial x^{\beta}}+\frac{\partial g_{\beta \rho}}{\partial x^{\gamma}}-\frac{\partial g_{\beta \gamma}}{\partial x^{\rho}}\right) .
$$

The Riemann tensor is defined as

$$
R_{\beta \mu \nu}^{\alpha}=\Gamma_{\beta \nu, \mu}^{\alpha}-\Gamma_{\beta \mu, \nu}^{\alpha}+\Gamma_{\lambda \mu}^{\alpha} \Gamma_{\beta \nu}^{\lambda}-\Gamma_{\lambda \nu}^{\alpha} \Gamma_{\beta \mu}^{\lambda}
$$

The Ricci tensor is a contraction of the Riemann tensor

$$
R_{\mu \nu}=R_{\mu \alpha \gamma}^{\alpha}
$$

and in terms of the connection coefficient it is given by

$$
R_{\mu \nu}=\partial_{\alpha} \Gamma_{\mu \nu}^{\alpha}-\partial_{\mu} \Gamma_{\nu \alpha}^{\alpha}+\Gamma_{\sigma \alpha}^{\alpha} \Gamma_{\mu \nu}^{\sigma}-\Gamma_{\sigma \nu}^{\alpha} \Gamma_{\mu \alpha}^{\sigma} .
$$

The Ricci scalar is given by contracting the Ricci tensor

$$
R=R_{\mu}^{\mu}
$$

The Einstein tensor is defined as

$$
G_{\mu \nu}=R_{\mu \nu}-\frac{1}{2} g_{\mu \nu} R
$$

3.1. The Connection Coefficients. For the connection coefficients we find

$$
\begin{aligned}
\Gamma_{00}^{0}= & \mathscr{H}+\Phi^{\prime}, \\
\Gamma_{0 i}^{0}= & \frac{\partial \Phi}{\partial x^{i}}+\mathscr{H} \omega_{i}, \\
\Gamma_{00}^{i}= & \omega^{i^{\prime}}+\mathscr{H} \omega^{i}+e^{2 \Psi+2 \Phi} \frac{\partial \Phi}{\partial x_{i}}, \\
\Gamma_{i j}^{0}= & -\frac{1}{2}\left(\frac{\partial \omega_{j}}{\partial x^{i}}+\frac{\partial \omega_{i}}{\partial x^{j}}\right)+e^{-2 \Psi-2 \Phi}\left(\mathcal{H}-\Psi^{\prime}\right) \delta_{i j} \\
& +\frac{1}{2} \chi_{i j}^{\prime}+\mathscr{H} \chi_{i j}, \\
\Gamma_{0 j}^{i}= & \left(\mathcal{H}-\Psi^{\prime}\right) \delta_{i j}+\frac{1}{2} \chi_{i j}^{\prime}+\frac{1}{2}\left(\frac{\partial \omega_{i}}{\partial x^{j}}-\frac{\partial \omega_{j}}{\partial x^{i}}\right), \\
\Gamma_{j k}^{i}= & -\mathscr{H} \omega^{i} \delta_{j k}-\frac{\partial \Psi}{\partial x^{k}} \delta_{j}^{i}-\frac{\partial \Psi}{\partial x^{j}} \delta_{k}^{i}+\frac{\partial \Psi}{\partial x_{i}} \delta_{j k} \\
& +\frac{1}{2}\left(\frac{\partial \chi_{j}^{i}}{\partial x^{k}}+\frac{\partial \chi_{k}^{i}}{\partial x^{j}}+\frac{\partial \chi_{j k}}{\partial x_{i}}\right) .
\end{aligned}
$$

3.2. The Einstein Equations. The Einstein equations are written as $G_{\mu \nu}=\kappa^{2} T_{\mu \nu}$, so that $\kappa^{2}=8 \pi G_{\mathrm{N}}$, where $G_{\mathrm{N}}$ is the usual Newtonian gravitational constant. They read

$$
\begin{aligned}
G_{0}^{0}= & -\frac{e^{-2 \Phi}}{a^{2}}\left[3 \mathcal{H}^{2}-6 \mathcal{H} \Psi^{\prime}+3\left(\Psi^{\prime}\right)^{2}\right. \\
& \left.-e^{2 \Phi+2 \Psi}\left(\partial_{i} \Psi \partial^{i} \Psi-2 \nabla^{2} \Psi\right)\right]=\kappa^{2} T_{0}^{0}, \\
G_{0}^{i}= & 2 \frac{e^{2 \Psi}}{a^{2}}\left[\partial^{i} \Psi^{\prime}+\left(\mathcal{H}-\Psi^{\prime}\right) \partial^{i} \Phi\right] \\
& -\frac{1}{2 a^{2}} \nabla^{2} \omega^{i}+\left(4 \mathcal{H}^{2}-2 \frac{a^{\prime \prime}}{a}\right) \frac{\omega^{i}}{a^{2}}=\kappa^{2} T_{0}^{i}, \\
G_{j}^{i}= & \frac{1}{a^{2}}\left[e ^ { - 2 \Phi } \left(\mathcal{H}^{2}-2 \frac{a^{\prime \prime}}{a}-2 \Psi^{\prime} \Phi^{\prime}\right.\right. \\
& \left.-3\left(\Psi^{\prime}\right)^{2}+2 \mathscr{H}\left(\Phi^{\prime}+2 \Psi^{\prime}\right)+2 \Psi^{\prime \prime}\right) \\
& +\frac{\left.e^{2 \Psi}\left(\partial_{k} \Phi \partial^{k} \Phi+\nabla^{2} \Phi-\nabla^{2} \Psi\right)\right] \delta_{j}^{i}}{a^{2}}\left(-\partial^{i} \Phi \partial_{j} \Phi-\partial^{i} \partial_{j} \Phi+\partial^{i} \partial_{j} \Psi\right. \\
& \left.-\partial^{i} \Phi \partial_{j} \Psi+\partial^{i} \Psi \partial_{j} \Psi-\partial^{i} \Psi \partial_{j} \Phi\right) \\
& -\frac{\mathscr{H}}{a^{2}}\left(\partial^{i} \omega_{j}+\partial_{j} \omega^{i}\right)-\frac{1}{2 a^{2}}\left(\partial^{i} \omega_{j^{\prime}}+\partial_{j} \omega^{i^{\prime}}\right) \\
& +\frac{1}{a^{2}}\left(\mathscr{H} \chi_{j}^{i^{\prime}}+\frac{1}{2} \chi_{j}^{i^{\prime \prime}}-\frac{1}{2} \nabla^{2} \chi_{j}^{i}\right)=\kappa^{2} T_{j}^{i} .
\end{aligned}
$$

Taking the traceless part of (31), we find

$$
\Psi-\Phi=\mathcal{Q},
$$

where $\mathcal{Q}$ is defined by $\nabla^{2} \mathcal{Q}=-P+3 N$, with $P \equiv P^{i}{ }_{i}$,

$$
P_{j}^{i}=\partial^{i} \Phi \partial_{j} \Psi+\frac{1}{2}\left(\partial^{i} \Phi \partial_{j} \Phi-\partial^{i} \Psi \partial_{j} \Psi\right)+4 \pi G_{\mathrm{N}} a^{2} e^{-2 \Psi} T_{j}^{i},
$$

and $\nabla^{2} N=\partial_{i} \partial^{j} P_{j}^{i}$. The trace of (31) gives

$$
\begin{aligned}
& e^{-2 \Phi}\left(\mathcal{H}^{2}-2 \frac{a^{\prime \prime}}{a}-2 \Phi^{\prime} \Psi^{\prime}\right. \\
& \left.-3\left(\Psi^{\prime}\right)^{2}+2 \mathcal{H}\left(3 \Psi^{\prime}-\mathcal{Q}^{\prime}\right)+2 \Psi^{\prime \prime}\right) \\
& +\frac{e^{2 \Psi}}{3}\left(2 \partial_{k} \Phi \partial^{k} \Phi+\partial_{k} \Psi \partial^{k} \Psi-2 \partial_{k} \Phi \partial^{k} \Psi+2(P-3 N)\right) \\
& =\frac{8 \pi G_{N}}{3} a^{2} T_{k}^{k} .
\end{aligned}
$$


From (30), we may deduce an equation for $\omega^{i}$

$$
\begin{aligned}
- & \frac{1}{2} \nabla^{2} \omega^{i}+\left(4 \mathscr{H}^{2}-2 \frac{a^{\prime \prime}}{a}\right) \omega^{i} \\
= & -\left(\delta_{j}^{i}-\frac{\partial^{i} \partial_{j}}{\nabla^{2}}\right) \\
& \times\left(2\left(\partial^{j} \Psi^{\prime}+\left(\mathscr{H}-\Psi^{\prime}\right) \partial^{j} \Phi\right)-8 \pi G_{\mathrm{N}} a^{2} e^{-2 \Psi} T_{0}^{j}\right) .
\end{aligned}
$$

Here, $T_{\nu}^{\mu}$ is the energy momentum tensor accounting for different components, photons, baryons, and dark matter. We will give the expressions later for each component in terms of the distribution functions.

\section{Setting the Initial Conditions from the Primordial NG}

In this paper we are concerned with the second-order evolution of the cosmological perturbations. This requires that we well define the initial conditions of the cosmological perturbations at second order. These boundary conditions may or may not contain already some level of NG. It they do, we say that there exist some primordial NG. The latter is usually defined in the epoch in which the comoving curvature perturbation remains constant on large superhorizon scales. In the standard single-field inflationary model, the first seeds of density fluctuations are generated on superhorizon scales from the fluctuations of a scalar field, the inflaton [1].

In order to follow the evolution on superhorizon scales of the density fluctuations coming from the various mechanisms, we use the curvature perturbation of uniform density hypersurfaces $\zeta=\zeta^{(1)}+\zeta^{(2)} / 2+\cdots$, where $\zeta^{(1)}=-\psi^{(1)}-$ $\mathscr{H} \delta \rho^{(1)} / \bar{\rho}^{\prime}$ and the expression for $\zeta^{(2)}$ is given by [54]

$$
\zeta^{(2)}=-\psi^{(2)}-\mathscr{H} \frac{\delta^{(2)} \rho}{\rho^{\prime}}+\Delta \zeta^{(2)},
$$

with

$$
\begin{aligned}
\Delta \zeta^{(2)}= & 2 \mathscr{H} \frac{\delta^{(1)} \rho^{\prime}}{\rho^{\prime}} \frac{\delta^{(1)} \rho}{\rho^{\prime}}+2 \frac{\delta^{(1)} \rho}{\rho^{\prime}}\left(\psi^{(1)^{\prime}}+2 \mathscr{H} \psi^{(1)}\right) \\
& -\left(\frac{\delta^{(1)} \rho}{\rho^{\prime}}\right)^{2}\left(\mathscr{H} \frac{\rho^{\prime \prime}}{\rho}-\mathscr{H}^{\prime}-2 \mathscr{H}^{2}\right)+2 \psi^{(1) 2}
\end{aligned}
$$

The crucial point is that the gauge-invariant curvature perturbation $\zeta$ remains constant on superhorizon scales after it has been generated during a primordial epoch and possible isocurvature perturbations are no longer present. Therefore, we may set the initial conditions at the time when $\zeta$ becomes constant. In particular, $\zeta^{(2)}$ provides the necessary information about the "primordial" level of non-Gaussianity generated either during inflation, as in the standard scenario, or immediately after it, as in the curvaton scenario. Different scenarios are characterized by different values of $\zeta^{(2)}$. For example, in the standard single-field inflationary model $\zeta^{(2)}=2\left(\zeta^{(1)}\right)^{2}+\mathcal{O}(\epsilon, \eta)[6,7]$, where $\epsilon$ and $\eta$ are the standard slow-roll parameters [1]. In general, we may parametrize the primordial non-Gaussianity level in terms of the conserved curvature perturbation as in [34]

$$
\zeta^{(2)}=2 a_{\mathrm{NL}}\left(\zeta^{(1)}\right)^{2}
$$

where the parameter $a_{\mathrm{NL}}$ depends on the physics of a given scenario. For example in the standard scenario $a_{\mathrm{NL}} \simeq 1$, while in the curvaton case $a_{\mathrm{NL}}=(3 / 4 r)-r / 2$, where $r \approx\left(\rho_{\sigma} / \rho\right)_{\mathrm{D}}$ is the relative curvaton contribution to the total energy density at curvaton decay $[5,55]$. Alternatives to the curvaton model are those models characterized by the curvature perturbation being generated by an inhomogeneity in the decay rate [1113] or the mass [14] or of the particles responsible for the reheating after inflation. Other opportunities for generating the curvature perturbation occur at the end of inflation [16-18] and during preheating [19-21]. All these models generate a level of NG which is local as the NG part of the primordial curvature perturbation is a local function of the Gaussian part, being generated on superhorizon scales. In momentum space, the three point function, or bispectrum, arising from the local NG is dominated by the so-called "squeezed" configuration, where one of the momenta is much smaller than the other two. Other models, such as DBI inflation [23] and ghost inflation [56], predict a different kind of primordial NG, called "equilateral", because the three-point function for this kind of NG is peaked on equilateral configurations, in which the length of the three wavevectors forming a triangle in Fourier space are equal [25].

One of the best tools to detect or constrain the primordial large-scale non-Gaussianity is through the analysis of the $\mathrm{CMB}$ anisotropies, for example by studying the bispectrum [5]. In that case, the standard procedure is to introduce the primordial nonlinearity parameter $f_{\mathrm{NL}}$ characterizing the primordial non-Gaussianity via the curvature perturbation [5] (the sign convention is such that it follows the WMAP convention for $f_{\mathrm{NL}}$, see Section 5)

$$
\zeta=\zeta^{(1)}+\frac{3}{5} f_{\mathrm{NL}}\left(\zeta^{(1)} \star \zeta^{(1)}\right)
$$

where the coefficient $3 / 5$ arises from the first-order relation connecting the comoving curvature perturbation and the gravitational potential, $\zeta^{(1)}=-5 \phi^{(1)} / 3$, and the $\star$-product reminds us that one has to perform a convolution product in momentum space and that $f_{\mathrm{NL}}$ is indeed momentumdependent (we refer the reader to the end of Section 5 for a detailed discussion about the definition of the nonlinearity parameter). To give the feeling of the resulting size of $f_{\mathrm{NL}}$ when $\left|a_{\mathrm{NL}}\right| \gg 1, f_{\mathrm{NL}} \simeq 5 a_{\mathrm{NL}} / 3$ (see $[5,34]$ ). Present limits on NG are summarized by $-4<f_{\mathrm{NL}}^{\text {loc }}<80$ [57] and $-125<$ $f_{\mathrm{NL}}^{\text {equil }}<435$ [58] at 95\% CL, where $f_{\mathrm{NL}}^{\text {loc }}$ and $f_{\mathrm{NL}}^{\text {equil }}$ stand for the nonlinear parameter in the case in which the squeezed and the equilateral configurations dominate, respectively. 


\section{CMB Anisotropies at Second-Order on Large Scales}

In this section, we provide the exact expression for large-scale $\mathrm{CMB}$ temperature fluctuations at second order in perturbation theory. What this section contains, therefore, should be considered as a more technical elaboration of what the reader can find in Section 2. The final expression, we will find has various virtues. First, from it one can unambiguously extract the exact definition of the nonlinearity parameter $f_{\mathrm{NL}}$ which is used by the experimental collaborations to pin down the level of NG in the temperature fluctuations. Second, it contains a "primordial" term encoding all the information about the NG generated in primordial epochs, namely during or immediately after inflation, and depends upon the various fluctuation generation mechanisms. As such, the expression neatly disentangles the primordial contribution to the NG from that arising after inflation. Finally, the expression applies to all scenarios for the generation of cosmological perturbations. Third, it is gauge-invariant. For this last point, let us underline however that the gaugeinvariant expression that we will provide is just one of the many possible ways in which the $\mathrm{CMB}$ temperature fluctuations can be casted in a gauge-invariant form.

In order to obtain our gauge-independent formula for the temperature anisotropies, we again perturb the spatially flat Robertson-Walker background. We expand the metric perturbations (21) in a first and a second-order part as

$$
\begin{aligned}
g_{00} & =-a^{2}\left(1+2 \phi^{(1)}+\phi^{(2)}+2\left(\phi^{(1)}\right)^{2}\right) \\
g_{0 i} & =a^{2}\left(\omega_{i}^{(1)}+\frac{1}{2} \omega_{i}^{(2)}\right) \\
g_{i j} & =a^{2}\left[\left(1-2 \psi^{(1)}-\psi^{(2)}+2\left(\psi^{(1)}\right)^{2}\right) \delta_{i j}+\left(\chi_{i j}^{(1)}+\frac{1}{2} \chi_{i j}^{(2)}\right)\right] .
\end{aligned}
$$

Again, the functions $\phi^{(r)}, \omega_{i}^{(r)}, \psi^{(r)}$ and $\chi_{i j}^{(r)}$, where $(r)=$ $(1,2)$, stand for the $r$ th-order perturbations of the metric. We can split $\omega_{i}^{(r)}=\partial_{i} \omega^{(r)}+\omega_{i}^{(r)}$, where $\omega^{(r)}$ is the scalar part and $\omega_{i}^{(r)}$ is a transverse vector, that is, $\partial^{i} \omega_{i}^{(r)}=0$. The metric perturbations will transform according to an infinitesimal change of coordinates. From now on, we limit ourselves to a second-order time shift [51]

$$
\eta \longrightarrow \eta-\alpha_{(1)}+\frac{1}{2}\left(\alpha_{(1)}^{\prime} \alpha_{(1)}-\alpha_{(2)}\right)
$$

where a prime denotes differentiation w.r.t. conformal time. In general, a gauge corresponds to a choice of coordinates defining a slicing of spacetime into hypersurfaces (at fixed time $\eta$ ) and a threading into lines (corresponding to fixed spatial coordinates $x$ ), but in this section only the former is relevant so that gauge-invariant can be taken to mean independent of the slicing. For example, under the time shift, the first-order spatial curvature perturbation $\psi^{(1)}$ transforms as $\psi^{(1)} \rightarrow \psi^{(1)}-\mathscr{H} \alpha_{(1)}$ (here $\left.\mathscr{H}=a^{\prime} / a\right)$, while $\phi^{(1)} \rightarrow$ $\phi^{(1)}+\alpha_{(1)}^{\prime}+\mathcal{H} \alpha^{(1)}, \omega_{i}^{(1)} \rightarrow \omega_{i}^{(1)}-\partial_{i} \alpha^{(1)}$, and the traceless part of the spatial metric $\chi_{i j}^{(1)}$ turns out to be gauge-invariant. At second order in the perturbations, we just give some useful examples like the transformation of the energy density and the curvature perturbation [51]. (For these two examples, we here report only the expression of the second-order gauge transformations neglecting gradient terms on large-scales. Notice that the expressions of this section relative to linear perturbations are valid on all scales, while for the secondorder fluctuations at some point the large-scale limit is considered.)

$$
\begin{aligned}
\delta^{(2)} \rho \longrightarrow & \delta^{(2)} \rho+\rho^{\prime} \alpha_{(2)}+\alpha_{(1)}\left(\rho^{\prime \prime} \alpha_{(1)}+\rho^{\prime} \alpha_{(1)}^{\prime}+2 \delta^{(1)} \rho^{\prime}\right) \\
\psi^{(2)} \longrightarrow & \psi^{(2)}+2 \alpha_{(1)} \psi^{(1)^{\prime}}-\mathscr{H} \alpha_{(1)}^{2}-\mathscr{H} \alpha_{(1)} \alpha_{(1)}^{\prime} \\
& -\frac{1}{3}\left(2 \omega_{(1)}^{i}-\alpha_{(1)}^{i}\right) \alpha_{, i}^{(1)}-\mathscr{H} \alpha_{(2)} .
\end{aligned}
$$

We now construct in a gauge-invariant way temperature anisotropies at second order. Temperature anisotropies beyond the linear regime have been calculated in [32, 51], following the photons path from last scattering to the observer in terms of perturbed geodesics. The linear temperature anisotropies read $[32,51]$

$$
\frac{\Delta T^{(1)}}{T}=\phi_{\mathcal{E}}^{(1)}-v_{\mathcal{E}}^{(1) i} e_{i}+\tau_{\mathcal{E}}^{(1)}-\int_{\lambda_{\mathscr{G}}}^{\lambda_{\mathcal{E}}} d \lambda A^{(1)^{\prime}},
$$

where $A^{(1)} \equiv \psi^{(1)}+\phi^{(1)}+\omega_{i}^{(1)} e^{i}-(1 / 2) \chi_{i j}^{(1)} e^{i} e^{j}$, the subscript $\&$ indicates that quantities are evaluated at last-scattering, $e^{i}$ is a spatial unit vector specifying the direction of observation and the integral is evaluated along the line-of-sight parametrized by the affine parameter $\lambda$. Equation (43) includes the intrinsic fractional temperature fluctuation at emission $\tau_{\mathcal{E}}$, the Doppler effect due to emitter's velocity $v_{\mathcal{E}}^{(1) i}$ and the gravitational redshift of photons, including the integrated Sachs-Wolfe (ISW) effect. We omitted monopoles due to the observer $\mathcal{O}$ (e.g., the gravitational potential $\psi_{\mathcal{O}}^{(1)}$ evaluated at the event of observation), which, being independent of the angular coordinate, can be always recast into the definition of temperature anisotropies. Notice, however, that the physical meaning of each contribution in (43) is not gauge-invariant, as the different terms are gauge-dependent. However, it is easy to show that the whole expression (43) is gauge-invariant. Since the temperature $T$ is a scalar, the intrinsic temperature fluctuation transforms as $\tau_{\mathcal{E}}^{(1)} \rightarrow$ $\tau_{\mathcal{E}}^{(1)}+\left(T^{\prime} / T\right) \alpha_{(1)}=\tau_{\mathcal{E}}^{(1)}-\mathcal{H} \alpha_{(1)}$, having used the fact that the temperature scales as $T \propto a^{-1}$. Notice, instead, that the velocity $v_{\mathcal{E}}^{(1) i}$ does not change. Therefore, using the transformations of metric perturbations we find

$$
\begin{aligned}
\frac{\Delta T^{(1)}}{T} & \longrightarrow \frac{\Delta T^{(1)}}{T}+\alpha_{(1)}^{\prime}-\int_{\eta_{\mathcal{O}}}^{\eta_{\mathcal{E}}} d \eta \frac{d \alpha_{(1)}^{\prime}}{d \eta} \\
& =\frac{\Delta T^{(1)}}{T}+\mathcal{O},
\end{aligned}
$$

where we have used the fact that the integral is evaluated along the line-of-sight which can be parametrized by the 
background geodesics $x^{(0) \mu}=\left(\lambda,\left(\lambda_{\mathcal{O}}-\lambda_{\varepsilon}\right) e^{i}\right)$ (with $d \lambda / d \eta=$ 1 ), and the decomposition for the total derivative along the path for a generic function $f\left(\lambda, x^{i}(\lambda)\right), f^{\prime}=\partial f / \partial \lambda=d f / d \lambda+$ $\partial_{i} f e^{i}$. Equation (44) shows that the expression (43) for firstorder temperature anisotropies is indeed gauge-invariant (up to monopole terms related to the observer $\mathcal{O}$ ). Temperature anisotropies can be easily written in terms of particular combinations of perturbations which are manifestly gaugeinvariant. For the gravitational potentials, we consider the gauge-invariant definitions $\psi_{\mathrm{GI}}^{(1)}=\psi^{(1)}-\mathcal{H} \omega^{(1)}$ and $\phi_{\mathrm{GI}}^{(1)}=$ $\phi^{(1)}+\mathcal{H} \omega^{(1)}+\omega^{(1)^{\prime}}$. For the $(0-i)$ component of the metric and the traceless part of the spatial metric, we define $\omega_{i}^{(1) \mathrm{GI}}=\omega_{i}^{(1)}$ and $\chi_{i j}^{(1) \mathrm{GI}}=\chi_{i j}^{(1)}$. For the matter variables we use a gauge-invariant intrinsic temperature fluctuation $\tau_{\mathrm{GI}}^{(1)}=\tau^{(1)}-\mathcal{H} \omega^{(1)}$, while the velocity itself is gauge-invariant $v_{\mathrm{GI}}^{(1) i}=v^{(1) i}$ under time shifts. Following the same steps leading to (44), one gets the linear temperature anisotropies in (43) in terms of these gauge-invariant quantities

$$
\frac{\Delta T_{\mathrm{GI}}^{(1)}}{T}=\phi_{\mathrm{GI}}^{(1)}-v_{\mathrm{GI}}^{(1) i} e_{i}+\tau_{\mathrm{GI}}^{(1)}-\int_{\lambda_{\Theta}}^{\lambda_{\varepsilon}} d \lambda A_{\mathrm{GI}}^{(1)^{\prime}},
$$

where $A_{\mathrm{GI}}^{(1)}=\phi_{\mathrm{GI}}^{(1)}+\psi_{\mathrm{GI}}^{(1)}+\omega_{i}^{(1) \mathrm{GI}} e_{i}-(1 / 2) \chi_{i j}^{(1) \mathrm{GI}} e^{i} e^{j}$ and we omitted the subscript $\mathcal{E}$. For the primordial fluctuations we are interested in the large-scale modes set by the curvature perturbation $\zeta^{(1)}$. Defining a gauge-invariant density perturbation $\delta^{(1)} \rho_{\mathrm{GI}}=\delta^{(1)} \rho+\rho^{\prime} \omega^{(1)}$, we write the curvature perturbation as $\zeta_{\mathrm{GI}}^{(1)}=-\psi_{\mathrm{GI}}^{(1)}-\mathcal{H}\left(\delta^{(1)} \rho_{\mathrm{GI}} / \rho^{\prime}\right)$. Since for adiabatic perturbations in the radiation $(\gamma)$ and matter $(m)$ eras $(1 / 4)\left(\delta^{(1)} \rho_{\gamma} / \rho_{\gamma}\right)=(1 / 3)\left(\delta^{(1)} \rho_{m} / \rho_{m}\right)$, one can write the intrinsic temperature fluctuation as $\tau^{(1)}=$ $(1 / 4)\left(\delta^{(1)} \rho_{\gamma} / \rho_{\gamma}\right)=-\mathscr{H}\left(\delta^{(1)} \rho / \rho^{\prime}\right)$ and a gauge-invariant definition is $\tau_{\mathrm{GI}}^{(1)}=-\mathscr{H}\left(\delta^{(1)} \rho_{\mathrm{GI}} / \rho^{\prime}\right)$. In the large-scale limit, from Einstein equations $(i-i)$ and continuity equations, in the matter era $\phi_{\mathrm{GI}}^{(1)}=\psi_{\mathrm{GI}}^{(1)}=-(3 / 5) \zeta_{\mathrm{GI}}^{(1)}$. Thus we obtain the large-scale limit of temperature anisotropies (45) $\Delta T_{\mathrm{GI}}^{(1)} / T=$ $2 \psi_{\mathrm{GI}}^{(1)}+\zeta_{\mathrm{GI}}^{(1)}=\psi_{\mathrm{GI}}^{(1)} / 3$, that is, the usual Sachs-Wolfe effect.

At second order, the procedure is similar to the one described so long, though more lengthy and cumbersome. We only provide the reader with the main steps to get the final expression. The second-order temperature fluctuations in terms of metric perturbations read $[32,59]$

$$
\begin{aligned}
\frac{\Delta T^{(2)}}{T}= & \frac{1}{2} \phi_{\mathcal{E}}^{(2)}-\frac{1}{2}\left(\phi^{(1)}\right)^{2}-\frac{1}{2} v_{\mathcal{E}}^{(2) i} e_{i}+\frac{1}{2} \tau_{\mathcal{E}}^{(2)}-I_{2}\left(\lambda_{\mathcal{E}}\right) \\
& +\left(I_{1}\left(\lambda_{\mathcal{E}}\right)+v_{\mathcal{E}}^{(1) i} e_{i}\right)\left(-\phi_{\mathcal{E}}^{(1)}-\tau_{\mathcal{E}}^{(1)}+v_{\mathcal{E}}^{(1) i} e_{i}+I_{1}\left(\lambda_{\mathcal{E}}\right)\right) \\
& +x_{\mathcal{E}}^{(1) 0} A_{\mathcal{E}}^{(1)^{\prime}}+\left(x_{\mathcal{E}}^{(1) j}+x_{\mathcal{E}}^{(1) 0} e^{j}\right)\left(\phi_{, j}^{(1)}-v_{i, j}^{(1)} e^{i}+\tau_{, j}^{(1)}\right)_{\mathcal{E}} \\
& -\frac{1}{2} v_{\mathcal{E} i}^{(1)} v_{\mathcal{E}}^{(1) i}+\phi_{\mathcal{E}}^{(1)} \tau_{\mathcal{E}}^{(1)}+\frac{\partial \tau^{(1)}}{\partial d^{i}} d^{(1) i}-v_{\mathcal{E}}^{(1) i} e_{i} \phi_{\mathcal{E}}^{(1)} \\
& +v_{\mathcal{E} i}^{(1)}\left(-\omega_{\mathcal{E}}^{(1) i}-I_{1}^{i}\left(\lambda_{\mathcal{E}}\right)\right) .
\end{aligned}
$$

Here, $I_{2}$ is the second-order ISW [59]

$$
\begin{gathered}
I_{2}\left(\lambda_{\mathcal{E}}\right) \\
=\int_{\lambda_{\mathcal{\Theta}}}^{\lambda_{\mathcal{E}}} d \lambda\left[\frac{1}{2} A^{(2)^{\prime}}-\left(\omega_{i}^{(1)^{\prime}}-\chi_{i j}^{(1)^{\prime}} e^{j}\right)\left(k^{(1) i}+e^{i} k^{(1) 0}\right)\right. \\
+2 k^{(1) 0} A^{(1)^{\prime}}+2 \psi^{(1)^{\prime}} A^{(1)} \\
\left.+x^{(1) 0} A^{(1)^{\prime}}+x^{(1) i} A_{, i}^{(1)^{\prime}}\right],
\end{gathered}
$$

where $A^{(2)} \equiv \psi^{(2)}+\phi^{(2)}+\omega_{i}^{(2)} e^{i}-(1 / 2) \chi_{i j}^{(2)} e^{i} e^{j}$, while $k^{(1) 0}(\lambda)=$ $-2 \phi^{(1)}-\omega^{(1) i} e_{i}+I_{1}(\lambda)$ and $k^{(1) i}(\lambda)=-2 \psi^{(1)} e^{i}-\omega^{(1) i}+\chi^{(1) i j} e_{j}-$ $I_{1}^{i}(\lambda)$ are the photon wave vectors, with $I_{1}(\lambda)$ given by the integral in (43) and $I_{1}^{i}(\lambda)$ is obtained from the same integral replacing the time derivative with a spatial gradient. Finally, in (46)

$$
\begin{aligned}
& x^{(1) 0}(\lambda)=\int_{\lambda_{\Theta}}^{\lambda} d \lambda^{\prime}\left[-2 \phi^{(1)}-\omega_{i}^{(1)} e^{i}+\left(\lambda-\lambda^{\prime}\right) A^{(1)^{\prime}}\right], \\
& x^{(1) i}(\lambda)=-\int_{\lambda_{\Theta}}^{\lambda} d \lambda^{\prime}\left[2 \psi^{(1)} e^{i}+\omega^{(1) i}-\chi^{(1) i j} e_{j}+\left(\lambda-\lambda^{\prime}\right) A^{(1), i}\right],
\end{aligned}
$$

are the geodesics at first order, and $d^{(1) i}=e^{i}-\left(e^{i}-k^{(1) i}\right) / \mid e^{i}-$ $k^{(1) i} \mid$ is the direction of the photon emission evaluated on the hypersurface of constant time of emission $\eta=\eta_{\varepsilon}$. As usual we have omitted the monopole terms due to the observer. Using the transformation rules of [51], it is possible to check that the expression (46) is gauge-invariant. We can express the second-order anisotropies in terms of explicitly gaugeinvariant quantities, whose definition proceeds as for the linear case, by choosing the shifts $\alpha^{(r)}$ such that $\omega^{(r)}=0$. For example, we consider the gauge-invariant gravitational potential [33]

$$
\begin{aligned}
\phi_{\mathrm{GI}}^{(2)}=\phi^{(2)}+\omega^{(1)}\left[2\left(\psi^{(1)^{\prime}}+2 \frac{a^{\prime}}{a} \psi^{(1)}\right)\right. & \\
& \left.+\omega^{(1)^{\prime \prime}}+5 \frac{a^{\prime}}{a} \omega^{(1)^{\prime}}+\left(\mathcal{H}^{\prime}+2 \mathcal{H}^{2}\right) \omega^{(1)}\right] \\
+ & 2 \omega^{(1)^{\prime}}\left(2 \psi^{(1)}+\omega^{(1)^{\prime}}\right)+\frac{1}{a}\left(a \alpha^{(2)}\right),
\end{aligned}
$$

where $\alpha^{(2)}=\omega^{(2)}+\omega^{(1)} \omega^{(1)^{\prime}}+\nabla^{-2} \partial^{i}\left[-4 \psi^{(1)} \partial_{i} \omega^{(1)}-\right.$ $\left.2 \omega^{(1)^{\prime}} \partial_{i} \omega^{(1)}\right]$. Expressing the second-order temperature anisotropies (46) in terms of our gauge-invariant quantities and taking the large-scale limit, we find

$$
\frac{\Delta T_{\mathrm{GI}}^{(2)}}{T}=\left(\frac{1}{2}\right) \phi_{\mathrm{GI}}^{(2)}-\left(\frac{1}{2}\right)\left(\phi_{\mathrm{GI}}^{(1)}\right)^{2}+\left(\frac{1}{2}\right) \tau_{\mathrm{GI}}^{(2)}+\phi_{\mathrm{GI}}^{(1)} \tau_{\mathrm{GI}}^{(1)},
$$

(having dropped the subscript $\mathcal{E}$ ), and the gauge-invariant intrinsic temperature fluctuation at emission is

$$
\tau_{\mathrm{GI}}^{(2)}=\left(\frac{1}{4}\right)\left(\frac{\delta^{(2)} \rho_{\gamma}^{\mathrm{GI}}}{\rho_{\gamma}}\right)-3\left(\tau_{\mathrm{GI}}^{(1)}\right)^{2} .
$$


We have dropped those terms which represent integrated contributions and other second-order small-scale effects that can be distinguished from the large-scale part through their peculiar scale dependence. At this point, we make use of Einstein's equations. We take the expression for $\zeta^{(2)}$ in (36) and (37), and we use the $(0-0)$ component and the traceless part of the $(i-j)$ Einstein's equations (29) and (31) after having appropriately expanded the exponentials. Thus, on large scales we find that the temperature anisotropies are given by

$$
\frac{\Delta T_{\mathrm{GI}}^{(2)}}{T}=\frac{1}{18}\left(\phi_{\mathrm{GI}}^{(1)}\right)^{2}-\frac{\mathcal{K}}{10}-\frac{1}{10}\left[\zeta_{\mathrm{GI}}^{(2)}-2\left(\zeta_{\mathrm{GI}}^{(1)}\right)^{2}\right],
$$

where we have defined a kernel

$$
\mathcal{K}=10 \nabla^{-4} \partial_{i} \partial^{j}\left(\partial^{i} \psi^{(1)} \partial_{j} \psi^{(1)}\right)-\nabla^{-2}\left(\frac{10}{3} \partial^{i} \psi^{(1)} \partial_{i} \psi^{(1)}\right) .
$$

Notice that the factor $1 / 18$ nicely matches the corresponding term in (20). Equation (52) is the main result of this section. It clearly shows that there are two contributions to the final nonlinearity in the large-scale temperature anisotropies. The contribution, $\left[\zeta_{\mathrm{GI}}^{(2)}-2\left(\zeta_{\mathrm{GI}}^{(1)}\right)^{2}\right]$, comes from the "primordial" conditions set during or after inflation. They are encoded in the curvature perturbation $\zeta$ which remains constant once it has been generated. The remaining part of (52) describes the postinflation processing of the primordial non-Gaussian signal due to the nonlinear gravitational dynamics, including also second-order corrections at last scattering to the SachsWolfe effect $[32,51,59]$. Thus, (52) allows to neatly disentangle the primordial contribution to NG from that coming from that arising after inflation, and from it we can extract the nonlinearity parameter $f_{\mathrm{NL}}$, see the expression (39) which is usually adopted to phenomenologically parametrize the NG level of cosmological perturbations and has become the standard quantity to be observationally The definition of $f_{\mathrm{NL}}$ adopted in the analyses performed in [43] goes through the conventional Sachs-Wolfe formula $\Delta T / T=-\Phi / 3$ where $\Phi$ is Bardeen's potential, which is conventionally expanded as (up to a constant offset, which only affects the temperature monopole) $\Phi=\Phi_{\mathrm{L}}+f_{\mathrm{NL}}\left(\Phi_{\mathrm{L}}\right) \star\left(\Phi_{\mathrm{L}}\right)$. Therefore, using $\zeta^{(1)}=-(5 / 3) \psi_{\mathrm{GI}}^{(1)}$ during matter domination, from (52) we read the nonlinearity parameter in momentum space

$$
f_{\mathrm{NL}}\left(\mathbf{k}_{1}, \mathbf{k}_{2}\right)=-\left[\frac{5}{3}\left(1-a_{\mathrm{NL}}\right)+\frac{1}{6}-\frac{3}{10} \mathcal{K}\right],
$$

where

$$
\mathcal{K}=\frac{10\left(\mathbf{k}_{1} \cdot \mathbf{k}_{3}\right)\left(\mathbf{k}_{2} \cdot \mathbf{k}_{3}\right)}{k^{4}}-\frac{10}{3} \mathbf{k}_{1} \cdot \frac{\mathbf{k}_{2}}{k^{2}},
$$

with $\mathbf{k}_{3}+\mathbf{k}_{1}+\mathbf{k}_{2}=0$ and $k=\left|\mathbf{k}_{3}\right|$. To obtain (54) we have made use of the expression (38) to set the initial conditions. In particular, within the standard scenario where cosmological perturbations are due to the inflaton the primordial contribution to $\mathrm{NG}$ is given by $a_{\mathrm{NL}}=1-$ $(1 / 4)\left(n_{\zeta}-1\right)[6,7]$, where the spectral index is expressed in terms of the usual slow-roll parameters as $n_{\zeta}-1=-6 \epsilon+2 \eta$ [1]. The nonlinearity parameter from inflation now reads

$$
f_{\mathrm{NL}}^{\mathrm{inf}}=-\frac{5}{12}\left(n_{\zeta}-1\right)-\frac{1}{6}+\frac{3}{10} \mathcal{K}
$$

Therefore, the main NG contribution comes from the postinflation evolution of the second-order perturbations which give rise to order-one coefficients, while the primordial contribution is proportional to $\left|n_{\zeta}-1\right| \ll 1$. This is true even in the "squeezed" limit first discussed by Maldacena in [7], where one of the wavenumbers is much smaller than the other two, for example $k_{1} \ll k_{2,3}$ and $\mathcal{K} \rightarrow 0$.

\section{CMB Anisotropies at Second-Order at All Scales}

As we already mentioned in the Introduction, despite the importance of NG in CMB anisotropies, little effort has been made so far to provide accurate theoretical predictions of it. On the contrary, the vast majority of the literature has been devoted to the computation of the bispectrum of either the comovig curvature perturbation or the gravitational potential on large scales within given inflationary models. These, however, are not the physical quantities which are observed. One should instead provide a full prediction for the second-order radiation transfer function. A preliminary step towards this goal has been taken in [31, 32, 36, 59] (see also [37]) where the full second-order radiation transfer function for the CMB anisotropies on large angular scales in a flat universe filled with matter and cosmological constant was computed, including the second-order generalization of the Sachs-Wolfe effect, both the early and late integrated Sachs-Wolfe (ISW) effects and the contribution of the second-order tensor modes. We have partly reported about these works in the previous sections. (For some recent papers focusing on the generation and evolution of tensor perturbations at second-order see, e.g., [60-62].)

In this section we wish to offer a summary of some of the second-order effects in the CMB anisotropies on small scales. There are many sources of NG in CMB anisotropies, beyond the primordial one. The most relevant sources are the so-called secondary anisotropies, which arise after the last scattering epoch. These anisotropies can be divided into two categories: scattering secondaries, when the $\mathrm{CMB}$ photons scatter with electrons along the line of sight, and gravitational secondaries when effects are mediated by gravity [39]. Among the scattering secondaries, we may list the thermal Sunyaev-Zeldovich effect, where hot electrons in clusters transfer energy to the CMB photons, the kinetic Sunyaev-Zeldovich effect produced by the bulk motion of the electrons in clusters, the Ostriker-Vishniac effect, produced by bulk motions modulated by linear density perturbations, and effects due to reionization processes. The scattering secondaries are most significant on small angular scales as density inhomogeneities, bulk and thermal motions grow and become sizeable on small length-scales when structure formation proceeds. 
Gravitational secondaries arise from the change in energy of photons when the gravitational potential is timedependent, the ISW effect, and gravitational lensing. At late times, when the Universe becomes dominated by the dark energy, the gravitational potential on linear scales starts to decay, causing the ISW effect mainly on large angular scales. Other secondaries that result from a time dependent potential are the Rees-Sciama effect, produced during the matter-dominated epoch by the time evolution of the potential on nonlinear scales.

The fact that the potential never grows appreciably means that most second-order effects created by gravitational secondaries are generically small compared to those created by scattering ones. However, when a photon propagates from the last scattering to us, its path may be deflected because of the gravitational lensing. This effect does not create anisotropies, but only modifies existing ones. Since photons with large wavenumbers $k$ are lensed over many regions ( $\sim k / H$, where $H$ is the Hubble rate) along the line of sight, the corresponding second-order effect may be sizeable. The three-point function arising from the correlation of the gravitational lensing and ISW effects generated by the matter distribution along the line of sight $[40,41]$ and the SunyaevZeldovich effect [42] are large and detectable by Planck [43].

Another relevant source of NG comes from the physics operating at the recombination. A naive estimate would tell that these nonlinearities are tiny being suppressed by an extra power of the gravitational potential. However, the dynamics at recombination is quite involved because all the nonlinearities in the evolution of the baryon-photon fluid at recombination and the ones coming from general relativity should be accounted for. This complicated dynamics might lead to unexpected suppressions or enhancements of the NG at recombination. A step towards the evaluation of the threepoint correlation function has been taken in [45] where some effects were taken into account in the in so-called squeezed triangle limit, corresponding to the case when one wavenumber is much smaller than the other two and was outside the horizon at recombination (see however also [37] for a critical reassessment of some of the results contained in [45]. In particular notice that, contrary to what stated in [45], in the result (54), first obtained in [34], the contribution $(-1 / 6)$ is actually present and survives in the squeezed limit).

This section, which is based on $[46,47]$, presents the computation of the full system of Boltzmann equations, describing the evolution of the photon, baryon, and cold dark matter (CDM) fluids, at second order and neglecting polarization, These equations allow to follow the time evolution of the $\mathrm{CMB}$ anisotropies at second order on all angular scales from the early epochs, when the cosmological perturbations were generated, to the present time, through the recombination era. These calculations set the stage for the computation of the full second-order radiation transfer function at all scales and for a a generic set of initial conditions specifying the level of primordial nonGaussianity. Of course, on small angular scales, fully nonlinear calculations of specific effects like Sunyaev-Zel'dovich, gravitational lensing, and so forth would provide a more accurate estimate of the resulting CMB anisotropy, however, as long as the leading contribution to second-order statistics like the bispectrum is concerned, second-order perturbation theory suffices.

6.1. The Collisionless Boltzmann Equation for Photons. We are interested in the anisotropies in the cosmic distribution of photons and inhomogeneities in the matter. Photons are affected by gravity and by Compton scattering with free electrons. The latter are tightly coupled to protons. Both are, of course, affected by gravity. The metric which determines the gravitational forces is influenced by all these components plus CDM (and neutrinos). Our plan is to write down Boltzmann equations for the phase-space distributions of each species in the Universe.

The phase-space distribution of particles $g\left(x^{i}, P^{\mu}, \eta\right)$ is a function of spatial coordinates $x^{i}$, conformal time $\eta$, and momentum of the particle $P^{\mu}=d x^{\mu} / d \lambda$ where $\lambda$ parametrizes the particle path. Through the constraint $P^{2} \equiv g_{\mu \nu} P^{\mu} P^{v}=$ $-m^{2}$, where $m$ is the mass of the particle one can eliminate $P^{0}$ and $g\left(x^{i}, P^{j}, \eta\right)$ gives the number of particles in the differential phase-space volume $d x^{1} d x^{2} d x^{3} d P^{1} d P^{2} d P^{3}$. In the following, we will denote the distribution function for photons with $f$.

The photons' distribution evolves according to the Boltzmann equation

$$
\frac{d f}{d \eta}=\bar{C}[f],
$$

where the collision term is due to the scattering of photons off free electrons. In the following, we will derive the lefthand side of (57) while in the next section, we will compute the collision term.

For photons we can impose $P^{2} \equiv g_{\mu \nu} P^{\mu} P^{\nu}=0$ and using the metric (1) in the conformal time $\eta$ we find

$$
P^{2}=a^{2}\left[-e^{2 \Phi}\left(P^{0}\right)^{2}+\frac{p^{2}}{a^{2}}+2 \omega_{i} P^{0} P^{i}\right]=0,
$$

where we define

$$
p^{2}=g_{i j} P^{i} P^{j}
$$

From the constraint (58)

$$
P^{0}=e^{-\Phi}\left(\frac{p^{2}}{a^{2}}+2 \omega_{i} P^{0} P^{i}\right)^{1 / 2} .
$$

Notice that we immediately recover the usual zero and firstorder relations $P^{0}=p / a$ and $P^{0}=p\left(1-\Phi^{(1)}\right) / a$.

The components $P^{i}$ are proportional to $p n^{i}$, where $n^{i}$ is a unit vector with $n^{i} n_{i}=\delta_{i j} n^{i} n^{j}=1$. We can write $P^{i}=C n^{i}$, where $C$ is determined by

$$
g_{i j} P^{i} P^{j}=C^{2} a^{2}\left(e^{-2 \Psi}+\chi_{i j} n^{i} n^{j}\right)=p^{2},
$$

so that

$$
P^{i}=\frac{p}{a} n^{i}\left(e^{-2 \Psi}+\chi_{k m} n^{k} n^{m}\right)^{-1 / 2}=\frac{p}{a} n^{i} e^{\Psi}\left(1-\frac{1}{2} \chi_{k m} n^{k} n^{m}\right),
$$


where the last equality holds up to second order in the perturbations. Again, we recover the zero and first-order relations $P^{i}=p n^{i} / a$ and $P^{i}=p n^{i}\left(1+\Psi^{(1)}\right) / a$ respectively. Thus, up to second order we can write

$$
P^{0}=e^{-\Phi} \frac{P}{a}\left(1+\omega_{i} n^{i}\right) .
$$

Equation (62) and (63) allow us to replace $P^{0}$ and $P^{i}$ in terms of the variables $p$ and $n^{i}$. Therefore, as it is standard in the literature, from now on we will consider the phase-space distribution $f$ as a function of the momentum $\mathbf{p}=p n^{i}$ with magnitude $p$ and angular direction $n^{i}, f \equiv f\left(x^{i}, p, n^{i}, \eta\right)$. Notice that our $P^{i}$ are expressed via quantities, $p$ and $n^{i}$, which are different from the ones of [63]. However, the final Boltzmann equations that we obtain agree with the ones of [63]. The reason is that the differences in the evolution for $p$ emerge only at orders greater than two in the perturbative expansion in the fluctuations (at least if one neglects firstorder vector and tensor perturbation modes, as it is done in these computations), while the evolution equation for $n^{i}$ at linear-order (which is the one needed here, see below) is the same.

Thus, in terms of these variables, the total time derivative of the distribution function reads

$$
\frac{d f}{d \eta}=\frac{\partial f}{\partial \eta}+\frac{\partial f}{\partial x^{i}} \frac{d x^{i}}{d \eta}+\frac{\partial f}{\partial p} \frac{d p}{d \eta}+\frac{\partial f}{\partial n^{i}} \frac{d n^{i}}{d \eta} .
$$

In the following, we will compute $d x^{i} / d \eta, d p / d \eta$ and $d n^{i} / d \eta$.

(a) $d x^{i} / d \eta$ : From

$$
P^{i}=\frac{d x^{i}}{d \lambda}=\frac{d x^{i}}{d \eta} \frac{d \eta}{d \lambda}=\frac{d x^{i}}{d \eta} P^{0}
$$

and from (62) and (63)

$$
\frac{d x^{i}}{d \eta}=n^{i} e^{\Phi+\Psi}\left(1-\omega_{j} n^{j}-\frac{1}{2} \chi_{k m} n^{k} n^{m}\right) .
$$

(b) $d p / d \eta$ : For $d p / d \eta$, we make use of the time component of the geodesic equation $d P^{0} / d \lambda=-\Gamma_{\alpha \beta}^{0} P^{\alpha} P^{\beta}$, where $d / d \lambda=(d \eta / d \lambda) d / d \eta=P^{0} d / d \eta$, and

$$
\frac{d P^{0}}{d \eta}=-\Gamma_{\alpha \beta}^{0} \frac{P^{\alpha} P^{\beta}}{P^{0}}
$$

Using the metric (1), we find

$$
\begin{aligned}
2 \Gamma_{\alpha \beta}^{0} P^{\alpha} P^{\beta} \\
=g^{0 \nu}\left[2 \frac{\partial g_{\nu \alpha}}{\partial x^{\beta}}-\frac{\partial_{\alpha \beta}}{\partial x^{\nu}}\right] P^{\alpha} P^{\beta} \\
=2\left(\mathscr{H}+\Phi^{\prime}\right)\left(P^{0}\right)^{2}+4 \Phi_{, i} P^{0} P^{i}+4 \mathscr{H} \omega_{i} P^{0} P^{i} \\
\quad+2 e^{-2 \Phi}\left[\left(\mathscr{H}-\Psi^{\prime}\right) e^{-2 \Psi} \delta_{i j}-\omega_{i, j}+\frac{1}{2} \chi_{i j}^{\prime}+\mathscr{H} \chi_{i j}\right] P^{i} P^{j}
\end{aligned}
$$

On the other hand (63) of $P^{0}$ in terms of $p$ and $n^{i}$ gives

$$
\begin{aligned}
\frac{d P^{0}}{d \eta}= & -\frac{p}{a} \frac{d \Phi}{d \eta} e^{-\Phi}\left(1+\omega_{i} n^{i}\right)+e^{-\Phi}\left(1+\omega_{i} n^{i}\right) \frac{d(p / a)}{d \eta} \\
& +\frac{p}{a} e^{-\Phi} \frac{d\left(\omega_{i} n^{i}\right)}{d \eta} .
\end{aligned}
$$

Thus, (67) allows us express $d p / d \eta$ as

$$
\frac{1}{p} \frac{d p}{d \eta}=-\mathscr{H}+\Psi^{\prime}-\Phi_{, i} n^{i} e^{\Phi+\Psi}-\omega_{i}^{\prime} n^{i}-\frac{1}{2} \chi_{i j}^{\prime} n^{i} n^{j},
$$

where in (68) we have replaced $P^{0}$ and $P^{i}$ by (63) and (62). Notice that in order to obtain (70), we have used the following expressions for the total time derivatives of the metric perturbations

$$
\begin{aligned}
\frac{d \Phi}{d \eta} & =\frac{\partial \Phi}{\partial \eta}+\frac{\partial \Phi}{\partial x^{i}} \frac{d x^{i}}{d \eta} \\
& =\frac{\partial \Phi}{\partial \eta}+\frac{\partial \Phi}{\partial x^{i}} n^{i} e^{\Phi+\Psi}\left(1-\omega_{j} n^{j}-\frac{1}{2} \chi_{k m} n^{k} n^{m}\right)
\end{aligned}
$$

$$
\begin{aligned}
\frac{d\left(\omega_{i} n^{i}\right)}{d \eta} & =n^{i}\left(\frac{\partial \omega_{i}}{\partial \eta}+\frac{\partial \omega_{i}}{\partial x^{j}} \frac{d x^{j}}{d \eta}\right) \\
& =\frac{\partial \omega_{i}}{\partial \eta} n^{i}+\frac{\partial \omega_{i}}{\partial x^{j}} n^{i} n^{j}
\end{aligned}
$$

where we have taken into account that $\omega_{i}$ is already a secondorder perturbation so that we can neglect $d n^{i} / d \eta$ which is at least a first order quantity, and we can take the zero-order expression in (66), $d x^{i} / d \eta=n^{i}$. In fact, there is also an alternative expression for $d p / d \eta$ which turns out to be useful later and which can be obtained by applying once more (71)

$$
\frac{1}{p} \frac{d p}{d \eta}=-\mathscr{H}-\frac{d \Phi}{d \eta}+\Phi^{\prime}+\Psi^{\prime}-\omega_{i}^{\prime} n^{i}-\frac{1}{2} \chi_{i j}^{\prime} n^{i} n^{j} .
$$

(c) $d n^{i} / d \eta$ : We can proceed in a similar way to compute $d n^{i} / d \eta$. Notice that since in (64) it multiplies $\partial f / \partial n^{i}$ which is first order, we need only the first order perturbation of $d n^{i} / d \eta$. We use the spatial components of the geodesic equations $d P^{i} / d \lambda=-\Gamma_{\alpha \beta}^{i} P^{\alpha} P^{\beta}$ written as

$$
\frac{d P^{i}}{d \eta}=-\Gamma_{\alpha \beta}^{i} \frac{P^{\alpha} P^{\beta}}{P^{0}}
$$


For the right-hand side, we find, up to second order,

$$
\begin{aligned}
2 \Gamma_{\alpha \beta}^{i} P^{\alpha} P^{\beta}= & g^{i \nu}\left[\frac{\partial g_{\alpha \nu}}{\partial x^{\beta}}+\frac{\partial g_{\beta \nu}}{\partial x^{\alpha}}-\frac{\partial g_{\alpha \beta}}{\partial x^{\nu}}\right] P^{\alpha} P^{\beta} \\
= & 4\left(\mathscr{H}-\Psi^{\prime}\right) P^{i} P^{0}+2\left(\chi_{k}^{i^{\prime}}+\omega_{, k}^{i}-\omega_{k}^{i}\right) P^{0} P^{k} \\
& +\left(2 \frac{\partial \Phi}{\partial x^{i}} e^{2 \Phi+2 \Psi}+2 \omega^{i^{\prime}}+2 \mathcal{H} \omega^{i}\right)\left(P^{0}\right)^{2} \\
& -4 \frac{\partial \Psi}{\partial x^{k}} P^{i} P^{k}+2 \frac{\partial \Psi}{\partial x^{i}} \delta_{k m} P^{k} P^{m} \\
& -\left[2 \mathscr{H} \omega^{i} \delta_{j k}-\left(\frac{\partial \chi_{j}^{i}}{\partial x^{k}}+\frac{\partial \chi_{k}^{i}}{\partial x^{j}}-\frac{\partial \chi_{j k}}{\partial x_{i}}\right)\right] P^{j} P^{k}
\end{aligned}
$$

while the expression (62) of $P^{i}$ in terms of our variables $p$ and $n^{i}$ in the left-hand side of (74) brings

$$
\begin{aligned}
\frac{d P^{i}}{d \eta}= & \frac{p}{a} e^{\Psi}\left[\frac{d \Psi}{d \eta} n^{i}+\frac{a}{p} \frac{d(p / a)}{d \eta} n^{i}+\frac{d n^{i}}{d \eta}\right]\left(1-\frac{1}{2} \chi_{k m} n^{k} n^{m}\right) \\
& -\frac{p}{a} n^{i} e^{\Psi} \frac{1}{2} \frac{\mathrm{d}\left(\chi_{k m} n^{k} n^{m}\right)}{d \eta} .
\end{aligned}
$$

Thus, using the expression (62) for $P^{i}$ and (60) for $P^{0}$ in (75), together with the previous result (70), the geodesic equation (74) gives the following expression $d n^{i} / d \eta$ (valid up to first order)

$$
\frac{d n^{i}}{d \eta}=\left(\Phi_{, k}+\Psi_{, k}\right) n^{k} n^{i}-\Phi^{, i}-\Psi^{, i}
$$

To proceed further we now expand the distribution function for photons around the zero-order value $f^{(0)}$ which is that of a Bose-Einstein distribution

$$
f^{(0)}(p, \eta)=2 \frac{1}{\exp \{p /(T(\eta))\}-1},
$$

where $T(\eta)$ is the average (zero-order) temperature and the factor 2 comes from the spin degrees of photons. The perturbed distribution of photons will depend also on $x^{i}$ and on the propagation direction $n^{i}$ so as to account for inhomogeneities and anisotropies

$$
\begin{aligned}
f\left(x^{i}, p, n^{i}, \eta\right)= & f^{(0)}(p, \eta)+f^{(1)}\left(x^{i}, p, n^{i}, \eta\right) \\
& +\frac{1}{2} f^{(2)}\left(x^{i}, p, n^{i}, \eta\right)
\end{aligned}
$$

where we split the perturbation of the distribution function into a first and a second-order part. The Boltzmann equation up to second order can be written in a straightforward way by recalling that the total time derivative of a given $i$ th perturbation, as for example, $d f^{(i)} / d \eta$ is at least a quantity of the $i$-th order. Thus, it is easy to realize, looking at (64), that the left-hand side of Boltzmann equation can be written up to second order as

$$
\begin{aligned}
\frac{d f}{d \eta}= & \frac{d f^{(1)}}{d \eta}+\frac{1}{2} \frac{d f^{(2)}}{d \eta}-p \frac{\partial f^{(0)}}{\partial p} \frac{d}{d \eta}\left(\Phi^{(1)}+\frac{1}{2} \Phi^{(2)}\right) \\
& +p \frac{\partial f^{(0)}}{\partial p} \frac{\partial}{\partial \eta}\left(\Phi^{(1)}+\Psi^{(1)}+\frac{1}{2} \Phi^{(2)}+\frac{1}{2} \Psi^{(2)}\right) \\
& -p \frac{\partial f^{(0)}}{\partial p} \frac{\partial \omega_{i}}{\partial \eta} n^{i}-\frac{1}{2} p \frac{\partial f^{(0)}}{\partial p} \frac{\partial \chi_{i j}}{\partial \eta} n^{i} n^{j},
\end{aligned}
$$

where for simplicity in (80), we have already used the background Boltzmann equation $\left.(d f / d \eta)\right|^{(0)}=0$. In (80) there are all the terms which will give rise to the integrated Sachs-Wolfe effects (corresponding to the terms which explicitly depend on the gravitational perturbations), while other effects, such as the gravitational lensing, are still hidden in the (second-order part) of the first term. In fact in order to obtain (80) we just need for the time being to know the expression for $d p / d \eta$, (73).

\subsection{Collision Term}

6.2.1. The Collision Integral. In this section, we focus on the collision term due to Compton scattering (notice that in this section all the quantities and their indices are meant to be defined in the local Minkowski frame)

$$
e(\mathbf{q}) \gamma(\mathbf{p}) \longleftrightarrow e\left(\mathbf{q}^{\prime}\right) \gamma\left(\mathbf{p}^{\prime}\right),
$$

where we have indicated the momentum of the photons and electrons involved in the collisions. The collision term will be important for small scale anisotropies and spectral distortions. The important point to compute the collision term is that for the epoch of interest very little energy is transferred. Therefore, one can proceed by expanding the right hand side of (57) both in the small perturbation, (79), and in the small energy transfer.

The collision term is given (up to second order) by

$$
\bar{C}(\mathbf{p})=C(\mathbf{p}) a e^{\Phi},
$$

where $a$ is the scale factor and

$$
\begin{aligned}
C(\mathbf{p})= & \frac{1}{E(\mathbf{p})} \int \frac{d \mathbf{q}}{(2 \pi)^{3} 2 E(\mathbf{q})} \frac{d \mathbf{q}^{\prime}}{(2 \pi)^{3} 2 E\left(\mathbf{q}^{\prime}\right)} \frac{d \mathbf{p}^{\prime}}{(2 \pi)^{3} 2 E\left(\mathbf{p}^{\prime}\right)} \\
& \times(2 \pi)^{4} \delta^{4}\left(q+p-q^{\prime}-p^{\prime}\right)|M|^{2} \\
& \times\left\{g\left(\mathbf{q}^{\prime}\right) f\left(\mathbf{p}^{\prime}\right)[1+f(\mathbf{p})]-g(\mathbf{q}) f(\mathbf{p})\left[1+f\left(\mathbf{p}^{\prime}\right)\right]\right\}
\end{aligned}
$$

where $E(\mathbf{q})=\left(q^{2}+m_{e}^{2}\right)^{1 / 2}, M$ is the amplitude of the scattering process, $\delta^{4}\left(q+p-q^{\prime}-p^{\prime}\right)=\delta^{3}\left(\mathbf{q}+\mathbf{p}-\mathbf{q}^{\prime}-\right.$ $\left.\mathbf{p}^{\prime}\right) \delta\left(E(\mathbf{q})+p-E\left(\mathbf{q}^{\prime}\right)-p^{\prime}\right)$ ensures the energy-momentum conservation and $g$ is the distribution function for electrons. The Pauli suppression factors $(1-g)$ have been dropped since for the epoch of interest the density of electrons $n_{e}$ is low. The reason why we write the collision term as in (82) 
is that the starting point of the Boltzmann equation requires differentiation with respect to an affine parameter $\lambda, d f / d \lambda=$ $C^{\prime}$. In moving to the conformal time $\eta$, one rewrites the Boltzmann equation as $d f / d \eta=C^{\prime}\left(P^{0}\right)^{-1}$, with $P^{0}=d \eta / d \lambda$ given by (63). Taking into account that the collision term is at least of first order, (82) then follows. The electrons are kept in thermal equilibrium by Coulomb interactions with protons and they are nonrelativistic, thus we can take a MaxwellBoltzmann distribution around some bulk velocity $\mathbf{v}$

$$
g(\mathbf{q})=n_{e}\left(\frac{2 \pi}{m_{e} T_{e}}\right)^{3 / 2} \exp \left\{-\frac{\left(\mathbf{q}-m_{e} \mathbf{v}\right)^{2}}{2 m_{e} T_{e}}\right\}
$$

By using the three dimensional delta function the energy transfer is given by $E(\mathbf{q})-E\left(\mathbf{q}+\mathbf{p}-\mathbf{p}^{\prime}\right)$ and it turns out to be small compared to the typical thermal energies

$$
E(\mathbf{q})-E\left(\mathbf{q}+\mathbf{p}-\mathbf{p}^{\prime}\right) \simeq \frac{\left(\mathbf{p}-\mathbf{p}^{\prime}\right) \cdot \mathbf{q}}{m_{e}}=\mathcal{O}\left(\frac{T q}{m_{e}}\right) .
$$

In (85), we have used $E(\mathbf{q})=m_{e}+q^{2} / 2 m_{e}$ and the fact that, since the scattering is almost elastic $\left(p \simeq p^{\prime}\right),\left(\mathbf{p}-\mathbf{p}^{\prime}\right)$ is of order $p \sim T$, with $q$ much bigger than $\left(\mathbf{p}-\mathbf{p}^{\prime}\right)$. In general, the electron momentum has two contributions, the bulk velocity $\left(q=m_{e} v\right)$ and the thermal motion $\left(q \sim\left(m_{e} T\right)^{1 / 2}\right)$ and thus the parameter expansion $q / m_{e}$ includes the small bulk velocity $\mathbf{v}$ and the ratio $\left(T / m_{e}\right)^{1 / 2}$ which is small because the electrons are nonrelativistic.

The expansion of all the quantities entering the collision term in the energy transfer parameter and the integration over the momenta $\mathbf{q}$ and $\mathbf{q}^{\prime}$ is described in details in [64]. It is easy to realize that we just need the scattering amplitude up to first order since at zero-order $g\left(\mathbf{q}^{\prime}\right)=g\left(\mathbf{q}+\mathbf{p}-\mathbf{p}^{\prime}\right)=g(\mathbf{q})$ and $\delta\left(E(\mathbf{q})+p-E\left(\mathbf{q}^{\prime}\right)-p^{\prime}\right)=\delta\left(p-p^{\prime}\right)$ so that all the zero-order quantities multiplying $|M|^{2}$ vanish. To first order

$$
|M|^{2}=6 \pi \sigma_{T} m_{e}^{2}\left[\left(1+\cos ^{2} \theta\right)-2 \cos \theta(1-\cos \theta) \mathbf{q} \cdot \frac{\left(\hat{p}+\hat{p}^{\prime}\right)}{m_{e}}\right],
$$

where $\cos \theta=n \cdot n^{\prime}$ is the scattering angle and $\sigma_{T}$ the Thompson cross-section. The resulting collision term up to second order is given by [64]

$$
\begin{array}{r}
C(\mathbf{p})=\frac{3 n_{e} \sigma_{T}}{4 p} \int d p^{\prime} p^{\prime} \frac{d \Omega^{\prime}}{4 \pi}\left[c^{(1)}\left(\mathbf{p}, \mathbf{p}^{\prime}\right)+c_{\Delta}^{(2)}\left(\mathbf{p}, \mathbf{p}^{\prime}\right)\right. \\
+c_{v}^{(2)}\left(\mathbf{p}, \mathbf{p}^{\prime}\right)+c_{\Delta v}^{(2)}\left(\mathbf{p}, \mathbf{p}^{\prime}\right) \\
\left.+c_{v v}^{(2)}\left(\mathbf{p}, \mathbf{p}^{\prime}\right)+c_{K}^{(2)}\left(\mathbf{p}, \mathbf{p}^{\prime}\right)\right],
\end{array}
$$

where we arrange the different contributions following [64]. The first order term reads

$$
\begin{aligned}
c^{(1)}\left(\mathbf{p}, \mathbf{p}^{\prime}\right) & \\
=(1+ & \left.\cos ^{2} \theta\right) \\
\times & {\left[\delta\left(p-p^{\prime}\right)\left(f^{(1)}\left(\mathbf{p}^{\prime}\right)-f^{(1)}(\mathbf{p})\right)\right.} \\
& \left.\quad+\left(f^{(0)}\left(p^{\prime}\right)-f^{(0)}(p)\right)\left(\mathbf{p}-\mathbf{p}^{\prime}\right) \cdot \mathbf{v} \frac{\partial \delta\left(p-p^{\prime}\right)}{\partial p^{\prime}}\right],
\end{aligned}
$$

while the second-order terms have been separated into four parts. There is the so-called anisotropy suppression term

$$
c_{\Delta}^{(2)}\left(\mathbf{p}, \mathbf{p}^{\prime}\right)=\frac{1}{2}\left(1+\cos ^{2} \theta\right) \delta\left(p-p^{\prime}\right)\left(f^{(2)}\left(\mathbf{p}^{\prime}\right)-f^{(2)}(\mathbf{p})\right) ;
$$

a term which depends on the second-order velocity perturbation defined by the expansion of the bulk flow as $\mathbf{v}=$ $\mathbf{v}^{(1)}+\mathbf{v}^{(2)} / 2$

$$
\begin{aligned}
c_{v}^{(2)}\left(\mathbf{p}, \mathbf{p}^{\prime}\right)= & \frac{1}{2}\left(1+\cos ^{2} \theta\right)\left(f^{(0)}\left(p^{\prime}\right)-f^{(0)}(p)\right)\left(\mathbf{p}-\mathbf{p}^{\prime}\right) \\
& \cdot \mathbf{v}^{(2)} \frac{\partial \delta\left(p-p^{\prime}\right)}{\partial p^{\prime}} ;
\end{aligned}
$$

a set of terms coupling the photon perturbation to the velocity

$$
\begin{aligned}
& c_{\Delta v}^{(2)}\left(\mathbf{p}, \mathbf{p}^{\prime}\right) \\
& =\left(f^{(1)}\left(\mathbf{p}^{\prime}\right)-f^{(1)}(\mathbf{p})\right) \\
& \quad \times\left[\left(1+\cos ^{2} \theta\right)\left(\mathbf{p}-\mathbf{p}^{\prime}\right) \cdot \mathbf{v} \frac{\partial \delta\left(p-p^{\prime}\right)}{\partial p^{\prime}}\right. \\
& \left.\quad-2 \cos \theta(1-\cos \theta) \delta\left(p-p^{\prime}\right)\left(\mathbf{n}+\mathbf{n}^{\prime}\right) \cdot \mathbf{v}\right],
\end{aligned}
$$

and a set of source terms quadratic in the velocity

$$
\begin{aligned}
c_{v v}^{(2)}\left(\mathbf{p}, \mathbf{p}^{\prime}\right)= & \left(f^{(0)}\left(p^{\prime}\right)-f^{(0)}(p)\right)\left(\mathbf{p}-\mathbf{p}^{\prime}\right) \cdot \mathbf{v} \\
\times & {\left[\left(1+\cos ^{2} \theta\right) \frac{\left(\mathbf{p}-\mathbf{p}^{\prime}\right) \cdot \mathbf{v}}{2} \frac{\partial^{2} \delta\left(p-p^{\prime}\right)}{\partial p^{\prime 2}}\right.} \\
& \left.\quad-2 \cos \theta(1-\cos \theta)\left(\mathbf{n}+\mathbf{n}^{\prime}\right) \cdot \mathbf{v} \frac{\partial \delta\left(p-p^{\prime}\right)}{\partial p^{\prime}}\right] .
\end{aligned}
$$


The last contribution are the Kompaneets terms describing spectral distortions to the $\mathrm{CMB}$

$$
\begin{aligned}
c_{K}^{(2)}\left(\mathbf{p}, \mathbf{p}^{\prime}\right) & \\
= & \left(1+\cos ^{2} \theta\right) \frac{\left(\mathbf{p}-\mathbf{p}^{\prime}\right)^{2}}{2 m_{e}} \\
\times & {\left[\left(f^{(0)}\left(p^{\prime}\right)-f^{(0)}(p)\right) T_{e} \frac{\partial^{2} \delta\left(p-p^{\prime}\right)}{\partial p^{\prime 2}}\right.} \\
& \left.-\left(f^{(0)}\left(p^{\prime}\right)+f^{(0)}(p)+2 f^{(0)}\left(p^{\prime}\right) f^{(0)}(p)\right) \frac{\partial \delta\left(p-p^{\prime}\right)}{\partial p^{\prime}}\right] \\
+ & \frac{2\left(p-p^{\prime}\right) \cos \theta\left(1-\cos ^{2} \theta\right)}{m_{e}} \\
\times & {\left[\delta\left(p-p^{\prime}\right) f^{(0)}\left(p^{\prime}\right)\left(1+f^{(0)}(p)\right)\left(f^{(0)}\left(p^{\prime}\right)-f^{(0)}(p)\right)\right.} \\
& \left.\times \frac{\partial \delta\left(p-p^{\prime}\right)}{\partial p^{\prime}}\right] .
\end{aligned}
$$

Let us make a couple of comments about the various contributions to the collision term. First, notice the term $c_{v}^{(2)}\left(\mathbf{p}, \mathbf{p}^{\prime}\right)$ due to second-order perturbations in the velocity of electrons which is absent in [64]. In standard cosmological scenarios (like inflation), vector perturbations are not generated at linear order, so that linear velocities are irrotational $v^{(1) i}=$ $\partial^{i} v^{(1)}$. However, at second order vector perturbations are generated after horizon crossing as nonlinear combinations of primordial scalar modes. Thus, we must take into account also a transverse (divergence-free) component, $v^{(2) i}=\partial^{i} v^{(2)}+$ $v_{T}^{(2) i}$ with $\partial_{i} v_{T}^{(2) i}=0$. As we will see, such vector perturbations will break azimuthal symmetry of the collision term with respect to a given mode $\mathbf{k}$, which instead usually holds at linear order. Secondly, notice that the number density of electrons appearing in (87) must be expanded as $n_{e}=\bar{n}_{e}(1+$ $\left.\delta_{e}\right)$ and then

$$
\delta_{e}^{(1)} c^{(1)}\left(\mathbf{p}, \mathbf{p}^{\prime}\right)
$$

gives rise to second-order contributions in addition to the list above, where we split $\delta_{e}=\delta_{e}^{(1)}+\delta_{e}^{(2)} / 2$ into a first- and second-order part. In particular, the combination with the term proportional to $\mathbf{v}$ in $c^{(1)}\left(\mathbf{p}, \mathbf{p}^{\prime}\right)$ gives rise to the so-called Vishniac effect, as discussed in [64].

\subsubsection{Computation of Different Contributions to the Collision} Term. In the integral (87) over the momentum $\mathbf{p}^{\prime}$ the firstorder term gives the usual collision term

$$
\begin{aligned}
& C^{(1)}(\mathbf{p}) \\
& =n_{e} \sigma_{T}\left[f_{0}^{(1)}(p)+\frac{1}{2} f_{2}^{(1)} P_{2}(\hat{\mathbf{v}} \cdot \mathbf{n})-f^{(1)}-p \frac{\partial f^{(0)}}{\partial p} \mathbf{v} \cdot \mathbf{n}\right],
\end{aligned}
$$

where one uses the decomposition in Legendre polynomials

$$
f^{(1)}(\mathbf{x}, p, \mathbf{n})=\sum_{\ell}(2 \ell+1) f_{\ell}^{(1)}(p) P_{\ell}(\cos \vartheta),
$$

where $\vartheta$ is the polar angle of $\mathbf{n}, \cos \vartheta=\mathbf{n} \cdot \widehat{\mathbf{v}}$.

In the following, we compute the second-order collision term separately for the different contributions, using the notation $C(\mathbf{p})=C^{(1)}(\mathbf{p})+C^{(2)}(\mathbf{p}) / 2$. We have not reported the details of the calculation of the first-order term because for its second-order analog, $c_{\Delta}^{(2)}\left(\mathbf{p}, \mathbf{p}^{\prime}\right)+c_{v}^{(2)}\left(\mathbf{p}, \mathbf{p}^{\prime}\right)$, the procedure is the same. The important difference is that the second-order velocity term includes a vector part, and this leads to a generic angular decomposition of the distribution function (for simplicity drop the time dependence)

$$
f^{(i)}(\mathbf{x}, p, \mathbf{n})=\sum_{\ell} \sum_{m=-\ell}^{\ell} f_{\ell m}^{(i)}(\mathbf{x}, p)(-i)^{\ell} \sqrt{\frac{4 \pi}{2 \ell+1}} Y_{\ell m}(\mathbf{n}),
$$

such that

$$
f_{\ell m}^{(i)}=(-i)^{-\ell} \sqrt{\frac{2 \ell+1}{4 \pi}} \int d \Omega f^{(i)} Y_{\ell m}^{*}(\mathbf{n}) .
$$

Such a decomposition holds also in Fourier space. The notation at this stage is a bit confusing, so let us restate it: superscripts denote the order of the perturbation; the subscripts refer to the moments of the distribution. Indeed, at first order, one can drop the dependence on $m$ setting $m=0$ using the fact that the distribution function does not depend on the azimuthal angle $\phi$. In this case, the relation with $f_{l}^{(1)}$ is

$$
f_{\ell m}^{(1)}=(-i)^{-\ell}(2 \ell+1) \delta_{m 0} f_{\ell}^{(1)}
$$

(a) $c_{\Delta}^{(2)}\left(\mathbf{p}, \mathbf{p}^{\prime}\right)$ :

The integral over $\mathbf{p}^{\prime}$ yields

$$
\begin{aligned}
C_{\Delta}^{(2)}(\mathbf{p}) & =\frac{3 n_{e} \sigma_{T}}{4 p} \int d p^{\prime} p^{\prime} \frac{d \Omega^{\prime}}{4 \pi} c_{\Delta}^{(2)}\left(\mathbf{p}, \mathbf{p}^{\prime}\right) \\
& =\frac{3 n_{e} \sigma_{T}}{4 p} \int d p^{\prime} p^{\prime} \delta\left(p-p^{\prime}\right),
\end{aligned}
$$

To perform the angular integral we write the angular dependence on the scattering angle $\cos \theta=\mathbf{n} \cdot \mathbf{n}^{\prime}$ in terms of the Legendre polynomials

$$
\begin{aligned}
{\left[1+\left(\mathbf{n} \cdot \mathbf{n}^{\prime}\right)^{2}\right] } & =\frac{4}{3}\left[1+\frac{1}{2} P_{2}\left(\mathbf{n} \cdot \mathbf{n}^{\prime}\right)\right] \\
& =\left[1+\frac{1}{2} \sum_{m=-2}^{2} Y_{2 m}(n) Y_{2 m}^{*}\left(\mathbf{n}^{\prime}\right) \frac{4 \pi}{2 \ell+1}\right],
\end{aligned}
$$

where in the last step we used the addition theorem for spherical harmonics

$$
P_{\ell}=\frac{4 \pi}{2 \ell+1} \sum_{m=-2}^{2} Y_{\ell m}(\mathbf{n}) Y_{\ell m}^{*}\left(\mathbf{n}^{\prime}\right)
$$


Using the decomposition (98) and the orthonormality of the spherical harmonics, we find

$$
\begin{aligned}
C_{\Delta}^{(2)}(\mathbf{p})=n_{e} \sigma_{T}[ & f_{00}^{(2)}(\mathbf{p})-f^{(2)}(\mathbf{p}) \\
& \left.\quad-\frac{1}{2} \sum_{m=-2}^{2} \frac{\sqrt{4 \pi}}{5^{3 / 2}} f_{2 m}^{(2)}(p) Y_{2 m}(\mathbf{n})\right] .
\end{aligned}
$$

It is easy to recover the result for the corresponding firstorder contribution in (229) by using (205).

(b) $c_{v}^{(2)}\left(\mathbf{p}, \mathbf{p}^{\prime}\right)$ :

Let us fix for simplicity our coordinates such that the polar angle of $\mathbf{n}^{\prime}$ is defined by $\mu^{\prime}=\widehat{\mathbf{v}}^{(2)} \cdot \mathbf{n}^{\prime}$ with $\phi^{\prime}$ the corresponding azimuthal angle. The contribution of $c_{v}^{(2)}\left(\mathbf{p}, \mathbf{p}^{\prime}\right)$ to the collision term is then

$$
\begin{aligned}
C_{v}^{(2)}(\mathbf{p})= & \frac{3 n_{e} \sigma_{T}}{4 p} v^{(2)} \int d p^{\prime} p^{\prime}\left[f^{(0)}\left(p^{\prime}\right)-f^{(0)}(p)\right] \\
& \times \frac{\partial \delta\left(p-p^{\prime}\right)}{\partial p^{\prime}} \\
& \times \int_{-1}^{1} \frac{d \mu^{\prime}}{2}\left(p \mu-p^{\prime} \mu^{\prime}\right) \int_{0}^{2 \pi} \frac{d \phi^{\prime}}{2 \pi}\left[1+\left(\mathbf{p} \cdot \mathbf{p}^{\prime}\right)^{2}\right] .
\end{aligned}
$$

We can use (101) which in our coordinate system reads

$$
\frac{4}{3}\left[1+\frac{1}{2} \sum_{m=-2}^{m} \frac{(2-m) !}{(2+m) !} P_{2}^{m}\left(\mathbf{n} \cdot \widehat{\mathbf{v}}^{(2)}\right) P_{2}^{m}\left(\mathbf{n}^{\prime} \cdot \widehat{\mathbf{v}}^{(2)}\right) e^{i m\left(\phi^{\prime}-\phi\right)}\right]
$$

so that

$$
\begin{aligned}
\int \frac{d \phi^{\prime}}{2 \pi} P_{2}\left(\mathbf{n} \cdot \mathbf{n}^{\prime}\right) & =P_{2}\left(\mathbf{n} \cdot \hat{\mathbf{v}}^{(2)}\right) P_{2}\left(\mathbf{n}^{\prime} \cdot \hat{\mathbf{v}}^{(2)}\right) \\
& =P_{2}(\mu) P_{2}\left(\mu^{\prime}\right) .
\end{aligned}
$$

By using the orthonormality of the Legendre polynomials and integrating by parts over $p^{\prime}$, we find

$$
C_{v}^{(2)}(\mathbf{p})=-n_{e} \sigma_{T} p \frac{\partial f^{(0)}}{\partial p} \mathbf{v}^{(2)} \cdot \mathbf{n} .
$$

As it is clear by the presence of the scalar product $\mathbf{v}^{(2)} \cdot \mathbf{p}$, the final result is independent of the coordinates chosen. (c) $c_{\Delta v}^{(2)}\left(\mathbf{p}, \mathbf{p}^{\prime}\right)$ :

let us consider the contribution from the first term

$$
\begin{aligned}
& c_{\Delta v(I)}^{(2)}\left(\mathbf{p}, \mathbf{p}^{\prime}\right) \\
& =\left(1+\cos ^{2} \theta\right)\left(f^{(1)}\left(\mathbf{p}^{\prime}\right)-f^{(1)}(\mathbf{p})\right)\left(\mathbf{p}-\mathbf{p}^{\prime}\right) \cdot \frac{\mathbf{v} \partial \delta\left(p-p^{\prime}\right)}{\partial p^{\prime}},
\end{aligned}
$$

where the velocity has to be considered at first order. In the integral (87), it brings

$$
\begin{aligned}
\frac{1}{2} C_{\Delta v(I)}^{(2)}= & \frac{3 n_{e} \sigma_{T} v}{4 p} \int d p^{\prime} p^{\prime} \frac{\partial \delta\left(p-p^{\prime}\right)}{\partial p^{\prime}} \\
& \times \int_{-1}^{1} \frac{d \mu^{\prime}}{2}\left[f^{(1)}\left(\mathbf{p}^{\prime}\right)-f^{(1)}(\mathbf{p})\right] \\
& \times\left(p \mu-p^{\prime} \mu^{\prime}\right) \int_{0}^{2 \pi} \frac{d \phi^{\prime}}{2 \pi}\left(1+\cos ^{2} \theta\right) .
\end{aligned}
$$

The procedure to do the integral is the same as above. We use the same relations as in (105) and (106), where now the angles are those taken with respect to the first-order velocity. This eliminates the integral over $\phi^{\prime}$, and integrating by parts over $p^{\prime}$ yields

$$
\begin{aligned}
\frac{1}{2} C_{\Delta v(I)}^{(2)}(\mathbf{p})= & -\frac{3 n_{e} \sigma_{T} v}{4 p} \int_{-1}^{1} \frac{d \mu^{\prime}}{2}\left[\frac{4}{3}+\frac{2}{3} P_{2}(\mu) P_{2}\left(\mu^{\prime}\right)\right] \\
\times & {\left[p\left(\mu-2 \mu^{\prime}\right)\left(f^{(1)}\left(p, \mu^{\prime}\right)-f^{(1)}(p, \mu)\right)\right.} \\
& \left.+p^{2}\left(\mu-\mu^{\prime}\right) \frac{\partial f^{(1)}\left(p, \mu^{\prime}\right)}{\partial p}\right] .
\end{aligned}
$$

We now use the decomposition (96) and the orthonormality of the Legendre polynomials to find

$$
\begin{aligned}
& \int \frac{d \mu^{\prime}}{2} \mu^{\prime} f^{(1)}\left(p, \mu^{\prime}\right) P_{2}\left(\mu^{\prime}\right) \\
& =\sum_{\ell} \int \frac{d \mu^{\prime}}{2} \mu^{\prime} P_{2}\left(\mu^{\prime}\right) P_{l}\left(\mu^{\prime}\right) f_{\ell}^{(1)}(p) \\
& =\sum_{\ell} \int \frac{d \mu^{\prime}}{2}\left[\frac{2}{5} P_{1}\left(\mu^{\prime}\right)+\frac{3}{5} P_{3}\left(\mu^{\prime}\right)\right] P_{\ell}\left(\mu^{\prime}\right) f_{\ell}^{(1)}(p) \\
& =\frac{2}{5} f_{1}^{(1)}(p)+\frac{3}{5} f_{3}^{(1)}(p),
\end{aligned}
$$


where we have used $\mu^{\prime} P_{2}\left(\mu^{\prime}\right) P_{l}\left(\mu^{\prime}\right)=(2 / 5) P_{1}\left(\mu^{\prime}\right)+$ (3/5) $P_{3}\left(\mu^{\prime}\right)$, with $P_{1}\left(\mu^{\prime}\right)=\mu^{\prime}$. Thus, from (110) we get

$$
\begin{aligned}
& \frac{1}{2} C_{\Delta v(I)}^{(2)}(\mathbf{p}) \\
&=n_{e} \sigma_{T}\left\{\mathbf { v } \cdot \mathbf { n } \left[f^{(1)}(\mathbf{p})-f_{0}^{(1)}(p)-p \frac{\partial f_{0}^{(1)}(p)}{\partial p}\right.\right. \\
&\left.-\frac{1}{2} P_{2}(\hat{v} \cdot \mathbf{n})\left(f_{2}^{(1)}(p)+p \frac{\partial f_{2}^{(1)}(p)}{\partial p}\right)\right] \\
&+v {\left[2 f_{1}^{(1)}(p)+p \frac{\partial f_{1}^{(1)}(p)}{\partial p}+\frac{1}{5} P_{2}(\hat{\mathbf{v}} \cdot \mathbf{n})\right.} \\
& \times\left(2 f_{1}^{(1)}(p)+p \frac{\partial f_{1}^{(1)}(p)}{\partial p}\right. \\
&\left.\left.\left.+3 f_{3}^{(1)}(p)+\frac{3}{2} p \frac{\partial f_{3}^{(1)}(p)}{\partial p}\right)\right]\right\} .
\end{aligned}
$$

In $c^{(2)}\left(\mathbf{p}, \mathbf{p}^{\prime}\right)$, there is a second term

$$
\begin{aligned}
c_{\Delta v(I I)}^{(2)}= & -2 \cos \theta(1-\cos \theta)\left(f^{(1)}\left(\mathbf{p}^{\prime}\right)-f^{(1)}(\mathbf{p})\right) \\
& \times \delta\left(p-p^{\prime}\right)\left(\mathbf{n}+\mathbf{n}^{\prime}\right) \cdot \mathbf{v},
\end{aligned}
$$

whose contribution to the collision term is

$$
\begin{aligned}
\frac{1}{2} C_{\Delta v(I I)}^{(2)}(\mathbf{p})= & -\frac{3 n_{e} \sigma_{T} v}{2 p} \int d p^{\prime} p^{\prime} \delta\left(p-p^{\prime}\right) \\
& \times \int_{-1}^{1} \frac{d \mu^{\prime}}{2}\left(f^{(1)}\left(\mathbf{p}^{\prime}\right)-f^{(1)}(\mathbf{p})\right)\left(\mu+\mu^{\prime}\right) \\
& \times \int_{0}^{2 \pi} \frac{d \phi^{\prime}}{2 \pi} \cos \theta(1-\cos \theta) .
\end{aligned}
$$

This integration proceeds through the same steps as for $C_{\Delta v(I)}^{(2)}(\mathbf{p})$. In particular, by noting that $\cos \theta(1-\cos \theta)=$ $-1 / 3+P_{1}(\cos \theta)-2 P_{3}(\cos \theta) / 3$, Equations (105) and (106) allows to compute

$$
\begin{aligned}
& \int \frac{d \phi^{\prime}}{2 \pi} \cos \theta(1-\cos \theta) \\
& \quad=-\frac{1}{3}+P_{1}(\mu) P_{1}\left(\mu^{\prime}\right)-\frac{2}{3} P_{2}(\mu) P_{2}\left(\mu^{\prime}\right),
\end{aligned}
$$

and using the decomposition (96), we arrive at

$$
\begin{aligned}
& \frac{1}{2} C_{\Delta v(I I)}^{(2)}(\mathbf{p}) \\
& =-n_{e} \sigma_{T}\left\{\mathbf{v} \cdot \mathbf{n} f_{2}^{(1)}(p)\left(1-P_{2}(\hat{\mathbf{v}} \cdot \mathbf{n})\right)\right. \\
& \left.\quad+v\left[\frac{1}{5} P_{2}(\hat{\mathbf{v}} \cdot \mathbf{n})\left(3 f_{1}^{(1)}(p)-3 f_{3}^{(1)}(p)\right)\right]\right\} .
\end{aligned}
$$

We then obtain

$$
\begin{aligned}
\frac{1}{2} C_{\Delta v}^{(2)}(\mathbf{p}) & \\
=n_{e} \sigma_{T}\{\mathbf{v} \cdot \mathbf{n}[ & {\left[f^{(1)}(\mathbf{p})-f_{0}^{(1)}(p)-p \frac{\partial f_{0}^{(1)}(p)}{\partial p}-f_{2}^{(1)}(p)\right.} \\
& \left.+\frac{1}{2} P_{2}(\hat{v} \cdot \mathbf{n})\left(f_{2}^{(1)}(p)-p \frac{\partial f_{2}^{(1)}(p)}{\partial p}\right)\right] \\
+v & {\left[2 f_{1}^{(1)}(p)+p \frac{\partial f_{1}^{(1)}(p)}{\partial p}+\frac{1}{5} P_{2}(\hat{v} \cdot \mathbf{n})\right.} \\
& -f_{1}^{(1)}(p)+p \frac{\partial f_{1}^{(1)}(p)}{\partial p} \\
& \left.\left.+6 f_{3}^{(1)}(p)+\frac{3}{2} p \frac{\partial f_{3}^{(1)}(p)}{\partial p}\right]\right\} .
\end{aligned}
$$

As far as the remaining terms, these have already been computed in [64] (see also [65]) and here we just report them (d) $c_{v v}^{(2)}\left(\mathbf{p}, \mathbf{p}^{\prime}\right)$ :

the term proportional to the velocity squared yield a contribution to the collision term

$$
\begin{array}{r}
\frac{1}{2} C_{v v}^{(2)}(\mathbf{p})=n_{e} \sigma_{T}\left\{(\mathbf{v} \cdot \mathbf{n})^{2}\left[p \frac{\partial f^{(0)}}{\partial p}+\frac{11}{20} p^{2} \frac{\partial^{2} f^{(0)}}{\partial p^{2}}\right]\right. \\
\left.+v^{2}\left[p \frac{\partial f^{(0)}}{\partial p}+\frac{3}{20} p^{2} \frac{\partial^{2} f^{(0)}}{\partial p^{2}}\right]\right\} .
\end{array}
$$

(e) $c_{K}^{(2)}\left(\mathbf{p}, \mathbf{p}^{\prime}\right)$ :

The terms responsible for the spectral distortions give

$$
\frac{1}{2} C_{K}^{(2)}(\mathbf{p})=\frac{1}{m_{e} p^{2}} \frac{\partial}{\partial p}\left\{p^{4}\left[T_{e} \frac{\partial f^{(0)}}{\partial p}+f^{(0)}\left(1+f^{(0)}\right)\right]\right\} .
$$

Finally, we write also the part of the collision term coming from (94)

$$
\begin{aligned}
& \delta_{e}^{(1)} c^{(1)}\left(\mathbf{p}, \mathbf{p}^{\prime}\right) \\
& \longrightarrow \delta_{e}^{(1)} C^{(1)}(\mathbf{p})=n_{e} \sigma_{T} \delta_{e}^{(1)} \\
& \quad \times\left[f_{0}^{(1)}(p)+\frac{1}{2} f_{2}^{(1)} P_{2}(\hat{v} \cdot \mathbf{n})-f^{(1)}-p \frac{\partial f^{(0)}}{\partial p} \mathbf{v} \cdot \mathbf{n}\right] .
\end{aligned}
$$

6.2.3. Final Expression for the Collision Term. Summing all the terms, we find the final expression for the collision term (87) up to second order

$$
C(\mathbf{p})=C^{(1)}(\mathbf{p})+\frac{1}{2} C^{(2)}(\mathbf{p}),
$$


with

$$
\begin{aligned}
& C^{(1)}(\mathbf{p}) \\
& =n_{e} \sigma_{T}\left[f_{0}^{(1)}(p)+\frac{1}{2} f_{2}^{(1)} P_{2}(\hat{v} \cdot \mathbf{n}) f^{(1)}-p \frac{\partial f^{(0)}}{\partial p} \mathbf{v} \cdot \mathbf{n},\right]
\end{aligned}
$$

$\frac{1}{2} C^{(2)}(\mathbf{p})$

$$
\begin{aligned}
& =n_{e} \sigma_{T}\left\{\frac{1}{2} f_{00}^{(2)}(p)-\frac{1}{4} \sum_{m=-2}^{2} \frac{\sqrt{4 \pi}}{5^{3 / 2}} f_{2 m}^{(2)}(p) Y_{2 m}(\mathbf{n})\right. \\
& -\frac{1}{2} f^{(2)}(\mathbf{p})+\delta_{e}^{(1)}\left[f_{0}^{(1)}(p)+\frac{1}{2} f_{2}^{(1)} P_{2}(\hat{v} \cdot \mathbf{n})\right. \\
& \frac{d f}{d \eta}=\frac{d f^{(1)}}{d \eta}-p \frac{\partial f^{(0)}}{\partial p} \frac{\partial \Phi^{(1)}}{\partial x^{i}} \frac{d x^{i}}{d \eta} \\
& +p \frac{\partial f^{(0)}}{\partial p} \frac{\partial \Psi^{(1)}}{\partial \eta} \text {. }
\end{aligned}
$$$$
\left.-f^{(1)}-p \frac{\partial f^{(0)}}{\partial p} \mathbf{v} \cdot \mathbf{n}\right]
$$$$
-\frac{1}{2} p \frac{\partial f^{(0)}}{\partial p} \mathbf{v}^{(2)} \cdot \mathbf{n}+\mathbf{v} \cdot \mathbf{n}
$$$$
\times\left[f^{(1)}(\mathbf{p})-f_{0}^{(1)}(p)-p \frac{\partial f_{0}^{(1)}(p)}{\partial p}-f_{2}^{(1)}(p)\right.
$$$$
\left.+\frac{1}{2} P_{2}(\widehat{\mathbf{v}} \cdot \mathbf{n})\left(f_{2}^{(1)}(p)-p \frac{\partial f_{2}^{(1)}(p)}{\partial p}\right)\right]
$$$$
+v\left[2 f_{1}^{(1)}(p)+p \frac{\partial f_{1}^{(1)}(p)}{\partial p}+\frac{1}{5} P_{2}(\widehat{\mathbf{v}} \cdot \mathbf{n})\right.
$$$$
\times\left(-f_{1}^{(1)}(p)+p \frac{\partial f_{1}^{(1)}(p)}{\partial p}\right.
$$$$
\left.\left.+6 f_{3}^{(1)}(p)+\frac{3}{2} p \frac{\partial f_{3}^{(1)}(p)}{\partial p}\right)\right]
$$$$
+(\mathbf{v} \cdot \mathbf{n})^{2}\left[p \frac{\partial f^{(0)}}{\partial p}+\frac{11}{20} p^{2} \frac{\partial^{2} f^{(0)}}{\partial p^{2}}\right]
$$$$
+v^{2}\left[p \frac{\partial f^{(0)}}{\partial p}+\frac{3}{20} p^{2} \frac{\partial^{2} f^{(0)}}{\partial p^{2}}\right]
$$$$
\left.+\frac{1}{m_{e} p^{2}} \frac{\partial}{\partial p}\left[p^{4}\left(T_{e} \frac{\partial f^{(0)}}{\partial p}+f^{(0)}\left(1+f^{(0)}\right)\right)\right]\right\} \text {. }
$$

Notice that there is an internal hierarchy, with terms which do not depend on the baryon velocity $\mathbf{v}$, terms proportional to $\mathbf{v} \cdot \mathbf{n}$ and then to $(\mathbf{v} \cdot \mathbf{n})^{2}, v$ and $v^{2}$ (apart from the Kompaneets terms). In particular, notice the term proportional to $\delta_{e}^{(1)} \mathbf{v} \cdot \mathbf{n}$ is the one corresponding to the Vishniac effect. We point out that we have kept all the terms up to second order in the collision term. In [64, 65], many terms coming from $c_{\Delta v}^{(2)}$ have been dropped mainly because these terms are proportional to the photon distribution function $f^{(1)}$ which on very small scales (those of interest for reionization) is suppressed by the diffusion damping. Here, we want to be completely general and we have to keep them.

\subsection{The Brightness Equation}

6.3.1. First Order. The Boltzmann equation for photons is obtained by combining (80) with (122)-(123). At first order, the left-hand side reads

At first order, it is useful to characterize the perturbations to the Bose-Einstein distribution function (78) in terms of a perturbation to the temperature as

$$
f\left(x^{i}, p, n^{i}, \eta\right)=2\left[\exp \left\{\frac{p}{T(\eta)\left(1+\Theta^{(1)}\right)}\right\}-1\right]^{-1} .
$$

Thus, it turns out that

$$
f^{(1)}=-p \frac{\partial f^{(0)}}{\partial p} \Theta^{(1)}
$$

where we have used the fact that $\partial f /\left.\partial \Theta\right|_{\Theta=0}=-p \partial f^{(0)} / \partial p$. In terms of this variable $\Theta^{(1)}$ the linear collision term (122) will now become proportional to $-p \partial f^{(0)} / \partial p$ which contains the only explicit dependence on $p$, and the same happens for the left-hand side, (124). This is telling us that at first order $\Theta^{(1)}$ does not depend on $p$ but only on $x^{i}, n^{i}, \eta, \Theta^{(1)}=$ $\Theta^{(1)}\left(x^{i}, n^{i}, \tau\right)$. This is well known and the physical reason is that at linear order the energy transfer in Compton collisions between photons and electrons is almost elastic, so that no spectral distortions are generated (in the sense that the temperature fluctuations do not depend on $p$ ). Therefore, the Boltzmann equation for $\Theta^{(1)}$ reads

$$
\begin{aligned}
& \frac{\partial \Theta^{(1)}}{\partial \eta}+n^{i} \frac{\partial \Theta^{(1)}}{\partial x^{i}}+\frac{\partial \Phi^{(1)}}{\partial x^{i}} n^{i}-\frac{\partial \Psi^{(1)}}{\partial \eta} \\
& \quad=n_{e} \sigma_{T} a\left[\Theta_{0}^{(1)}+\frac{1}{2} \Theta_{2}^{(1)} P_{2}(\widehat{\mathbf{v}} \cdot \mathbf{n})-\Theta^{(1)}+\mathbf{v} \cdot \mathbf{n}\right]
\end{aligned}
$$

where we made us of $f_{\ell}^{(1)}=-p \partial f^{(0)} / \partial p \Theta_{\ell}^{(1)}$, according to the decomposition of (96), and we have taken the zeroorder expressions for $d x^{i} / d \eta$, dropping the contribution from $d n^{i} / d \eta$ in (64) since it is already first order.

Notice that since $\Theta^{(1)}$ is independent of $p$, it is equivalent to consider the quantity

$$
\Delta^{(1)}\left(x^{i}, n^{i}, \tau\right)=\frac{\int d p p^{3} f^{(1)}}{\int d p p^{3} f^{(0)}}
$$


being $\Delta^{(1)}=4 \Theta^{(1)}$ at this order. The physical meaning of $\Delta^{(1)}$ is that of a fractional energy perturbation (in a given direction). From (80), another way to write an equation for $\Delta^{(1)}$ — the so-called brightness equation - is

$$
\begin{aligned}
\frac{d}{d \eta} & {\left[\Delta^{(1)}+4 \Phi^{(1)}\right]-4 \frac{\partial}{\partial \eta}\left(\Phi^{(1)}+\Psi^{(1)}\right) } \\
& =n_{e} \sigma_{T} a\left[\Delta_{0}^{(1)}+\frac{1}{2} \Delta_{2}^{(1)} P_{2}(\hat{\mathbf{v}} \cdot \mathbf{n})-\Delta^{(1)}+4 \mathbf{v} \cdot \mathbf{n}\right] .
\end{aligned}
$$

6.3.2. Second Order. The previous results show that at linear order the photon distribution function has a Planck spectrum with the temperature that at any point depends on the photon direction. At second order, one could characterize the perturbed photon distribution function in a similar way as in (125)

$$
f\left(x^{i}, p, n^{i}, \eta\right)=2\left[\exp \left\{\frac{p}{T(\eta) e^{\Theta}}-1\right\}\right]^{-1}
$$

where by expanding $\Theta=\Theta^{(1)}+\Theta^{(2)} / 2+\cdots$ as usual one recovers the first-order expression. For example, in terms of $\Theta$, the perturbation of $f^{(1)}$ is given by (126), while at second order

$$
\frac{f^{(2)}}{2}=-\frac{p}{2} \frac{\partial f^{(0)}}{\partial p} \Theta^{(2)}+\frac{1}{2}\left(p^{2} \frac{\partial^{2} f^{(0)}}{\partial p^{2}}+p \frac{\partial f^{(0)}}{\partial p}\right)\left(\Theta^{(1)}\right)^{2} .
$$

However, as discussed in details in $[64,65]$, now the second-order perturbation $\Theta^{(2)}$ will not be momentum independent because the collision term in the equation for $\Theta^{(2)}$ does depend explicitly on $p$ (defining the combination $-\left(p \partial f^{(0)} / \partial p\right)^{-1} f^{(2)}$ does not lead to a secondorder momentum independent equation as above). Such dependence is evident, for example, in the terms of $C^{(2)}(\mathbf{p})$, (123), proportional to $v$ or $v^{2}$, and in the Kompaneets terms. The physical reason is that nonlinearities in the energy exchange between photons and electrons during Compton collisions bring spectral distortions. For example, in the isotropic limit, only the Kompaneets terms survive giving rise to the Sunyaev-Zeldovich distorsions. As discussed in [65], the Sunyaev-Zeldovich distorsions can also be obtained with the correct coefficients by replacing the average over the direction electron $\left\langle v^{2}\right\rangle$ with the mean squared thermal velocity $\left\langle v_{t h}^{2}\right\rangle=3 T_{e} / m_{e}$ in (123). This is due simply to the fact that the distinction between thermal and bulk velocity of the electrons is just for convenience. This fact also shows that spectral distorsions due to the bulk flow (kinetic SunyaevZeldovich) has the same form as the thermal effect. Thus spectral distorsions can be in general described by a global Compton $y$-parameter (see [65] for a full discussion of spectral distorsions and also [66]). However, in the following we will not be interested in the frequency dependence but only in the anisotropies of the radiation distribution.
Therefore we can integrate over the momentum $p$ and define $[64,65]$

$$
\Delta^{(2)}\left(x^{i}, n^{i}, \tau\right)=\frac{\int d p p^{3} f^{(2)}}{\int d p p^{3} f^{(0)}},
$$

as in (128).

Integration over $p$ of (80)-(123) is straightforward using the following relations:

$$
\begin{gathered}
\int d p p^{3} p \frac{\partial f^{(0)}}{\partial p}=-4 N \\
\int d p p^{3} p^{2} \frac{\partial^{2} f^{(0)}}{\partial p^{2}}=20 N \\
\int d p p^{3} f^{(1)}=N \Delta^{(1)} \\
\int d p p^{3} p \frac{\partial f^{(1)}}{\partial p}=-4 N \Delta^{(1)}
\end{gathered}
$$

Here, $N=\int d p p^{3} f^{(0)}$ is the normalization factor (it is just proportional the background energy density of photons $\bar{\rho}_{\gamma}$ ). At first order one recovers (129). At second, order we find

$$
\begin{aligned}
\frac{1}{2} \frac{d}{d \eta}\left[\Delta^{(2)}+\right. & \left.4 \Phi^{(2)}\right]+\frac{d}{d \eta}\left[\Delta^{(1)}+4 \Phi^{(1)}\right] \\
- & 4 \Delta^{(1)}\left(\Psi^{(1)^{\prime}}-\Phi_{, i}^{(1)} n^{i}\right)-2 \frac{\partial}{\partial \eta}\left(\Psi^{(2)}+\Phi^{(2)}\right) \\
+ & 4 \frac{\partial \omega_{i}}{\partial \eta} n^{i}+2 \frac{\partial \chi_{i j}}{\partial \eta} n^{i} n^{j} \\
=-\frac{\tau^{\prime}}{2}[ & \Delta_{00}^{(2)}-\Delta^{(2)}-\frac{1}{2} \sum_{m=-2}^{2} \frac{\sqrt{4 \pi}}{5^{3 / 2}} \Delta_{2 m}^{(2)} \\
& \times Y_{2 m}(\mathbf{n})+2\left(\delta_{e}^{(1)}+\Phi^{(1)}\right) \\
& \times\left(\Delta_{0}^{(1)}+\frac{1}{2} \Delta_{2}^{(1)} P_{2}(\hat{\mathbf{v}} \cdot \mathbf{n})-\Delta^{(1)}+4 \mathbf{v} \cdot \mathbf{n}\right) \\
& +4 \mathbf{v}^{(2)} \cdot \mathbf{n}+2(\mathbf{v} \cdot \mathbf{n}) \\
& \times\left[\Delta^{(1)}+3 \Delta_{0}^{(1)}-\Delta_{2}^{(1)}\left(1-\frac{5}{2} P_{2}(\hat{\mathbf{v}} \cdot \mathbf{n})\right)\right] \\
& \left.-v \Delta_{1}^{(1)}\left(4+2 P_{2}(\hat{\mathbf{v}} \cdot \mathbf{n})\right)+14(\mathbf{v} \cdot \mathbf{n})^{2}-2 v^{2}\right],
\end{aligned}
$$

where we have expanded the angular dependence of $\Delta$ as in (97)

$$
\Delta^{(i)}(\mathbf{x}, \mathbf{n})=\sum_{\ell} \sum_{m=-\ell}^{\ell} \Delta_{\ell m}^{(i)}(\mathbf{x})(-i)^{\ell} \sqrt{\frac{4 \pi}{2 \ell+1}} Y_{\ell m}(\mathbf{n}),
$$


with

$$
\Delta_{\ell m}^{(i)}=(-i)^{-\ell} \sqrt{\frac{2 \ell+1}{4 \pi}} \int d \Omega \Delta^{(i)} Y_{\ell m}^{*}(\mathbf{n})
$$

where we recall that the superscript stands by the order of the perturbation. When going to Fourier space, some convolutions will appear and the coefficients $\Delta_{\ell m}^{(1)}\left(\mathbf{k}^{\prime}, \eta\right)$ are related to the more familiar ones $\Delta_{\ell}^{(1)}\left(\mathbf{k}^{\prime}, \eta\right)$ as

$$
\Delta_{\ell m}^{(1)}\left(\mathbf{k}^{\prime}\right)=i \sqrt{4 \pi(2 \ell+1)} Y_{\ell m}^{*}\left(\hat{\mathbf{k}}^{\prime}\right) \Delta_{\ell}^{(1)}\left(\mathbf{k}^{\prime}\right) .
$$

Equation (137) is obtained for linear perturbations by inserting into (136) the expansion

$$
\Delta^{(1)}(\mathbf{k}, \mathbf{n})=\sum_{l^{\prime}}\left(2 l^{\prime}+1\right) \Delta_{l^{\prime}}^{(1)}(\mathbf{k}) P_{l^{\prime}}(\mu),
$$

and expanding the Legendre polynomials as

$$
P_{l^{\prime}}(\mu)=\frac{4 \pi}{2 l^{\prime}+1} \sum_{m^{\prime}=-l^{\prime}}^{m^{\prime}=l^{\prime}} Y_{l^{\prime} m^{\prime}}(\mathbf{n}) Y_{l^{\prime} m^{\prime}}^{*}(\hat{\mathbf{k}}) .
$$

Notice that the expansion (138) is valid only for linear scalar perturbations, which is consistent with our treatment where at linear order we neglect vector and tensor perturbation modes.

In (134), we have introduced the differential optical depth

$$
\tau^{\prime}=-\bar{n}_{e} \sigma_{T} a .
$$

It is understood that on the left-hand side of (134) one has to pick up for the total time derivatives only those terms which contribute to second order. Thus, we have to take

$$
\begin{aligned}
\frac{1}{2} & \frac{d}{d \eta}\left[\Delta^{(2)}+4 \Phi^{(2)}\right]+\left.\frac{d}{d \eta}\left[\Delta^{(1)}+4 \Phi^{(1)}\right]\right|^{(2)} \\
= & \frac{1}{2}\left(\frac{\partial}{\partial \eta}+n^{i} \frac{\partial}{\partial x^{i}}\right)\left(\Delta^{(2)}+4 \Phi^{(2)}\right)+n^{i}\left(\Phi^{(1)}+\Psi^{(1)}\right) \\
& \times \partial_{i}\left(\Delta^{(1)}+4 \Phi^{(1)}\right)+\left[\left(\Phi_{, j}^{(1)}+\Psi_{, j}^{(1)}\right) n^{i} n^{j}-\left(\Phi^{, i}+\Psi^{, i}\right)\right] \\
& \times \frac{\partial \Delta^{(1)}}{\partial n^{i}},
\end{aligned}
$$

where we used (66) and (77).

6.3.3. Hierarchy Equations for Multipole Moments. Let us now move to Fourier space. In the following, for a given wave-vector $\mathbf{k}$, we will choose the coordinate system such that $\mathbf{e}_{3}=\hat{\mathbf{k}}$ and the polar angle of the photon momentum is $\vartheta$, with $\mu=\cos \vartheta=\hat{\mathbf{k}} \cdot \mathbf{n}$. Then, (134) can be written as

$$
\Delta^{(2)^{\prime}}+i k \mu \Delta^{(2)}-\tau^{\prime} \Delta^{(2)}=S(\mathbf{k}, \mathbf{n}, \eta),
$$

where $S(\mathbf{k}, \mathbf{n}, \eta)$ can be easily read off (134). We now expand the temperature anisotropy in the multipole moments $\Delta_{\ell m}^{(2)}$ in order to obtain a system of coupled differential equations. By applying the angular integral of (136) to (142), we find

$$
\begin{aligned}
\Delta_{\ell m}^{(2)^{\prime}}(\mathbf{k}, \eta)= & k\left[\frac{\kappa_{\ell m}}{2 \ell-1} \Delta_{\ell-1, m}^{(2)}-\frac{\kappa_{\ell+1, m}}{2 \ell+3} \Delta_{\ell+1, \mathrm{~m}}^{(2)}\right] \\
& +\tau^{\prime} \Delta_{\ell m}^{(2)}+S_{\ell m}
\end{aligned}
$$

where the first term on the right-hand side of (143) has been obtained by using the relation

$$
\begin{aligned}
& i \mathbf{k} \cdot \mathbf{n} \Delta^{(2)}(\mathbf{k}) \\
& =\sum_{\ell m} \Delta_{\ell m}^{(2)}(\mathbf{k}) \frac{k}{2 \ell+1}\left[\kappa_{\ell m} \widetilde{G}_{\ell-1, m}-\kappa_{\ell+1, m} \widetilde{G}_{\ell+1, m}\right] \\
& =k \sum_{\ell m}\left[\frac{\kappa_{\ell m}}{2 \ell-1} \Delta_{\ell-1, m}^{(2)}-\frac{\kappa_{\ell m}}{2 \ell+3} \Delta_{\ell+1, m}^{(2)}\right] \widetilde{G}_{\ell m},
\end{aligned}
$$

where $\widetilde{G}_{\ell m}=(-i)^{\ell} \sqrt{4 \pi /(2 \ell+1)} Y_{\ell m}(\mathbf{n})$ is the angular mode for the decomposition (135) and $\kappa_{\ell m}=\sqrt{l^{2}-m^{2}}$. This relation has been discussed in $[67,68]$ and corresponds to the term $n^{i} \partial \Delta^{(2)} / \partial x^{i}$ in (134). In (143) $S_{l m}$, are the multipole moments of the source term according to the decomposition (136). We do not show its complete expression here, since it is very long, and we prefer to make some general comments (for some specific examples about the terms $S_{l m}$ see Section 10). As it should be already clear from the calculations involving second-order perturbations performed so far, (143) has the same functional form as the Boltzmann equation at linear order (just replace the order of the linear perturbations (1) with that of intrinsically second-order terms (2) with the exception of the source terms $S_{l m}$ which now contain both intrinsic second-order perturbations and also products of first-order perturbations. Therefore, as expected, at second order we recover some intrinsic effects which are characteristic of the linear regime. In (143), (144) represents the free streaming effect: when the radiation undergoes free-streaming, the inhomogeneities of the photon distribution are seen by the obsever as angular anisotropies. At first order, it is responsible for the hierarchy of Boltzmann equations coupling the different $\ell$ modes, and it represents a projection effect of fluctuations on a scale $k$ onto the angular scale $\ell \sim k \eta$. The term $\tau^{\prime} \Delta_{\ell m}^{(2)}$ causes an exponential suppression of anisotropies in the absence of the source term $S_{\ell m}$. The source term contains additional scattering processes and gravitational effects. The intrinsically second-order part of the source term just reproduces the expression of the first order case. Of course the dynamics of the second-order metric and baryon-velocity perturbations which appear will be different and governed by the second-order Einstein equations and continuity equations. The remaining terms in the source are second-order effects generated as nonlinear combinations of the primordial (first-order) perturbations. Notice in particular that they involve the first-order anisotropies $\Delta_{\ell}^{(1)}$ and as a consequence such terms contribute to generate the hierarchy of equations (apart from the free-streaming effect). On large scales (above the horizon at recombination), we can say that the main effects are due to gravity, and they include 
the Sachs-Wolfe and the (late and early) Sachs-Wolfe effect due to the redshift photons suffer when travelling through the second-order gravitational potentials. These, together with the contribution due to the second-order tensor modes, have been already studied in details in [31] (see also [37]). Another important gravitational effect is that of lensing of photons as they travel from the last scattering surface to us. A contribution of this type is given by the last term of (141). On the other hand, examples of second-order scattering effects are the terms proportional to the square of the baryonvelocity fluid $\left(v^{2}\right)$, giving rise to the quadratic Doppler effect (like those in the last line of (134)), or those coupling the photon fluctuations to the baryon velocity (second-line from the bottom of (134)). The Vishniac effect corresponds to the terms proportional to $\delta_{e}^{(1)}$. Finally, notice that in the Boltzmann equation (134)) the second-order baryon velocity appears. At linear order, the baryon velocity is irrotational, meaning that it is the gradient of a potential, and thus in Fourier space it is parallel to $\hat{\mathbf{k}}$, and following the same notation of [69], we write

$$
\mathbf{v}^{(1)}(\mathbf{k})=-i v_{0}^{(1)}(\mathbf{k}) \hat{\mathbf{k}}
$$

The second-order velocity perturbation will contain a transverse (divergence-free) part whose components are orthogonal to $\hat{\mathbf{k}}=\mathbf{e}_{3}$, and we can write

$$
\mathbf{v}^{(2)}(\mathbf{k})=-i v_{0}^{(2)}(\mathbf{k}) \mathbf{e}_{3}+\sum_{m= \pm 1} v_{m}^{(2)} \frac{\mathbf{e}_{2} \mp i \mathbf{e}_{1}}{\sqrt{2}}
$$

where $\mathbf{e}_{i}$ form an orthonormal basis with $\hat{\mathbf{k}}$. The secondorder perturbation $\omega_{i}$ is decomposed in a similar way, with $\omega_{ \pm 1}$ the corresponding components (in this case, in the Poisson gauge, there is no scalar component). Similarly for the tensor perturbation $\chi_{i j}$ we have indicated its amplitudes as $\chi_{ \pm 2}$ in the decomposition [68]

$$
\chi_{i j}=\sum_{m= \pm 2}-\sqrt{\frac{3}{8}} \chi_{m}\left(\mathbf{e}_{1} \pm i \mathbf{e}_{2}\right)_{i}\left(\mathbf{e}_{1} \pm i \mathbf{e}_{2}\right)_{j} .
$$

In computing the source term $S_{l m}$, one has to take into account that in the gravitational part of the Boltzmann equation and in the collision term there are some terms, like $\delta_{e}^{(1)} \mathbf{v}$, which still can be decomposed in the scalar and transverse parts in Fourier space as in (146). For a generic quantity $f(\mathbf{x}) \mathbf{v}$, one can indicate the corresponding scalar and vortical components with $(f \mathbf{v})_{m}$ and their explicit expression is easily found by projecting the Fourier modes of $f(\mathbf{x}) \mathbf{v}$ along the $\hat{\mathbf{k}}=\mathbf{e}_{3}$ and $\left(\mathbf{e}_{2} \mp i \mathbf{e}_{1}\right)$ directions

$$
(f \mathbf{v})_{m}(\mathbf{k})=\int \frac{d^{3} k_{1}}{(2 \pi)^{3}} v_{0}^{(1)}\left(\mathbf{k}_{1}\right) f\left(\mathbf{k}_{2}\right) Y_{1 m}^{*}\left(\hat{k}_{1}\right) \sqrt{\frac{4 \pi}{3}} .
$$

Similarly, for a term like $f(\mathbf{x}) \nabla g(\mathbf{x})$ one can use the notation

$$
(f \nabla g)_{m}(\mathbf{k})=-\int \frac{d^{3} k_{1}}{(2 \pi)^{3}} k_{1} g\left(\mathbf{k}_{1}\right) f\left(\mathbf{k}_{2}\right) Y_{1 m}^{*}\left(\hat{\mathbf{k}}_{1}\right) \sqrt{\frac{4 \pi}{3}} .
$$

6.3.4. Integral Solution of the Second-Order Boltzmann Equation. As in linear theory, one can derive an integral solution of the Boltzmann equation (134) in terms of the source term $S$. Following the standard procedure (see, e.g., [5]) for linear perturbations, we write the left-hand side as $\Delta^{(2)^{\prime}}+$ $i k \mu \Delta^{(2)}-\tau^{\prime} \Delta^{(2)}=e^{-i k \mu \eta+\tau} d\left[\Delta^{(2)} e^{i k \mu \eta-\tau}\right] / d \eta$ in order to derive the integral solution

$$
\Delta^{(2)}\left(\mathbf{k}, \mathbf{n}, \eta_{0}\right)=\int_{0}^{\eta_{0}} d \eta S(\mathbf{k}, \mathbf{n}, \eta) e^{i k \mu\left(\eta-\eta_{0}\right)-\tau}
$$

where $\eta_{0}$ stands by the present time. The expression of the photon moments $\Delta_{\ell m}^{(2)}$ can be obtained as usual from (136). In the previous section we have already found the coefficients for the decomposition of source term $S$

$$
S(\mathbf{k}, \mathbf{n}, \eta)=\sum_{\ell} \sum_{m=-\ell}^{\ell} S_{\ell m}(\mathbf{k}, \eta)(-i)^{\ell} \sqrt{\frac{4 \pi}{2 \ell+1}} Y_{\ell m}(\mathbf{n}) .
$$

In (150), there is an additional angular dependence in the exponential. It is easy to take it into account by recalling that

$$
e^{i \mathbf{k} \cdot \mathbf{x}}=\sum_{\ell}(i)^{\ell}(2 \ell+1) j_{\ell}(k x) P_{\ell}(\hat{\mathbf{k}} \cdot \hat{\mathbf{x}}) .
$$

Thus the angular integral (136) is computed by using the decomposition of the source term (151) and (152)

$$
\begin{aligned}
\Delta_{\ell m}^{(2)}\left(\mathbf{k}, \eta_{0}\right)= & (-1)^{-m}(-i)^{-\ell}(2 \ell+1) \int_{0}^{\eta_{0}} d \eta e^{-\tau(\eta)} \\
& \times \sum_{\ell_{2}} \sum_{m_{2}=-\ell_{2}}^{\ell_{2}}(-i)^{\ell_{2}} S_{\ell_{2} m_{2}} \sum_{\ell_{1}} i^{\ell_{1}} j_{\ell_{1}}\left(k\left(\eta-\eta_{0}\right)\right) \\
& \times\left(2 \ell_{1}+1\right)\left(\begin{array}{ccc}
\ell_{1} & \ell_{2} & \ell \\
0 & 0 & 0
\end{array}\right)\left(\begin{array}{ccc}
\ell_{1} & \ell_{2} & \ell \\
0 & m_{2} & -m
\end{array}\right),
\end{aligned}
$$

where the Wigner 3-j symbols appear because of the Gaunt integrals

$$
\begin{aligned}
g_{\ell_{1} \ell_{2} \ell_{3}}^{m_{1} m_{3} m_{3}} \equiv & \int d^{2} \hat{\mathbf{n}} Y_{\ell_{1} m_{1}}(\hat{\mathbf{n}}) Y_{\ell_{2} m_{2}}(\hat{\mathbf{n}}) Y_{\ell_{3} m_{3}}(\widehat{\mathbf{n}}) \\
= & \sqrt{\frac{\left(2 \ell_{1}+1\right)\left(2 \ell_{2}+1\right)\left(2 \ell_{3}+1\right)}{4 \pi}} \\
& \times\left(\begin{array}{ccc}
\ell_{1} & \ell_{2} & \ell_{3} \\
0 & 0 & 0
\end{array}\right)\left(\begin{array}{ccc}
\ell_{1} & \ell_{2} & \ell_{3} \\
m_{1} & m_{2} & m_{3}
\end{array}\right),
\end{aligned}
$$


Since the second of the Wigner $3-j$ symbols in (153) is nonzero only if $m=m_{2}$, our solution can be rewritten to recover the corresponding expression found for linear anisotropies in [68]

$$
\frac{\Delta_{\ell m}^{(2)}\left(\mathbf{k}, \eta_{0}\right)}{2 \ell+1}=\int_{0}^{\eta_{0}} d \eta e^{-\tau(\eta)} \sum_{\ell_{2}} S_{\ell_{2} m} j_{\ell}^{\left(\ell_{2}, m\right)}\left[k\left(\eta_{0}-\eta\right)\right]
$$

where $j_{\ell}^{\left(\ell_{2}, m\right)}\left[k\left(\eta_{0}-\eta\right)\right]$ are the so called radial functions. Of course the main information at second order is included in the source term containing different effects due to the nonlinearity of the perturbations. In the total angular momentum method of [68], (155) is interpreted just as the intergration over the radial coordinate $\left(\chi=\eta_{0}-\eta\right)$ of the projected source term. Another important comment is that, as in linear theory, the integral solution (153) is in fact just a formal solution, since the source term $S$ contains itself the (intrinsically) second-order photon moments up to $l=2$ (and the products of first-order perturbations in principle may contain $l$-modes up to infinity). This means that one has anyway to resort to the hierarchy equations for photons, (143), to solve for these moments. Once the moments entering in the source function are computed the higher moments are obtained from the integral solution.

6.4. The Boltzmann Equation for Baryons and Cold Dark Matter. In this section we will derive the Boltzmann equation for massive particles, which is the case of interest for baryons and dark matter. These equations are necessary to find the time evolution of number densities and velocities of the baryon fluid which appear in the brightness equation, thus allowing to close the system of equations. Let us start from the baryon component. Electrons are tightly coupled to protons via the Coulomb interactions. This forces the relative energy density contrasts and the velocities to a common value, $\delta_{e}=\delta_{p} \equiv \delta_{b}$ and $v_{e}=v_{p} \equiv v$, so that we can identify electrons and protons collectively as "baryonic" matter.

To derive the Boltzmann equation for baryons let us first focus on the collisionless equation and compute therefore $d g / d \eta$, where $g$ is the distribution function for a massive species with mass $m$. One of the differences with respect to photons is just that baryons are nonrelativistic for the epochs of interest. Thus, the first step is to generalize the formulae in Section 4 up to (77) to the case of a massive particle. In this case, one enforces the constraint $Q^{2}=g_{\mu \nu} Q^{\mu} Q^{\nu}=-m^{2}$ and it also useful to use the particle energy $E=\sqrt{q^{2}+m^{2}}$, where $q$ is defined as in (59). Moreover, in this case it is very convenient to take the distribution function as a function of the variables $q^{i}=q n^{i}$, the position $x^{i}$ and time $\eta$, without using the explicit splitting into the magnitude of the momentum $q$ (or the energy $E$ ) and its direction $n^{i}$. Thus, the total time derivative of the distribution functions reads

$$
\frac{d g}{d \eta}=\frac{\partial g}{\partial \eta}+\frac{\partial g}{\partial x^{i}} \frac{d x^{i}}{d \eta}+\frac{\partial g}{\partial q^{i}} \frac{d q^{i}}{d \eta}
$$

We will not give the details of the calculation since we just need to replicate the same computation we did for the photons. For the four momenta of the particle, notice that $Q^{i}$ has the same form as (62), while for $Q^{0}$, we find

$$
Q^{0}=\frac{e^{-\Phi}}{a} E\left(1+\omega_{i} \frac{q^{i}}{E}\right)
$$

In the following, we give the expressions for $d x^{i} / d \eta$ and $d q^{i} / d \eta$.

(a) As in (66) $d x^{i} / d \eta=Q^{i} / Q^{0}$ and it turns out to be

$$
\frac{d x^{i}}{d \eta}=\frac{q}{E} n^{i} e^{\Phi+\Psi}\left(1-\omega_{i} n^{i} \frac{q}{E}\right)\left(1-\frac{1}{2} \chi_{k m} n^{k} n^{m}\right) .
$$

(b) For $d q^{i} / d \eta$ we need the expression of $Q^{i}$ which is the same as that of $(62)$

$$
Q^{i}=\frac{q^{i}}{a} e^{\Psi}\left(1-\frac{1}{2} \chi_{k m} n^{k} n^{m}\right)
$$

The spatial component of the geodesic equation, up to second order, reads

$$
\begin{aligned}
\frac{d Q^{i}}{d \eta}= & -2\left(\mathcal{H}-\Psi^{\prime}\right)\left(1-\frac{1}{2} \chi_{k m} n^{k} n^{m}\right) \frac{q}{a} n^{i} e^{\Psi}+e^{\Phi+2 \Psi} \\
& \times\left(\frac{\partial \Psi}{\partial x^{k}} \frac{q^{2}}{a E}\left(2 n^{i} n^{k}-\delta^{i k}\right)-\frac{\partial \Phi}{\partial x^{i}} \frac{E}{a}\right) \\
& -\frac{E}{a}\left[\omega^{i^{\prime}}+\mathscr{H} \omega^{i}+q^{k}\left(\chi_{k}^{i^{\prime}}+\omega_{{ }^{\prime} k}^{i}-\omega_{k}^{i^{i}}\right)\right] \\
& +\left[\mathcal{H} \omega^{i} \delta_{j k}-\frac{1}{2}\left(\chi_{j, k}^{i}+\chi_{k, j}^{i}-\chi_{j k}^{i}\right)\right] \frac{q^{j} q^{k}}{E a} .
\end{aligned}
$$

Proceeding as in the massless case, we now take the total time derivative of (159) and using (160), we find

$$
\begin{aligned}
\frac{d q^{i}}{d \eta}= & -\left(\mathscr{H}-\Psi^{\prime}\right) q^{i}+\Psi_{, k} \frac{q^{i} q^{k}}{E} e^{\Phi+\Psi}-\Phi^{, i} E e^{\Phi+\Psi} \\
& -\Psi_{, i} \frac{q^{2}}{E} e^{\Phi+\Psi}-E\left(\omega^{i^{\prime}}+\mathscr{H} \omega^{i}\right)-\left(\chi_{k}^{i^{\prime}}+\omega^{i} k-\omega_{k}^{, i}\right) E q^{k} \\
& +\left[\mathcal{H} \omega^{i} \delta_{j k}-\frac{1}{2}\left(\chi_{j, k}^{i}+\chi_{k, j}^{i}-\chi_{j k}^{i}\right)\right] \frac{q^{j} q^{k}}{E} .
\end{aligned}
$$


We can now write the total time derivative of the distribution function as

$$
\begin{aligned}
& \frac{d g}{d \eta} \\
& =\frac{\partial g}{\partial \eta}+\frac{q}{E} n^{i} e^{\Phi+\Psi}\left(1-\omega_{i} n^{i}-\frac{1}{2} \chi_{k m} n^{k} n^{m}\right) \frac{\partial g}{\partial x^{i}} \\
& +\left[-\left(\mathscr{H}-\Psi^{\prime}\right) q^{i}+\Psi_{, k} \frac{q^{i} q^{k}}{E} e^{\Phi+\Psi}-\Phi^{, i} E e^{\Phi+\Psi}\right. \\
& \quad-\Psi_{, i} \frac{q^{2}}{E} e^{\Phi+\Psi}-E\left(\omega^{i^{\prime}}+\mathscr{H}^{i}\right)-\left(\chi_{k}^{i^{\prime}}+\omega_{\prime k}^{i}-\omega_{k}^{i}\right) E q^{k} \\
& \left.+\left(\mathscr{H}^{i} \delta_{j k}-\frac{1}{2}\left(\chi_{j, k}^{i}+\chi_{k, j}^{i}-\chi_{j k}^{i}\right)\right) \frac{q^{j} q^{k}}{E}\right] \frac{\partial g}{\partial q^{i}} .
\end{aligned}
$$

This equation is completely general since we have just solved for the kinematics of massive particles. As far as the collision terms are concerned, for the system of electrons and protons, we consider the Coulomb scattering processes between the electrons and protons and the Compton scatterings between photons and electrons

$$
\begin{aligned}
\frac{d g_{e}}{d \eta}(\mathbf{x}, \mathbf{q}, \eta) & =\left\langle c_{e p}\right\rangle_{Q Q^{\prime} q^{\prime}}+\left\langle c_{e \gamma}\right\rangle_{p p^{\prime} q^{\prime}}, \\
\frac{d g_{p}}{d \eta}(\mathbf{x}, \mathbf{Q}, \eta) & =\left\langle c_{e p}\right\rangle_{q q^{\prime} Q^{\prime}},
\end{aligned}
$$

where we have adopted the same formalism of [70] with $\mathbf{p}$ and $\mathbf{p}^{\prime}$ the initial and final momenta of the photons, $\mathbf{q}$ and $\mathbf{q}^{\prime}$ the corresponding quantities for the electrons and for protons $\mathbf{Q}$ and $\mathbf{Q}^{\prime}$. The integral over different momenta is indicated by

$$
\langle\cdots\rangle_{p p^{\prime} q^{\prime}} \equiv \int \frac{d^{3} p}{(2 \pi)^{3}} \int \frac{d^{3} p^{\prime}}{(2 \pi)^{3}} \int \frac{d^{3} q^{\prime}}{(2 \pi)^{3}} \ldots,
$$

and thus one can read $c_{e y}$ as the unintegrated part of (82), and similarly for $c_{e p}$ (with the appropriate amplitude $|M|^{2}$ ). In (163) Compton scatterings between protons and photons can be safely neglected because the amplitude of this process has a much smaller amplitude than Compton scatterings with electrons being weighted by the inverse squared mass of the particles.

At this point for the photons we considered the perturbations around the zero-order Bose-Einstein distribution function (which are the unknown quantities). For the electrons (and protons) we can take the thermal distribution described by (84). Moreover, we will take the moments of (163)-(164) in order to find the energy-momentum continuity equations.
6.4.1. Energy Continuity Equations. We now integrate (162) over $d^{3} q /(2 \pi)^{3}$. Let us recall that in terms of the distribution function the number density $n_{e}$, and the bulk velocity $\mathbf{v}$ are given by

$$
\begin{gathered}
n_{e}=\int \frac{d^{3} q}{(2 \pi)^{3}} g, \\
v^{i}=\frac{1}{n_{e}} \int \frac{d^{3} q}{(2 \pi)^{3}} g \frac{q n^{i}}{E},
\end{gathered}
$$

where one can set $E \simeq m_{e}$ since we are considering nonrelativistic particles. We will also make use of the following relations when integrating over the solid angle $d \Omega$

$$
\int d \Omega n^{i}=\int d \Omega n^{i} n^{j} n^{k}=0, \quad \int \frac{d \Omega}{4 \pi} n^{i} n^{j}=\frac{1}{3} \delta^{i j} .
$$

Finally, notice that $d E / d q=q / E$ and $\partial g / \partial q=(q / E) \partial g / \partial E$.

Thus, the first two integrals just brings $n_{e}^{\prime}$ and $\left(n_{e} v^{i}\right)_{, i}$. Notice that all the terms proportional to the secondorder vector and tensor perturbations of the metric give a vanishing contribution at second order since in this case, we can take the zero-order distribution functions which depends only on $\eta$ and $E$, integrate over the direction and use the fact that $\delta^{i j} \chi_{i j}=0$. The trick to solve the remaining integrals is an integration by parts over $q^{i}$. We have an integral like (the one multiplying $\left.\left(\Psi^{\prime}-\mathscr{H}\right)\right)$

$$
\int \frac{d^{3} q}{(2 \pi)^{3}} q^{i} \frac{\partial g}{\partial q^{i}}=-3 \int \frac{d^{3} q}{(2 \pi)^{3}} g=-3 n_{e},
$$

after an integration by parts over $q^{i}$. The remaining integrals can be solved still by integrating by parts over $q^{i}$. The integral proportional to $\Phi^{, i}$ in (162) gives

$$
\int \frac{d^{3} q}{(2 \pi)^{3}} E=-v_{i} n_{e}
$$

where we have used the fact that $d E / d q^{i}=q^{i} / E$. For the integral

$$
\int \frac{d^{3} q}{(2 \pi)^{3}} \frac{q^{i} q^{k}}{E} \frac{\partial g}{\partial q^{i}}
$$

the integration by parts brings two pieces, one from the derivation of $q^{i} q^{k}$ and one from the derivation of the energy E

$$
\begin{array}{r}
-4 \int \frac{d^{3} q}{(2 \pi)^{3}} g \frac{q^{k}}{E}+\int \frac{d^{3} q}{(2 \pi)^{3}} g \frac{q^{2}}{E} \frac{q^{k}}{E} \\
=-4 v^{k} n_{e}+\int \frac{d^{3} q}{(2 \pi)^{3}} g \frac{q^{2}}{E^{2}} \frac{q^{k}}{E} .
\end{array}
$$

The last integral in (172) can indeed be neglected. To check this one makes use of the explicit expression (84) for the distribution function $g$ to derive

$$
\begin{gathered}
\frac{\partial g}{\partial v^{i}}=g \frac{q_{i}}{T_{e}}-\frac{m_{e}}{T_{e}} v_{i} g \\
\int \frac{d^{3} q}{(2 \pi)^{3}} g q^{i} q^{j}=\delta^{i j} n_{e} m_{e} T_{e}+n_{e} m_{e}^{2} v^{i} v^{j} .
\end{gathered}
$$


Thus, it is easy to compute

$$
\frac{\Psi_{, k}}{m_{e}^{3}} \int \frac{d^{3} q}{(2 \pi)^{3}} g q^{2} q^{k}=-\Psi_{, k} v^{2} \frac{T_{e}}{m_{e}}+3 \Psi_{, k} v_{k} n_{e} \frac{T_{e}}{m_{e}}+\Psi_{, k} v_{k} v^{2},
$$

which is negligible taking into account that $T_{e} / m_{e}$ is of the order of the thermal velocity squared.

With these results, we are now able to compute the left-hand side of the Boltzmann equation (163) integrated over $d^{3} q /(2 \pi)^{3}$. The same operation must be done for the collision terms on the right hand side. For example, for the first of the equations in (163) this brings to the integrals $\left\langle c_{e p}\right\rangle_{Q Q^{\prime} q q^{\prime}}+\left\langle c_{e \gamma}\right\rangle_{p p^{\prime} q q^{\prime}}$. However, looking at (83) one realizes that $\left\langle c_{e \gamma}\right\rangle_{p p^{\prime} q q^{\prime}}$ vanishes because the integrand is antisymmetric under the change $\mathbf{q} \leftrightarrow \mathbf{q}^{\prime}$ and $\mathbf{p} \leftrightarrow \mathbf{p}^{\prime}$. In fact this is simply a consequence of the fact that the electron number is conserved for this process. The same argument holds for the other term $\left\langle c_{e p}\right\rangle_{Q Q^{\prime} q q^{\prime}}$. Therefore, the righthand side of (163) integrated over $d^{3} q /(2 \pi)^{3}$ vanishes and we can give the evolution equation for $n_{e}$. Collecting the results of (169) to (175), we find

$$
\begin{aligned}
\frac{\partial n_{e}}{\partial \eta}+ & e^{\Phi+\Psi} \frac{\partial\left(v^{i} n_{e}\right)}{\partial x^{i}}+3\left(\mathscr{H}-\Psi^{\prime}\right) n_{e} \\
& +e^{\Phi+\Psi} v^{k} n_{e}\left(\Phi_{, k}-2 \Psi_{, k}\right)=0 .
\end{aligned}
$$

Similarly, for CDM particles, we find

$$
\begin{gathered}
\frac{\partial n_{\mathrm{CDM}}}{\partial \eta}+e^{\Phi+\Psi} \frac{\partial\left(v^{i} n_{\mathrm{CDM}}\right)}{\partial x^{i}}+3\left(\mathscr{H}-\Psi^{\prime}\right) n_{\mathrm{CDM}} \\
+e^{\Phi+\Psi} v_{\mathrm{CDM}}^{k} n_{\mathrm{CDM}}\left(\Phi_{, k}-2 \Psi_{, k}\right)=0 .
\end{gathered}
$$

6.4.2. Momentum Continuity Equations. Let us now multiply (162) by $\left(q^{i} / E\right) /(2 \pi)^{3}$ and integrate over $d^{3} q$. In this way, we will find the continuity equation for the momentum of baryons. The first term just gives $\left(n_{e} v^{i}\right)^{\prime}$. The second integral is of the type

$$
\frac{\partial}{\partial x^{j}} \int \frac{d^{3} q}{(2 \pi)^{3}} g \frac{q n^{j}}{E} \frac{q n^{i}}{E}=\frac{\partial}{\partial x^{j}}\left(n_{e} \frac{T_{e}}{m_{e}} \delta^{i j}+n_{e} v^{i} v^{j}\right),
$$

where we have used (174) and $E=m_{e}$. The third term proportional to $\left(\mathscr{H}-\Psi^{\prime}\right)$ is

$$
\int \frac{d^{3} q}{(2 \pi)^{3}} q^{k} \frac{\partial g}{\partial q_{k}} \frac{q^{i}}{E}=4 n_{e}+\int \frac{d^{3} q}{(2 \pi)^{3}} g \frac{q^{2}}{E^{2}} \frac{q^{i}}{E},
$$

where we have integrated by parts over $q^{i}$. Notice that the last term in (179) is negligible being the same integral we discussewd above in (175). By the same arguments that lead to neglect the term of (175) it is easy to check that all the remaining integrals proportional to the gravitational potentials are negligible except for

$$
-e^{\Phi+\Psi} \Phi_{, k} \int \frac{d^{3} q}{(2 \pi)^{3}} \frac{\partial g}{\partial q_{k}} q^{i}=n_{e} e^{\Phi+\Psi} \Phi^{, i}
$$

The integrals proportional to the second-order vector and tensor perturbations vanish as vector and tensor perturbations are traceless and divergence-free. The only one which survives is the term proportional to $\omega^{i^{\prime}}+\mathscr{H} \omega^{i}$ in (162).

Therefore, for the integral over $d^{3} q q^{i} / E$ of the left-hand side of the Boltzmann equation (162) for a massive particle with mass $m_{e}\left(m_{p}\right)$ and distribution function (84), we find

$$
\begin{aligned}
& \int \frac{d^{3} q}{(2 \pi)^{3}} \frac{q^{i}}{E} \frac{d g_{e}}{d \eta}=\frac{\partial\left(n_{e} v^{i}\right)}{\partial \eta}+4\left(\mathcal{H}-\Psi^{\prime}\right) n_{e} v^{i}+\Phi^{, i} e^{\Phi+\Psi} n_{e} \\
& +e^{\Phi+\Psi}\left(n_{e} \frac{T_{e}}{m_{e}}\right)^{, i}+e^{\Phi+\Psi} \frac{\partial}{\partial x^{j}}\left(n_{e} v^{j} v^{i}\right)+\frac{\partial \omega^{i}}{\partial \eta} n_{e}+\mathscr{H} \omega^{i} n_{e} .
\end{aligned}
$$

Now, in order to derive the momentum conservation equation for baryons, we take the first moment of both (163) and (164) multiplying them by $\mathbf{q}$ and $\mathbf{Q}$, respectively, and integrating over the momenta. Since previously we integrated the left-hand side of these equations over $d^{3} q q^{i} / E$, we just need to multiply the previous integrals by $m_{e}$ for the electrons and for $m_{p}$ for the protons. Therefore, if we sum the first moment of (163) and (164) the dominant contribution on the left-hand side will be that of the protons

$$
\int \frac{d^{3} Q}{(2 \pi)^{3}} Q^{i} \frac{d g_{p}}{d \eta}=\left\langle c_{e p}\left(q^{i}+Q^{i}\right)\right\rangle_{Q Q^{\prime} q q^{\prime}}+\left\langle c_{e \gamma} q^{i}\right\rangle_{p p^{\prime} q q^{\prime}}
$$

Notice that the integral of the Coulomb collision term $c_{e p}\left(q^{i}+Q^{i}\right)$ over all momenta vanishes simply because of momentum conservation (due to the Dirac function $\delta^{4}(q+$ $\left.\left.Q-q^{\prime}-Q^{\prime}\right)\right)$. As far as the Compton scattering is concerned, we have that, following [70],

$$
\left\langle c_{e \gamma} q^{i}\right\rangle_{p p^{\prime} q q^{\prime}}=-\left\langle c_{e \gamma} p^{i}\right\rangle_{p p^{\prime} q q^{\prime}}
$$

still because of the total momentum conservation. Therefore what we can compute now is the integral over all momenta of $c_{e y} p^{i}$. Notice however that this is equivalent just to multiply the Compton collision term $C(\mathbf{p})$ of $(83)$ by $p^{i}$ and integrate over $d^{3} p /\left(2 \pi^{3}\right)$

$$
\left\langle c_{e \gamma} p^{i}\right\rangle_{p p^{\prime} q q^{\prime}}=a e^{\Phi} \int \frac{d^{3} p}{(2 \pi)^{3}} p^{i} C(\mathbf{p}) .
$$

where $C(\mathbf{p})$ has been already computed in (122) and (123).

We will do the integral (184) in the following. First, let us introduce the definition of the velocity of photons in terms of the distribution function

$$
\left(\rho_{\gamma}+p_{\gamma}\right) v_{\gamma}^{i}=\int \frac{d^{3} p}{(2 \pi)^{3}} f p^{i}
$$

where $p_{\gamma}=\rho_{\gamma} / 3$ is the photon pressure and $\rho_{\gamma}$ the energy density. At first order, we get

$$
\frac{4}{3} v_{\gamma}^{(1) i}=\int \frac{d \Omega}{4 \pi} \Delta^{(1)} n^{i}
$$


where $\Delta$ is the photon distribution anisotropies defined in (132). At second order, we instead find

$$
\frac{4}{3} \frac{v_{\gamma}^{(2) i}}{2}=\frac{1}{2} \int \frac{d \Omega}{4 \pi} \Delta^{(2)} n^{i}-\frac{4}{3} \delta_{\gamma}^{(1)} v_{\gamma}^{(1) i} .
$$

Therefore, the terms in (122) and (123) proportional to $f^{(1)}(\mathbf{p})$ and $f^{(2)}(\mathbf{p})$ will give rise to terms containing the velocity of the photons. On the other hand the terms proportional to $f_{0}^{(1)}(p)$ and $f_{00}^{(2)}(p)$, once integrated, vanish because of the integral over the momentum direction $n^{i}$, $\int d \Omega n^{i}=0$. Also, the integrals involving $P_{2}(\widehat{\mathbf{v}} \cdot \mathbf{n})=[3(\widehat{\mathbf{v}}$. $\left.\mathbf{n})^{2}-1\right] / 2$ in the first line of (122) and (123) vanish since

$$
\int d \Omega P_{2}(\widehat{\mathbf{v}} \cdot \mathbf{n}) n^{i}=\hat{v}^{k} \hat{v}^{j} \int d \Omega n_{k} n_{j} n^{i}=0,
$$

where we are using the relations (168). Similarly, all the terms proportional to $v,(\mathbf{v} \cdot \mathbf{n})^{2}$ and $v^{2}$ do not give any contribution to (184) and, in the second-order collision term, one can check that $\int d \Omega Y_{2}(\mathbf{n}) n^{i}=0$. Then there are terms proportional to $(\mathbf{v} \cdot \mathbf{n}) f^{(0)}(p),(\mathbf{v} \cdot \mathbf{n}) p \partial f^{(0)} / \partial p$ and $(\mathbf{v} \cdot \mathbf{n}) p \partial f_{0}^{(1)} / \partial p$ for which we can use the rules (133) when integrating over $p$ while the integration over the momentum direction is

$$
\int \frac{d \Omega}{4 \pi}(\mathbf{v} \cdot \mathbf{n}) n^{i}=v_{k} \int \frac{d \Omega}{4 \pi} n^{k} n^{i}=\frac{1}{3} v^{i} .
$$

Finally, from the second line of (123), we get three integrals. One is

$$
\int \frac{d^{3} p}{(2 \pi)^{3}} p^{i}(\mathbf{v} \cdot \mathbf{n}) f^{(1)}(\mathbf{p})=\bar{\rho}_{\gamma} \int \frac{d \Omega}{4 \pi} \Delta^{(1)}(\mathbf{v} \cdot \mathbf{n}) n^{i},
$$

where $\bar{\rho}_{\gamma}$ is the background energy density of the photons. The second comes from

$$
\begin{aligned}
& \frac{1}{2} \int \frac{d^{3} p}{(2 \pi)^{3}} p^{i}(\mathbf{v} \cdot \mathbf{n}) P_{2}(\hat{\mathbf{v}} \cdot \mathbf{n})\left(f_{2}^{(1)}(p)-p \frac{\partial f_{2}^{(1)}(p)}{\partial p}\right) \\
& =\frac{5}{4} \bar{\rho}_{y} \Delta_{2}^{(1)}\left[3 v_{j} \hat{v}_{k} \hat{v}_{l} \int \frac{d \Omega}{4 \pi} n^{i} n^{j} n^{k} n^{l}-v_{j} \int \frac{d \Omega}{4 \pi} n^{i} n^{j}\right] \\
& \quad=\frac{1}{3} \bar{\rho}_{\gamma} \Delta_{2}^{(1)} \hat{v}^{i},
\end{aligned}
$$

where we have used the rules (133), Equation (168) and $\int(d \Omega / 4 \pi) n^{i} n^{j} n^{k} n^{l}=\left(\delta^{i j} \delta^{k l}+\delta^{i k} \delta^{l j}+\delta^{i l} \delta^{j k}\right) / 15$. In fact, the third integral

$$
-\int \frac{d^{3} p}{(2 \pi)^{3}} p^{i}(\mathbf{v} \cdot \mathbf{n}) f_{2}^{(1)}(p)
$$

exactly cancels the previous one. Summing the various integrals, we find

$$
\begin{aligned}
\int \frac{d \mathbf{p}}{(2 \pi)^{3}} C(\mathbf{p}) \mathbf{p}=n_{e} \sigma_{T} \bar{\rho}_{\gamma}[ & \frac{4}{3}\left(\mathbf{v}^{(1)}-\mathbf{v}_{\gamma}^{(1)}\right)-\int \frac{d \Omega}{4 \pi} \frac{\Delta^{(2)}}{2} \mathbf{n} \\
& +\frac{4}{3} \frac{\mathbf{v}^{(2)}}{2}+\frac{4}{3} \delta_{e}^{(1)}\left(\mathbf{v}^{(1)}-\mathbf{v}_{\gamma}^{(1)}\right) \\
& \left.+\int \frac{d \Omega}{4 \pi} \Delta^{(1)}(\mathbf{v} \cdot \mathbf{n}) \mathbf{n}+\Delta_{0}^{(1)} \mathbf{v}\right] .
\end{aligned}
$$

Equation (193) can be further simplified. Recalling that $\delta_{\gamma}^{(1)}=\Delta_{0}^{(1)}$, we use (187) and notice that

$$
\int \frac{d \Omega}{4 \pi} \Delta^{(1)}(\mathbf{v} \cdot \mathbf{n}) n^{i}=v_{j}^{(1)} \Pi_{\gamma}^{j i}+\frac{1}{3} v^{i} \Delta_{0}^{(1)},
$$

where the photon quadrupole $\Pi_{\gamma}^{i j}$ is defined as

$$
\Pi_{\gamma}^{i j}=\int \frac{d \Omega}{4 \pi}\left(n^{i} n^{j}-\frac{1}{3} \delta^{i j}\right)\left(\Delta^{(1)}+\frac{\Delta^{(2)}}{2}\right) .
$$

Thus, our final expression for the integrated collision term (184) reads

$$
\begin{aligned}
\int \frac{d^{3} p}{(2 \pi)^{3}} C(\mathbf{p}) p^{i} & \\
=n_{e} \sigma_{T} \bar{\rho}_{\gamma}[ & \frac{4}{3}\left(v^{(1) i}-v_{\gamma}^{(1) i}\right)+\frac{4}{3}\left(\frac{v^{(2) i}}{2}-\frac{v_{\gamma}^{(2) i}}{2}\right) \\
& \left.+\frac{4}{3}\left(\delta_{e}^{(1)}+\Delta_{0}^{(1)}\right)\left(v^{(1) i}-v_{\gamma}^{(1) i}\right)+v_{j}^{(1)} \Pi_{\gamma}^{j i}\right] .
\end{aligned}
$$

We are now able to give the momentum continuity equation for baryons by combining $m_{p} d g_{p} / d \eta$ from (181) with the collision term (184)

$$
\begin{gathered}
\frac{\partial\left(\rho_{b} v^{i}\right)}{\partial \eta}+4\left(\mathscr{H}-\Psi^{\prime}\right) \rho_{b} v^{i}+\Phi^{, i} e^{\Phi+\Psi} \rho_{b}+e^{\Phi+\Psi}\left(\rho_{b} \frac{T_{b}}{m_{p}}\right)^{, i} \\
+e^{\Phi+\Psi} \frac{\partial}{\partial x^{j}}\left(\rho_{b} v^{j} v^{i}\right)+\frac{\partial \omega^{i}}{\partial \eta} \rho_{b}+\mathscr{H} \omega^{i} \rho_{b} \\
=-n_{e} \sigma_{T} a \bar{\rho}_{\gamma}\left[\frac{4}{3}\left(v^{(1) i}-v_{\gamma}^{(1) i}\right)+\frac{4}{3}\left(\frac{v^{(2) i}}{2}-\frac{v_{\gamma}^{(2) i}}{2}\right)\right. \\
\left.+\frac{4}{3}\left(\delta_{b}^{(1)}+\Delta_{0}^{(1)}+\Phi^{(1)}\right)\left(v^{(1) i}-v_{\gamma}^{(1) i}\right)+v_{j}^{(1)} \Pi_{\gamma}^{j i}\right],
\end{gathered}
$$

where $\rho_{b}$ is the baryon energy density and, as we previously explained, we took into account that to a good approximation the electrons do not contribute to the mass of baryons. In the following, we will expand explicitly at first and secondorder (197).

6.4.3. Second-Order Momentum Continuity Equation for Baryons. At first order, we find

$$
\frac{\partial v^{(1) i}}{\partial \eta}+\mathscr{H} v^{(1) i}+\Phi^{(1), i}=\frac{4}{3} \tau^{\prime} \frac{\bar{\rho}_{\gamma}}{\bar{\rho}_{b}}\left(v^{(1) i}-v_{\gamma}^{(1) i}\right) .
$$

At second order, there are various simplifications. In particular, notice that the term on the right-hand side of (197) which is proportional to $\delta_{b}$ vanishes when matched to expansion of the left-hand side by virtue of the first-order 
equation (198). Thus, at the end, we find a very simple equation

$$
\begin{gathered}
\frac{1}{2}\left(\frac{\partial v^{(2) i}}{\partial \eta}+\mathscr{H} v^{(2) i}+2 \frac{\partial \omega^{i}}{\partial \eta}+2 \mathcal{H} \omega_{i}+\Phi^{(2), i}\right)-\frac{\partial \Psi^{(1)}}{\partial \eta} v^{(1) i} \\
+v^{(1) j} \partial_{j} v^{(1) i}+\left(\Phi^{(1)}+\Psi^{(1)}\right) \Phi^{(1), i}+\left(\frac{T_{b}}{m_{p}}\right)^{, i} \\
=\frac{4}{3} \tau^{\prime} \bar{\rho}_{\gamma} \bar{\rho}_{b} \times\left[\left(\frac{v^{(2) i}}{2}-\frac{v_{\gamma}^{(2) i}}{2}\right)+\left(\Delta_{0}^{(1)}+\Phi^{(1)}\right)\right. \\
\left.\times\left(v^{(1) i}-v_{\gamma}^{(1) i}\right)+\frac{3}{4} v_{j}^{(1)} \Pi_{\gamma}^{j i}\right],
\end{gathered}
$$

with $\tau^{\prime}=-\bar{n}_{e} \sigma_{T} a$.

6.4.4. Second-Order Momentum Continuity Equation for $C D M$. Since CDM particles are collisionless, at first order, we find

$$
\frac{\partial v_{\mathrm{CDM}}^{(1) i}}{\partial \eta}+\mathscr{H} v_{\mathrm{CDM}}^{(1) i}+\Phi^{(1), i}=0 .
$$

At second order we find

$$
\begin{gathered}
\frac{1}{2}\left(\frac{\partial v_{\mathrm{CDM}}^{(2) i}}{\partial \eta}+\mathscr{H} v_{\mathrm{CDM}}^{(2) i}+2 \frac{\partial \omega^{i}}{\partial \eta}+2 \mathcal{H} \omega_{i}+\Phi^{(2), i}\right)-\frac{\partial \Psi^{(1)}}{\partial \eta} v_{\mathrm{CDM}}^{(1) i} \\
+v_{\mathrm{CDM}}^{(1) j} \partial_{j} v_{\mathrm{CDM}}^{(1) i}+\left(\Phi^{(1)}+\Psi^{(1)}\right) \Phi^{(1), i}+\left(\frac{T_{\mathrm{CDM}}}{m_{\mathrm{CDM}}}\right)^{, i}=0 .
\end{gathered}
$$

\section{CMB Anisotropies at Second-Order at All Scales: Analytical Approach}

7.1. Towards a Second-Order CMB Radiation Transfer Function. As pointed out in Section 6 various nonprimordial sources of non-Gaussianity for the CMB anisotropies can arise from the nonlinear evolution of the cosmological perturbations, including the Sunyaev-Z'eldovich, ISW (ReesSciama) effects, and the gravitational lensing, with possible correlations between these contributions. Here we will focus on another relevant source of non-Gaussianity: the nonlinear effects operating at the recombination epoch. The dynamics at recombination is quite involved because all the nonlinearities in the evolution of the baryon-photon fluid at recombination and the ones coming from general relativity should be accounted for.

The following section can be considered as an application of the Boltzmann equations found previously. Despite they depend on a high numbers of terms, because of the appearance within second-order perturbation theory of products of fist-oder perturbations, it is remarkable that an analytical study of the complicated recombination dynamics is possible. This allows to account for those effects that at the last scattering surface produce a non-Gaussian contribution to the $\mathrm{CMB}$ anisotropies that add to the primordial one. Such a contribution is so relevant because it represents a major part of the second-order radiation transfer function which must be determined in order to have a complete control of both the primordial and nonprimordial part of NG in the $\mathrm{CMB}$ anisotropies and to gain from the theoretical side the same level of precision that could be reached experimentally in the near future $[4,5,71]$.

In order to achieve this goal, we consider the Boltzmann equations derived in Section 6 at second-order describing the evolution of the photon, baryon and CDM fluids, and we manipulate them further under the assumption of tight coupling beteween photons and baryons. This leads to the generalization at second-order of the equations for the photon energy density and velocity perturbations which govern the acoustic oscillations of the photon-baryon fluid for modes that are inside the horizon at recombination. The evolution is that of a damped harmonic oscillator, with a source term which is given by the gravitational potentials generated by the different species. An interesting result is that, unlike the linear case, at second-order the quadrupole moment of the photons is not suppressed in the tight coupling limit and it must be taken into account.

The analytical solutions for the acoustic oscillations of the photon-baryon fluid at second order are derived adopting some simplifications which are also standard for an analytical treatment of the linear $\mathrm{CMB}$ anisotropies, and which nonetheless allow to catch most of the physics at recombination. One of these simplifications is to study separately two limiting regimes: intermediate scales which enter the horizon in between the equality epoch $\left(\eta_{\mathrm{eq}}\right)$ and the recombination epoch $\left(\eta_{r}\right)$, with $\eta_{r}^{-1} \ll k \ll \eta_{\text {eq }}^{-1}$, and shortwave perturbations, with $k \gg \eta_{\mathrm{eq}}^{-1}$, which enter the horizon before the equality epoch. Here, our main concern is to provide a simple estimate of the quantitative behaviour of the nonlinear evolution taking place at recombination, offering at the same time all the tools for a more accurate computation. We find that the second-order $\mathrm{CMB}$ anisotropies generated on the last scattering surface do not reduce only to the energy density and velocity perturbations of the photons evaluated at recombination, but a number of second-order corrections at last scattering arise from the Boltzmann equations of [46] in the form of products of firstorder perturbations.

We will see that the dynamics at recombination is indeed dominated on small scales by the nonlinear evolution of the second-order gravitational potentials feeded by the cold dark matter density perturbations. The gravitational potentials determine the energy density fluctuations of the photons at recombination and their effects show up in the $\mathrm{CMB}$ anisotropies as

$$
\frac{\Delta T}{T}=\frac{1}{4} \Delta_{00}^{(2)}+\Phi^{(2)},
$$

which is the usual term due to the intrinsic fractional temperature fluctuation $\Delta_{00}^{(2)} / 4$ on the last scattering surface $\left(\Delta_{00}^{(2)}\right.$ is the monopole of the photon distribution function) and the gravitational redshift due to the gravitational potential. However, the analysis of the remaining contributions that 
come in the form of products of first-order perturbations is equally important. The reason is that one of the central quantities we are interested in is the contamination to the primordial non-Gaussianity that is produced by the secondary effects. In that respect, the reasonable question to ask is which kind of primordial non-Gaussianity a given secondary effect can contaminate most, whether it is of the so called "local type" or of the "equilateral type", for example. Therefore, it might well be the case that, even if some secondary effects appear to be the dominant ones, they might give a high contamination to a given type of primordial non-Gaussianity, but a low contamination to a different kind of primordial non-Gaussianity, for which "subdominant" terms, on the other hand, represent the strongest contaminant. This section and the following two provide a clear example: we anticipate here that while the dominant contribution on small scales to the term in (202) will mainly mimic an equilateral type of primordial nonGaussianity, second-order effects that come as products of first-order times first-order perturbations actually mainly contaminate local primordial non-Gaussianity.

Notice that the case $k \gg \eta_{\mathrm{eq}}^{-1}$ has been treated in two steps. First, we just assume a radiation dominated universe, and then we provide a much more complete analysis by solving the evolution of the perturbations from the equality epoch onwards taking into account that the dark matter perturbations around the equality epoch tend to dominate the second-order gravitational potentials. As a byproduct, this last step provides the Meszaros effect at second-order. In deriving the analytical solutions, we have accurately accounted for the initial conditions set on superhorizon scales by the primordial non-Gaussianity. In fact, the primordial contribution is always transferred linearly, while the real new contribution to the radiation transfer function is given by all the additional terms provided in the source functions of the equations. Let us stress here that the analysis of the CMB bispectrum performed so far, as for example in [72], adopt just the linear radiation transfer function (unless the bispectrum originated by specific secondary effects, such as Rees-Sciama or Sunyaev-Zel'dovich effects, are considered, see on this Section 8). The formalism to provide in a systematic way the second-order CMB radiation transfer function will be reviewd in Section 10.

7.2. The Boltzmann Equations in the Tightly Coupled Limit. We now derive the moments of the Boltzmann equations for photons in the limit when the photons are tightly coupled to the the baryons (the electron-proton system) due to Compton scattering. This leads to the governing equations for the acoustic oscillations of the photon-baryon fluid. The well-known computation at linear order is briefly reviewed in (C.1) under some standard simplifying assumptions. Here, we will focus on the derivation of the equations at secondorder in the perturbations, pointing out some interesting differences with respect to the linear case. In particular, note that, while we already know that the L.H.S. of the equations at second-order will have the same form as for the linear case, the source term on the R.H.S. of the moments of the
Boltzmann equations will also consist of first-order squared terms.

7.2.1. Energy Continuity Equation. Let us start by integrating (134) over $d \Omega_{n} / 4 \pi$ to get the evolution equation for the second-order photon energy density perturbations $\Delta_{00}^{(2)}$

$$
\begin{aligned}
\Delta_{00}^{(2)^{\prime}} & +\frac{4}{3} \partial_{i} v_{\gamma}^{(2) i}+\frac{8}{3} \partial_{i}\left(\Delta_{00}^{(1)} v_{\gamma}^{(1) i}\right)-4 \Psi^{(2)^{\prime}}+\frac{8}{3}\left(\Phi^{(1)}+\Psi^{(1)}\right) \\
& \times \partial_{i} v_{\gamma}^{(1) i}+2 \int \frac{d \Omega_{\mathbf{n}}}{4 \pi} \\
& \times\left[\left(\Phi_{, j}^{(1)}+\Psi_{, j}^{(1)}\right) n^{i} n^{j}-\left(\Phi^{, i}+\Psi^{, i}\right)\right] \frac{\partial \Delta^{(1)}}{\partial n^{i}} \\
& -8 \Psi^{(1)^{\prime}} \Delta_{00}^{(1)}+\frac{32}{3} \Phi_{, i}^{(1)} v_{\gamma}^{(1) i}=-\frac{8}{3} \tau^{\prime} v_{i}^{(1)}\left(v^{(1) i}-v_{\gamma}^{(1) i}\right)
\end{aligned}
$$

where we have used the explicit definition for the secondorder velocity of the photons [46]

$$
\frac{4}{3} \frac{v_{\gamma}^{(2) i}}{2}=\frac{1}{2} \int \frac{d \Omega}{4 \pi} \Delta^{(2)} n^{i}-\frac{4}{3} \delta_{\gamma}^{(1)} v_{\gamma}^{(1) i} .
$$

We can now make use of the tight coupling expansion to simplify (203). In the L.H.S., we use $\partial_{i} v_{\gamma}^{(1) i}=\partial_{i} v^{(1) i}=$ $3 \Psi^{(1)^{\prime}}-\delta_{b}^{(1)^{\prime}}=3 \Psi^{(1)^{\prime}}-3 \Delta_{00}^{(1)^{\prime}} / 4$ obtained in the tightly coupled limit from (C.7) and (C.9). On the other hand, in the R.H.S. of (203)

$$
\begin{aligned}
\left(v^{(1) i}-v_{\gamma}^{(1) i}\right) & =\frac{R}{\tau^{\prime}}\left(v_{\gamma}^{(1) i^{\prime}}+\mathcal{H} v_{\gamma}^{(1) i}+\Phi^{(1), i}\right) \\
& =\frac{R}{\tau^{\prime}}\left(\frac{\mathscr{H}}{1+R} v_{\gamma}^{(1) i}-\frac{1}{4} \frac{\Delta_{00}^{(1), i}}{1+R}\right),
\end{aligned}
$$

by using (C.4) and the evolution equation for the photon veleocity (C.5). Here, we introduce the baryon-photon ratio

$$
R=\frac{3}{4} \frac{\rho_{b}}{\rho_{\gamma}} .
$$

We thus arrive at the following equation:

$$
\Delta_{00}^{(2)^{\prime}}+\frac{4}{3} \partial_{i} v_{\gamma}^{(2) i}-4 \Psi^{(2)^{\prime}}=\varsigma_{\Delta},
$$

where the source term is given by

$$
\begin{aligned}
\wp_{\Delta}= & \left(\Delta_{00}^{(1) 2}\right)^{\prime}-2\left(\Phi^{(1)}+\Psi^{(1)}\right)\left(4 \Psi^{(1)^{\prime}}-\Delta_{00}^{(1)^{\prime}}\right) \\
& -\frac{8}{3} v_{\gamma}^{(1) i}\left(\Delta_{00}^{(1)}+4 \Phi^{(1)}\right)_{, i} \\
& +\frac{16}{3}\left(\Phi^{(1)}+\Psi^{(1)}\right)^{, i} v_{i} \\
& -\frac{8}{3} R\left(\frac{\mathscr{H}}{1+R} v_{\gamma}^{(1) 2}-\frac{1}{4} \frac{v_{\gamma i}^{(1)} \Delta_{00}^{(1), i}}{1+R}\right) .
\end{aligned}
$$


Notice that the integral in the second line of (203) has been computed by expanding the linear anisotropies as $\Delta^{(1)}=\sum_{\ell}(2 \ell+1) \Delta_{\ell}^{(1)} P_{\ell}(\mathbf{v} \cdot \mathbf{n})$ and expressing explicitly the dependence on $n^{i}$ as $P_{1}(\mathbf{v} \cdot \mathbf{n})=\hat{v}^{i} n_{i}, P_{2}(\mathbf{v} \cdot \mathbf{n})=(3(\mathbf{v}$. $\left.\mathbf{n})^{2}-1\right) / 2$ and so on. This allows to perform the derivative $\partial \Delta^{(1)} / \partial n^{i}$. It turns out that the term proportional to the first-order quadrupole vanishes, and higher order terms can be neglected because of the tight coupling approximation. Therefore, the only term that is non negligible is the dipole term and using $\Delta_{1}^{(1)}=4 v / 3$ in the tight coupling limit, one obtains that the integral is equal to $(16 / 3)\left(\Phi^{(1)}+\Psi^{(1)}\right)^{, i} v_{i}$.

7.2.2. Velocity Continuity Equation. We now derive the second moment of the Boltzmann equation (134) and then we take its tight coupling limit. The integration of (134) over $d \Omega_{n} n^{i} / 4 \pi$ yields the continuity equation for the photon velocity

$$
\begin{aligned}
\frac{4}{3} \frac{v_{\gamma}^{(2) i^{\prime}}}{2} & +\frac{1}{2} \partial_{j} \Pi_{\gamma}^{(2) j i}+\frac{1}{3} \frac{\Delta_{00}^{(2), i}}{2}+\frac{2}{3} \Phi^{(2), i}+\frac{4}{3} \omega^{i^{\prime}} \\
= & -\frac{4}{3}\left(\Delta_{00}^{(1)} v_{\gamma}^{(1) i}\right)^{\prime}+\frac{16}{3} \Psi^{(1)^{\prime}} v_{\gamma}^{(1) i}-4 \Phi_{, j}^{(1)} \Pi_{\gamma}^{(1) j i} \\
& -\frac{4}{3} \Phi^{(1), i} \Delta_{00}^{(1)}-\left(\Phi^{(1)}+\Psi^{(1)}\right) \partial_{j} \Pi_{\gamma}^{(1) j i} \\
& -\frac{1}{3}\left(\Phi^{(1)}+\Psi^{(1)}\right) \Delta_{00}^{(1), i}+\int \frac{d \Omega_{\mathbf{n}}}{4 \pi} \\
\times & n^{i}\left[\left(\Phi_{, j}^{(1)}+\Psi_{, j}^{(1)}\right) n^{i} n^{j}-\left(\Phi^{, i}+\Psi^{, i}\right)\right] \frac{\partial \Delta^{(1)}}{\partial n^{i}} \\
- & \frac{\tau^{\prime}}{2}\left[\frac{4}{3}\left(v^{(2) i}-v_{\gamma}^{(2) i}\right)+\frac{8}{3}\left(\delta_{b}^{(1)}+\Phi^{(1)}+\Delta_{00}^{(1)}\right)\right. \\
& \left.\times\left(v^{(1) i}-v_{\gamma}^{(1)}\right)+2 v_{j}^{(1)} \Pi_{\gamma}^{(1) j i}\right] \\
& -\frac{4}{3} \Phi^{(1), i}\left(\Phi^{(1)}+\Psi^{(1)}\right) .
\end{aligned}
$$

The difference between the second-order baryon and photon velocities $\left(v^{(2) i}-v_{\gamma}^{(2) i}\right)$ appearing in (209) is obtained from the baryon continuity equation which can be written as (see [46])

$$
\begin{aligned}
v^{(2) i}= & v_{\gamma}^{(2) i}+\frac{R}{\tau^{\prime}}\left[\left(v^{(2) i^{\prime}}+\mathscr{H} v^{(2) i}+2 \omega^{i^{\prime}}+2 \mathcal{H} \omega^{i}+\Phi^{(2), i}\right)\right. \\
& \left.-2 \Psi^{(1)^{\prime}} v^{(1) i} \partial_{i} v^{(1) 2}+2 \Phi^{(1), i}\left(\Phi^{(1)}+\Psi^{(1)}\right)\right] \\
& -\frac{3}{2} v_{j}^{(1)} \Pi_{\gamma}^{(1) j i}-2\left(\Delta_{00}^{(1)}+\Phi^{(1)}\right)\left(v^{(1) i}-v_{\gamma}^{(1) i}\right) .
\end{aligned}
$$

We want now to reduce (209) in the tightly coupled limit. We first insert the expression (210) in (209). Notice that the last three terms in (210) will cancel out. On the other hand, in the tight coupling limit expansion one can set $v^{(1) i}=v_{\gamma}^{(1) i}$ and $v^{(2) i}=v_{\gamma}^{(2) i}$ in the remaining terms on the R.H.S. of (210). Thus, (209) becomes

$$
\begin{aligned}
& \left(v_{\gamma}^{(2) i}+2 \omega^{i}\right)^{\prime}+\mathcal{H} \frac{R}{1+R}\left(v_{\gamma}^{(2) i}+2 \omega^{i}\right)+\frac{1}{4} \frac{\Delta_{00}^{(2), i}}{1+R}+\Phi^{(2), i} \\
& =-\frac{3}{4(1+R)} \partial_{j} \Pi_{\gamma}^{(2) j i}-\frac{2}{1+R}\left(\Delta_{00}^{(1)} v_{\gamma}^{(1) i}\right)^{\prime}+\frac{8}{1+R} \Psi^{(1)^{\prime}} v_{\gamma}^{(1) i} \\
& \quad-\frac{2}{1+R} \Phi^{(1), i} \Delta_{00}^{(1)}-\frac{2}{1+R} \Phi^{(1), i}\left(\Phi^{(1)}+\Psi^{(1)}\right) \\
& \quad+2 \frac{R}{1+R} \Psi^{(1)^{\prime}} v_{\gamma}^{(1) i}-\frac{1}{2(1+R)}\left(\Phi^{(1)}+\Psi^{(1)}\right) \Delta_{00}^{(1), i} \\
& \quad-\frac{R}{1+R} \partial^{i} v_{\gamma}^{(1) 2}-2 \frac{R}{1+R}\left(\Phi^{(1)}+\Psi^{(1)}\right) \Phi^{(1), i} \\
& \quad-\tau^{\prime} \frac{2}{1+R} \delta_{b}^{(1)}\left(v^{(1) i}-v_{\gamma}^{(1) i}\right),
\end{aligned}
$$

where in the tightly coupled limit, we are neglecting the first-order quadrupole and (higher-order moments) of the photon distribution since it is suppressed by $1 / \tau$ with respect to the other terms. Moreover, the integral in the fourth line of (209) has been computed following the same steps used for the analogous integral in (203). In this case, it turns out that the contribution from the first-order dipole term vanishes, while the one from the quadrupole is non zero, but this and higher-order terms can be neglected because of the tight coupling approximation, so that the integral is in fact negligible. Next, for the term like $\tau^{\prime} \delta_{b}^{(1)}\left(v^{(1) i}-v_{\gamma}^{(1) i}\right)$ we employ the relation previously derived in (99) with $\delta_{b}^{(1)}=$ $3 \Delta_{00}^{(1)} / 4$ and we use the first order tight coupling equations (C.1) and (C.5) in order to further simplify (211). We finally obtain

$$
v_{\gamma}^{(2) i^{\prime}}+\mathcal{H} \frac{R}{1+R} v_{\gamma}^{(2) i}+\frac{1}{4} \frac{\Delta_{00}^{(2), i}}{1+R}+\Phi^{(2), i}=8_{V}^{i}
$$

where

$$
\begin{aligned}
\mathcal{f}_{V}^{i}= & -\frac{3}{4(1+R)} \partial_{j} \Pi_{\gamma}^{(2) j i}-2 \omega_{i}^{\prime}-2 \mathcal{H} \frac{R}{1+R} \omega^{i} \\
& +2 \frac{\mathcal{H} R}{(1+R)^{2}} \Delta_{00}^{(1)} v_{\gamma}^{(1) i}+\frac{1}{4(1+R)^{2}}\left(\Delta_{00}^{(1) 2}\right)^{, i} \\
& +\frac{8}{3(1+R)} v_{\gamma}^{(1) i} \partial_{j} v_{\gamma}^{(1) j}+2 \frac{R}{1+R} \Psi^{(1)^{\prime}} v_{\gamma}^{(1) i} \\
& -2\left(\Phi^{(1)}+\Psi^{(1)}\right) \Phi^{(1), i}-\frac{1}{2(1+R)}\left(\Phi^{(1)}+\Psi^{(1)}\right) \Delta_{00}^{(1), i} \\
& -\frac{R}{1+R} \partial^{i} v_{\gamma}^{(1) 2}-\frac{3}{2} \frac{R}{1+R} \Delta_{00}^{(1)}\left(\frac{\mathscr{H}}{1+R} v_{\gamma}^{(1) i}-\frac{1}{4} \frac{\Delta_{00}^{(1), i}}{1+R}\right) .
\end{aligned}
$$


We have spent some time in giving the details of the computation for the photon Boltzmann equations at secondorder in the perturbations. As a summary of the results obtained so far, we refer the reader to (207) and (212) as our master equations which we will solve in the next sections. In particular (212) is the second-order counterpart of (C.5) for the photon velocity in the tight coupling regime. Notice that there are two important differences with respect to the linear case. One is that, in (212), there will be a contribution not only from scalar perturbations but also from vector modes which, at second-order, are inevitably generated as nonlinear combinations of first-order scalar perturbations. In particular, we have included the vector metric perturbations $\omega^{i}$ in the source term. Second, and most important, we have also kept in the source term the secondorder quadrupole of the photon distribution $\Pi_{\gamma}^{(2) i j}$. At linear order we can neglect it together with higher order moments of the photons since they turn out to be suppressed with respect to the first two moments in the tight coupling limit by increasing powers of $1 / \tau$. However, in the next section we will show that at second order this does not hold anymore, as the photon quadrupole is no longer suppressed.

Finally, following the same steps leading to (C.6) at linear order we can derive a similar equation for the second-order photon energy density perturbation $\Delta_{00}^{(2)}$ which now will be characterized by the source terms $\delta_{\Delta}$ and $\delta_{V}^{i}$

$$
\begin{aligned}
\left(\Delta_{00}^{(2) \prime \prime}\right. & \left.-4 \Psi^{(2) \prime \prime}\right)+\mathcal{H} \frac{R}{1+R}\left(\Delta_{00}^{(2)^{\prime}}-4 \Psi^{(2)^{\prime}}\right) \\
& -c_{s}^{2} \nabla^{2}\left(\Delta_{00}^{(2)}-4 \Psi^{(2)}\right) \\
= & \frac{4}{3} \nabla^{2}\left(\Phi^{(2)}+\frac{\Psi^{(2)}}{1+R}\right)+\wp_{\Delta}^{\prime}+\mathcal{H} \frac{R}{1+R} \S_{\Delta}-\frac{4}{3} \partial_{i} \delta_{V}^{i},
\end{aligned}
$$

where we have introduced the photon-baryon fluid sound speed $c_{s}=1 / \sqrt{3(1+R)}$.

7.2.3. Second-Order Quadrupole Moment of the Photons in the Tight Coupling Limit. Let us now consider the quadrupole moment of the photon distribution defined in (195) and show that at second-order it cannot be neglected in the tightly coupled limit, unlike for the linear case. We first integrate the R.H.S. of (134) over $d \Omega_{n}\left(n^{i} n^{j}-\delta^{i j} / 3\right) / 4 \pi$ and then we set it to be vanishing in the limit of tight coupling.

The integration involves various pieces to compute. For clarity we will consider each of them separately. The term $\Delta_{00}^{(2)}$ does not contribute. For the third term we can write, from (135)

$$
\begin{aligned}
& -\sum_{m=-2}^{m=2} \frac{\sqrt{\pi}}{5^{3 / 2}} \Delta_{2 m}^{(2)} Y_{2 m} \\
& =\frac{\Delta^{(2)}}{10}-\frac{1}{10} \sum_{\ell \neq 2} \sum_{m=-\ell}^{\ell} \Delta_{\ell m}^{(2)}(-i)^{\ell} \sqrt{\frac{4 \pi}{2 \ell+1}} Y_{\ell m},
\end{aligned}
$$

so that the integral just brings $\Pi_{\gamma}^{(2) i j} / 10$, since the only contribution in (215) comes from $\Delta^{(2)} / 10$ with all the other terms vanishing. The following nontrivial integral is

$$
\begin{aligned}
& \int \frac{d \Omega}{4 \pi}\left(n^{i} n^{j}-\frac{1}{3} \delta^{i j}\right) \Delta_{2}^{(1)} P_{2}(\hat{\mathbf{v}} \cdot \mathbf{n}) \\
& =\hat{v}_{k} \hat{v}_{l} \int \frac{d \Omega}{4 \pi}\left(n^{i} n^{j}-\frac{1}{3} \delta^{i j}\right) \Delta_{2}^{(1)} \times\left(\frac{3}{2} n^{k} n^{l}-\frac{1}{2}\right) \\
& \quad=\frac{\Delta_{2}^{(1)}}{5}\left(\hat{v}^{i} \hat{v}^{j}-\frac{1}{3} \delta^{i j}\right)
\end{aligned}
$$

where the baryon velocity appearing in $P_{2}(\hat{\mathbf{v}} \cdot \mathbf{n})$ is first order and we make use of the relations (168) together with

$$
\int \frac{d \Omega}{4 \pi} n^{i} n^{j} n^{k} n^{l}=\frac{1}{15}\left(\delta^{i j} \delta^{k l}+\delta^{i k} \delta^{l j}+\delta^{i l} \delta^{j k}\right) .
$$

The integrals of $\delta_{e}^{(1)} \Delta_{0}^{(1)}, \delta_{e}^{(1)}(v \cdot n)$ and $v^{(2)} \cdot n$ vanish and

$$
\begin{aligned}
& v \Delta_{1}^{(1)} \int \frac{d \Omega}{4 \pi} P_{2}(\hat{\mathbf{v}} \cdot \mathbf{n})\left(n^{i} n^{j}-\frac{1}{3} \delta^{i j}\right) \\
& \quad=\frac{1}{5} v \Delta_{1}^{(1)}\left(\hat{v}^{i} \hat{v}^{j}-\frac{1}{3} \delta^{i j}\right)=\frac{4}{15}\left(v^{i} v^{j}-\frac{1}{3} \delta^{i j} v^{2}\right)
\end{aligned}
$$

where in the last step, we take $\Delta_{1}^{(1)}=4 v / 3$ in the tight coupling limit. Similarly, the integral of $14(\mathbf{v} \cdot \mathbf{n})^{2}$ brings

$$
14 v^{k} v^{\ell} \int \frac{d \Omega}{4 \pi} n_{k} n_{\ell}\left(n^{i} n^{j}-\frac{1}{3} \delta^{i j}\right)=\frac{28}{15}\left(v^{i} v^{j}-\frac{1}{3} \delta^{i j} v^{2}\right) .
$$

The integral of $2(\mathbf{v} \cdot \mathbf{n}) \Delta^{(1)}$ can be performed by expanding the linear anisotropies as $\Delta^{(1)}=\sum_{\ell}(2 \ell+1) \Delta_{\ell}^{(1)} P_{\ell}(\hat{\mathbf{v}} \cdot \mathbf{n})$. We thus find

$$
\begin{aligned}
v^{k} \hat{v}^{m} & \int \frac{d \Omega}{4 \pi} n_{k}\left(n^{i} n^{j}-\frac{1}{3} \delta^{i j}\right) n_{m} \Delta_{1}^{(1)}+\mathcal{O}_{\ell>2} \\
& =\frac{8}{15}\left(v^{i} v^{j}-\frac{1}{3} \delta^{i j} v^{2}\right),
\end{aligned}
$$

where we have used (168) and $\mathcal{O}_{\ell>2}$ indicates all the integrals coming from the multipoles $\ell>2$ in the expansion (for $\ell=0$ and $\ell=2$ they vanish.) In fact we have dropped the $\mathcal{O}(\ell>2)$ since they are proportional to first-order photon moments $\ell>2$ which turn out to be supressed in the tight coupling limit. Finally, the term proportional to $(\mathbf{v} \cdot \mathbf{n}) \Delta_{2}^{(1)}\left(1-P_{2}(\hat{\mathbf{v}}\right.$. n)/5 gives a vanishing contribution. 
Collecting all the various pieces we find that the third moment of the R.H.S. of (134) is given by

$$
\begin{gathered}
-\frac{\tau^{\prime}}{2}\left[-\Pi_{\gamma}^{(2) i j}+\frac{1}{10} \Pi_{\gamma}^{(2) i j}+2 \delta_{e}^{(1)}\left(-\Pi_{\gamma}^{(1) i j}+\frac{1}{10} \Delta_{2}^{(1)}\right.\right. \\
\left.\times\left(\hat{v}^{i} \hat{v}^{j}-\frac{1}{3} \delta^{i j}\right)+\frac{12}{5}\left(v^{i} v^{j}-\frac{1}{3} \delta^{i j} v^{2}\right)\right] .
\end{gathered}
$$

Therefore, in the limit of tight coupling, when the interaction rate is very high, the second-order quadrupole moment turns out to be

$$
\Pi_{\gamma}^{(2) i j} \simeq \frac{8}{3}\left(v^{i} v^{j}-\frac{1}{3} \delta^{i j} v^{2}\right)
$$

by setting (221) to be vanishing (the term multiplying $\delta_{e}^{(1)}$ goes to zero in the tight coupling limit since it just comes from the first-order collision term). At linear order, one would simply get the term $9 \tau^{\prime} \Pi_{\gamma}^{(1) i j} / 10$ implying that, in the limit of a high scattering rate $\tau^{\prime}, \Pi_{\gamma}^{(1) i j}$ goes to zero. However at second-order the quadrupole is not suppressed in the tight coupling limit because it turns out to be sourced by the linear velocity squared. Indeed (222) has a very clear physical interpretation. If one describes the photons as a perfect fluid, at second-order in the perturbations the quadrupole one would get (by taking the traceless part of the energy momentum tensor) would be exactly given by (222) (see, e.g., [33]). Of course, this does not come as a surprise here, because the tight coupling limit exactly corresponds to a regime where the photons behave as a perfect fluid.

\subsection{Second-Order CMB Anisotropies Generated at Recom-} bination. The previous equations allow us to follow the evolution of the monopole and dipole of CMB photons at recombination. As at linear order, they will appear in the expression for the $\mathrm{CMB}$ anisotropies today $\Delta^{(2)}\left(k, n, \eta_{0}\right)$ together with various integrated effects. Our focus now will be to obtain an expression for the second-order $\mathrm{CMB}$ anisotropies today $\Delta^{(2)}\left(k, n, \eta_{0}\right)$ from which we can extract all those contributions generated specifically at recombination due to the nonlinear dynamics of the photon-baryon fluid. This expression will not only relate the moments $\Delta_{\ell m}^{(2)}$ today to the second-order monopole and dipole at recombination as it happens at linear order, but one has to properly account also for additional first-order squared contributions. Let us see how to achieve this goal in some details.

As we have seen previously, it is possible to write down an integral solution of the photon Boltzmann equation (134) in Fourier space. We wrote

$$
\begin{aligned}
& \Delta^{(2)^{\prime}}+i k \mu \Delta^{(2)}-\tau^{\prime} \Delta^{(2)}=e^{-i k \mu+\tau} \frac{d}{d \eta}\left[\Delta^{(2)} e^{i k \mu \eta-\tau}\right] \\
& =S(\mathbf{k}, \mathbf{n}, \eta)
\end{aligned}
$$

in order to derive a solution of the form

$$
\Delta^{(2)}\left(\mathbf{k}, \mathbf{n}, \eta_{0}\right)=\int_{0}^{\eta_{0}} d \eta S(\mathbf{k}, \mathbf{n}, \eta) e^{i k \mu\left(\eta-\eta_{0}\right)} e^{-\tau} .
$$

Here, $\mu=\cos \vartheta=\hat{\mathbf{k}} \cdot \mathbf{n}$ is the polar angle of the photon momentum in a coordinate system such that $\mathbf{e}_{3}=\hat{\mathbf{k}}$. At second-order the source term has been computed in [46] and can be read off (134) and (141) to be

$$
\begin{aligned}
S= & -\tau^{\prime} \Delta_{00}^{(2)}-4 n^{i} \Phi_{, i}^{(2)}+4 \Psi^{(2)^{\prime}}-8 \omega_{i}^{\prime} n^{i}-4 \chi_{i j}^{\prime} n^{i} n^{j} \\
& +8 \Delta^{(1)}\left(\Psi^{(1)^{\prime}}-n^{i} \Phi_{, i}^{(1)}\right)-2 n^{i}\left(\Phi^{(1)}+\Psi^{(1)}\right)\left(\Delta^{(1)}+4 \Phi^{(1)}\right)_{, i} \\
- & 2\left[\left(\Phi^{(1)}+\Psi^{(1)}\right)_{, j} n^{i} n^{j}-\left(\Phi^{(1)}+\Psi^{(1)}\right)^{, i}\right] \frac{\partial \Delta^{(1)}}{\partial n^{i}} \\
- & \tau^{\prime}\left[-\frac{1}{2} \sum_{m=-2}^{2} \frac{\sqrt{4 \pi}}{5^{3 / 2}} \Delta_{2 m}^{(2)} Y_{2 m}(\mathbf{n})+4 \mathbf{v}^{(2)} \cdot \mathbf{n}\right. \\
& +2 \delta_{e}^{(1)}\left(\Delta_{0}^{(1)}-\Delta^{(1)}+4 \mathbf{v} \cdot \mathbf{n}+\frac{1}{2} \Delta_{2}^{(1)} P_{2}(\hat{\mathbf{v}} \cdot \mathbf{n})\right) \\
& +2(\mathbf{v} \cdot \mathbf{n})\left[\Delta^{(1)}+3 \Delta_{0}^{(1)}-\Delta_{2}^{(1)}\left(1-\frac{5}{2} P_{2}(\widehat{\mathbf{v}} \cdot \mathbf{n})\right)\right] \\
& \left.-v \Delta_{1}^{(1)}\left(4+2 P_{2}(\widehat{\mathbf{v}} \cdot \mathbf{n})\right)+14(\mathbf{v} \cdot \mathbf{n})^{2}-2 v^{2}\right] .
\end{aligned}
$$

The key point here is to isolate all those terms that multiply the differential optical depth $\tau^{\prime}$. The reason is that in this case in the integral (224) one recognizes the visibility function $g(\eta)=-e^{-\tau} \tau^{\prime}$ which is sharply peaked at the time of recombination and whose integral over time is normalized to unity. Thus, for these terms the integral just reduces to the remaining integrand (apart from the visibility function) evaluated at recombination. The standard example that one encounters also at linear order is given by the first term appearing in the source $S,(225)$, that is $-\tau^{\prime} \Delta_{00}^{(2)}$. The contribution of this term to the integral (224) just reduces to

$$
\begin{aligned}
& \Delta^{(2)}\left(\mathbf{k}, \mathbf{n}, \eta_{0}\right) \\
& =\int_{0}^{\eta_{0}} d \eta e^{i k \mu\left(\eta-\eta_{0}\right)} e^{-\tau}\left(-\tau^{\prime}\right) \Delta_{00}^{(2)} \simeq e^{i k \mu\left(\eta_{*}-\eta_{0}\right)} \Delta_{00}^{(2)}\left(\eta_{*}\right),
\end{aligned}
$$

where $\eta_{*}$ is the epoch of recombination and, in the multipole decomposition (136), (226) brings the standard result

$$
\Delta_{\ell m}^{(2)}\left(\eta_{0}\right) \propto \Delta_{00}^{(2)}\left(\eta_{*}\right) j_{\ell}\left(k\left(\eta_{*}-\eta_{0}\right)\right),
$$

having used the Legendre expansion $e^{i \mathbf{k} \cdot \mathbf{x}}=\sum_{\ell}(i)^{\ell}(2 \ell+$ 1) $j_{\ell}(k x) P_{\ell}(\hat{\mathbf{k}} \cdot \widehat{\mathbf{x}})$. In (227), the monopole at recombination is found by solving the Boltzmann equations (207)-(212) derived in tight coupling limit. 
Looking at (225) we recognize immediately some terms which multiply explicitly $\tau^{\prime}$ (the first one discussed in the example above and the last two lines of (225)). However, it is easy to realize from the standard procedure adopted at the linear-order that such terms are not the only ones. This is clear by focusing, as an example, on the term $-4 n^{i} \Phi_{, i}^{(2)}$ in the source $S$ which appears in the same form also at linear order. In Fourier space one can replace the angle $\mu$ with a time derivative and thus this term gives rise to

$$
\begin{aligned}
& -4 i k \int_{0}^{\eta_{0}} d \eta e^{i k \mu\left(\eta-\eta_{0}\right)} e^{-\tau} \mu \Phi^{(2)} \\
& \quad=-4 \int_{0}^{\eta_{0}} d \eta \Phi^{(2)} e^{-\tau} \frac{d}{d \eta}\left(e^{i k \mu\left(\eta-\eta_{0}\right)}\right) \\
& =4 \int_{0}^{\eta_{0}} d \eta e^{i k \mu\left(\eta-\eta_{0}\right)} e^{-\tau}\left(\Phi^{(2)^{\prime}}-\tau^{\prime} \Phi^{(2)}\right),
\end{aligned}
$$

where, in the last step, we have integrated by parts. In (228) the time derivative of the gravitational potential contributes to the Integrated Sachs-Wolfe effect, but also also a $\tau^{\prime}$ results implying that we have also to evaluate $\Phi^{(2)}$ at recombination. Thus, in the following, we look for those terms in the source (225) which give rise to a $\tau^{\prime}$ factor in the same way as for $-4 n^{i} \Phi_{, i}^{(2)}$. In particular, let us consider the combination in (225)

$$
\begin{aligned}
C & \equiv 8 \Delta^{(1)}\left(\Psi^{(1)^{\prime}}-n^{i} \Phi_{, i}^{(1)}\right)-2 n^{i}\left(\Phi^{(1)}+\Psi^{(1)}\right)\left(\Delta^{(1)}+4 \Phi^{(1)}\right)_{, i} \\
& =8 \Delta^{(1)} \Psi^{(1)^{\prime}}-8 n^{i}\left(\Delta^{(1)} \Phi^{(1)}\right)_{, i}+4 \Phi^{(1)} n^{i} \Delta_{, i}^{(1)}-8 n^{i}\left(\Phi^{(1) 2}\right)_{, i}
\end{aligned}
$$

where for simplicity we are setting $\Phi^{(1)} \simeq \Psi^{(1)}$. We already recognize terms of the form $n^{i} \partial_{i}(\cdot)$. Moreover we can use the Boltzmann equation (129) to replace $n^{i} \Delta_{, i}^{(1)}$ in (95). This brings

$$
\begin{aligned}
C= & 8 \Delta^{(1)} \Psi^{(1)^{\prime}}-4 \Psi^{(1)} \Delta^{(1)^{\prime}}-8\left(\Psi^{(1) 2}\right)^{\prime} \\
& -8 n^{i}\left(\Delta^{(1)} \Phi^{(1)}+2 \Phi^{(1) 2}\right)_{, i} \\
& -4 \tau^{\prime} \Psi^{(1)}\left[\Delta_{00}^{(1)}-\Delta^{(1)}+4 \mathbf{v}^{(1)} \cdot \mathbf{n}+\frac{1}{2} \Delta_{2}^{(1)} P_{2}(\hat{\mathbf{v}} \cdot \mathbf{n})\right]
\end{aligned}
$$

In fact, we will not be interested for our purposes in the first three terms of (230), since they will not contribute to the anisotropies generated at recombination.
Therefore, as a result of (225), (228) and (230), we can rewrite the source term (225) as

$$
S=S_{*}+S^{\prime}
$$

where

$$
\begin{aligned}
S_{*}=-\tau^{\prime}[ & \Delta_{00}^{(2)}+4 \Phi^{(2)}-\frac{1}{2} \sum_{m=-2}^{2} \frac{\sqrt{4 \pi}}{5^{3 / 2}} \Delta_{2 m}^{(2)} Y_{2 m}(\mathbf{n}) \\
& +4 \mathbf{v}^{(2)} \cdot \mathbf{n}+2 \delta_{e}^{(1)} \\
& \times\left(\Delta_{0}^{(1)}-\Delta^{(1)}+4 \mathbf{v} \cdot \mathbf{n}+\frac{1}{2} \Delta_{2}^{(1)} P_{2}(\hat{\mathbf{v}} \cdot \mathbf{n})\right) \\
& +2(\mathbf{v} \cdot \mathbf{n})\left[\Delta^{(1)}+3 \Delta_{0}^{(1)}-\Delta_{2}^{(1)}\left(1-\frac{5}{2} P_{2}(\hat{\mathbf{v}} \cdot \mathbf{n})\right)\right] \\
& -v \Delta_{1}^{(1)}\left(4+2 P_{2}(\hat{\mathbf{v}} \cdot \mathbf{n})\right)+14(\mathbf{v} \cdot \mathbf{n})^{2} \\
& -2 v^{2}+8 \Delta^{(1)} \Phi^{(1)}+16 \Phi^{(1) 2}+4 \Psi^{(1)} \\
& \times\left[\Delta_{0}^{(1)}-\Delta^{(1)}+4 \mathbf{v}^{(1)} \cdot \mathbf{n}+\frac{1}{2} \Delta_{2}^{(1)} P_{2}(\hat{\mathbf{v}} \cdot \mathbf{n})\right] \\
S^{\prime}=4( & \left.\Phi^{(2)}+\Psi^{(2)}\right)^{\prime}-8 \omega^{\prime}{ }_{i} n^{i}-4 \chi_{i j}^{\prime} n^{i} n^{j} \\
-2\left[\left(\Phi^{(1)}+\Psi^{(1)}\right), n^{i} n^{j}-\left(\Phi^{(1)}+\Psi^{(1)}\right)^{, i}\right] \frac{\partial \Delta^{(1)}}{\partial n^{i}} & 8\left(\Delta^{(1)} \Phi^{(1)}\right)^{\prime}+8 \Delta^{(1)} \Psi^{(1)^{\prime}}-4 \Psi^{(1)} \Delta^{(1)}+16 \Psi^{(1)} \Psi^{(1)^{\prime}}
\end{aligned}
$$

In (231), $S_{*}$ contains the contribution to the second-order $\mathrm{CMB}$ anisotropies created on the last scattering surface at recombination, while $S^{\prime}$ includes all those effects which are integrated in time from the last scattering surface up to now, including the second-order integrated Sachs-Wolfe effect and the second-order lensing effect. Since the main concern of this Section is the CMB anisotropies generated at last scattering, from now on we will focus only on the contribution from the last scattering surface $S_{*}$. In particular, notice that following the same steps that lead to (226), the first two terms of (232) give rise to the CMB temperature anisotropies as written in (202).

7.4. Tightly Coupled Solutions for the Second-Order Perturbations. In this section, we will solve the tightly coupled limit of the Boltzmann equations (207) and (209) at second-order in perturbation theory. We will proceed as for the linear case, focusing on the two limiting cases of perturbation modes entering the horizon, respectively, much before and much after the time of equality. The solution of (214) can be 
written as

$$
\begin{aligned}
{[1} & +R(\eta)]^{1 / 4}\left(\Delta_{00}^{(2)}-4 \Psi^{(2)}\right) \\
= & A \cos \left[k r_{s}(\eta)\right]+B \sin \left[k r_{s}(\eta)\right] \\
& -4 \frac{k}{\sqrt{3}} \int_{0}^{\eta} d \eta^{\prime}\left[1+R\left(\eta^{\prime}\right)\right]^{3 / 4} \\
& \times\left(\Phi^{(2)}\left(\eta^{\prime}\right)+\frac{\Psi^{(2)}\left(\eta^{\prime}\right)}{1+R}\right) \sin _{k}\left[\eta, \eta^{\prime}\right] \\
& +\frac{\sqrt{3}}{k} \int_{0}^{\eta} d \eta^{\prime}\left[1+R\left(\eta^{\prime}\right)\right]^{3 / 4} \\
& \times\left(\S^{\prime} \Delta+\frac{\mathscr{H} R}{1+R} \S_{\Delta}-\frac{4}{3} i k_{i} \S_{V}^{i}\right) \sin _{k}\left[\eta, \eta^{\prime}\right],
\end{aligned}
$$

where for simplicity, we use the shorthand notation $\sin _{k}\left[\eta, \eta^{\prime}\right] \equiv \sin \left[k\left(r_{s}(\eta)-r_{s}\left(\eta^{\prime}\right)\right)\right]$, and $r_{s}(\eta)$ is the sound horizon

$$
r_{s}(\eta)=\int_{0}^{\eta} d \eta^{\prime} c_{s}\left(\eta^{\prime}\right)
$$

The source terms are given in (208) and (213). Notice that we can write ${\delta^{\prime}}_{\Delta}+(\mathscr{H} R /(1+R)) \delta_{\Delta}=\left(\delta_{\Delta}(1+R)\right)^{\prime} / 1+R$ so that we can perform an integration by parts in (234) leading to

$$
\begin{aligned}
{[1} & +R(\eta)]^{1 / 4}\left(\Delta_{00}^{(2)}-4 \Psi^{(2)}\right) \\
= & A \cos \left[k r_{s}(\eta)\right]+B \sin \left[k r_{s}(\eta)\right] \\
& -4 \frac{k}{\sqrt{3}} \int_{0}^{\eta} d \eta^{\prime}\left[1+R\left(\eta^{\prime}\right)\right]^{3 / 4}\left(\Phi^{(2)}\left(\eta^{\prime}\right)+\frac{\Psi^{(2)}\left(\eta^{\prime}\right)}{1+R}\right) \\
& \times \sin _{k}\left[\eta, \eta^{\prime}\right]+\int_{0}^{\eta} d \eta^{\prime} s_{\Delta}\left(\eta^{\prime}\right)\left(1+R\left(\eta^{\prime}\right)\right) \frac{1}{4} \cos _{k}\left[\eta, \eta^{\prime}\right] \\
& -\frac{\sqrt{3}}{k} s_{\Delta}(0) \sin \left[k r_{s}(\eta)\right]-\frac{4}{\sqrt{3}} \frac{i k_{i}}{k} \int_{0}^{\eta} d \eta^{\prime} 8_{V}^{i}\left(\eta^{\prime}\right) \\
& \times\left(1+R\left(\eta^{\prime}\right)\right)^{3 / 4} \sin _{k}\left[\eta, \eta^{\prime}\right]+\frac{\sqrt{3}}{4 k} \int_{0}^{\eta} d \eta^{\prime} 8_{\Delta}\left(\eta^{\prime}\right) \\
& \times\left(1+R\left(\eta^{\prime}\right)\right)^{-1 / 4} R^{\prime}\left(\eta^{\prime}\right) \sin _{k}\left[\eta, \eta^{\prime}\right] .
\end{aligned}
$$

In order to give an analytical solution that catches most of the physics underlying (234) and which remains at the same time very simple to treat, we will make some simplifications which are also usually adopted in linear theory, see for example, [73]. In particular, we will treat $R=R_{*}$ as a constant evaluated at the time of recombination. In this way, with the presence of $R$ in the varying cosines and sines, we can keep track of a damping of the photon velocity amplitude with respect to the case $R=0$ which prevents the acoustic peaks in the power-spectrum to disappear. Treating $R$ as a constant is justified by the fact that for modes within the horizon the time scale of the oscillations is much shorter than the time scale on which $R$ varies. If $R$ is a constant the sound speed is just a constant

$$
c_{\mathrm{s}}=\frac{1}{\sqrt{3\left(1+R_{*}\right)}},
$$

and the sound horizon is simply $r_{s}(\eta)=c_{s} \eta$.

There is also another good reason not to forget about $R$, even though $R<1$, which is particularly relevant when estimating the second-order contributions to the CMB anisotropies in (202). As we will see in Section 9, that combination on small scales comes actually as proportional to $R$, so that neglecting $R$ would lead to miss one of the most significant $\mathrm{CMB}$ contribution at second order.

Another simplification consists in solving the evolutions of the perturbations in two well distinguished limiting regimes. One regime is for those perturbations which enter the Hubble radius when matter is the dominant component, that is at times much bigger than the equality epoch, with $k \ll k_{\mathrm{eq}} \sim \eta_{\mathrm{eq}}^{-1}$, where $k_{\mathrm{eq}}$ is the wavenumber of the Hubble radius at the equality epoch. The other regime is for those perturbations with much smaller wavelengths which enter the Hubble radius when the universe is still radiation dominated, that is perturbations with wavenumbers $k \gg$ $k_{\mathrm{eq}} \sim \eta_{\mathrm{eq}}^{-1}$. In fact, we are interested in perturbation modes which are within the horizon by the time of recombination $\eta_{*}$. Therefore, we will further suppose that $\eta_{*} \gg \eta_{\mathrm{eq}}$ in order to study such modes in the first regime. Even though $\eta_{*} \gg \eta_{\mathrm{eq}}$ is not the real case, it allows to obtain some analytical expressiions.

7.4.1. Setting the Initial Conditions: Primordial NonGaussianity. The integration constants $A$ and $B$ are fixed according to the initial conditions for the second-order cosmological perturbations. These refer to the values of the perturbations on superhorizon scales deep in the radiation dominated period. We will consider the case of initial adiabatic perturbations, for which there exist some useful conserved quantities on large scales which as such carry directly the information about the initial conditons.

As explained in Section 4, such a conserved quantity is given by the curvature perturbation $\zeta$ and its conserved value allows to set the initial conditions for the metric and matter perturbations accounting for the primordial contributions. At linear order during the radiation-dominated epoch and on large scales $\zeta^{(1)}=-2 \Psi^{(1)} / 3$. On the other hand, after some calculations, one can easily compute $\Delta \zeta^{(2)}$ for a radiation dominated epoch

$$
\Delta \zeta^{(2)}=\frac{7}{2}\left(\Psi^{(1)}\right)^{2}
$$

where in (37) one uses that on large scales $\delta^{(1)} \rho_{\gamma} / \rho_{\gamma}=-2 \Psi^{(1)}$ and the energy continuity equation $\delta^{(1)^{\prime}} \rho_{\gamma}+4 \mathcal{H} \delta^{(1)} \rho_{\gamma}-$ $4 \Psi^{(1)^{\prime}} \rho_{\gamma}=0$. Therefore, we find

$$
\zeta^{(2)}=-\Psi^{(2)}+\frac{\Delta_{00}^{(2)}}{4}+\frac{7}{2} \Psi^{(1) 2}(0),
$$


where we are evaluating the quantities in the large scale limit for $\eta \rightarrow 0$. Using the parametrization (38) at the initial times the quantity $\Delta_{00}^{(2)}-4 \Psi^{(2)}$ is given by

$$
\Delta_{00}^{(2)}-4 \Psi^{(2)}=2\left(9 a_{\mathrm{NL}}-7\right) \Psi^{(1) 2}(0) .
$$

Since for adiabatic perturbations such a quantity is conserved on superhorizon scales, it follows that the constant $B=0$ and $A=2\left(9 a_{\mathrm{NL}}-7\right) \Psi^{(1) 2}(0)$.

Equations (234) and (236) are analytical expressions describing the acoustic oscillations of the photon-baryon fluid induced at second-order for perturbation modes within the horizon at recombination. In the following, we will adopt similar simplifications already used for the linear case in order to provide some analytical solutions. In particular, if in (236) we treat $R$ as a constant we can write, using the initial conditions determined above,

$$
\begin{aligned}
\left(\Delta_{00}^{(2)}-4 \Psi^{(2)}\right) & \\
= & 2\left(9 a_{\mathrm{NL}}-7\right) \Psi^{(1) 2}(0) \cos \left[k r_{s}(\eta)\right] \\
& -4 k c_{s} \int_{0}^{\eta} d \eta^{\prime}\left(\Phi^{(2)}\left(\eta^{\prime}\right)+\Psi^{(2)}\left(\eta^{\prime}\right)+R \Phi^{(2)}\right) \sin _{k}\left[\eta, \eta^{\prime}\right] \\
& +\int_{0}^{\eta} d \eta^{\prime} s_{\Delta}\left(\eta^{\prime}\right) \cos _{k}\left[\eta, \eta^{\prime}\right]-\frac{\sqrt{3}}{k} s_{\Delta}(0) \sin \left[k r_{s}(\eta)\right] \\
& -\frac{4}{3} \frac{i k_{i}}{k c_{s}} \int_{0}^{\eta} d \eta^{\prime} \delta_{V}^{i}\left(\eta^{\prime}\right) \sin _{k}\left[\eta, \eta^{\prime}\right] .
\end{aligned}
$$

7.5. Perturbation Modes with $k \gg k_{\text {eq }}$. In order to study the contribution to the second-order CMB anisotropies coming from perturbation modes that enter the horizon during the radiation dominated epoch, we will assume that the second-order gravitational potentials are the ones of a pure radiation dominated universe throughout the evolution. Though not strictly correct, this approximation will give us the basic picture of the acoustic oscillations for the baryon-photon fluid occurring for these modes. Also for the second-order case, in Section 7.7 we will provide the appropriate corrections accounting for the transition from radiation to matter domination which is indeed (almost) achieved by the recombination epoch. Before moving into the details a note of caution is in order here. At second order in the perturbations all the relevant quantities are expressed as convolutions of linear perturbations, bringing to a mode-mode mixing. In some cases, in our treatment for a given regime under analysis $\left(k \gg k_{\text {eq }}\right.$ or $k \ll$ $k_{\text {eq }}$ ) we use for the first-order perturbations the solutions corresponding to that particular regime, while the modemode mixing would require to consider in the convolutions (where one is integrating over all the wavenumbers) a more general expression for the first-order perturbations (which analytically does not exist anyway). For the computation of the $\mathrm{CMB}$ bispectrum this would be equivalent to consider just some specific scales, that is, all the three scales involved in the bispectrum should correspond approximately to wavenumbers $k \gg k_{\mathrm{eq}}$ or $k \ll k_{\mathrm{eq}}$, and not a combination of the two regimes (a step towards the evaluation of the threepoint correlation function has been taken on [45] where it was computed in the in so-called squeezed triangle limit, when one mode has a wavelength much larger than the other two and is outside the horizon).

In Appendix C, we show how to solve the Boltzmann equations at the linear level in the various regimes. In fact, for $k \gg k_{\text {eq }}$ the acoustic oscillations can be solved in an alternative way (see [47]) for this procedure at linear order). One can start directly from (207), where we can neglect the gravitational potential term $\Psi^{(2)^{\prime}}$. The reason is that, as it happens at linear order, the second-order gravitational potentials decay at late times as $\eta^{-2}$, while the second-order velocity $v_{\gamma}^{(2) i}$ oscillates in time. Let us now see that in some details.

The evolution equation for the gravitational potential $\Psi^{(2)}$ is given by (B.13) and is characterized by the source term $S_{\gamma}$, (B.15). In particular, the source term contains the secondorder quadrupole moment of the photons $\Pi_{\gamma}^{(2) i j}$. We saw in Section 7.2.3 that at second-order the quadrupole moment is not suppressed in the tight coupling limit, being fed by the nonlinear combination of the first-order velocities, (222). For the perturbation modes, we are considering here the velocity at late times is oscillating being given in Appendix C in Fourier space. Since the linear gravitational potential decays in time and for a radiation dominated period $\mathscr{H}=$ $1 / \eta$, it is easy to check that the dominant contribution at late times to the source term $S_{\gamma}$ simply reduces to

$$
\begin{aligned}
S_{\gamma} & \simeq \frac{3}{2} \mathcal{H}^{2} \frac{\partial_{i} \partial^{j}}{\nabla^{2}} \Pi_{\gamma j}^{(2) i} \\
& \equiv \frac{F\left(\mathbf{k}_{1}, \mathbf{k}_{2}, \mathbf{k}\right)}{\eta^{2}} C \Psi_{\mathbf{k}_{1}}^{(1)}(0) \Psi_{\mathbf{k}_{2}}^{(1)}(0) \sin \left(k_{1} c_{s} \eta\right) \sin \left(k_{2} c_{s} \eta\right),
\end{aligned}
$$

where

$$
C=-\frac{9}{c_{s}^{2} k_{1} k_{2}}
$$

and the sound speed is $c_{s}=1 / \sqrt{3(1+R)}$. Before proceeding further let us explain the notation that we are using. The equivalence symbol will be used to indicate that we are evaluating the expression in Fourier space. At second-order in perturbation theory most of the Fourier transforms reduce to some convolutions. We will not indicate these convolutions explicitly but just through their kernel. For example in (242) by $F\left(\mathbf{k}_{1}, \mathbf{k}_{2}, \mathbf{k}\right)$, we actually indicate the convolution operator

$$
F \equiv \frac{1}{2 \pi^{3}} \int d^{3} k_{1} d^{3} k_{2} \delta^{(1)}\left(\mathbf{k}_{1}+\mathbf{k}_{2}-\mathbf{k}\right) F\left(\mathbf{k}_{1}, \mathbf{k}_{2}, \mathbf{k}\right) .
$$

In the specific case of (242) the kernel is given by

$$
F\left(\mathbf{k}_{1}, \mathbf{k}_{2}, \mathbf{k}\right)=\frac{\left(\mathbf{k} \cdot \mathbf{k}_{1}\right)\left(\mathbf{k} \cdot \mathbf{k}_{2}\right)}{k^{2}}-\frac{1}{3} \mathbf{k}_{1} \cdot \mathbf{k}_{2} .
$$

The choice of these conventions is due not only for simplicity and to keep our expressions shorter, but also because at the 
end we will be interested to the bispectrum of the CMB anisotropies generated at recombination, and the relevant expressions entering in the bispectrum are just the kernels of the convolution integrals.

Having determined the leading contribution to the source term at late times, we can now solve the evolution equation (B.13). Since the source, term scales like $\eta^{-2}$, it is useful to introduce the rescaled variable $\chi=\eta^{2} \Psi^{(2)}$. Equation (B.13) then reads

$$
\chi^{\prime \prime}+\left(k^{2} c_{s}^{2}-\frac{2}{\eta^{2}}\right) \chi=\eta^{2} S_{\gamma}
$$

For perturbation modes which are subhorizon with $k \eta \gg 1$ the solution of the homogeneous equation is given by

$$
\chi_{\text {hom. }}=A \cos \left(k c_{s} \eta\right)+B \sin \left(c_{s} k \eta\right)
$$

from which we can build the general solution

$$
\begin{aligned}
\chi= & \chi_{\text {hom. }}+\chi_{+} \int_{0}^{\eta} d \eta^{\prime} \frac{\chi_{-}\left(\eta^{\prime}\right)}{W\left(\eta^{\prime}\right)} S_{\gamma}\left(\eta^{\prime}\right) \\
& -\chi_{-} \int_{0}^{\eta} d \eta^{\prime} \frac{\chi_{+}\left(\eta^{\prime}\right)}{W\left(\eta^{\prime}\right)} S_{\gamma}\left(\eta^{\prime}\right)
\end{aligned}
$$

where $W=-k c_{s}$ is the Wronskian, and $\chi_{+}=\cos \left(k c_{s} \eta\right), \chi_{-}=$ $\sin \left(c_{s} k \eta\right)$. Using (242), the integrals involve products of sines and cosines which can be performed giving

$$
\begin{aligned}
\chi= & \chi_{\text {hom. }}-\frac{F C}{c_{s}^{2}} \Psi_{\mathbf{k}_{1}}^{(1)}(0) \Psi_{\mathbf{k}_{2}}^{(1)}(0) \\
& \times\left(k \left[2 k_{1} k_{2} \cos \left(k_{1} c_{s} \eta\right) \cos \left(k_{2} c_{s} \eta\right)-2 k_{1} k_{2}\right.\right. \\
& \left.\left.\quad \times \cos \left(k c_{s} \eta\right)+\left(k_{1}^{2}+k_{2}^{2}-k^{2}\right) \sin \left(k_{1} c_{s} \eta\right) \sin \left(k_{2} c_{s} \eta\right)\right]\right) \\
& /\left(k_{1}^{4}+k_{2}^{4}+k^{4}-2 k_{1}^{2} k_{2}^{2}-2 k_{1}^{2} k^{2}-2 k_{2}^{2} k^{2}\right)
\end{aligned}
$$

Thus, the gravitational potential $\Psi^{(2)}$ at late times is given by

$$
\begin{aligned}
& \Psi_{\mathbf{k}}^{(2)}(\eta) \\
& =-3 \Psi^{(2)}(0) \frac{\cos \left(k c_{s} \eta\right)}{\left(k c_{s} \eta\right)^{2}}-\frac{F C}{\eta^{2} c_{s}^{2}} \Psi_{\mathbf{k}_{1}}^{(1)}(0) \Psi_{\mathbf{k}_{2}}^{(1)}(0) \\
& \quad \times\left(\left[2 k_{1} k_{2} \cos \left(k_{1} c_{s} \eta\right) \cos \left(k_{2} c_{s} \eta\right)-2 k_{1} k_{2} \cos \left(k c_{s} \eta\right)\right.\right. \\
& \left.\left.\quad+\left(k_{1}^{2}+k_{2}^{2}-k^{2}\right) \sin \left(k_{1} c_{s} \eta\right) \sin \left(k_{2} c_{s} \eta\right)\right]\right) \\
& /\left(k_{1}^{4}+k_{2}^{4}+k^{4}-2 k_{1}^{2} k_{2}^{2}-2 k_{1}^{2} k^{2}-2 k_{2}^{2} k^{2}\right),
\end{aligned}
$$

where we have set the integration constant $B=0$ and $A=-3 \Psi^{(2)}(0) /\left(k c_{s}\right)^{2}$ in order to match the homogeneous solution at late times. Here, $\Psi^{(2)}(0)$ is the intial condition for the gravitational potential taken on large scales deep in the radiation dominated era which will be determined in Section 7.5.2.
Equation (250) shows the result that we anticipated: also at second order the gravitational potential varies in time oscillating with an amplitude that decays as $\eta^{-2}$. Let us then take the divergence of the $(i-0)$ Einstein equation (30) expanded at second order

$$
\begin{aligned}
& \partial_{i}\left[\frac{1}{2} \partial^{i} \Psi^{(2)^{\prime}}+\frac{\mathscr{H}}{2} \partial^{i} \Phi^{(2)}+2 \Psi^{(1)} \partial^{i} \Psi^{(1)^{\prime}}\right. \\
& \left.\quad+2 \mathscr{H} \Psi^{(1)} \partial^{i} \Phi^{(1)}-\Psi^{(1)^{\prime}} \partial^{i} \Phi^{(1)}\right] \\
& =-2 \mathscr{H}^{2} \partial_{i}\left[\frac{1}{2} v_{\gamma}^{(2) i}+\left(\Phi^{(1)}+\Psi^{(1)}\right) v_{\gamma}^{(1) i}+\Delta_{00}^{(1)} v_{\gamma}^{(1) i}\right],
\end{aligned}
$$

which, using the first-order $(i-0)$ Einstein equation and $\Phi^{(1)} \simeq \Psi^{(1)}$, reduces to

$$
\begin{gathered}
\partial_{i}\left[\frac{1}{2} \partial^{i} \Psi^{(2)^{\prime}}+\frac{\mathscr{H}}{2} \partial^{i} \Phi^{(2)}-\Psi^{(1)^{\prime}} \partial^{i} \Psi^{(1)}\right] \\
=-2 \mathcal{H}^{2} \partial_{i}\left[\frac{1}{2} v_{\gamma}^{(2) i}+\Delta_{00}^{(1)} v_{\gamma}^{(1) i}\right] .
\end{gathered}
$$

Since $\Psi^{(1)}$ during a radiation dominated period is given by (B.11) and at late times it decays oscillating, it is easy to see that $\left(\Psi^{(1)^{\prime}} \partial^{i} \Psi^{(1)}\right)$ will be oscillating and decaying as $\eta^{-4}$ and thus can be neglected with respect to $\Psi^{(2)^{\prime}}$, which oscillates with an amplitude decaying as $\eta^{-2}$. Also, $\mathscr{H} \Phi^{(2)}$ turns out to be subdominant. Recall that $\Phi^{(2)}=\Psi^{(2)}-Q^{(2)}$ and $Q^{(2)}$ is dominated by the second-order quadrupole of the photons in (B.15), so that $\Phi^{(2)}$ scales like $\Psi^{(2)}$ but there is the additional damping factor of the Hubble rate $\mathscr{H}=1 / \eta$. Thus, the dominant terms give

$$
\partial_{i} v_{\gamma}^{(2) i} \simeq-\frac{1}{2 \mathscr{H}^{2}} \nabla^{2} \Psi^{(2)^{\prime}}-2 \partial_{i}\left(\Delta_{00}^{(1)} v_{\gamma}^{(1) i}\right)
$$

Equation (253) allows to proceed further in a similar way as for the linear case by using the results found so far, (250) and (253), in the energy continuity equation (207). In (207) the first- and second-order gravitational potentials can be neglected with respect to the remaining terms given by $\Delta_{00}^{(1)}$ and $v_{\gamma}^{(1) i}$ which oscillate in time. Thus, replacing the divergence of the second-order velocity by the expression (253), (207) becomes

$$
\Delta_{00}^{(2)^{\prime}}=\frac{2}{3 \mathscr{H}^{2}} \nabla^{2} \Psi^{(2)^{\prime}}+\frac{8}{3} \partial_{i} v_{\gamma}^{(1) i} \Delta_{00}^{(1)}+\left(\Delta_{00}^{(1) 2}\right)^{\prime}
$$

which, using the first-order equation (C.1), further simplifies to

$$
\Delta_{00}^{(2)^{\prime}}=\frac{2}{3 \mathscr{H}^{2}} \nabla^{2} \Psi^{(2)^{\prime}}
$$

where we have kept only the dominant terms at late times. 
The gravitational potential $\Psi^{(2)}$ is given in (250), so the integration of (255) gives

$$
\begin{aligned}
\Delta_{00}^{(2)}= & 6 \Psi^{(2)}(0) \cos \left(k c_{s} \eta\right)+2 \frac{F C}{3 c_{s}^{2}} \Psi_{\mathbf{k}_{1}}^{(1)}(0) \Psi_{\mathbf{k}_{2}}^{(1)}(0) k^{2} \\
\times & \left(\left[2 k_{1} k_{2} \cos \left(k_{1} c_{s} \eta\right) \cos \left(k_{2} c_{s} \eta\right)-2 k_{1} k_{2} \cos \left(k c_{s} \eta\right)\right.\right. \\
& \left.\left.+\left(k_{1}^{2}+k_{2}^{2}-k^{2}\right) \sin \left(k_{1} c_{s} \eta\right) \sin \left(k_{2} c_{s} \eta\right)\right]\right) \\
& /\left(k_{1}^{4}+k_{2}^{4}+k^{4}-2 k_{1}^{2} k_{2}^{2}-2 k_{1}^{2} k^{2}-2 k_{2}^{2} k^{2}\right) .
\end{aligned}
$$

Needless to say, modes for $k \gg k_{D}$, where $k_{D}^{-1}$ indicates the usual damping length, are supposed to be multiplied by an exponential $e^{-\left(k / k_{D}\right)^{2}}$ (see, e.g., [70]).

7.5.1. Vector Perturbations. So far, we have discussed only scalar perturbations. However, at second-order in perturbation theory an unavoidable prediction is that also vector (and tensor) perturbation modes are produced dynamically as nonlinear combination of first-order scalar perturbations. In particular notice that the second-order velocity appearing in (232), giving rise to a second-order Doppler effect at last scattering, will contain a scalar and a vector (divergence free) part. Equation (253) provides the scalar component of the second-order velocity. We now derive an expression for the velocity that includes also the vector contribution.

The (second-order) vector metric perturbation $\omega^{i}$ when radiation dominates can be obtained from (B.17)

$$
-\frac{1}{2} \nabla^{2} \omega^{i}+3 \mathscr{H}^{2} \omega^{i}=-4 \mathscr{H}^{2}\left(\delta_{j}^{i}-\frac{\partial^{i} \partial_{j}}{\nabla^{2}}\right)\left(\frac{v_{\gamma}^{(2) j}}{2}+\Delta_{00}^{(1)} v_{\gamma}^{(1) j}\right),
$$

where we have dropped the gravitational potentials $\Psi^{(1)} \simeq$ $\Phi^{(1)}$ which are subdominant at late times. On the other hand, from the velocity continuity equation (212), we get

$$
\begin{aligned}
& v_{\gamma}^{(2) i^{\prime}}+\frac{1}{4} \Delta_{00}^{(2), i} \\
& =\frac{1}{4}\left(\Delta_{00}^{(1) 2}\right)^{, i}+\frac{8}{3} v_{\gamma}^{(1) i} \partial_{j} v_{\gamma}^{(1) j}-2 \omega^{i^{\prime}}-\frac{3}{4} \partial_{k} \Pi_{\gamma}^{(2) k i},
\end{aligned}
$$

neglecting the term proportional to $R$ and the decaying gravitational potentials. Using the tight coupling equations at first order and integrating over time, one finds

$v_{\gamma}^{(2) i}+2\left(v_{\gamma}^{(1) i} \Delta_{00}^{(1)}\right)=-2 \omega^{i}-\frac{1}{4} \int d \eta^{\prime} \Delta_{00}^{(2), i}-\frac{3}{4} \int d \eta^{\prime} \partial_{k} \Pi_{\gamma}^{(2) k i}$.

We can thus plug (259) into (257) to find that at late times (for $k \eta \gg 1$ )

$$
\nabla^{2} \omega^{i}=-3 \mathscr{H}^{2}\left(\delta_{j}^{i}-\frac{\partial^{i} \partial_{j}}{\nabla^{2}}\right) \int d \eta^{\prime} \partial_{k} \Pi_{\gamma}^{(2) k j}
$$

We will come later to the explicit expression for the term on the R.H.S. of (260). Here, it is enough to notice that the second-order quadrupole oscillate in time and thus $\omega^{i}$ will decay in time as $\mathscr{H}^{2}=1 / \eta^{2}$. This shows that $\omega^{i}$ in (259) can be in fact neglected with respect to the other terms giving

$$
v_{\gamma}^{(2) i}=-2\left(v_{\gamma}^{(1) i} \Delta_{00}^{(1)}\right)-\frac{1}{4} \int d \eta^{\prime} \Delta_{00}^{(2), i}-\frac{3}{4} \int d \eta^{\prime} \partial_{k} \Pi_{\gamma}^{(2) k i} .
$$

It can be useful to compute the combination on the R.H.S. of (260) $\left(\delta_{j}^{i}-\partial^{i} \partial_{j} / \nabla^{2}\right) \partial_{k} \Pi_{\gamma}^{(2) k j}$. The second-order quadrupole moment of the photons in the tightly coupled limit is given by (222), and

$$
\partial_{k} \Pi_{\gamma}^{(2) k j}=\frac{8}{3}\left[\partial_{k}\left(v^{k} v^{j}\right)-2 v^{k} \partial^{j} v_{k}\right]=\frac{8}{3}\left[v^{j} \partial_{k} v^{k}-v^{k} \partial^{j} v_{k}\right],
$$

where in the last step, we have used that the linear velocity is the gradient of a scalar perturbation. We thus find

$$
\begin{aligned}
& \left(\delta_{j}^{i}-\frac{\partial^{i} \partial_{j}}{\nabla^{2}}\right) \partial_{k} \Pi_{\gamma}^{(2) k j} \\
& =\frac{8}{3}\left(v^{i} \partial_{k} v^{k}-v^{k} \partial^{i} v_{k}\right)-\frac{8}{3} \frac{\partial^{i}}{\nabla^{2}} \\
& \quad \times\left[\left(\partial_{k} v^{k}\right)^{2}+v^{j} \partial_{j} \partial_{k} v^{k}-\partial_{j} v^{k} \partial^{j} v_{k}-v^{k} \nabla^{2} v_{k}\right] .
\end{aligned}
$$

Notice that if we split the quadrupole moment into a scalar, vector (divergence-free) and tensor (divergence-free and traceless) parts as

$$
\Pi_{\gamma}^{(2) k j}=\Pi_{\gamma}^{(2), k j}-\frac{1}{3} \nabla^{2} \delta^{k j} \Pi_{\gamma}^{(2)}+\Pi_{\gamma}^{(2) k, j}+\Pi_{\gamma}^{(2) j, k}+\Pi_{\gamma T}^{(2) k j},
$$

then it turns out that

$$
\left(\delta_{j}^{i}-\frac{\partial^{i} \partial_{j}}{\nabla^{2}}\right) \partial_{k} \Pi_{\gamma}^{(2) k j}=\nabla^{2} \Pi_{\gamma}^{(2) i},
$$

where $\Pi_{\gamma}^{(2) i}$ is the vector part of the quadrupole moment. Therefore, one can rewrite (260) as

$$
\omega^{i}=-3 \mathscr{H}^{2} \int d \eta^{\prime} \Pi_{\gamma}^{(2) i}
$$

7.5.2. Initial Conditions for the Second-Order Gravitational Potentials. In order to complete the study of the CMB anisotropies at second-order for modes $k \gg k_{\text {eq }}$, we have to specify the initial conditions $\Psi^{(2)}(0)$ appearing in (256). These are set on superhorizon scales deep in the standard radiation dominated epoch (for $\eta \rightarrow 0$ ) by exploiting the conservation in time of the curvature perturbation $\zeta$. On superhorizon scales, $\zeta^{(2)}$ is given by (239) during the radiation dominated epoch and, using the $(0-0)$ Einstein equation in the large scale limit $\Delta_{00}^{(2)}=-2 \Phi^{(2)}+4 \Phi^{(1) 2}$, we find

$$
\zeta^{(2)}=-\frac{3}{2} \Psi^{(2)}(0)-\frac{1}{2}\left(\Phi^{(2)}(0)-\Psi^{(2)}(0)\right)+\frac{9}{2} \Psi^{(1) 2}(0) .
$$


The conserved value of $\zeta^{(2)}$ is parametrized by $\zeta^{(2)}=$ $2 a_{\mathrm{NL}} \zeta^{(1) 2}$, where, as explained in Section 7.4 the parameter $a_{\mathrm{NL}}$ specifies the level of primordial non-Gaussianity depending on the particular scenario for the generation of the cosmological perturbations. On the other hand, during radiation-domination

$$
\Phi^{(2)}(0)-\Psi^{(2)}(0)=-Q^{(2)}(0),
$$

where

$$
\begin{aligned}
Q^{(2)}(0)= & -2 \nabla^{-2} \partial_{k} \Phi^{(1)}(0) \partial^{k} \Phi^{(1)}(0) \\
& +6 \frac{\partial_{i} \partial^{j}}{\nabla^{4}}\left(\partial^{i} \Phi^{(1)}(0) \partial_{j} \Phi^{(1)}(0)\right)+\frac{9}{2} \mathcal{H}^{2} \frac{\partial_{i} \partial^{j}}{\nabla^{4}} \Pi_{\gamma j}^{(2) i},
\end{aligned}
$$

where we are evaluating (B.15) in the limit $k \eta \ll 1$. The contribution from the second-order quadrupole moment in this limit reads

$$
\begin{aligned}
& \frac{9}{2} \mathcal{H}^{2} \frac{\partial_{i} \partial^{j}}{\nabla^{4}} \Pi_{\gamma j}^{(2) i} \\
& =\frac{9}{2 \eta^{2}} \frac{8}{3} \frac{\partial_{i} \partial^{j}}{\nabla^{4}}\left(v^{i} v^{j}-\frac{1}{3} \delta_{j}^{i} v^{2}\right) \\
& \equiv-3 \frac{F C}{k^{2} \eta^{2}} \Psi_{\mathbf{k}_{1}}^{(1)}(0) \Psi_{\mathbf{k}_{2}}^{(1)}(0) \sin \left(k_{1} c_{s} \eta\right) \sin \left(k_{2} c_{s} \eta\right) \\
& \longrightarrow \frac{27}{k^{2}} F \Psi_{\mathbf{k}_{1}}^{(1)}(0) \Psi_{\mathbf{k}_{2}}^{(1)}(0),
\end{aligned}
$$

where $F$ and $C$ are defined in (244) and (243). Therefore, we find that in Fourier space

$$
Q^{(2)}(0)=33 \frac{F}{k^{2}} \Psi_{\mathbf{k}_{1}}^{(1)}(0) \Psi_{\mathbf{k}_{2}}^{(1)}(0),
$$

and from (267) we read off the intial condition as (convolution products are understood)

$$
\Psi^{(2)}(0)=\left[-3\left(a_{\mathrm{NL}}-1\right)+11 \frac{F\left(\mathbf{k}_{1}, \mathbf{k}_{2}, \mathbf{k}\right)}{k^{2}}\right] \Psi_{\mathbf{k}_{1}}^{(1)}(0) \Psi_{\mathbf{k}_{2}}^{(1)}(0) .
$$

7.6. Perturbation Modes with $k \ll k_{\text {eq. }}$. Let us consider the photon perturbations which enter the horizon between the equality epoch and the recombination epoch, with wavelengths $\eta_{*}^{-1}<k<\eta_{\mathrm{eq}}^{-1}$. In fact, in order to find some analytical solutions, we will assume that by the time of recombination the universe is matter dominated $\eta_{\mathrm{eq}} \ll$ $\eta_{*}$. In this case, the gravitational potentials are sourced by the dark matter component and their evolution is given in Section B. At linear order, the gravitational potentials remain constant in time, while at second-order they are given by (B.6). In turn, the gravitational potentials act as an external force on the $\mathrm{CMB}$ photons as in the equation (214) describing the $\mathrm{CMB}$ energy density evolution in the tightly coupled regime.
For the regime of interest it proves convenient to use the solution of (214) found in (241). The source functions $\delta_{\Delta}$ and $\delta_{V}^{i}$ are given by (208) and (213), respectively. In particular $\delta_{\Delta}$ at early times- $s_{\Delta}(0)$ appearing in (241) vanishes. For a matter-dominated period

$$
\begin{aligned}
\S_{\Delta}(R=0)= & \left(\Delta_{00}^{(1) 2}\right)^{\prime}-\frac{16}{3} \Psi^{(1)} \partial_{i} v_{\gamma}^{(1) i}+\frac{16}{3}\left(v_{\gamma}^{2}\right)^{\prime} \\
& +\frac{32}{3} \partial^{i} \Psi^{(1)} v_{i},
\end{aligned}
$$

where we have used the linear evolution equations (C.1) and (C.5) with $\Phi^{(1)}=\Psi^{(1)}$, and

$$
\begin{aligned}
S_{V}^{i}(R=0)= & \frac{8}{3} v_{\gamma}^{(1) i} \partial_{j} v_{\gamma}^{(1) j}+\frac{1}{4} \partial^{i} \Delta_{00}^{(1) 2}-2 \partial^{i} \Psi^{(1) 2} \\
& -\Psi^{(1)} \partial^{i} \Delta_{00}^{(1)}-2 \omega^{i^{\prime}}-\frac{3}{4} \partial_{j} \Pi^{(2) i j} .
\end{aligned}
$$

As at linear order, we are evaluating these expressions in the limit $R=3 \rho_{b} / 4 \rho_{\gamma} \rightarrow 0$, while retaining a non-vanishing and constant value for $R$ in the expression for the photonbaryon fluid sound speed entering in the sines and cosines. In fact, this approximation gives the dominant contributions to the source terms $\delta_{\Delta}$ and $\delta_{V}^{i}$ made by first-order squared terms. Using the linear solutions (C.15) and (C.17) for the energy density and velocity of photons, the source functions in Fourier space read

$$
\begin{aligned}
& \S_{\Delta}(R=0) \\
& =\left[-2\left(\frac{6}{5}\right)^{2} k_{2} c_{s} \cos \left(k_{1} c_{s} \eta\right) \sin \left(k_{2} c_{s} \eta\right)+\frac{108}{25} k_{2} c_{s}\right. \\
& \times \sin \left(k_{2} c_{s} \eta\right)-\frac{32}{3}\left(\frac{9}{10}\right)^{2} \frac{\mathbf{k}_{1}}{k_{1}} \cdot \mathbf{k}_{2} c_{s}^{3} \\
& \times \sin \left(k_{1} c_{s} \eta\right) \cos \left(k_{2} c_{s} \eta\right)+\frac{32}{3}\left(\frac{9}{10}\right)^{2} \\
& \left.\times \frac{\mathbf{k}_{1}}{k_{1}} \cdot \mathbf{k}_{2} c_{s} \sin \left(k_{1} c_{s} \eta\right)\right] \Psi_{\mathbf{k}_{1}}^{(1)}(0) \Psi_{\mathbf{k}_{2}}^{(1)}(0), \\
& \oint_{V}^{i}(R=0) \\
& =\left[-i \frac{2}{3}\left(\frac{9}{10}\right)^{2} c_{s}^{2} \frac{k_{1}^{i}}{k_{1}} k_{2} \sin \left(k_{1} c_{s} \eta\right) \sin \left(k_{2} c_{s} \eta\right)\right. \\
& +\frac{i}{4} k^{i}\left(\frac{6}{5} \cos \left(k_{1} c_{s} \eta\right)-\frac{18}{5}\right)\left(\frac{6}{5} \cos \left(k_{2} c_{s} \eta\right)-\frac{18}{5}\right) \\
& -2 i k^{i}\left(\frac{9}{10}\right)^{2}-i \frac{9}{10} k_{2}^{i}\left(\frac{6}{5} \cos \left(k_{2} c_{s} \eta\right)-\frac{18}{5}\right)-2 \omega^{i^{\prime}} \\
& \left.+i \frac{2}{3}\left(\frac{9}{10}\right)^{2} c_{s}^{2} \frac{\mathbf{k}_{2}}{k_{2}} \cdot \mathbf{k}_{1} \frac{k_{1}^{i}}{k_{1}} \sin \left(k_{1} c_{s} \eta\right) \sin \left(k_{2} c_{s} \eta\right)\right] \\
& \times \Psi_{\mathbf{k}_{1}}^{(1)}(0) \Psi_{\mathbf{k}_{2}}^{(1)}(0) \text {. }
\end{aligned}
$$

In $\delta_{V}^{i}$, we have used the expression (222) for the secondorder quadrupole moment $\Pi_{\gamma}^{(2) i j}$ of the photons in the 
tight coupling limit, with the velocity $v^{(1)}=v_{\gamma}^{(1)}$. Notice that, for the modes crossing the horizon at $\eta>\eta_{\text {eq }}$, we have expressed the gravitational potential during the matter-dominated period in terms of the initial value on superhorizon scales deep in the radiation dominated epoch as $\Psi^{(1)}=9 \Psi^{(1)}(0) / 10$.

As for the second-order gravitational potentials, we have to compute the combination $\Phi^{(2)}+\Psi^{(2)}$ appearing in (241). The gravitational potential $\Psi^{(2)}$ is given by (B.6), while $\Phi^{(2)}$ is given by

$$
\Phi^{(2)}=\Psi^{(2)}-Q^{(2)},
$$

according to the relation (32), where for a matter-dominated period

$$
Q^{(2)}=5 \nabla^{-4} \partial_{i} \partial_{j}\left(\partial^{i} \Psi^{(1)} \partial_{j} \Psi^{(1)}\right)-\frac{5}{3} \nabla^{-2}\left(\partial_{k} \Psi^{(1)} \partial^{k} \Psi^{(1)}\right) .
$$

We thus find

$$
\begin{aligned}
\Phi^{(2)} & +\Psi^{(2)} \\
= & -5 \nabla^{-4} \partial_{i} \partial^{j}\left(\partial^{i} \Psi^{(1)} \partial_{j} \Psi^{(1)}\right) \\
& +\frac{5}{3} \nabla^{-2}\left(\partial_{k} \Psi^{(1)} \partial^{k} \Psi^{(1)}\right)+2 \Psi_{m}^{(2)}(0) \\
& -\frac{1}{7}\left(\partial_{k} \Psi^{(1)} \partial^{k} \Psi^{(1)}-\frac{10}{3} \nabla^{-2} \partial_{i} \partial^{j}\left(\partial^{i} \Psi^{(1)} \partial_{j} \Psi^{(1)}\right)\right) \eta^{2},
\end{aligned}
$$

which in Fourier space reads

$$
\begin{aligned}
& \Phi^{(2)}+\Psi^{(2)} \\
& =2 \Psi_{m}^{(2)}(0)+\left[\frac{1}{7} G\left(\mathbf{k}_{1}, \mathbf{k}_{2}, \mathbf{k}\right) \eta^{2}-\frac{5}{k^{2}} F\left(\mathbf{k}_{1}, \mathbf{k}_{2}, \mathbf{k}\right)\right] \\
& \quad \times\left(\frac{9}{10}\right)^{2} \Psi_{\mathbf{k}_{1}}^{(1)}(0) \Psi_{\mathbf{k}_{2}}^{(1)}(0),
\end{aligned}
$$

where the kernels of the convolutions are given by (245) and

$$
G\left(\mathbf{k}_{1}, \mathbf{k}_{2}, \mathbf{k}\right)=\mathbf{k}_{1} \cdot \mathbf{k}_{2}-\frac{10}{3} \frac{\left(\mathbf{k} \cdot \mathbf{k}_{1}\right)\left(\mathbf{k} \cdot \mathbf{k}_{2}\right)}{k^{2}} .
$$

In (279), $\Psi_{m}^{(2)}(0)$ is the initial condition for the gravitational potential fixed at some time $\eta_{i}>\eta_{\text {eq }}$. For the regime of interest it corresponds to the value of the gravitational potential on superhorizon scales during the matter-dominated epoch.
Notice a property that will be useful later on. By looking at (278) and the explicit solution for $\Psi^{(2)}$ (B.6) which grows as $\eta^{2}$, it is easy to realize that on very small scales, for $k \eta \gg 1$, the two gravitational potentials are equal with

$$
\Phi^{(2)} \simeq \Psi^{(2)}=\Psi_{m}^{(2)}(0)+\frac{1}{14} G\left(\mathbf{k}_{1}, \mathbf{k}_{2}, \mathbf{k}\right) \eta^{2} .
$$

We are now able to compute the integrals entering in the solution (241). The one involving the second-order gravitational potentials is straightforward to compute

$$
\begin{aligned}
& -4 k c_{s} \int_{0}^{\eta} d \eta^{\prime}\left(\Phi^{(2)}+\Psi^{(2)}+R \Phi^{(2)}\right) \sin \left[k c_{s}\left(\eta-\eta^{\prime}\right)\right] \\
& =-8 \Psi_{m}^{(2)}(0)\left(1-\cos \left(k c_{s} \eta\right)\right) \\
& -4\left[-\frac{5 F}{k^{2}}\left(1-\cos \left(k c_{s} \eta\right)\right)+\frac{1}{7 k^{2} c_{s}^{2}}\right. \\
& \left.\quad \times G\left(-2+\left(k c_{s} \eta\right)^{2}+2 \cos \left(k c_{s} \eta\right)\right)\right]
\end{aligned}
$$

$$
\begin{aligned}
& \times\left(\frac{9}{10}\right)^{2} \Psi_{\mathbf{k}_{1}}^{(1)}(0) \Psi_{\mathbf{k}_{2}}^{(1)}(0)-4 R \Psi_{m}^{(2)}(0)\left(1-\cos \left(k c_{s} \eta\right)\right) \\
& -4 R\left[-\frac{5 F}{k^{2}}\left(1-\cos \left(k c_{s} \eta\right)\right)+\frac{1}{14 k^{2} c_{s}^{2}}\right. \\
& \left.\quad \times G\left(-2+\left(k c_{s} \eta\right)^{2}+2 \cos \left(k c_{s} \eta\right)\right)\right] \\
& \times\left(\frac{9}{10}\right)^{2} \Psi_{\mathbf{k}_{1}}^{(1)}(0) \Psi_{\mathbf{k}_{2}}^{(1)}(0),
\end{aligned}
$$

where for brevity $F$ and $G$ stand for the convolutions (245) and (281). Notice also the we have isolated the terms proportional to $R$ as it will be useful later on.

For the two remaining integrals, in the following we will show only the terms that in the final expression for $\Delta_{00}^{(2)}$ and the second-order velocity $v_{\gamma}^{(2) i}$ give the dominant contributions for $k \eta \gg 1$, even though we have perfomed a fully computation. The integral over the source function $\varsigma_{\Delta}$ yields a sum of oscillating functions (cosines) which turn out to be subdominant, so we do not display the full result. For the last integral we find $((1 \leftrightarrow 2)$ stands by an exchange of indices)

$$
\begin{aligned}
- & \frac{4}{3} \frac{i k_{i}}{k c_{s}} \int_{0}^{\eta} d \eta^{\prime} s_{V}^{i} \sin \left[k c_{s}\left(\eta-\eta^{\prime}\right)\right] \\
& =\left[\frac{27}{25} \frac{2 \mathbf{k} \cdot \mathbf{k}_{2}+k^{2}}{k^{2} c_{s}^{2}}+(1 \longleftrightarrow 2)\right] \Psi_{\mathbf{k}_{1}}^{(1)}(0) \Psi_{\mathbf{k}_{2}}^{(1)}(0),
\end{aligned}
$$

where the terms that have been dropped vary in time as sines and cosines. We have written the contribution in (284) because, upon integration over time, it will give a nonnegligible contribution to the velocity $v_{\gamma}^{(2) i}$. 
From the general solution (241) and the expression (B.6) for the second-order gravitational potential $\Psi^{(2)}$, we thus obtain

$$
\begin{aligned}
\Delta_{00}^{(2)}= & -4(1+R) \Psi_{m}^{(2)}(0) \\
& +\left[2\left(9 a_{\mathrm{NL}}-7\right) \Psi_{\mathbf{k}_{1}}^{(1)}(0) \Psi_{\mathbf{k}_{2}}^{(1)}(0)+(8+4 R) \Psi_{m}^{(2)}(0)\right] \\
& \times \cos \left(k c_{s} \eta\right)-\frac{2}{7} G\left(\mathbf{k}_{1}, \mathbf{k}_{2}, \mathbf{k}\right) \eta^{2}(1+R)\left(\frac{9}{10}\right)^{2} \\
& \times \Psi_{\mathbf{k}_{1}}^{(1)}(0) \Psi_{\mathbf{k}_{2}}^{(1)}(0) .
\end{aligned}
$$

We warn the reader that in writing (285), we have kept all those terms that contain the primordial non-Gaussianity parametrized by $a_{\mathrm{NL}}$, and the terms which dominate at late times for $k \eta \gg 1$.

7.6.1. Initial Conditions for the Second-Order Gravitational Potentials. The initial condition $\Psi_{m}^{(2)}(0)$ for the modes that cross the horizon after the equality epoch is fixed by the value of the gravitational potential on superhorizon scales during the matter dominated epoch. To compute this value we use the conservation on superhorizon scales of the curvature perturbation $\zeta^{(2)}$ defined in (36). For a matter-dominated period, the curvature perturbation on large-scales turns out to be

$$
\zeta^{(2)}=-\Psi_{m}^{(2)}(0)+\frac{1}{3} \frac{\delta^{(2)} \rho_{m}}{\rho_{m}}+\frac{38}{9} \Psi_{m}^{(1) 2}(0)
$$

where we used the energy continuity equation $\delta^{(1)} \rho_{m}^{\prime}+$ $3 \mathscr{H} \delta^{(1)} \rho_{m}-3 \rho_{m} \Psi^{(1)^{\prime}}=0$ and the $(0-0)$ Einstein equation $\delta^{(1)} \rho_{m} / \rho_{m}=-2 \Psi^{(1)}$ in the superhorizon limit.

From the $(0-0)$ Einstein equation on large scales $\delta^{(2)} \rho_{m} / \rho_{m}=-2 \Phi^{(2)}+4 \Phi^{(1) 2}$ bringing

$$
\zeta^{(2)}=-\frac{5}{3} \Psi_{m}^{(2)}(0)-\frac{2}{3}\left(\Phi_{m}^{(2)}(0)-\Psi_{m}^{(2)}(0)\right)+\frac{50}{9} \Psi_{m}^{(1) 2}(0)
$$

The conserved value of $\zeta^{(2)}$ is parametrized as in (38), $\zeta^{(2)}=$ $2 a_{\mathrm{NL}} \zeta^{(1) 2}=\left(50 a_{\mathrm{NL}} / 9\right) \Psi^{(1) 2}$, with $\zeta^{(1)}=-5 \Psi^{(1)} / 3$ on large scales after the equality epoch. At second order, the two gravitational potentials in a matter dominated epoch differ according to (278) and using (287), we find

$$
\begin{aligned}
\Psi_{m}^{(2)}(0)= & -\frac{27}{10}\left(a_{\mathrm{NL}}-1\right)\left(\Psi^{(1)}(0)\right)^{2}+\left(\frac{9}{10}\right)^{2} \\
& \times\left[2 \nabla^{-4} \partial_{i} \partial^{j}\left(\partial^{i} \Psi^{(1)}(0) \partial_{j} \Psi^{(1)}(0)\right)\right. \\
& \left.-\frac{2}{3} \nabla^{-2}\left(\partial_{k} \Psi^{(1)}(0) \partial^{k} \Psi^{(1)}(0)\right)\right],
\end{aligned}
$$

we have expressed the gravitational potential during the matter dominated period $\Psi^{(1)}$ in terms of the initial value on superhorizon scales after the equality epoch as $\Psi^{(1)}=$ $9 \Psi^{(1)}(0) / 10$. In Fourier space, (288) becomes

$$
\begin{aligned}
\Psi_{m}^{(2)}(0)= & {\left[-\frac{27}{10}\left(a_{\mathrm{NL}}-1\right)+2\left(\frac{9}{10}\right)^{2} \frac{F\left(\mathbf{k}_{1}, \mathbf{k}_{2}, \mathbf{k}\right)}{k^{2}}\right] } \\
& \times \Psi_{\mathbf{k}_{1}}^{(1)}(0) \Psi_{\mathbf{k}_{2}}^{(1)}(0),
\end{aligned}
$$

where $F$ is the kernel defined in (245).

We can use the explicit expression for $\Psi_{m}^{(2)}(0)$ in (285), still keeping only the terms that contain the primordial non-Gaussianity parametrized by $a_{\mathrm{NL}}$, and the terms which dominate at late times for $k \eta \gg 1$ to find

$$
\begin{aligned}
\Delta_{00}^{(2)}=[ & \frac{54}{5}(1+R)\left(a_{\mathrm{NL}}-1\right)-\frac{2}{5}\left(9 a_{\mathrm{NL}}-19\right) \\
& \times \cos \left(k c_{s} \eta\right)+\frac{54}{5} R\left(a_{\mathrm{NL}}-1\right) \cos \left(k c_{s} \eta\right) \\
& \left.-\frac{2}{7}\left(\frac{9}{10}\right)^{2} G\left(\mathbf{k}_{1}, \mathbf{k}_{2}, \mathbf{k}\right) \eta^{2}(1+R)\right] \\
& \times \Psi_{\mathbf{k}_{1}}^{(1)}(0) \Psi_{\mathbf{k}_{2}}^{(1)}(0) .
\end{aligned}
$$

7.6.2. Second-Order Photon Velocity Perturbation. The second-order velocity of the photons can be obtained from (212)

$$
v_{\gamma}^{(2) i} \simeq \int_{0}^{\eta} d \eta^{\prime}\left(s_{V}^{i}-\partial^{i} \Phi^{(2)}-\frac{1}{4} \partial^{i} \Delta_{00}^{(2)}\right) .
$$

Notice that for simplicity in writing (291) we have dropped off the dependence on $R$. In fact, the main conclusions of this Subsection remains unchanged. The second-order gravitional potential in matter-dominated universe can be obtained from (277)-(278) and (B.6) as

$$
\begin{aligned}
\Phi^{(2)}= & \Psi_{m}^{(2)}(0)-\frac{1}{14}\left(\partial_{k} \Psi^{(1)} \partial^{k} \Psi^{(1)}-\frac{10}{3} \nabla^{-2} \partial_{i} \partial^{j}\right. \\
& \left.\times\left(\partial^{i} \Psi^{(1)} \partial_{j} \Psi^{(1)}\right)\right) \eta^{2}-5 \nabla^{-4} \partial_{i} \partial^{j} \\
& \times\left(\partial^{i} \Psi^{(1)} \partial_{j} \Psi^{(1)}\right)+\frac{5}{3} \nabla^{-2}\left(\partial_{k} \Psi^{(1)} \partial^{k} \Psi^{(1)}\right) .
\end{aligned}
$$

In Fourier space, this becomes

$$
\begin{aligned}
\Phi^{(2)}= & \Psi_{m}^{(2)}(0)+\left[\frac{1}{14} G\left(\mathbf{k}_{1}, \mathbf{k}_{2}, \mathbf{k}\right) \eta^{2}-\frac{5}{k^{2}} F\left(\mathbf{k}_{1}, \mathbf{k}_{2}, \mathbf{k}\right)\right] \\
& \times\left(\frac{9}{10}\right)^{2} \Psi_{\mathbf{k}_{1}}^{(1)}(0) \Psi_{\mathbf{k}_{2}}^{(1)}(0),
\end{aligned}
$$


where the kernels of the convolutions are given by (245) and (281). The integral over $\Phi^{(2)}$ in (291) is then easily computed

$$
\begin{aligned}
-\int_{0}^{\eta} d \eta^{\prime} \partial^{i} \Phi^{(2)} & \\
\equiv-i k^{i}[ & \Psi_{m}^{(2)}(0) \eta \\
& +\left(\frac{1}{42} G\left(\mathbf{k}_{1}, \mathbf{k}_{2}, \mathbf{k}\right) \eta^{3}-\frac{5}{k^{2}} F\left(\mathbf{k}_{1}, \mathbf{k}_{2}, \mathbf{k}\right) \eta\right) \\
& \left.\times\left(\frac{9}{10}\right)^{2} \Psi_{\mathbf{k}_{1}}^{(1)}(0) \Psi_{\mathbf{k}_{2}}^{(1)}(0)\right],
\end{aligned}
$$

where as usual the equivalence symbol means that we are evaluating a given expression in Fourier space. For the integral over the source function $\delta_{V}^{i}$ we use its expression in Fourier space, (276), and the dominant terms for $k \eta \gg 1$ are

$$
\begin{aligned}
& \int_{0}^{\eta} d \eta^{\prime} 8_{V}^{i} \\
& \equiv\left[-2 i k^{i}\left(\frac{9}{10}\right)^{2}+i k^{i} \frac{81}{25}+i \frac{8}{3}\left(\frac{9}{10}\right)^{2} \frac{1}{k^{4}}\left(k_{2}^{2}-k_{1}^{2}\right) \mathbf{k} \cdot \mathbf{k}_{1} k_{2}^{i}\right] \eta .
\end{aligned}
$$

Notice that, in order to compute this integral, we must know the second-order vector metric petturbation $\omega^{i}$. This is easily obtained for a matter-dominated universe from (B.7). Using (B.4) and (B.2) one finds

$$
\begin{aligned}
\omega^{i}= & -\frac{4}{3}\left(\frac{9}{10}\right)^{2} \nabla^{-4} \partial_{j} \\
& \times\left[\partial^{i} \nabla^{2} \Psi^{(1)}(0) \partial^{j} \Psi^{(1)}(0)-\partial^{j} \nabla^{2} \Psi^{(1)}(0) \partial^{i} \Psi^{(1)}(0)\right] \eta,
\end{aligned}
$$

giving rise to the third term in (295).

Finally, for the integral over $\Delta_{00}^{(2)}$ some caution is needed. Since in the final expression for $v_{\gamma}^{(2) i}$ the dominant terms at late times turn out to be proportional $\eta$, one has to use an expression for $\Delta_{00}^{(2)}$ that keep track of all those contributions that, upon integration, scale like $\eta$. Thus, we must use the expression written in (285), plus (284), and some terms of (283) that have been previously neglected in (285). Then, we find for $k \eta \gg 1$

$$
\begin{aligned}
& +\frac{1}{4} \int_{0}^{\eta} d \eta^{\prime} \partial^{i} \Delta_{00}^{(2)} \\
& \equiv \frac{i k^{i}}{4}\left[-4 \Psi_{m}^{(2)}(0) \eta+\left(2\left(9 a_{\mathrm{NL}}-7\right) \Psi_{\mathbf{k}_{1}}^{(1)}(0) \Psi_{\mathbf{k}_{2}}^{(1)}(0)\right.\right. \\
& \left.\left.+8 \Psi_{m}^{(2)}(0)\right) \frac{\sin \left(k c_{s} \eta\right)}{k c_{s}}\right] \\
& +\frac{i k^{i}}{4}\left[-\frac{2}{21}\left(\frac{9}{10}\right)^{2} G \eta^{3}\right. \\
& +\left(\frac{20}{3 c_{s}^{2}}\left(\frac{9}{10}\right)^{2} \frac{F}{k^{2}}+\frac{8}{21 c_{s}^{4}}\left(\frac{9}{10}\right)^{2} \frac{G}{k^{2}}\right. \\
& \left.\left.+\frac{54}{25 c_{s}^{2}} \frac{k^{2}+\mathbf{k} \cdot\left(\mathbf{k}_{1}+\mathbf{k}_{2}\right)}{k^{2}}\right) \eta\right] \Psi_{\mathbf{k}_{1}}^{(1)}(0) \Psi_{\mathbf{k}_{2}}^{(1)}(0)
\end{aligned}
$$

Using (294), (295), and (297) we get

$$
\begin{aligned}
v_{\gamma}^{(2) i}=[i & \frac{k^{i}}{k} \frac{1}{10 c_{s}}\left(9 a_{\mathrm{NL}}-19\right) \sin \left(k c_{s} \eta\right) \\
& +\left(-i \frac{2}{21 c_{s}^{4}} k^{i}\left(\frac{9}{10}\right)^{2} \frac{G}{k^{2}}-2 i\left(\frac{9}{10}\right)^{2} k^{i}\right. \\
& +i \frac{81}{50}\left(k_{2}^{i}+k_{1}^{i}\right)-i \frac{27}{50 c_{s}^{2}} \frac{k^{2}+\mathbf{k} \cdot\left(\mathbf{k}_{1}+\mathbf{k}_{2}\right)}{k^{2}} k^{i} \\
& \left.\left.+i \frac{4}{3}\left(\frac{9}{10}\right)^{2} \frac{k_{2}^{2}-k_{1}^{2}}{k^{4}}\left(\mathbf{k} \cdot \mathbf{k}_{1} k_{2}^{i}-\mathbf{k} \cdot \mathbf{k}_{2} k_{1}^{i}\right)\right) \eta\right] \\
& \times \Psi_{\mathbf{k}_{1}}^{(1)}(0) \Psi_{\mathbf{k}_{2}}^{(1)}(0) .
\end{aligned}
$$

To obtain (298), we have also used the explicit expression (289) for $\Psi_{m}^{(2)}(0)$ and we have kept the terms depending on $a_{\mathrm{NL}}$ parametrizing the primordial non-Gaussianity and the terms that dominate at late times for $k \eta \gg 1$.

7.7. Perturbation Modes with $k \gg k_{\mathrm{eq}}$ : Cold Dark Matter Perturbations at Second Order and Improved Analytical Solutions for CMB Anisotropies. In Section 7.5, we have computed the perturbations of the CBM photons at last scattering for the modes that cross the horizon at $\eta<\eta_{\mathrm{eq}}$ under the approximation that the universe is radiation-dominated. However, around the equality epoch, through recombination, the dark matter component will start to dominate. In this section, we will account for its contribution to the gravitational potential and for the resulting perturbations of the photons from the equality epoch onwards. This leads to a more realistic and accurate analytical solutions for the acoustic oscillations of the photon-baryon fluid for the modes of interest.

The starting point is to consider the density perturbation in the dark matter component for subhorizon modes during the radiation dominated epoch. Its value at the equality epoch will fix the magnitude of the gravitational potential at $\eta_{\mathrm{eq}}$ and hence the initial conditions for the subsequent evolution of the photons fluctuations during the matter dominated period. At linear order, the procedure is standard (see, e.g [70]), and we will use a similar one at second-order in the perturbations. Thus, this section serves also as a guide through the evolution of the CDM density perturbations at second-order accounting for those modes that enter the horizon during the radiation dominated epoch. This allows to determine the second-order transfer function for the density perturbations with a generalization at second-orde of the Meszaros effect.

7.7.1. Subhorizon Evolution of CDM Perturbations for $\eta<\eta_{\mathrm{eq}}$. From the energy and velocity continuity equations for CDM, it is possible to isolate an evolution equation for the density perturbation $\delta_{d}=\delta \rho_{d} / \rho_{d}$, where the subscript $d$ stands for cold dark matter. In [46], we have obtained the Boltzmann 
equations up to second-order for CDM. The number density of CDM evolves according to [46]

$$
\begin{aligned}
\frac{\partial n_{d}}{\partial \eta}+ & e^{\Phi+\Psi} \frac{\partial\left(v_{d}^{i} n_{d}\right)}{\partial x^{i}}+3\left(\mathcal{H}-\Psi^{\prime}\right) n_{d}-2 e^{\Phi+\Psi} \Psi_{, k} v_{d}^{k} n_{d} \\
& +e^{\Phi+\Psi} \Phi_{, k} v_{d}^{k} n_{d}=0 .
\end{aligned}
$$

At linear order, $n_{d}=\bar{n}_{d}+\delta^{(1)} n_{d}$ and one recovers the usual energy continuity equation

$$
\delta_{d}^{(1)^{\prime}}+v_{d, i}^{(1) i}-3 \Psi^{(1), i}=0,
$$

with $\delta_{d}^{(1)}=\delta^{(1)} \rho_{d} / \bar{\rho}_{d}=\delta^{(1)} n_{d} / \bar{n}_{d}$. The CDM velocity at the same order of perturbation obeys [46]

$$
v_{d}^{(1) i^{\prime}}+\mathscr{H} v_{d}^{(1) i}=-\Phi^{(1), i} .
$$

Perturbing $n_{\mathrm{CDM}}$ up to second-order, we find

$$
\begin{aligned}
\delta_{d}^{(2)^{\prime}}+ & v_{d, i}^{(2) i}-3 \Psi^{(2)^{\prime}} \\
= & -2\left(\Phi^{(1)}+\Psi^{(1)}\right) v_{d, i}^{(1) i}-2 v_{d, i}^{(1) i} \delta_{d}^{(1)}-2 v_{d}^{(1) i} \delta_{d, i}^{(1)} \\
& +6 \Psi^{(1)^{\prime}} \delta_{d}^{(1)}+\left(4 \Psi_{, k}^{(1)}-2 \Phi_{, k}^{(1)}\right) v_{d}^{(1) k} .
\end{aligned}
$$

The R.H.S. of this equation can be further manipulated by using the linear equation (300) to replace $v_{d, i}^{(1) i}$ yielding

$$
\begin{aligned}
\delta_{d}^{(2)^{\prime}} & +v_{d, i}^{(2) i}-3 \Psi^{(2)^{\prime}} \\
= & 4 \delta_{d}^{(1)^{\prime}} \Psi^{(1)}-6\left(\Psi^{(1) 2}\right)^{\prime}+\left(\delta_{d}^{(1) 2}\right)^{\prime} \\
& -2 v_{d}^{(1) i} \delta_{d, i}^{(1)}+2 \Psi_{, k}^{(1)} v_{d}^{(1) k},
\end{aligned}
$$

where we use $\Phi^{(1)}=\Psi^{(1)}$. In [46] the evolution equation for the second-order CDM velocity perturbation has been already obtained

$$
\begin{aligned}
v_{d}^{(2) i^{\prime}} & +\mathscr{H} v_{d}^{(2) i}+2 \omega^{i^{\prime}}+2 \mathcal{H} \omega^{i}+\Phi^{(2), i} \\
& =2 \Psi^{(1)^{\prime}} v_{d}^{(1) i}-2 v_{d}^{(1) j} \partial_{j} v_{d}^{(1) i}-4 \Phi^{(1)} \Phi^{(1), i} .
\end{aligned}
$$

At linear order, we can take the divergence of (301) and, using (300) to replace the velocity perturbation, we obtain a differental equation for the CDM density contrast

$$
\left[a\left(3 \Psi^{(1)^{\prime}}-\delta_{d}^{(1)^{\prime}}\right)\right]^{\prime}=-a \nabla^{2} \Phi^{(1)},
$$

which can be rewritten as

$$
\delta_{d}^{(1)^{\prime \prime}}+\mathscr{H} \delta_{d}^{(1)^{\prime}}=S^{(1)},
$$

where

$$
S^{(1)}=3 \Psi^{(1)^{\prime \prime}}+3 \mathscr{H} \Psi^{(1)^{\prime}}+\nabla^{2} \Phi^{(1)} .
$$

When the radiation is dominating the gravitational potential is mainly due to the perturbations in the photons, and $a(\eta) \propto \eta$. For subhorizon scales, (306) can be solved following the procedure introduced in [74]. Using the Green method, the general solution to (306) (in Fourier space) is given by

$$
\begin{aligned}
\delta_{d}^{(1)}(\mathbf{k}, \eta)= & C_{1}+C_{2} \ln (\eta) \\
& -\int_{0}^{\eta} d \eta^{\prime} S^{(1)}\left(\eta^{\prime}\right) \eta^{\prime}\left(\ln \left(k \eta^{\prime}\right)-\ln (k \eta)\right),
\end{aligned}
$$

where the first two terms correspond to the solution of the homogeneous equation. At early times, the density contrast is constant with

$$
\delta_{d}^{(1)}(0)=\frac{3}{4} \Delta_{00}^{(1)}(0)=-\frac{3}{2} \Phi_{\mathbf{k}}^{(1)}(0),
$$

having used the adiabaticity condition, and thus we fix the integration constant as

$$
C_{1}=-\frac{3 \Phi_{\mathrm{k}}^{(1)}(0)}{2},
$$

and $C_{2}=0$. The gravitational potential during the radiationdominated epoch starts to decay as a given mode enters the horizon. Therefore, the source term $S^{(1)}$ behaves in a similar manner and this implies that the integrals over $\eta^{\prime}$ reach asymptotically a constant value. Once the mode has crossed the horizon we can thus write the solution as

$$
\delta_{d}^{(1)}(\mathbf{k}, \eta)=A^{(1)} \Phi^{(1)}(0) \ln \left[B^{(1)} k \eta\right],
$$

where the constants $A^{(1)}$ and $B^{(1)}$ are defined as

$$
A^{(1)} \Phi^{(1)}(0)=\int_{0}^{\infty} d \eta^{\prime} S^{(1)}\left(\eta^{\prime}\right) \eta^{\prime}
$$

$$
A^{(1)} \Phi^{(0)} \ln \left(B^{(1)}\right)=-\frac{3}{2} \Phi^{(1)}(0)-\int_{0}^{\infty} d \eta^{\prime} S^{(1)}\left(\eta^{\prime}\right) \eta^{\prime} \ln \left(k \eta^{\prime}\right) .
$$

The upper limit of the integrals can be taken to infinity because the main contribution comes form when $k \eta \sim 1$ and once the mode has entered the horizon the result will change by a very small quantity. Performing the integrals in (312) and (313), one finds that $A^{(1)}=-9.6$ and $B^{(1)} \simeq 0.44$.

Before moving to the second-order case, a useful quantity to compute is the CDM velocity in a radiation-dominated epoch. From (301), it is given by

$$
\begin{aligned}
v_{d}^{(1) i} & -\frac{1}{a} \int_{0}^{\eta} d \eta^{\prime} \partial^{i} \Phi^{(1)} a\left(\eta^{\prime}\right) \\
& \equiv-3\left(i k^{i}\right) \Phi^{(1)}(0) \frac{k c_{s} \eta-\sin \left(k c_{s} \eta\right)}{k^{3} c_{s}^{3} \eta^{2}},
\end{aligned}
$$

where the last equality holds in Fourier space, and we have used (B.11) (and the fact that $a(\eta) \propto \eta$ when radiation dominates). 
Combining (303) and (304), we get the analogue of (306) at second-order in perturbation theory

$$
\delta_{d}^{(2)^{\prime \prime}}+\mathscr{H} \delta_{d}^{(2)^{\prime}}=S^{(2)},
$$

where the source function is

$$
\begin{gathered}
S^{(2)}=3 \Psi^{(2)^{\prime \prime}}+3 \mathcal{H} \Psi^{(2)^{\prime}}+\nabla^{2} \Phi^{(2)}-2 \partial_{i}\left(\Psi^{(1)} v_{d}^{(1) i}\right) \\
+\nabla^{2} v_{d}^{(1) 2}+2 \nabla^{2} \Phi^{(1) 2} \\
+\frac{1}{a}\left[a \left(4 \delta_{d}^{(1)} \Psi^{(1)}-6\left(\Psi^{(1) 2}\right)^{\prime}+\left(\delta_{d}^{(1) 2}\right)^{\prime}\right.\right. \\
\left.\left.-2 v_{d}^{(1) i} \delta_{d, i}^{(1)}+2 \Psi_{, k}^{(1)} v_{d}^{(1) k}\right)\right]^{\prime} .
\end{gathered}
$$

In fact, we write (315) in a more convenient way as

$$
\delta_{d}^{(2)^{\prime \prime}}-3 \Psi^{(2)^{\prime \prime}}-s_{1}^{\prime}+\mathcal{H}\left(\delta_{d}^{(2)^{\prime}}-3 \Psi^{(2)^{\prime}}-s_{1}\right)=s_{2},
$$

where for simplicity, we have introduced the two functions

$$
\begin{aligned}
s_{1}= & 4 \delta_{d}^{(1)^{\prime}} \Psi^{(1)}-6\left(\Psi^{(1) 2}\right)^{\prime}+\left(\delta_{d}^{(1) 2}\right)^{\prime} \\
& -2 v_{d}^{(1) i} \delta_{d, i}^{(1)}+2 \Psi_{, k}^{(1)} v_{d}^{(1) k} \\
s_{2}= & \nabla^{2} \Phi^{(2)}-2 \partial_{i}\left(\Psi^{(1)^{\prime}} v_{d}^{(1) i}\right) \\
& +\nabla^{2} v_{d}^{(1) 2}+2 \nabla^{2} \Phi^{(1) 2} .
\end{aligned}
$$

In this way, we get an equation of the same form as (306) in the variable $\left[\delta^{(2)}-3 \Psi^{(2)}-\int_{0}^{\eta} d \eta^{\prime} s_{1}\left(\eta^{\prime}\right)\right]$ with source $s_{2}$ on the R.H.S.. Its solution in Fourier space, therefore, is just as (308)

$$
\begin{aligned}
\delta_{d}^{(2)} & -3 \Psi^{(2)}-\int_{0}^{\eta} d \eta^{\prime} s_{1}\left(\eta^{\prime}\right) \\
= & C_{1}+C_{2} \ln (\eta) \\
& -\int_{0}^{\eta} d \eta^{\prime} s_{2}\left(\eta^{\prime}\right) \eta^{\prime}\left[\ln \left(k \eta^{\prime}\right)-\ln (k \eta)\right] .
\end{aligned}
$$

As we will see, (320) provides the generalization of the Meszaros effect at second order in perturbation theory.

7.7.2. Initial Conditions. In the next two sections, we will compute explicitly the expression (320) for the secondorder CDM density contrast on subhorizon scales during the radiation dominate era. First, let us fix the constants $C_{1}$ and $C_{2}$ by appealing to the initial conditions. At $\eta \rightarrow 0$ the L.H.S. of (320) is constant, as one can check by using the results of Section 7.5.2 and the condition of adiabaticity at second-order (see, e.g., $[5,75]$ ) which relates the CDM density contrast at early times on superhorizon scales to the energy density fluctuations of photons by

$$
\begin{aligned}
\delta_{d}^{(2)}(0) & =\frac{3}{4} \Delta_{00}^{(2)}(0)-\frac{1}{3}\left(\delta_{d}^{(1)}(0)\right)^{2} \\
& =\frac{3}{4} \Delta_{00}^{(2)}(0)-\frac{3}{4}\left(\Phi^{(1)}(0)\right)^{2},
\end{aligned}
$$

where in the last step we have used (309). Therefore, we can fix $C_{2}=0$ and

$$
C_{1}=\delta_{d}^{(2)}(0)-3 \Psi^{(2)}(0) .
$$

Equation $(240)$ gives $\Delta_{00}^{(2)}(0)-4 \Psi^{(2)}(0)$ in terms of the primordial non-Gaussianity parametrized by $a_{\mathrm{NL}}$, and the expression for $\Psi^{(2)}(0)$ have been already computed in (272). Thus, we find (in Fourier space)

$$
\Delta_{00}^{(2)}=\left[2\left(3 a_{\mathrm{NL}}-1\right)+44 \frac{F\left(\mathbf{k}_{1}, \mathbf{k}_{2}, \mathbf{k}\right)}{k^{2}}\right] \Psi_{\mathbf{k}_{1}}^{(1)}(0) \Psi_{\mathbf{k}_{2}}^{(1)}(0),
$$

and from (321) we derive the initial density contrast for $\mathrm{CDM}$ at second-order

$$
\delta_{d}^{(2)}(0)=\left[\frac{3}{2}\left(3 a_{\mathrm{NL}}-1\right)+33 \frac{F\left(\mathbf{k}_{1}, \mathbf{k}_{2}, \mathbf{k}\right)}{k^{2}}-\frac{3}{4}\right] \Psi_{\mathbf{k}_{1}}^{(1)}(0) \Psi_{\mathbf{k}_{2}}^{(1)}(0) .
$$

Equation (324) togheter with (272) allows to compute the constant $C_{1}$ as

$$
\begin{aligned}
C_{1} & =\delta_{d}^{(2)}(0)-3 \Psi^{(2)}(0) \\
& =\left[\frac{27}{2}\left(a_{\mathrm{NL}}-1\right)+\frac{9}{4}\right] \Psi_{\mathbf{k}_{1}}^{(1)}(0) \Psi_{\mathbf{k}_{2}}^{(1)}(0) .
\end{aligned}
$$

7.7.3. Meszaros Effect at Second Order. We now compute the integrals over the functions $s_{1}$ and $s_{2}$ appearing in (320). Let us first focus on the integral $\int_{0}^{\eta} d \eta^{\prime} s_{1}\left(\eta^{\prime}\right)$.

Notice that using the linear (300) and (301) for the CDM density and velocity perturbations, the function $s_{1}\left(\eta^{\prime}\right)$ can be written in a more convenient way as

$$
s_{1}(\eta)=-6 \Psi^{(1)} v_{d, i}^{(1) i}+\left(\delta_{d}^{(1) 2}\right)^{\prime}-2 v_{d}^{(1) i} \delta_{d, i}^{(1)}+2\left(\Psi^{(1)} v_{d}^{(1) k}\right)_{, k},
$$

and then

$$
\begin{aligned}
& \int_{0}^{\eta} d \eta^{\prime} s_{1}\left(\eta^{\prime}\right) \\
& =\left(\delta_{d}^{(1)}(\eta)\right)^{2}-\left(\delta_{d}^{(1)}(0)\right)^{2} \\
& \quad+\int_{0}^{\eta} d \eta^{\prime}\left[-2 v_{d}^{(1) i} \delta_{d, i}^{(1)}+2\left(\Psi^{(1)} v_{d}^{(1) k}\right)_{, k}-6 \Psi^{(1)} v_{d, i}^{(1) i}\right] .
\end{aligned}
$$

In (327), all the quantities are known being first-order perturbations: the linear gravitional potential $\Psi^{(1)}$ for a radiation dominated era is given in (B.11), the CDM velocity perturbation corresponds to (314) and the CDM density contrast is given by (311). Thus, the integral in (327) reads (in Fourier space)

$$
\begin{aligned}
\int_{0}^{\eta} d \eta^{\prime}[- & 3 A^{(1)} \mathbf{k}_{1} \cdot \mathbf{k}_{2} \frac{k_{1} c_{s} \eta^{\prime}-\sin \left(k_{1} c_{s} \eta^{\prime}\right)}{k_{1}^{3} c_{s}^{3} \eta^{\prime 2}} \ln \left(B^{(1)} k_{2} \eta^{\prime}\right) \\
& +\left(9\left(\mathbf{k} \cdot \mathbf{k}_{1}\right)-27 k_{1}^{2}\right) \frac{k_{1} c_{s} \eta^{\prime}-\sin \left(k_{1} c_{s} \eta^{\prime}\right)}{k_{1}^{3} c_{s}^{3} \eta^{\prime 2}} \\
& \left.\times \frac{\sin \left(k_{s} c_{s} \eta^{\prime}\right)-k_{2} c_{s} \eta^{\prime} \cos \left(k_{2} c_{s} \eta^{\prime}\right)}{k_{2}^{3} c_{s}^{3} \eta^{\prime 3}}\right] \Psi_{\mathbf{k}_{1}}^{(1)}(0) \Psi_{\mathbf{k}_{2}}^{(1)}(0) .
\end{aligned}
$$


Let us recall that we are interested in the evolution of the CDM second-order density contrast on subhorizon scales during the radiation dominated epoch. Therefore, once we compute the integrals we are interested in the limit of their expression for late times $(k \eta \gg 1)$. For the first contribution to (328), we find that at late times it is well approximated by the expression

$$
\begin{gathered}
\int_{0}^{\eta} d \eta^{\prime} 3 A^{(1)}\left(\mathbf{k}_{1} \cdot \mathbf{k}_{2}\right) \frac{k_{1} c_{s} \eta^{\prime}-\sin \left(k_{1} c_{s} \eta^{\prime}\right)}{k_{1}^{3} c_{s}^{3} \eta^{\prime 2}} \ln \left(B^{(1)} k_{2} \eta^{\prime}\right) \\
\simeq 3 A^{(1)} \frac{\mathbf{k}_{1} \cdot \mathbf{k}_{2}}{k_{1}^{2} c_{s}^{2}}\left[2 . 2 \left(-\frac{1.2}{2}\left[\ln \left(k_{1} c_{s} \eta\right)\right]^{2}\right.\right. \\
\left.\left.+\ln \left(B^{(1)} k_{2} \eta\right) \ln \left(k_{1} c_{s} \eta\right)\right)\right] .
\end{gathered}
$$

We have computed also the remaining integral in (328), but it turns out to be negligible compared to (329). The reason is that the integrand oscillates with an amplitude decaying in time as $\eta^{-3}$, and thus it leads just to a constant (the argument is the same we used at linear order to compute the integrals in (308)). Thus, we can write

$$
\begin{aligned}
& \int_{0}^{\eta} d \eta^{\prime} s_{1}\left(\eta^{\prime}\right) \\
& =\left(\delta_{d}^{(1)}(\eta)\right)^{2}-\left(\delta_{d}^{(1)}(0)\right)^{2}-3 A^{(1)} \frac{\mathbf{k}_{1} \cdot \mathbf{k}_{2}}{k_{1}^{2} c_{s}^{2}} \\
& \quad \times\left[2.2\left(-\frac{1.2}{2}\left[\ln \left(k_{1} c_{s} \eta\right)\right]^{2}+\ln \left(B^{(1)} k_{2} \eta\right) \ln \left(k_{1} c_{s} \eta\right)\right)\right] \\
& \quad \times \Psi_{\mathbf{k}_{1}}^{(1)}(0) \Psi_{\mathbf{k}_{2}}^{(1)}(0) .
\end{aligned}
$$

We now compute the integrals over the function $s_{2}(\eta)$ given in (319). Since at late times $\phi^{(1) 2} \sim 1 / \eta^{4}$ and $\left(\Psi^{(1)^{\prime}} v_{d}^{(1) i}\right)_{, i} \sim 1 / \eta^{3}$ the main contribution to the integral will come from the two remaining terms, $\Phi^{(2)}$ and $v_{d}^{(1) 2}$, whose amplitudes scale at late times as $1 / \eta^{2}$

$$
s_{2} \simeq \nabla^{2} \Phi^{(2)}+\nabla^{2} v_{d}^{(1) 2}
$$

Two are the integrals that we have to compute

$$
\int_{0}^{\eta} d \eta^{\prime} s_{2}\left(\eta^{\prime}\right) \eta^{\prime} \ln \left(k \eta^{\prime}\right)
$$

and the one multiplying $\ln (k \eta)$

$$
\int_{0}^{\eta} d \eta^{\prime} s_{2}\left(\eta^{\prime}\right) \eta^{\prime}
$$

Let us first consider the contributions from $\nabla^{2} v_{d}^{(1) 2}$. The second integral is easily computed using the expression (314) for the linear CDM velocity. We find that at late times the dominant term is (for $k \eta \gg 1$ )

$$
\begin{aligned}
-\int_{0}^{\eta} d \eta^{\prime} \nabla^{2} v_{d}^{(1) 2} \eta^{\prime} \equiv & -\left[\frac{9}{c_{s}^{4}} k^{2} \frac{\mathbf{k}_{1} \cdot \mathbf{k}_{2}}{k_{1}^{2} k_{2}^{2}} \ln (\mathrm{k} \eta)\right] \\
& \times \Psi_{\mathbf{k}_{1}}^{(1)}(0) \Psi_{\mathbf{k}_{2}}^{(1)}(0) .
\end{aligned}
$$

The first integral can be computed by making the following approximation: we split the integral between $0<k \eta<1$ and $k \eta>1$ and for $0<k \eta<1$, we use the asymptotic expression

$$
v_{d}^{(1) i} \approx-\frac{1}{2} i k^{i} \eta \Psi_{\mathbf{k}}^{(1)}(0) \quad(k \eta \ll 1),
$$

while for $k \eta>1$ we use the limit

$$
v_{d}^{(1) i} \approx-i \frac{3}{c_{s}^{2}} \frac{k^{i}}{k} \frac{1}{k \eta} \Psi_{\mathbf{k}}^{(1)}(0) \quad(k \eta \gg 1) .
$$

The the integral for $0<k \eta<1$ just gives a constant, while the integral for $k \eta>1$ brings the dominant contribution at late times being proportional to $[\ln (k \eta)]^{2}$ so that we can write $(k \eta \gg 1)$

$$
\begin{aligned}
& \int_{0}^{\eta} d \eta^{\prime} \nabla^{2} v_{d}^{(1) 2} \eta^{\prime} \ln \left(k \eta^{\prime}\right) \\
& \quad=\frac{9}{2 c_{s}^{4}} k^{2} \frac{\mathbf{k}_{1} \cdot \mathbf{k}_{2}}{k_{1}^{2} k_{2}^{2}}[\ln (k \eta)]^{2} \Psi_{\mathbf{k}_{1}}^{(1)}(0) \Psi_{\mathbf{k}_{2}}^{(1)}(0) .
\end{aligned}
$$

As far as the contribution to the integrals (332) and (333) due to $\nabla^{2} \Phi^{(2)}$ is concerned we have just to keep track of the initial condition provided by the primordial nonGaussianity. We have verified that all the other terms give a negligible contribution. This is easy to understand: the integrand function on large scale is a constant while at late times it oscillates with decreasing amplitudes as $\eta^{-2}$, and thus the integrals will tend asymptotically to a constant. We find that

$$
\begin{gathered}
\int_{0}^{\eta} d \eta^{\prime} \nabla^{2} \Phi^{(2)} \eta^{\prime} \simeq-9 \Phi^{(2)}(0), \\
\int_{0}^{\eta} d \eta^{\prime} \nabla^{2} \Phi^{(2)} \eta^{\prime} \ln \left(k \eta^{\prime}\right) \simeq\left(-9+9 \gamma-9 \frac{\ln 3}{2}\right) \Phi^{(2)}(0),
\end{gathered}
$$

where $\gamma=0.577 \ldots$ is the Euler constant, and $\Phi^{(2)}(0)$ is given by (268).

Therefore, from (337), (334), and (338) we find that for $k \eta \gg 1$

$$
\begin{aligned}
& \int_{0}^{\eta} d \eta^{\prime} s_{2}\left(\eta^{\prime}\right) \eta^{\prime}\left[\ln \left(k \eta^{\prime}\right)-\ln (k \eta)\right] \\
& =-\frac{9}{2 c_{s}^{4}} k^{2} \frac{\mathbf{k}_{1} \cdot \mathbf{k}_{2}}{k_{1}^{2} k_{2}^{2}}[\ln (k \eta)]^{2} \\
& \quad \times \Psi_{\mathbf{k}_{1}}^{(1)}(0) \Psi_{\mathbf{k}_{2}}^{(1)}(0)+9 \Phi^{(2)} \\
& \quad \times(0)\left(-9+9 \gamma-9 \frac{\ln (3)}{2}\right) \ln (k \eta) \Phi^{(2)}(0) .
\end{aligned}
$$


Let us collect the results of (325), (330), and (339) into (320). We find that for $k \eta \gg 1$

$$
\begin{aligned}
& \delta_{d}^{(2)}(k \eta \gg 1) \\
& =\left[-3\left(a_{\mathrm{NL}}-1\right) A_{1} \ln \left(B_{1} k \eta\right)+A_{1}^{2} \ln \left(B_{1} k_{1} \eta\right) \ln \left(B_{1} k_{2} \eta\right)\right. \\
& +\left[-\frac{3}{2} A_{1} \frac{\mathbf{k}_{1} \cdot \mathbf{k}_{2}}{k_{1}^{2} c_{s}^{2}} 2.2\left(-\frac{1.2}{2}\left[\ln \left(k_{1} c_{s} \eta\right)\right]^{2}\right.\right. \\
& \left.\quad+\ln \left(B_{1} k_{2} \eta\right) \ln \left(k_{1} c_{s} \eta\right)\right) \\
& \left.\quad+(1 \longleftrightarrow 2)]+\frac{9}{2 c_{s}^{4}} k^{2} \frac{\mathbf{k}_{1} \cdot \mathbf{k}_{2}}{k_{1}^{2} k_{2}^{2}}[\ln (k \eta)]^{2}\right] \\
& \quad \times \Psi_{\mathbf{k}_{1}}^{(1)}(0) \Psi_{\mathbf{k}_{2}}^{(1)}(0) .
\end{aligned}
$$

Notice that in (320) we have neglected $\Psi^{(2)}$, which decays on subhorizon scales during the radiation dominated epoch (see (250), and we have used (309) and (311). Equation (340) represents the second-order Meszaros effect: the CDM density contrast on small scales (inside the horizon) slowly grows starting from the initial conditions that, at second-order, are set by the primordial non-Gaussianity parameter $a_{\mathrm{NL}}$. As one could have guessed the primordial non-Gaussianity is just transferred linearly. The other terms scale in time as a logarithm squared. We stress that the computation of these terms allows one to derive the full transfer function for the matter perturbations at second order accounting for the dominant second-order corrections. In the next section, we will use (340) to fix the initial conditions for the evolution on subhorizon scales of the photons density fluctuations $\Delta_{00}^{(2)}$ after the equality epoch.

7.7.4. Second-Order CMB Anisotropies for Modes Crossing the Horizon During the Radiaton Epoch. In this section, we derive the energy density perturbations $\Delta_{00}^{(2)}$ of the photons during the matter dominated epoch, for the modes that cross the horizon before equality. In Section 7.6, we have already solved the problem assuming matter domination for modes crossing the horizon after equality. Thus, it is sufficient to take the result (285) and replace the initial conditions

$$
\begin{aligned}
\Delta_{00}^{(2)}= & -4(1+R) \Psi_{m}^{(2)}(0) \\
& +\left[A+(8+4 R) \Psi_{m}^{(2)}(0)\right] \cos \left(k c_{s} \eta\right)+B \sin \left(k c_{s} \eta\right) \\
& -\frac{2}{7} G\left(\mathbf{k}_{1}, \mathbf{k}_{2}, \mathbf{k}\right) \eta^{2}(1+R)\left(\frac{9}{10}\right)^{2} \Psi_{\mathbf{k}_{1}}^{(1)}(0) \Psi_{\mathbf{k}_{2}}^{(1)}(0) .
\end{aligned}
$$

where we have restored the generic integration constants $A$ and $B, \Psi^{(1)}$ is the linear gravitational potential (which is constant for the matter era) and $\Psi_{m}^{(2)}(0)$ represents the initial condition for the second-order gravitational potential fixed at some time $\eta_{i}>\eta_{\mathrm{eq}}$. Equation (340) allows to fix the proper initial conditions for the gravitational potentials on subhorizon scales (accounting for the fact that around the equality epoch they are mainly determined by the CDM density perturbations). At linear order this is achieved by solving the equation for $\delta_{d}^{(1)}$ which is obtained from (305) and the $(0-0)$ Einstein equation which reads (see (29))

$$
3 \mathscr{H} \Psi^{(1)^{\prime}}+3 \mathscr{H}^{2} \Psi^{(1)}-\nabla^{2} \Psi^{(1)}=-\frac{3}{2} \mathscr{H}^{2}\left(\frac{\rho_{d}}{\rho} \delta_{d}^{(1)}+\frac{\rho_{\gamma}}{\rho} \Delta_{00}^{(1)}\right) .
$$

On small scales one neglects the time derivatives of the gravitational potential in (305) and (342) to obtain

$$
\delta_{d}^{(1)^{\prime \prime}}+\mathscr{H} \delta_{d}^{(1)^{\prime}}=\frac{3}{2} \mathscr{H}^{2} \delta_{d}^{(1)}
$$

where we have also dropped the contribution to the gravitational potential from the radiation component. The solution of this equation is matched to the value that $\delta_{d}^{(1)}$ has during the radiation dominated epoch on subhorizon scales, (311), and one finds that for $\eta \gg \eta_{\mathrm{eq}}$ on subhorizon scales the gravitational potential remains constant with

$$
\Psi_{\mathbf{k}}^{(1)}\left(\eta>\eta_{\mathrm{eq}}\right)=\frac{\ln \left(0.15 k \eta_{\mathrm{eq}}\right)}{\left(0.27 k \eta_{\mathrm{eq}}\right)^{2}} \Psi_{\mathbf{k}}^{(1)}(0)
$$

We skip the details of the derivation of (344) since it is a standard computation that the reader can find, for example, in [70]. Since around $\eta_{\mathrm{eq}}$ the dark matter begins to dominate, an approximation to the result (344) can be simply achieved by requiring that during matter domination the gravitational potential remains constant to a value determined by the density contrast (311) at the equality epoch

$$
\left.\left.\nabla^{2} \Psi^{(1)}\right|_{\eta_{\mathrm{eq}}} \simeq \frac{3}{2} \mathscr{H}^{2} \delta_{d}^{(1)}\right|_{\eta_{\mathrm{eq}}},
$$

from (342) on small scales, leading to

$$
\begin{aligned}
\Psi_{\mathbf{k}}^{(1)}\left(\eta>\eta_{\mathrm{eq}}\right) & \simeq-\left.\frac{6}{\left(k \eta_{\mathrm{eq}}\right)^{2}} \delta_{d}^{(1)}\right|_{\eta_{\mathrm{eq}}} \\
& =\frac{\ln \left(B B_{1} k \eta_{\mathrm{eq}}\right)}{\left(0.13 k \eta_{\mathrm{eq}}\right)^{2}} \Psi_{\mathbf{k}}^{(1)}(0),
\end{aligned}
$$

where we used $a(\eta) \propto \eta^{2}$ during matter domination and (311) with $A_{1}=-9.6$ and $B_{1}=0.44$.

At second order, we follow a similar approximation. The general solution for the evolution of the the second-order gravitational potential $\Psi^{(2)}$ for $\eta>\eta_{\text {eq }}$ is given by (B.6). We have to determine the initial conditions for those modes that 
cross the horizon during the radiation epoch. The $(0-0)$ Einstein equation reads

$$
\begin{aligned}
- & \frac{3}{2} \mathscr{H}^{2}\left(\frac{\rho_{d}}{\rho} \delta_{d}^{(2)}+\frac{\rho_{\gamma}}{\rho} \Delta_{00}^{(2)}\right) \\
= & 3 \mathscr{H} \Psi^{(2)^{\prime}}+3 \mathscr{H}^{2} \Phi^{(2)}-\nabla^{2} \Psi^{(2)} \\
& -6 \mathscr{H}^{2}\left(\Phi^{(1)}\right)^{2}-12 \mathscr{H} \Phi^{(1)} \Psi^{(1)^{\prime}} \\
& -3\left(\Psi^{(1)^{\prime}}\right)^{2}+\partial_{i} \Psi^{(1)} \partial^{i} \Psi^{(1)}-4 \Psi^{(1)} \nabla^{2} \Psi^{(1)} .
\end{aligned}
$$

We fix the initial conditions with the matching at equality (neglecting the radiation component)

$$
\begin{aligned}
& \nabla^{2} \Psi^{(2)}-\partial_{i} \Psi^{(1)} \partial^{i} \Psi^{(1)}+\left.4 \Psi^{(1)} \nabla^{2} \Psi^{(1)}\right|_{\eta_{\mathrm{eq}}} \\
& \left.\simeq \frac{3}{2} \mathscr{H}^{2} \delta_{d}^{(2)}\right|_{\eta_{\mathrm{eq}}},
\end{aligned}
$$

where for small scales we neglected the time derivatives in (347). Using (340) to evaluate $\left.\delta_{d}^{(2)}\right|_{\eta_{\mathrm{eq}}}$ and (344) to evaluate $\Psi_{\mathbf{k}}^{(1)}\left(\eta_{\mathrm{eq}}\right)$, we find in Fourier space

$$
\begin{aligned}
& \Psi^{(2)}\left(\eta_{\mathrm{eq}}\right) \\
& =\left[-3\left(a_{\mathrm{NL}}-1\right) \frac{\ln \left(B_{1} k \eta_{\mathrm{eq}}\right)}{\left(0.13 k \eta_{\mathrm{eq}}\right)^{2}}+\left(\frac{\mathbf{k}_{1} \cdot \mathbf{k}_{2}}{k^{2}}-4\right)\right. \\
& \times \frac{\ln \left(0.15 k_{1} \eta_{\mathrm{eq}}\right)}{\left(0.27 k_{1} \eta_{\mathrm{eq}}\right)^{2}} \frac{\ln \left(0.15 k_{2} \eta_{\mathrm{eq}}\right)}{\left(0.27 k_{2} \eta_{\mathrm{eq}}\right)^{2}} \\
& +A_{1} \ln \left(B_{1} k_{1} \eta_{\mathrm{eq}}\right) \frac{\ln \left(B_{1} k_{2} \eta_{\mathrm{eq}}\right)}{\left(0.13 k \eta_{\mathrm{eq}}\right)^{2}}-\frac{27}{c_{s}^{4}} k^{2} \\
& \times \frac{\mathbf{k}_{1} \cdot \mathbf{k}_{2}}{k_{1}^{2} k_{2}^{2}} \frac{\left[\ln \left(k \eta_{\mathrm{eq}}\right)\right]^{2}}{\left(k \eta_{\mathrm{eq}}\right)^{2}}+\frac{3}{2} \frac{\mathbf{k}_{1} \cdot \mathbf{k}_{2}}{c_{s}^{2} k_{1}^{2}} \\
& \times 2.2\left[\frac{1.2}{2} \frac{\left[\ln \left(k_{1} c_{s} \eta_{\mathrm{eq}}\right)\right]^{2}}{\left(0.13 k \eta_{\mathrm{eq}}\right)^{2}}-\ln \left(k_{1} c_{s} \eta_{\mathrm{eq}}\right)\right. \\
& \left.\left.\times \frac{\ln \left(B_{1} k_{2} \eta_{\mathrm{eq}}\right)}{\left(0.13 \eta_{\mathrm{eq}}\right)^{2}}+(1 \longleftrightarrow 2)\right]\right] \Psi_{\mathbf{k}_{1}}^{(1)}(0) \Psi_{\mathbf{k}_{2}}^{(1)}(0) .
\end{aligned}
$$

In (341), the initial condition $\Psi_{m}^{(2)}(0)$ is given by (349) and $\Psi^{(1)}$ is given by (344). The integration constants can be fixed by comparing at $\eta \simeq \eta_{\mathrm{eq}}$ the oscillating part of (341) to the solution $\Delta_{00}^{(2)}$ obtained for modes crossing the horizon before equality and for $\eta<\eta_{\mathrm{eq}},(256)$. Thus, for $\eta \gg \eta_{\mathrm{eq}}$ and $k \gg$ $\eta_{\text {eq }}^{-1}$, we find that

$$
\begin{aligned}
\Delta_{00}^{(2)}= & -4(1+R) \Psi^{(2)}\left(\eta_{\mathrm{eq}}\right)+\bar{A} \cos \left(k c_{s} \eta\right) \\
& -\frac{2}{7}(1+R) G\left(\mathbf{k}_{1}, \mathbf{k}_{2}, \mathbf{k}\right) \eta^{2} \Psi_{\mathbf{k}_{1}}^{(1)}\left(\eta_{\mathrm{eq}}\right) \Psi_{\mathbf{k}_{2}}^{(1)}\left(\eta_{\mathrm{eq}}\right),
\end{aligned}
$$

where

$$
\begin{aligned}
& \bar{A}= 6 \Psi^{(2)}(0)-\frac{6\left(\mathbf{k} \cdot \mathbf{k}_{1}\right)\left(\mathbf{k} \cdot \mathbf{k}_{2}\right)}{c_{s}^{4} k_{1} k_{2} \cos \left(k c_{s} \eta_{\mathrm{eq}}\right)} \Psi_{\mathbf{k}_{1}}^{(1)}(0) \Psi_{\mathbf{k}_{2}}^{(1)}(0) \\
& \times\left[2 k_{1} k_{2} \cos \left(k_{1} c_{s} \eta_{\mathrm{eq}}\right) \cos \left(k_{2} c_{s} \eta_{\mathrm{eq}}\right)-2 k_{1} k_{2} \cos \left(k c_{s} \eta_{\mathrm{eq}}\right)\right. \\
&\left.\quad+\left(k_{1}^{2}+k_{2}^{2}-k^{2}\right) \sin \left(k_{1} c_{s} \eta_{\mathrm{eq}}\right) \sin \left(k_{2} c_{s} \eta_{\mathrm{eq}}\right)\right] \\
& \times \frac{1}{k_{1}^{4}+k_{2}^{4}+k^{4}-2 k_{1}^{2} k_{2}^{2}-2 k_{1}^{2} k^{2}-2 k_{2}^{2} k^{2}},
\end{aligned}
$$

and $\Psi^{(2)}(0)$ is given in $(272)$.

\section{Secondary Effects and Contamination to Primordial NG}

Given that a detection of a sizable primordial nonGaussianity (and its shape) would represent a real breakthrough into the understanding of the dynamics of the universe during its very first stages, it is crucial that all sources of contamination to the primordial signal are well understood and kept under control. In fact, any nonlinearities can make initially Gaussian perturbations nonGaussian. Such nonprimordial effects can thus complicate the extraction of the primordial non-Gaussianity: we have to be sure we are not ascribing a primordial origin to a signal that is extracted from the CMB (or LSS) data using estimators of non-Gaussianity when that signal has a different origin. Moreover, as stressed in Section 7.1, we must always specify of which primordial non-Gaussianity we study the contamination from the nonprimordial sources (e.g., primordial non-Gaussianity of "local", "equilateral", or "folded" shape).

Broadly speaking, nonprimordial sources of nonGaussianity can be classified into four categories: instrumental systematic effects; residual foregrounds and unresolved point sources; some well-known secondary CMB anisotropies, such as Sunyaev-Zel'dovich (SZ) effect, gravitational lensing, Rees-Sciama effect, and finally previously unknown effects coming from nonlinearities in the Boltzmann equations, which are related to the nonlinear nature of General Relativity and to the nonlinear dynamics of the photon-baryon system. This paper focuses on the last category, but in this section, we offer also a summary of some recent results of the other types of possible contaminations. Before doing that, however, it is important to be more precise about how secondary effects may impact the extraction of a primordial non-Gaussian signal. Mainly, they act in two ways. They might "mimic" a three-point correlation function 
similar in shape to the primordial one. This would produce a bias or a contamination to the estimator of primordial non-Gaussianity. On the other, hand secondary effects might increase the variance of the estimator without contributing to the signal-to-noise ratio. In other words, in this case they degrade the signal-to-noise ratio. Let us define in a quantitative way how to characterize these effects.

8.1. Signal-to-Noise Ratio, Shapes, and Contamination to Primordial Non-Gaussianity. The angular bispectrum, $B_{\ell_{1} \ell_{2} \ell_{3}}$, the harmonic transform of the angular three-point function, of the CMB anisotropies is often used to measure nonGaussianity (see, e.g., [5]). From the usual spherical harmonic coefficients of the temperature anisotropies

$$
a_{\ell m}=\int d^{2} \mathbf{n} Y_{\ell m}^{*}(\mathbf{n}) \frac{\Delta T(\mathbf{n})}{T}
$$

the angle-averaged bispectrum is given by (for more details see, e.g., [43])

$$
B_{\ell_{1} \ell_{2} \ell_{3}} \equiv \sum_{\text {all } m}\left(\begin{array}{ccc}
\ell_{1} & \ell_{2} & \ell_{3} \\
m_{1} & m_{2} & m_{3}
\end{array}\right)\left\langle a_{\ell_{1} m_{1}} a_{\ell_{2} m_{2}} a_{\ell_{3} m_{3}}\right\rangle
$$

where the brackets indicate ensemble average.

We shall quantify the degree to which the primordial and the secondary bispectra are correlated, as well as their expected signal-to-noise ratio following a method that as now become standard $[41,43,76]$. Namely, in the limit of weak non-Gaussianity, one can introduce the Fisher matrix for the amplitudes of the bispectra, $F_{i j}$, given by

$$
F_{i j} \equiv \sum_{2 \leq \ell_{1} \leq \ell_{2} \leq \ell_{3}} \frac{B_{\ell_{1} \ell_{2} \ell_{3}}^{(i)} B_{\ell_{1} \ell_{2} \ell_{3}}^{(j)}}{\sigma_{\ell_{1} \ell_{2} \ell_{3}}^{2}}
$$

where the variance of the bispectrum is

$$
\sigma_{\ell_{1} \ell_{2} \ell_{3}}^{2} \equiv\left\langle B_{\ell_{1} \ell_{2} \ell_{3}}^{2}\right\rangle-\left\langle B_{\ell_{1} \ell_{2} \ell_{3}}\right\rangle^{2} \approx C_{\ell_{1}} C_{\ell_{2}} C_{\ell_{3}} \Delta_{\ell_{1} \ell_{2} \ell_{3}},
$$

and $\Delta_{\ell_{1} \ell_{2} \ell_{3}}$ takes values 1,2 , and 6 when all $\ell^{\prime}$ s are different, two of them are equal and all are the same, respectively. The power spectrum, $C_{\ell}$, is the sum of the theoretical CMB and the detector noise.

The signal-to-noise ratio is given by

$$
\left(\frac{S}{N}\right)_{i}=\frac{1}{\sqrt{F_{i i}^{-1}}}
$$

and we define the cross-correlation coefficient between different shapes $i$ and $j, r_{i j}$, as

$$
r_{i j} \equiv \frac{F_{i j}^{-1}}{\sqrt{F_{i i}^{-1} F_{j j}^{-1}}},
$$

which does not depend on the amplitudes of the different bispectra, but on the shapes, thus measuring how similar are two bispectra $i$ and $j$. One can also define a degradation parameter $d_{i}=F_{i i} F_{i i}^{-1}$ for a degraded $(S / N)$ due to $r_{i j}$.
If a secondary bispectra has some correlation with the primordial one, one expects that the signal-to-noise ratio will be degraded: if one does not account for any secondary bispectra $(S / N)_{0}=\sqrt{F_{\text {prim,prim }}}$ while, marginalizing over a (single) secondary bispectrum, $(S / N)_{\text {prim }}$ gets modified from its zero-order value to $(S / N)_{\text {prim }}=(S / N)_{0}\left(1-r_{i j}^{2}\right)^{1 / 2}$. This means that the minimum detectable value of the primordial nonlinearity parameter $f_{\mathrm{NL}}$ which is obtained by imposing $(S / N)_{\text {prim }}=1$ (or the $1-\sigma$ uncertainty on $f_{\mathrm{NL}}$ given by $\delta f_{\mathrm{NL}}=$ $\left.\sqrt{\left.(F)_{\text {prim,prim }}^{-1}\right|_{f_{\mathrm{NL}}=1}}\right)$ gets shifted by a quantity $\Delta f_{\mathrm{NL}} /\left(f_{\mathrm{NL}}\right)_{0}=$ $\left(1-r_{i j}^{2}\right)^{-1 / 2}-1$.

How much does a given secondary bispectra contaminate the extraction of the primordial bispectrum? If, for example, the predicted shape of the secondary bispectra is sufficiently different from that of the primordial bispectrum, then one would hope that the contamination would be minimal. We can quantify the contamination of the primordial bispectrum as follows: we fit the primordial bispectrum template to the second-order bispectrum, and find the bestfitting $f_{\mathrm{NL}}^{\text {con }}$ ("con" stands for contamination) by minimizing the $\chi^{2}$ given by

$$
\chi^{2}=\sum_{2 \leq \ell_{1} \leq \ell_{2} \leq \ell_{3}} \frac{\left(f_{\mathrm{NL}} B_{\ell_{1} \ell_{2} \ell_{3}}^{\text {prim }}-B_{\ell_{1} \ell_{2} \ell_{3}}^{2 \text { nd }}\right)^{2}}{\sigma_{\ell_{1} \ell_{2} \ell_{3}}^{2}},
$$

with respect to $f_{\mathrm{NL}}$. Here, $B_{\ell_{1} \ell_{2} \ell_{3}}^{\text {prim }}$ is the primordial bispectrum with amplitude $f_{\mathrm{NL}}=1[43]$. We obtain

$$
\begin{aligned}
f_{\mathrm{NL}}^{\mathrm{con}} & =\frac{1}{N} \sum_{2 \leq \ell_{1} \leq \ell_{2} \leq \ell_{3}} \frac{B_{\ell_{1} \ell_{2} \ell_{3}}^{2 \mathrm{nd}} \mathrm{B}_{\ell_{1} \ell_{2} \ell_{3}}^{\mathrm{prim}}}{\sigma_{\ell_{1} \ell_{2} \ell_{3}}^{2}}, \\
N & =\sum_{2 \leq \ell_{1} \leq \ell_{2} \leq \ell_{3}} \frac{\left(B_{\ell_{1} \ell_{2} \ell_{3}}^{\text {prim }}\right)^{2}}{\sigma_{\ell_{1} \ell_{2} \ell_{3}}^{2}} .
\end{aligned}
$$

This is the value of $f_{\mathrm{NL}}$ one would find, if one did not know that the primordial bispectrum did not exist but there was only the secondary bispectrum. In other words, the effective nonlinearity parameter $f_{\mathrm{NL}}^{\mathrm{con}}$ gives the value that a bispectrum estimator designed for constraining primordial non-Gaussianity would measure due to the presence of the secondary bispectrum $B_{\ell_{1} \ell_{2} \ell_{3}}^{2 \text { nd }}$. As one can see $f_{\mathrm{NL}}^{\text {con }}$ turns out to be proportional to the mixed entry of the Fisher matrix, since this governs the correlation between different types of bispectra on the basis of their shape dependence.

8.2. Some "well-known" Secondary Sources of NonGaussianity. Instrumental systematic effects are one of the principal issues in the search for primordial non-Gaussianity. Since they depend on the specific instrument, here we do not intend to go into any detail, but we refer the reader to [77] as an interesting example of this kind of effects for the currently flying mission of the satellite Planck. Residual foregrounds and unresolved point sources represent other important possible contaminants and particular attention have been devoted to them in the analysis of the WMAP data $[44,57,72]$. For an experiment like WMAP secondary 
effects such as Sunyaev-Zel'dovich effect, gravitational lensing, Integrated-Sachs-Wolfe (or Rees-Sciama) effect should be not so relevant for $\ell<500$ with a bias of just 1.5 to 2 [78] for local primordial non-Gaussianity (see also $[43,44]$ ). (In order to understand the relative importance of the bias from a given secondary effect let us recall that, including the information from polarization, the minimum detectable (local) $f_{\mathrm{NL}}^{\text {loc }}$ for an experiment like WAMP is of the order of 10 while for Planck it is of the order of 3; the minimum detectable value for an equilateral primordial non-Gaussianity is $f_{\mathrm{NL}}^{\mathrm{eq}} \simeq 30$ for Planck, see, for example, [4, 43, 71, 79].) However, for higher resolution and more sensitive experiments like Planck, such effects must be taken into account with care. Here, we report the results of some recent analyses about the contamination to primordial non-Gaussianity from some secondary effects. Such a brief summary is by no means exhaustive. An interesting case is given by the cross-correlation of the ISW and the lensing effects, see [41]. In the detailed analysis of [80], it is shown that the ISW-lensing bispectrum produces a bias of $f_{\mathrm{NL}}^{\text {cont }} \simeq 9$ to local primordial non-Gaussianity (see also [78]), while the bias to equilateral primordial non-Gaussianity is negligible (see also $[44,78]$ ). The impact is mainly on the local type of primordial non-Gaussianity because the ISW-lensing correlation correlates the large-scale gravitational potential fluctuations sourcing the ISW effect with the small scale lensing effects of the $\mathrm{CMB}$, thus producing a bispectrum which peaks in the squeezed configurations as the local shape. As far as the increase of the variance of the estimator of primordial non-Gaussianity induced by $\mathrm{CMB}$ lensing is concerned it turns out to be of the order of $20 \%$ for an experiment like Planck (see [79, 80]). Notice that interestingly enough, gravitational lensing can also have another peculiar effect on the extraction of primordial non-Gaussianity: it can modify its shape by smoothing its acoustic features. However, according to the analysis of [80] (and contrary to the results of [81]) such a "distorsion" of the shape should be small, of the order of $10 \%$ for $l<2000$.

A similar analysis has been performed looking also at scales where nonlinear effects can become relevant. In this case a bispectrum from the correlation of the Rees-Sciama and lensing effects has been studied in [82], and it has been found that in this case the bias to a local primordial nonGaussianity corresponds to $f_{\mathrm{NL}}^{\text {cont }} \simeq 10$, agreeing with the results of [80] where the two analyses can be compared.

Another secondary source of contamination that has been studied quite recently in [83] deals with bispectra generated by correlations of number density and lensing magnification of radio and SZ point sources with the ISW effect. Also, in this case the large-scale modulation of small-scale number density due to fluctuations which source the ISW generates a bispectrum which peaks on squeezed configurations and it has been found that it corresponds to a contamination of local primordial nonGaussianity of $f_{\mathrm{NL}}^{\text {cont }} \simeq 1.5$, which should be not so relevant for an experiment like Planck. The novelty of this effect is that it is different from the usual bispectrum of point sources when treated with a Poisson distribution (see, e.g., [43]).
Other secondary effects include, for example, the SZSZ-SZ bispectrum [84] and non-Gaussianities from the kinetic SZ and Ostriker-Vishniac effect [76, 85]. However, the SZ-SZ-SZ bispectrum can be considered as an extra Poisson contribution to the unresolved point sources for $l<1500$ [78]. Moreover, recently an analysis of the bispectrum generated from inhomogeneous reionization has been performed in [86], where it has been shown that it can give rise to a very small contamination to the local primordial non-Gaussianity of $f_{\mathrm{NL}}^{\text {cont }} \simeq-0.1$.

Finally let us also recall some recent studies of a specific effect arising at second-order in perturbation theory, when looking at the fluctuations in the Boltzmann equations for the photon-baryon fluid. In [63, 87-89], the perturbations to the phase of recombination between electrons and baryons has been considered ("inhomogeneous recombination"). It turns out that the electron density perturbation can be of the order of 5 times bigger than the one in the density of the baryons. This gives rise to the prospect of a non-Gaussianity which corresponds to $\left|f_{\mathrm{NL}}^{\text {cont }}\right| \simeq 5$. In fact, the detailed study of this effect shows that the contamination is much smaller. The bispectrum from the perturbed recombination peaks in the squeezed configuration with a contamination to the primordial non-Gaussianity of the local type of $\left|f_{\mathrm{NL}}^{\text {cont }}\right| \simeq 0.7$.

Let us conclude this section by mentioning that the nonlinearities emerging from secondary effects can be very interesting by themselves, since they carry a lot of information about the evolution of the universe and the growth of structures after recombination till very low redshift. Detailed examples and reviews about this aspect can be found, for example, in [90-92].

\section{How to Analytically Estimate the Signal-to-Noise Ratio from the NG at Recombination and Its Contamination to the Primordial NG}

As we have seen in Section 7, the dynamics at recombination is quite involved because all the nonlinearities in the evolution of the baryon-photon fluid at recombination and the ones coming from general relativity should be accounted for. Such a contribution is so relevant because it represents a major part of the second-order radiation transfer function which must be determined in order to have a complete control of both the primordial and nonprimordial part of $\mathrm{NG}$ in the CMB anisotropies. The NG generated at the surface of last scattering comprises various effects. In this section, we devote our attention to one particular relevant contribution, the one coming from the nonlinear evolution of the second-order gravitational potential which grows in time on small scales. The analysis of this contribution offers the opportunity to understand how some secondary effects can contaminate mostly a given type of primordial NG, while leaving almost untouched other different forms of primordial NG. In this case, this effect is a causal one, developing on small scales, so we expect that the NG it generates will be mainly of the equilateral type, rather than of local type. Therefore, a reasonable question is to 
which extent the NG from recombination alters the possible detection of the primordial NG of the equilateral type. The goal of this Section is therefore to illustrate how to estimate in a semianalytical way the contribution to NG from recombination.

9.1. Signal-to-Noise Ratio for the Primordial Equilateral Bispectrum. In this subsection we wish to recover the estimate for the signal-to-noise ratio $(S / N)$ given in [44] for the primordial bispectra of "equilateral" type [93] by adopting a simple model. In other words, we test the goodness of the semi-analytical model we will be using in the next Section to estimate the bispectrum from the recombination era.

Our starting point is the primordial equilateral bispectrum [93]

$$
\begin{aligned}
& \left\langle\Phi\left(\mathbf{k}_{1}\right) \Phi\left(\mathbf{k}_{2}\right) \Phi\left(\mathbf{k}_{3}\right)\right\rangle \\
& =(2 \pi)^{3} \delta^{(3)}\left(\mathbf{k}_{1}+\mathbf{k}_{2}+\mathbf{k}_{3}\right) B_{\text {equil }}\left(k_{1}, k_{2}, k_{3}\right),
\end{aligned}
$$

where

$$
\begin{aligned}
B_{\text {equil }}\left(k_{1}, k_{2}, k_{3}\right) & \\
=f_{\mathrm{NL}}^{\text {equil }} \cdot 6 A^{2} \cdot( & -\frac{1}{k_{1}^{3} k_{2}^{3}}-\frac{1}{k_{1}^{3} k_{3}^{3}}-\frac{1}{k_{2}^{3} k_{3}^{3}} \\
& \left.-\frac{2}{k_{1}^{2} k_{2}^{2} k_{3}^{2}}+\frac{1}{k_{1} k_{2}^{2} k_{3}^{3}}+(5 \text { perm. })\right),
\end{aligned}
$$

and the permutations act only on the last term in parentheses. The parameter $f_{\mathrm{NL}}^{\text {equil }}$ quantifies the level of NG while $A=$ $17.46 \times 10^{-9}$ is the amplitude of the primordial gravitional potential power spectrum computed at first order

$$
\left\langle\Phi^{(1)}\left(\mathbf{k}_{1}\right) \Phi^{(1)}\left(\mathbf{k}_{2}\right)\right\rangle=(2 \pi)^{3} \delta^{(3)}\left(\mathbf{k}_{1}+\mathbf{k}_{2}\right) P\left(k_{1}\right),
$$

with $P(k)=A / k^{3}$. Since the signal-to-noise ratio $(S / N)$ will be some function of the maximum multipole a given experiment can reach, $\ell_{\max } \gg 1$, we can use the flat-sky approximation $[79,94]$ and write for the bispectrum

$$
\left\langle a\left(\vec{\ell}_{1}\right) a\left(\vec{\ell}_{2}\right) a\left(\vec{\ell}_{3}\right)\right\rangle=(2 \pi)^{2} \delta^{(2)}\left(\vec{\ell}_{123}\right) B\left(\ell_{1}, \ell_{2}, \ell_{3}\right),
$$

where $\vec{\ell}_{123}=\vec{\ell}_{1}+\vec{\ell}_{2}+\vec{\ell}_{3}$, with [25]

$$
\begin{aligned}
& B_{\text {equil }}\left(\ell_{1}, \ell_{2}, \ell_{3}\right) \\
& =\frac{\left(\eta_{0}-\eta_{r}\right)^{2}}{(2 \pi)^{2}} \int d k_{1}^{z} d k_{2}^{z} d k_{3}^{z} \delta^{(1)} \\
& \quad \times\left(k_{123}^{z}\right) B_{\text {equil }}\left(k_{1}^{\prime}, k_{2}^{\prime}, k_{3}^{\prime}\right) \tilde{\Delta}^{T}\left(\ell_{1}, k_{1}^{z}\right) \tilde{\Delta}^{T}\left(\ell_{2}, k_{2}^{z}\right) \tilde{\Delta}^{T}\left(\ell_{3}, k_{3}^{z}\right),
\end{aligned}
$$

where $k^{\prime}$ means $k$ evaluated such that $\overrightarrow{k^{\|}}=\vec{\ell} /\left(\eta_{0}-\eta_{r}\right)$ and

$$
\begin{aligned}
\tilde{\Delta}^{T}\left(\ell, k^{z}\right)= & \int_{0}^{\eta_{0}} \frac{d \eta}{\left(\eta_{0}-\eta\right)^{2}} \\
& \times S\left(\sqrt{\left(k^{z}\right)^{2}+\ell^{2} /\left(\eta_{0}-\eta\right)^{2}}, \eta\right) e^{i k^{z}\left(\eta_{r}-\eta\right)}
\end{aligned}
$$

is the radiation transfer function defined by the CMB source function $S(k, \eta)$. In this notation, $\eta_{0}$ and $\eta_{r}$ represent the present-day and the recombination conformal time, respectively and $k^{z}$ and $\vec{k} \|$ are the momentum components perpendicular and parallel, respectively, to the plane orthogonal to the line-of-sight.

The $(S / N)$ ratio in the flat-sky formalism is $[79,94]$

$$
\begin{aligned}
\left(\frac{S}{N}\right)^{2}= & \frac{f_{\text {sky }}}{\pi} \frac{1}{(2 \pi)^{2}} \int d^{2} \ell_{1} d^{2} \ell_{2} d^{2} \ell_{3} \delta^{(2)} \\
& \times\left(\vec{\ell}_{123}\right) \frac{B_{\text {equil }}^{2}\left(\ell_{1}, \ell_{2}, \ell_{3}\right)}{6 C\left(\ell_{1}\right) C\left(\ell_{2}\right) C\left(\ell_{3}\right)},
\end{aligned}
$$

where $f_{\text {sky }}$ stands for the portion of the observed sky. In order to compute the bispectrum $B_{\text {equil }}\left(\ell_{1}, \ell_{2}, \ell_{3}\right)$ and the power spectrum $C(\ell)$, we adopt the following model

$$
a(\vec{\ell})=\int \frac{d k^{z}}{2 \pi} e^{i k^{z}\left(\eta_{0}-\eta_{r}\right)} \Phi\left(\mathbf{k}^{\prime}\right) \tilde{\Delta}^{T}\left(\ell, k^{z}\right)
$$

where we mimic the effects of the transfer function on small scales as

$$
\tilde{\Delta}^{T}\left(\ell, k^{z}\right)=a\left(\eta_{0}-\eta_{r}\right)^{-2} e^{-1 / 2\left(\ell / \ell_{*}\right)^{1.2}} e^{-1 / 2\left(\left|k_{z}\right| / k_{*}\right)^{1.2}}
$$

That is, a simple exponential and a normalization coefficent $a$ to be determined to match the amplitude of the angular power spectrum at the characteristic scale $\ell \simeq \ell_{*}=$ $k_{*}\left(\eta_{0}-\eta_{r}\right)$. (We could equally choose a transfer function as $\tilde{\Delta}^{T}\left(\ell, k^{z}\right)=a\left(\eta_{0}-\eta_{r}\right)^{-2} e^{-1 / 2\left(\ell / \ell_{*}\right)^{1.2}} \theta\left(k_{*}-\left|k^{z}\right|\right)$, the relevant approximation being that the integral over $k^{z}$ is cut at the scale $k_{*}$.) It is important to make clear what are the reasons underlying the choice of such a model. When computing the $(S / N),(367)$ with $\ell_{*}=k_{*}\left(\eta_{0}-\eta_{r}\right) \simeq 750$ and $a \simeq 3$ is able to account for the combined effects of "radiation driving", which occurs at $\ell>\ell_{\mathrm{eq}} \simeq 160$ and boosts the angular power spectrum with respect to the Sachs-Wolfe plateau, and the effects of Silk damping which tend to suppress the CMB anisotropies for scales $\ell>\ell_{D} \simeq 1300$. The combination of these effects produces a decrease in the angular power spectrum from a scale $\ell_{*} \simeq 750$. (The choice of the exponent 1.2 derives from the study of the diffusion damping envelope in [95]). The power spectrum in the flat-sky approximation is given by $\left\langle a\left(\vec{l}_{1}\right) a\left(\vec{l}_{2}\right)\right\rangle=(2 \pi)^{2} \delta^{(2)}\left(\vec{l}_{12}\right) C\left(\ell_{1}\right)$ with

$$
C(\ell)=\frac{\left(\eta_{0}-\eta_{r}\right)^{2}}{(2 \pi)} \int d k^{z}\left|\tilde{\Delta}^{T}\left(\ell, k^{z}\right)\right|^{2} P(k) .
$$


The exponential of the transfer function for (367) allows to cut off the integral for $k \simeq k_{*}$ and one finds (see also [79])

$$
C(\ell)=a^{2} \frac{A}{\pi \ell^{2}} \frac{e^{-\left(\ell / \ell_{*}\right)^{1.2}}}{\sqrt{1+\ell^{2} / \ell_{*}^{2}}} \simeq a^{2} \frac{A}{\pi} \frac{\ell_{*}}{\ell^{3}} e^{-\left(\ell / \ell_{*}\right)^{1.2}}
$$

where the last equality holds for $\ell \gg \ell_{*}$. To compute the bispectrum we proceed in a similar way. One first uses the Dirac deltas, $\delta^{(1)}\left(k_{123}^{z}\right)$ and $\delta^{(2)}\left(\vec{\ell}_{123}\right)$. Then, it proves to be useful the change of variable $k_{1}^{z}=x_{1} \ell_{1} /\left(\eta_{0}-\eta_{r}\right), k_{2}^{z}=$ $x_{2} \ell_{2} /\left(\eta_{0}-\eta_{r}\right)$. In this way, the transfer functions become $\widetilde{\Delta}^{T}\left(\ell_{i}, k_{i}^{z}\right) \propto e^{-1 / 2\left(\left|x_{i}\right| \ell_{i} / \ell_{*}\right)^{1.2}}$ which allows to cut the integrals over $x_{i}(i=1,2)$ at $\ell_{*} / \ell_{i}$. Now, as a good approximation to see the effects of the transfer functions, we can take $\ell \gg \ell_{*}$ and thus the integral over $x_{i}$ can be easily computed by just evaluating the integrand in $x_{i}=0$ times $4\left(\ell_{*} / \ell_{1}\right)\left(\ell_{*} / \ell_{2}\right)$. With this approximation the integral in $k_{i}^{z}$ is easily obtained and we get for the bispectrum

$$
\begin{aligned}
& B_{\text {equil }}\left(\ell_{1}, \ell_{2}, \ell_{3}\right) \\
& =\frac{24 f_{1}}{(2 \pi)^{2}} f_{\mathrm{NL}}^{\mathrm{equil}} a^{3} A^{2} e^{-\left(\ell_{1}^{1.2}+\ell_{2}^{1.2}+\ell_{3}^{1.2}\right) / 2 \ell_{*}^{1.2}} \ell_{*}^{2} \\
& \quad \times\left(-\frac{1}{\ell_{1}^{3} \ell_{2}^{3}}-\frac{1}{\ell_{1}^{3} \ell_{3}^{3}}-\frac{1}{\ell_{2}^{3} \ell_{3}^{3}}-\frac{2}{\ell_{1}^{2} \ell_{2}^{2} \ell_{3}^{2}}\right. \\
& \left.\quad+\frac{1}{\ell_{1} \ell_{2}^{2} \ell_{3}^{3}}+(5 \text { perm. })\right),
\end{aligned}
$$

where

$$
\ell_{3}^{2}=\ell_{1}^{2}+\ell_{2}^{2}+2 \overrightarrow{\ell_{1}} \cdot \vec{\ell}_{2}
$$

The coefficient $f_{1} \simeq 1 / 1.4=0.7$ is a fudge factor that improves the matching between our approximation for the bispectrum and numerical results that have been consistenly checked. Notice that according to our approximation, the equilateral structure of (361) is preserved in $\ell$ space. The expression (371) can be also written as $B_{\text {equil }}\left(\ell_{1}, \ell_{2}, \ell_{3}\right)$ $=(2 \pi)^{-2} 48 f_{1} f_{\mathrm{NL}}^{\text {equil }} a^{3} A^{2} \ell_{*}^{2} e^{-\left(\ell_{1}^{1.2}+\ell_{2}^{1.2}+\ell_{3}^{1.2}\right) / 2 \ell_{*}^{1.2}}(1+\cos \theta)\left(\ell_{1}+\right.$ $\left.\ell_{2}-\ell_{3}\right) / \ell_{1}^{2} \ell_{2}^{2} \ell_{3}^{3}, \theta$ being the angle between $\vec{\ell}_{1}$ and $\vec{\ell}_{2}$. In computing the signal-to-noise ratio, consistency with our approximation (371) requires that we integrate over $\ell_{1}, \ell_{2}$ starting from a minimum $\ell_{\min }>\ell_{*}$ up to $\ell_{\max }$ and paying attention to the fact that even $\ell_{3}$ in (372) must be larger than $\ell_{\min }$. The scaling with $\ell_{\max }$ with respect to the case of a local type bispectrum turns out to be much milder. While for the local type $(S / N)^{2} \propto \ell_{\max }^{2}$ [79], for the equilateral bispectrum (360), we find $(S / N)^{2} \propto \ell_{\max }$ and, setting $\ell_{*}=750$ and $\ell_{\min } \simeq 1200$,

$$
\begin{aligned}
\left(\frac{S}{N}\right)_{\text {equil }}^{2} & =0.48 \times 10^{5} \frac{f_{\text {sky }}}{2^{5} \pi^{3} 6} A\left(f_{\mathrm{NL}}^{\text {equil }}\right)^{2} \ell_{\max } \\
& \simeq 8 f_{\text {sky }} A\left(f_{\mathrm{NL}}^{\text {equil }}\right)^{2} \ell_{\max .}
\end{aligned}
$$

(these scalings can be easily understood by analyzing the expressions $(S / N)^{2}$ for the local and equilateral primordial NG. In the local case, $(S / N)^{2}$ is proportional to $\int d^{2} \ell_{1} d^{2} \ell_{2} d^{2} \ell_{3} \delta^{(2)}\left(\vec{\ell}_{123}\right)\left(\ell_{1}^{3}+\ell_{2}^{3}+\ell_{3}^{3}\right)^{2} /\left(\ell_{1} \ell_{2} \ell_{3}\right)^{3}$ [79]; since the squeezed configuration, e.g., $\ell_{1} \ll \ell_{2}, \ell_{3}$, is dominating the local bispecrum, the integral becomes proportional to $\int d \ell_{1} d \ell_{2}\left(\ell_{2} / \ell_{1}^{2}\right) \propto \ell_{\max }^{2}$. In the equilateral case, however, $(S / N)^{2}$ receives contributions from the configuration which is peaked at $\ell_{1} \sim \ell_{2} \sim \ell_{3}$ and therefore it can be written as $\left.\int d^{2} \ell_{1} d^{2} \ell_{2} \delta\left(\ell_{1}-\ell_{2}\right) / \ell_{1}^{2} \propto \ell_{\max }\right)$. By choosing $f_{\text {sky }}=0.8$ and $\ell_{\max }=2000$ we find a minimum detectable

$$
f_{\mathrm{NL}}^{\text {equil }} \simeq 66,
$$

obtained imposing $(S / N)_{\text {equil }}=1$. Both the estimate of the minimum value of $f_{\mathrm{NL}}^{\text {equil }}$ and the scaling $(S / N)^{2} \propto \ell_{\max }$ are in remarkable agreement with the result obtained in [44] where the full transfer function is used and a value of $f_{\mathrm{NL}}^{\text {equil }}=67$ is obtained. (We thank M. Liguori for discussions about the minimum value of $f_{\mathrm{NL}}^{\text {equil }}$ detectable by Planck and for its scaling with $\ell_{\max }$.) Notice that our estimate is independent from the coefficient $a$ and the exponential $e^{-1 / 2\left(\ell / \ell_{*}\right)^{1.2}}$ introduced below (367) to mimic the full transfer function. This is because there is an equal number of transfer functions in the numerator and denominator of the expression (366) for the signal-to-noise ratio and their effect tend to cancel despite they are not simple multiplicative factors (see discussion in [79]).

9.2. Non-Gaussianity from Recombination. Comforted by the goodness of our model, in this section, we wish to estimate the level of NG generated at the recombination era. One can check that on small scales the second-order anisotropies are dominated by the second-order gravitational potential $\Phi^{(2)}$ which grows as $\eta^{2}$, as first pointed out in [48]. Let us first briefly show how this result can be obtained using the calculations of Section 7. Consider the tight coupling regime, and small scales, well inside the diffusion Silk-damping scale $\lambda_{D}$. Then, look at (232). One realizes that, apart from the combination $\Delta_{00}^{(2)}+4 \Phi^{(2)}$, all the other terms scale at most as $\eta$ or they are suppressed either because in the tight coupling regime or because of the damping diffusion on small scales. On the other hand, one finds that the main contribution to the bispectrum generated at recombination comes from

$$
\Theta_{\mathrm{SW}}^{(2)}=\frac{1}{4} \Delta_{00}^{(2)}+\Phi^{(2)}
$$

which is the usual term appearing in the $\mathrm{CMB}$ anisotropies due to the intrinsic photon energy density fluctuations $\Delta_{00}^{(2)}$ and the gravitational redshift due to the potential. In fact, 
such a term on large scales reduces to the Sachs-Wolfe effect, while on small scales at recombination, using (282) and (285), we find

$$
\begin{aligned}
\frac{1}{4} \Delta_{00}^{(2)}+\Phi^{(2)} \simeq & -R \Phi^{(2)} \\
= & -\frac{R \eta_{r}^{2}}{14} G\left(\mathbf{k}_{1}, \mathbf{k}_{2}, \mathbf{k}\right) T\left(k_{1}\right) \Phi^{(1)} \\
& \times\left(\mathbf{k}_{1}\right) T\left(k_{2}\right) \Phi^{(1)}\left(\mathbf{k}_{2}\right),
\end{aligned}
$$

where we have evaluated the expression at the recombination time $\eta_{r}$ and $R=3 \rho_{\mathrm{b}} / 4 \rho_{\gamma}$ is the baryon-to-photon energy density ratio. In writing (376), we have dropped off the contribution from primordial non-Gaussianity (which, anyway, always propagates linearly) since here we are focusing on the relevant secondary effects that can constitute a source of contamination to it (see (202) and the discussion below). Notice also that this expression has been obtained assuming all the momenta much larger than $k_{\text {eq }}$.

Equation (376) has been confirmed numerically in [48]. There is also another way to obtain the analytical expression in the first line of (376), as discussed in details in [48], which exploits some well-known results at linear order extending them at second-order. At second order, in the perturbations the solution for the acoustic oscillations will have a form very similar to the linear solution written in (C.18), except, as usual, of some source terms $S$ made by products of first-order perturbations.

$$
\frac{\Delta_{00}^{(2)}}{4}=\left[\frac{\Delta_{00}^{(2)}}{4}(0)+(1+R) \Phi^{(2)}\right] \cos \left(k r_{s}\right)-(1+R) \Phi^{(2)}+S
$$

Now on sufficiently small scales the products of first-order terms are indeed suppressed, due either to Silk damping, or because gravitational potentials are suppressed as $\left(k / k_{\mathrm{eq}}\right)^{2}$. Recall that only acoustic oscillations are damped by Silk damping diffusion, so that the cosine in (377) is multiplied by $e^{-\left(k / k_{D}\right)^{2}} k \gg k_{D}$, where $k_{D}^{-1}=\lambda_{D}$ indicates damping length, but the baryon induced shift $(1+R) \Phi^{(1)}$ is left untouched. Therefore, on small scales the combination of the damping effects and the growth of the second-order gravitational potential $\Phi^{(2)}$ as $\eta^{2}$ single out the dominant effect in (377) to be $\Theta_{\mathrm{SW}}^{(2)} \simeq-R \Phi^{(2)}$.

9.2.1. Bispectrum from Recombination due to the Nonlinear Growth of the Gravitational Potential. Let us start to go into some details of the form of the bispectrum induced by the contribution (376). In (376), the kernel $G$ is given by

$$
G\left(\mathbf{k}_{1}, \mathbf{k}_{2}, \mathbf{k}\right)=\mathbf{k}_{1} \cdot \mathbf{k}_{2}-\frac{10}{3} \frac{\left(\mathbf{k} \cdot \mathbf{k}_{1}\right)\left(\mathbf{k} \cdot \mathbf{k}_{2}\right)}{k^{2}}
$$

From the form of the kernel we see that the NG at recombination is dominated by an equilateral configuration, as expected from the fact that its origin is gravitational. Here, and in the following, we are implicitly assuming that a convolution is acting on the kernel as

$$
\begin{aligned}
& \frac{1}{(2 \pi)^{3}} \int d \mathbf{k}_{1} d \mathbf{k}_{2} \delta^{(3)}\left(\mathbf{k}_{1}+\mathbf{k}_{2}+\mathbf{k}_{3}\right) \\
& \quad \times G\left(\mathbf{k}_{1}, \mathbf{k}_{2}, \mathbf{k}\right) T\left(k_{1}\right) \Phi^{(1)}\left(\mathbf{k}_{1}\right) T\left(k_{2}\right) \Phi^{(1)}\left(\mathbf{k}_{2}\right) .
\end{aligned}
$$

The reader should remember that, at first order in perturbation theory, the combination $\Theta_{\mathrm{SW}}^{(1)}+R \Phi^{(1)}$ is exponentially suppressed by the Silk damping, but still greater than the term $R \Phi^{(1)}$ (which does not suffer the damping) for the maximum multipole of interest, $\ell_{\max } \sim 2000$. This is mainly due to the fact that the first-order gravitational potential rapidly decays on small scales. On the contrary, at second-order in perturbation theory, the gravitational potential grows like the scale factor on small scales and it turns out that the $R \Phi^{(2)}$ dominates on small scales (see [48]).

The gravitational potential at linear order can be expressed as usual in terms of the transfer function $T(k)$

$$
T(k) \approx 12\left(\frac{k_{\mathrm{eq}}}{k}\right)^{2} \ln \left[k / 8 k_{\mathrm{eq}}\right]
$$

where the last step is an approximation valid on scales smaller than the equivalence scale, $k \gg k_{\text {eq }}$. In the following, we will account for the logarithmic growth just with a coeffcient $T_{0}(k)=12 \ln \left[k / 8 k_{\text {eq }}\right] \approx 11$ for the scales of interest.

In the flat-sky approximation, one arrives at an expression similar to (364), where now one of the linear transfer functions must replaced by a transfer function at secondorder. Specifically, one finds

$$
\begin{aligned}
& B_{\text {rec }}\left(\ell_{1}, \ell_{2}, \ell_{3}\right) \\
& =\frac{\left(\eta_{0}-\eta_{r}\right)^{2}}{(2 \pi)^{2}} \int d k_{1}^{z} d k_{2}^{z} d k_{3}^{z} \delta^{(1)}\left(k_{123}^{z}\right) \\
& \times\left[G\left(\mathbf{k}_{1}^{\prime}, \mathbf{k}_{2}^{\prime}, \mathbf{k}_{3}^{\prime}\right) T\left(k_{1}^{\prime}\right) T\left(k_{2}^{\prime}\right) P\left(k_{1}^{\prime}\right) P\left(k_{2}^{\prime}\right)\right. \\
& \left.\quad \times \tilde{\Delta}^{T}\left(\ell_{1}, k_{1}^{z}\right) \tilde{\Delta}^{T}\left(\ell_{2}, k_{2}^{z}\right) \tilde{\Delta}^{T(2)}\left(\ell_{3}, k_{3}^{z}\right)+\text { cyclic }\right] .
\end{aligned}
$$

By using our model (367) and

$$
\tilde{\Delta}^{T(2)}\left(\ell, k^{z}\right)=-\frac{R}{14} \frac{\tau_{r}^{2}}{\left(\eta_{0}-\eta_{r}\right)^{2}},
$$


for the second-order radiation transfer function, we find

$$
\begin{aligned}
& B_{\text {rec }}\left(\ell_{1}, \ell_{2}, \ell_{3}\right) \\
& =-\frac{R}{14} \frac{\left(\eta_{0}-\eta_{r}\right)^{-4}}{(2 \pi)^{2}} k_{\mathrm{eq}}^{4} \eta_{r}^{2} A^{2} a^{2} T_{0}^{2} e^{-1 / 2\left(\ell_{1} / \ell_{*}\right)^{1.2}} e^{-1 / 2\left(\ell_{2} / \ell_{*}\right)^{1.2}} \\
& \times \int d k_{1}^{z} d k_{2}^{z} d k_{3}^{z} \delta^{(1)}\left(k_{123}^{z}\right) \\
& \times\left[G\left(\mathbf{k}_{1}^{\prime}, \mathbf{k}_{2}^{\prime}, \mathbf{k}_{3}^{\prime}\right) \frac{1}{k_{1}^{\prime 5} k_{2}^{\prime 5}} e^{-1 / 2\left(\left|k_{1 z}\right| / k_{*}\right)^{1.2}}\right. \\
& \left.\quad \times e^{-1 / 2\left(\left|k_{2 z}\right| / k_{*}\right)^{1.2}}+\text { cyclic }\right] .
\end{aligned}
$$

At this point, we proceed further by employing the same approximation described after (370). We use the Dirac delta to replace the variable $k_{3 z}$, and the exponential allow us to evaluate the integral for $k_{1 z}=k_{2 z}=0$, for scales $\ell_{i} \gg \ell_{*}$. This leads to

$$
\begin{aligned}
& B_{\mathrm{rec}}\left(\ell_{1}, \ell_{2}, \ell_{3}\right) \\
& =-\frac{4 f_{2}}{(2 \pi)^{2}} \frac{R}{14} A^{2} a^{2} T_{0}^{2}\left(k_{\mathrm{eq}} \eta_{r}\right)^{2} \ell_{\mathrm{eq}}^{2} \ell_{*}^{2} e^{-1 / 2\left(\ell_{1} / \ell_{*}\right)^{1.2}} e^{-1 / 2\left(\ell_{2} / \ell_{*}\right)^{1.2}} \\
& \quad \times \frac{1}{\ell_{1}^{5} \ell_{2}^{5}}\left[\overrightarrow{\ell_{1}} \cdot \overrightarrow{\ell_{2}}-\frac{10}{3} \frac{\left(\overrightarrow{\ell_{3}} \cdot \overrightarrow{\ell_{1}}\right)\left(\overrightarrow{\ell_{3}} \cdot \overrightarrow{\ell_{2}}\right)}{\ell_{3}^{2}}\right]+\text { cyclic. }
\end{aligned}
$$

Again, here $f_{2}$ is a coefficient to better calibrate our approximations with numerical results that we have performed in order to test the validity of our approach. Not surprisingly, it turns out that $f_{2} \simeq f_{1} \simeq 1 / 1$.

\subsection{Contamination to Primordial Non-Gaussianity from Recombination: Fisher Matrices}

9.3.1. Contamination to Primordial Non-Gaussianity of the Equilateral Type. Our goal now is to quantify the level of NG coming from the recombination era and to estimate the level of degradation it causes on the possible measurement of the equilateral primordial bispectrum. The reader should keep in mind that, given the form of the kernel function (378), the NG from recombination is expected to be of the equilateral type. Following Section 8.1, a rigorous procedure is to define the Fisher matrix (in flat-sky approximation) as

$$
F_{i j}=\int d^{2} \ell_{1} d^{2} \ell_{2} d^{2} \ell_{3} \delta^{(2)}\left(\vec{\ell}_{123}\right) \frac{B^{i}\left(\ell_{1}, \ell_{2}, \ell_{3}\right) B^{j}\left(\ell_{1}, \ell_{2}, \ell_{3}\right)}{6 C\left(\ell_{1}\right) C\left(\ell_{2}\right) C\left(\ell_{3}\right)},
$$

where $i$ (or $j)=$ (rec, equil), and to define the signal-tonoise ratio for a component $i,(S / N)_{i}=1 / \sqrt{F_{i i}^{-1}}$, and the degradation parameter $d_{i}=F_{i i} F_{i i}^{-1}$ due to the correlation between the different components $r_{i j}=F_{i j}^{-1} / \sqrt{F_{i i}^{-1} F_{j j}^{-1}}$. The first entry $F_{\text {equil,equil }}$ of the Fisher matrix corresponds to the
$(S / N)^{2}$ ratio computed in (373) which does not account for any kind of cross-correlation. Due to the equilateral form of the NG generated at recombination, we expect that the minimum value detectable for $f_{\mathrm{NL}}^{\text {equil }}$ will be higher than the one reported in (374). For the mixed entry, we find

$$
\begin{aligned}
F_{\text {rec }, \text { equil }}= & \frac{f_{\text {sky }}}{\pi} \frac{1}{(2 \pi)^{2}} \int d^{2} \ell_{1} d^{2} \ell_{2} d^{2} \ell_{3} \delta^{(2)}\left(\vec{\ell}_{123}\right) \\
& \times \frac{B_{\text {rec }}\left(\ell_{1}, \ell_{2}, \ell_{3}\right) B_{\text {equil }}\left(\ell_{1}, \ell_{2}, \ell_{3}\right)}{6 C\left(\ell_{1}\right) C\left(\ell_{2}\right) C\left(\ell_{3}\right)} \\
= & -3 f_{1} f_{2} \frac{f_{\text {sky }}}{\pi^{3}} \frac{4 R}{14} \frac{48}{2^{5} 6} \frac{T_{0}^{2}}{a}\left(k_{\text {eq }} \eta_{r}\right)^{2} \ell_{\mathrm{eq}}^{2} \ell_{*} A f_{\mathrm{NL}}^{\text {equil }} \\
& \times \int d \ell_{1} d \ell_{2}\left(1+\vec{\ell}_{1} \cdot \vec{\ell}_{2} / \ell_{1} \ell_{2}\right) e^{1 / 2\left(\ell_{3} / \ell_{*}\right)^{1.2}} \\
& \times \frac{1}{\ell_{1}^{3} \ell_{2}^{3}}\left(\ell_{1}+\ell_{2}-\ell_{3}\right) \\
& \times\left[\vec{\ell}_{1} \cdot \vec{\ell}_{2}-\frac{10}{3} \frac{\left(\overrightarrow{\ell_{3}} \cdot \vec{\ell}_{1}\right)\left(\vec{\ell}_{3} \cdot \vec{\ell}_{2}\right)}{\ell_{3}^{2}}\right],
\end{aligned}
$$

where $\ell_{3}$ is given by (372). The factor 3 in front of this expression comes from cyclic permutations. The integral can be performed numerically and, integrating from a minimum $\ell_{\min } \simeq 1200$ up to $\ell_{\max }=2000$, and by taking $R \simeq 0.3$ when evaluated at recombination, $a \simeq 3, T_{0} \simeq 11,\left(k_{\mathrm{eq}} \eta_{r}\right)^{2} \simeq 26$, $\ell_{\mathrm{eq}}=150, \ell_{*}=750$, we find $F_{\text {rec,equil }} \simeq 9.4 \times 10^{-4}$. Finally, for the entry $F_{\text {rec,rec }}$, we get

$$
\begin{aligned}
& F_{\text {rec,rec }}=\frac{f_{\text {sky }}}{\pi} \frac{1}{(2 \pi)^{2}} \int d^{2} \ell_{1} d^{2} \ell_{2} d^{2} \ell_{3} \delta^{(2)}\left(\vec{\ell}_{123}\right) \\
& \times \frac{B_{\mathrm{rec}}^{2}\left(\ell_{1}, \ell_{2}, \ell_{3}\right)}{6 C\left(\ell_{1}\right) C\left(\ell_{2}\right) C\left(\ell_{3}\right)} \\
& =f_{2}^{2} \frac{f_{\mathrm{sky}}}{2^{5} \pi^{3} 6}\left(\frac{4 R}{14}\right)^{2}\left(\frac{T_{0}^{2}}{a}\right)^{2}\left(k_{\mathrm{eq}} \eta_{r}\right)^{4} \ell_{\mathrm{eq}}^{4} \ell_{*} A \\
& \times\left[3 \int d \ell_{1} d \ell_{2} e^{\left(\ell_{3} / \ell_{*}\right)^{1.2}}\right. \\
& \times \frac{\ell_{3}^{3}}{\ell_{1}^{6} \ell_{2}^{6}}\left(\vec{\ell}_{1} \cdot \vec{\ell}_{2}-\frac{10}{3} \frac{\left(\overrightarrow{\ell_{3}} \cdot \vec{\ell}_{1}\right)\left(\vec{\ell}_{3} \cdot \vec{\ell}_{2}\right)}{\ell_{3}^{2}}\right)^{2} \\
& +6 \int d \ell_{1} d \ell_{2} e^{1 / 2\left(\ell_{3} / \ell_{*}\right)^{1.2}} e^{1 / 2\left(\ell_{2} / \ell_{*}\right)^{1.2}} \\
& \times \frac{1}{\ell_{1}^{6} \ell_{2} \ell_{3}^{2}}\left(\overrightarrow{\ell_{1}} \cdot \overrightarrow{\ell_{2}}-\frac{10}{3} \frac{\left(\overrightarrow{\ell_{3}} \cdot \overrightarrow{\ell_{1}}\right)\left(\overrightarrow{\ell_{3}} \cdot \overrightarrow{\ell_{2}}\right)}{\ell_{3}^{2}}\right) \\
& \left.\times\left(\overrightarrow{\ell_{1}} \cdot \overrightarrow{\ell_{3}}-\frac{10}{3} \frac{\left(\overrightarrow{\ell_{2}} \cdot \overrightarrow{\ell_{3}}\right)\left(\overrightarrow{\ell_{1}} \cdot \overrightarrow{\ell_{2}}\right)}{\ell_{2}^{2}}\right)\right],
\end{aligned}
$$


and we find a value $F_{\text {rec,rec }} \simeq 0.014$. We are now able to compute the entries of inverse of the Fisher matrix, $F_{i j}^{-1}$. In the following, we report our results for the signal-to-noise ratios and the degradation parameters

$$
\begin{aligned}
\left(\frac{S}{N}\right)_{\text {equil }} & =\frac{1}{\sqrt{F_{\text {equil,equil }}^{-1}}} \simeq 12.6 \times 10^{-3} f_{\mathrm{NL}}^{\text {equil }}, \\
\left(\frac{S}{N}\right)_{\text {rec }} & =\frac{1}{\sqrt{F_{\text {rec,rec }}^{-1}}} \simeq 0.1, \\
r_{\text {rec,equil }} & =\frac{F_{\text {rec,equil }}^{-1}}{\sqrt{F_{\text {equil,equil }}^{-1} F_{\text {rec,rec }}^{-1}}} \simeq-0.53, \\
d_{\text {rec }} & =F_{\text {rec,rec } F_{\text {rec,rec }}^{-1} \simeq 1.4} \simeq \\
d_{\text {equil }} & =F_{\text {equil,equil }} F_{\text {equil,equil }}^{-1} \simeq 1.4 .
\end{aligned}
$$

As a confirmation of our expectations, we find that the NG of the type given by (378) has a quite high correlation with an equilateral primordial bispectrum. This translates into a degradation in the minimum detectable value for $f_{\mathrm{NL}}^{\text {equil }}$ with respect to the value given in (374). In fact, from the signalto-noise ratio (388), we find a minimum detectable value of

$$
f_{\mathrm{NL}}^{\text {equil }} \simeq 79
$$

imposing that $(S / N)_{\text {equil }}=1$ with an an increase of $\Delta f_{\mathrm{NL}}^{\text {equil }}=$ $\mathcal{O}(10)$. This corresponds to an increase of the $1-\sigma$ uncertainty on $f_{\mathrm{NL}}^{\text {equil }}$ of $20 \%$ (see Section 8.1 ). (Due to a non-vanishing correlation, $r_{i j}$, $(S / N)$ gets modified from its zero-order value to $(S / N)=(S / N)_{0}\left(1-r_{i j}^{2}\right)^{1 / 2}$, so that the minimum detectable value of $f_{\mathrm{NL}}^{\text {equil }}$ gets shifted by a quantity $\Delta f_{\mathrm{NL}}^{\text {equil }} /\left(f_{\mathrm{NL}}^{\text {equil }}\right)_{0}=$ $\left.\left(1-r_{i j}^{2}\right)^{-1 / 2}-1\right)$.

Following Section 8.1, in order to measure the contamination to the primordial bispectra one can define that effective nonlinearity parameter $f_{\mathrm{NL}}^{\mathrm{con}}$ which minimizes the $\chi^{2}$ defined as

$$
\begin{aligned}
\chi^{2}= & \int d^{2} \ell_{1} d^{2} \ell_{2} d^{2} \ell_{3} \delta^{(2)}\left(\vec{\ell}_{123}\right) \\
& \times \frac{\left(f_{\mathrm{NL}}^{\text {equil }} B_{\mathrm{eq}}\left(\ell_{1}, \ell_{2}, \ell_{3} ; f_{\mathrm{NL}}^{\text {equil }}=1\right)-B_{\mathrm{rec}}\left(\ell_{1}, \ell_{2}, \ell_{3}\right)\right)^{2}}{6 C\left(\ell_{1}\right) C\left(\ell_{2}\right) C\left(\ell_{3}\right)},
\end{aligned}
$$

to find

$$
f_{\mathrm{NL}}^{\text {con }}=\left.\frac{F_{\text {rec,equil }}}{F_{\text {equil,equil }}}\right|_{f_{\mathrm{NL}}^{\text {equil }}=1},
$$

and an analogous expression to compute the contamination to the local primordial bispectrum. In this case we find an effective nonlinearity parameter

$$
f_{\mathrm{NL}}^{\mathrm{cont}} \simeq 5 \text {. }
$$

(Notice that sometimes in the literature one can find also an effective nonlinearity parameter $f_{\mathrm{NL}}^{\text {eff. }}$ defined in such a way that the equilateral (or the local) bispectrum has the same Fisher matrix errors as the recombination bispectrum

$$
f_{\mathrm{NL}}^{\text {eff. }}=\left.\frac{\sqrt{F_{\text {rec,rec }}}}{\sqrt{F_{\text {equil,equil }}}}\right|_{f_{\mathrm{NL}}^{\text {equil }}=1} .
$$

However this is not the proper quantity to define the contamination level to primordial NG; here we are just comparing signal-to-noise ratios, while the contamination $f_{\mathrm{NL}}^{\mathrm{con}}$ defined in (395) contains a somewhat richer information, since we are asking what is the value of equilateral (local) $f_{\mathrm{NL}}$ which best mimics the bispectrum from recombination).

9.3.2. Contamination to Primordial Non-Gaussianity of the Local Type. Similarly, we can compute the Fisher matrix accounting for the NG generated at recombination and the primordial NG of the local type

$$
\left\langle\Phi\left(\mathbf{k}_{1}\right) \Phi\left(\mathbf{k}_{2}\right) \Phi\left(\mathbf{k}_{3}\right)\right\rangle=(2 \pi)^{3} \delta^{(3)}\left(\mathbf{k}_{1}+\mathbf{k}_{2}+\mathbf{k}_{3}\right) B_{\mathrm{loc}}\left(k_{1}, k_{2}, k_{3}\right),
$$

where

$$
B_{\mathrm{loc}}\left(k_{1}, k_{2}, k_{3}\right)=f_{\mathrm{NL}}^{\mathrm{loc}} \cdot 2 A^{2} \cdot\left(\frac{1}{k_{1}^{3} k_{2}^{3}}+\frac{1}{k_{1}^{3} k_{3}^{3}}+\frac{1}{k_{2}^{3} k_{3}^{3}}\right) \text {. }
$$

The bispectrum and the signal-to-noise ratio as defined in (366) have already been computed in the flat-sky approximation in [79]. The result is that $(S / N)_{\text {loc }}^{2}=$ $4 \pi^{-2} f_{\text {sky }}\left(\ell_{*} / \ell_{\min }\right)\left(f_{\mathrm{NL}}^{\text {loc }}\right)^{2} A \ell_{\max }^{2}$, corresponding to a minimum detectable value of $f_{\mathrm{NL}}^{\text {loc }}=\mathcal{O}(7)$ for $\ell_{\max }=2000$ (when other possible sources of NG are ignored). We can compute the off-diagonal entry of the Fisher matrix in a similar way to what we have described in this section, and we get $F_{\text {rec,loc }} \simeq$ $8 \times 10^{-3} f_{\mathrm{NL}}^{\text {loc }}$. Finally, the entry $F_{\text {rec,rec }} \simeq 0.014$ has already been computed above. From inverting the Fisher matrix, we get the following signal-to-noise ratios and the degradation parameters

$$
\begin{aligned}
\left(\frac{S}{N}\right)_{\text {loc }} & =\frac{1}{\sqrt{F_{\text {loc,loc }}^{-1}}} \simeq 14 \times 10^{-2} f_{\mathrm{NL}}^{\mathrm{loc}}, \\
\left(\frac{S}{N}\right)_{\mathrm{rec}} & =\frac{1}{\sqrt{F_{\text {rec,erec }}^{-1}}} \simeq 0.1, \\
r_{\text {rec,loc }} & =\frac{F_{\text {rec,loc }}^{-1}}{\sqrt{F_{\text {loc,loc }}^{-1} F_{\text {rec,rec }}^{-1}}} \simeq-0.44, \\
d_{\text {rec }} & =F_{\text {rec,rec } F_{\text {rec,rec }}^{-1}} \simeq 1.2, \\
d_{\text {loc }} & =F_{\text {equil,equil }} F_{\text {equil,equil }}^{-1} \simeq 1.2 .
\end{aligned}
$$

In particular, from (400) we see that now the minimum detectable value of $f_{\mathrm{NL}}^{\text {loc }}$ remains basically unchanged in the presence of the recombination signal. Similarly, the effective $f_{\mathrm{NL}}^{\mathrm{con}}$ reads

$$
f_{\mathrm{NL}}^{\text {con }}=\left.\frac{F_{\text {rec,loc }}}{F_{\text {loc,loc }}}\right|_{f_{\mathrm{NL}}^{\text {loc }}=1} \simeq 0.3
$$


which is much smaller than the effective nonlinearity parameter (405) for the equilateral case. We have also checked the cross-correlation between the primordial local and equilateral bispectra finding a value of $r_{\text {loc,equil }} \simeq 0.23$, which is in agreement with the value reported in [44]. This reflects the fact that the primordial local and equilateral signals are not fully uncorrelated. The reason is due to the fact that the equilateral and local bispectrum (399) and (361) approach the same shape in the equilateral configuration. This is also the reason why the cross-correlation between the primordial local and recombination bispectra is not so small.

\section{How to Perform a Numerical Analysis of the CMB Bispectrum Produced by Second-Order Perturbations}

In the previous section, through a specific example, we have learned some basic principles to determine a reasonable and quite fast analytic estimate for the contamination to the primordial NG. In this section, we provide all the tools necessary to a full numerical implementation of the secondorder Boltzmann equations which allow to obtain a systematic numerical evaluation of the $\mathrm{CMB}$ angular bispectrum produced by second-order cosmological perturbations. This section is mainly based on the results of [96].

In particular, we will apply this formalism to numerically evaluate the contamination to primordial NG from the second-order fluctuations of the Boltzmann equations that come as products of the first-order perturbations, and ignore the intrinsically second-order terms, or the effects of the perturbed recombination $[63,87,89]$. (At the moment of writing this review the calculations that also include the intrinsically second-order terms and the perturbed recombination are in progress and will be presented in [97]). Here, therefore, we come to the discussion of the second example mentioned in Section 7.1. As anticipated there we will see that, unlike the intrinsically second-order contribution considered in the previous section, the products of the first-order perturbations give a CMB bispectrum that peaks in the squeezed configuration. Therefore, we will study the contamination of the primordial NG of the local type.

\subsection{CMB Bispectrum from Second-Order Perturbations}

10.1.1. Definitions. Again, we expand the temperature fluctuation into the linear (first-order) part and the second-order part as

$$
\frac{\Delta T(\widehat{\mathbf{n}})}{T}=\frac{\Delta T^{(1)}(\widehat{\mathbf{n}})}{T}+\frac{\Delta T^{(2)}(\widehat{\mathbf{n}})}{T}+\cdots
$$

The spherical harmonic coefficients of temperature anisotropy, $a_{\ell m}=T^{-1} \int d^{2} \hat{\mathbf{n}} Y_{\ell m}^{*}(\widehat{\mathbf{n}}) \Delta T(\hat{\mathbf{n}})$, are therefore expanded as

$$
a_{\ell m}=a_{\ell m}^{(1)}+a_{\ell m}^{(2)}+\cdots .
$$

Recall that to expand the Boltzmann equation up to the second order in perturbations, we had to expand the distribution function,

$$
f(\mathbf{x}, p, \widehat{\mathbf{n}}, \eta)=2\left[\exp \left\{\frac{p}{T(\eta) e^{\Theta(\mathbf{x}, \hat{\mathbf{n}}, \eta)}}\right\}-1\right]^{-1}
$$

up to the second order in perturbations: $\Theta=\Theta^{(1)}+\Theta^{(2)} / 2+$ $\cdots$, and accordingly $f=f^{(0)}+f^{(1)}+f^{(2)} / 2+\cdots$. Notice that here $\Theta$ does not depend on $p$. This means that in the following, we will just account for those contributions that do not come from terms related to spectral distortions. Such terms will be accounted for in the full computation of [97]. We can then compute the fractional perturbation in photon's energy density at the $i$-th order in perturbations, $\Delta^{(i)}$, by multiplying $f^{(i)}$ by $p$, and integrating over $p^{2} d p$,

$$
\Delta^{(i)} \equiv \frac{\int d p p^{3} f^{(i)}}{\int d p p^{3} f^{(0)}}
$$

At the linear order, we recovered the usual relation between the linear fractional temperature fluctuation, $\Theta^{(1)}=$ $\Delta T^{(1)} / T$, and the linear fractional energy density perturbation, $\Delta^{(1)}=\delta \rho_{\gamma}^{(1)} / \rho_{\gamma}$, that is, $\Delta^{(1)}=4 \Theta^{(1)}$. At the second order, we have

$$
\Delta^{(2)}=4 \Theta^{(2)}+16\left[\Theta^{(1)}\right]^{2},
$$

which is related to the second-order temperature fluctuation as

$$
\begin{aligned}
\frac{\Delta T^{(2)}}{T} & =\frac{1}{8}\left(\Delta^{(2)}-\left\langle\Delta^{(2)}\right\rangle\right)-\frac{3}{2}\left(\left[\Theta^{(1)}\right]^{2}-\left\langle\left[\Theta^{(1)}\right]^{2}\right\rangle\right) \\
& =\frac{1}{2}\left(\Theta^{(2)}-\left\langle\Theta^{(2)}\right\rangle+\left[\Theta^{(1)}\right]^{2}-\left\langle\left[\Theta^{(1)}\right]^{2}\right\rangle\right),
\end{aligned}
$$

where we have subtracted the average of the temperature fluctuation so that the average of $\Delta T^{(2)} / T$ vanishes. Notice that the temperature we are interested in is the temperature of the blackbody that has the same energy as the actual distribution, see for a discussion on this [66].

We compute $a_{\ell m}^{(2)}$ from $\Delta T^{(2)} / T$ using

$$
\begin{aligned}
& a_{\ell m}^{(2)} \\
& =\int d^{2} \hat{\mathbf{n}} Y_{\ell m}^{*}(\widehat{\mathbf{n}}) \frac{\Delta T}{T} \\
& =\tilde{a}_{\ell m}^{(2)}-\frac{3}{2} \sum_{\ell^{\prime} m^{\prime}} \sum_{\ell^{\prime \prime} m^{\prime \prime}}(-1)^{m} g_{\ell \ell^{\prime} \ell^{\prime \prime}}^{-m m^{\prime} m^{\prime \prime}} \\
& \quad \times\left(a_{\ell^{\prime} m^{\prime}}^{(1)} a_{\ell^{\prime \prime} m^{\prime \prime}}^{(1)}-\left\langle a_{\ell^{\prime} m^{\prime}}^{(1)} a_{\ell^{\prime \prime} m^{\prime \prime}}^{(1)}\right\rangle\right),
\end{aligned}
$$


where we define

$$
\begin{aligned}
\tilde{a}_{\ell m}^{(2)} \equiv & \frac{1}{8} \int d^{2} \widehat{\mathbf{n}} Y_{\ell m}^{*}(\widehat{\mathbf{n}})\left(\Delta^{(2)}(\widehat{\mathbf{n}})-\left\langle\Delta^{(2)}(\widehat{\mathbf{n}})\right\rangle\right), \\
g_{\ell_{1} \ell_{2} \ell_{3}}^{m_{1} m_{2} m_{3}} & \equiv \int d^{2} \widehat{\mathbf{n}} Y_{\ell_{1} m_{1}}(\widehat{\mathbf{n}}) Y_{\ell_{2} m_{2}}(\widehat{\mathbf{n}}) Y_{\ell_{3} m_{3}}(\widehat{\mathbf{n}}) \\
= & \sqrt{\frac{\left(2 \ell_{1}+1\right)\left(2 \ell_{2}+1\right)\left(2 \ell_{3}+1\right)}{4 \pi}} \\
& \times\left(\begin{array}{ccc}
\ell_{1} & \ell_{2} & \ell_{3} \\
0 & 0 & 0
\end{array}\right)\left(\begin{array}{ccc}
\ell_{1} & \ell_{2} & \ell_{3} \\
m_{1} & m_{2} & m_{3}
\end{array}\right) .
\end{aligned}
$$

Here, the matrix is the Wigner $3 j$ symbol.

The CMB angular-averaged bispectrum, $B_{\ell_{1} \ell_{2} \ell_{3}}$, is related to the ensemble average of $a_{\ell_{1} m_{1}} a_{\ell_{2} m_{2}} a_{\ell_{3} m_{3}}$ as

$$
B_{\ell_{1} \ell_{2} \ell_{3}} \equiv \sum_{\text {all } m}\left(\begin{array}{ccc}
\ell_{1} & \ell_{2} & \ell_{3} \\
m_{1} & m_{2} & m_{3}
\end{array}\right)\left\langle a_{\ell_{1} m_{1}} a_{\ell_{2} m_{2}} a_{\ell_{3} m_{3}}\right\rangle
$$

This definition guarantees rotational invariance for the bispectrum, and the Wigner $3 j$ symbol ensures that the bispectrum must satisfy triangle conditions: $\ell_{i}-\ell_{j} \mid \leq \ell_{k} \leq$ $\ell_{i}+\ell_{j}$ for all permutations of indices, and selection rules: $m_{1}+m_{2}+m_{3}=0$.

The ensemble average is given by

$$
\begin{aligned}
& \left\langle a_{\ell_{1} m_{1}} a_{\ell_{2} m_{2}} a_{\ell_{3} m_{3}}\right\rangle \\
& =\left\langle a_{\ell_{1} m_{1}}^{(1)} a_{\ell_{2} m_{2}}^{(1)} a_{\ell_{3} m_{3}}^{(2)}\right\rangle+\text { cyclic } \\
& =\left\langle a_{\ell_{1} m_{1}}^{(1)} a_{\ell_{2} m_{2}}^{(1)} \tilde{a}_{\ell_{3} m_{3}}^{(2)}\right\rangle-\frac{3}{2} \sum_{\ell_{3^{\prime} m_{3^{\prime}} \ell_{3^{\prime \prime}} m_{3^{\prime \prime}}}}(-1)^{m_{3}} g_{\ell_{3} \ell_{3^{\prime}} \ell_{3^{\prime \prime}}}^{-m_{3} m_{3^{\prime}} m_{3^{\prime \prime}}} \\
& \quad \times\left(\left\langle a_{\ell_{1} m_{1}}^{(1)} a_{\ell_{2} m_{2}}^{(1)} a_{\ell_{3^{\prime}} m_{3^{\prime}}}^{(1)} a_{\ell_{3^{\prime \prime}} m_{3^{\prime \prime}}}^{(1)}\right\rangle\right. \\
& \left.\quad-\left\langle a_{\ell_{1} m_{1}}^{(1)} a_{\ell_{2} m_{2}}^{(1)}\right\rangle\left\langle a_{\ell_{3^{\prime} m_{3^{\prime}}}^{(1)}}^{(1)} a_{\ell_{3^{\prime \prime}} m_{3^{\prime \prime}}}^{(1)}\right\rangle\right)+ \text { cyclic, }
\end{aligned}
$$

where cyclic means that we have to sum the cyclic permutations of $(416)$ for indices $(1,2,3) \rightarrow(3,1,2) \rightarrow(2,3,1)$.

As we assume that $a_{\ell m}^{(1)}$ 's are Gaussian random variables, the four-point function of $a_{\ell m}^{(1)}$ 's in (416) is given by the sum of products of all possible pairs. Each pair gives the angular power spectrum, $C_{\ell}$ :

$$
\left\langle a_{\ell m}^{(1)} a_{\ell^{\prime} m^{\prime}}^{(1)}\right\rangle=(-1)^{m} C_{\ell} \delta_{\ell \ell^{\prime}} \delta_{-m m^{\prime}}
$$

We obtain

$$
\begin{aligned}
& \left\langle a_{\ell_{1} m_{1}}^{(1)} a_{\ell_{2} m_{2}}^{(1)} a_{\ell_{3^{\prime}} m_{3^{\prime}}}^{(1)} a_{\ell_{3^{\prime \prime}} m_{3^{\prime \prime}}}^{(1)}\right\rangle-\left\langle a_{\ell_{1} m_{1}}^{(1)} a_{\ell_{2} m_{2}}^{(1)}\right\rangle\left\langle a_{\ell_{3^{\prime}} m_{3^{\prime}}}^{(1)} a_{\ell_{3^{\prime \prime}} m_{3^{\prime \prime}}}^{(1)}\right\rangle \\
& =(-1)^{m_{1}+m_{2}} C_{\ell_{1}} C_{\ell_{2}}\left[\delta_{\ell_{1} \ell_{3^{\prime}}} \delta_{-m_{1} m_{3^{\prime}}} \delta_{\ell_{2} \ell_{3^{\prime \prime}}} \delta_{-m_{2} m_{3^{\prime \prime}}}+(1 \longleftrightarrow 2)\right] .
\end{aligned}
$$

Substituting the right hand side of equation (418) for the second term of equation (416), and using $\ell_{1}+\ell_{2}+\ell_{3}=$ even, we obtain the angular averaged bispectrum,

$$
B_{\ell_{1} \ell_{2} \ell_{3}}=\widetilde{B}_{\ell_{1} \ell_{2} \ell_{3}}-3 I_{\ell_{1} \ell_{2} \ell_{3}}\left(C_{\ell_{1}} C_{\ell_{2}}+\text { cyclic }\right) \text {, }
$$

where we have defined the quantities,

$$
\begin{aligned}
I_{\ell_{1} \ell_{2} \ell_{3}} & \equiv \sqrt{\frac{\left(2 \ell_{1}+1\right)\left(2 \ell_{2}+1\right)\left(2 \ell_{3}+1\right)}{4 \pi}}\left(\begin{array}{ccc}
\ell_{1} & \ell_{2} & \ell_{3} \\
0 & 0 & 0
\end{array}\right), \\
\widetilde{B}_{\ell_{1} \ell_{2} \ell_{3}} & =\sum_{\text {all } m}\left(\begin{array}{ccc}
\ell_{1} & \ell_{2} & \ell_{3} \\
m_{1} & m_{2} & m_{3}
\end{array}\right)\left\langle a_{\ell_{1} m_{1}}^{(1)} a_{\ell_{2} m_{2}}^{(1)} \tilde{a}_{\ell_{3} m_{3}}^{(2)}\right\rangle+\text { cyclic. }
\end{aligned}
$$

10.1.2. The Second-Order CMB Radiation Transfer Function. In this section, we show how it is possible to define in a rigorous way the radiation transfer function for $\mathrm{CMB}$ anisotropies at second-order in the perturbations. It is a generalization of the well-known quantity used at linearorder and as such it allows us to develop a systematic numerical analysis of the angular bispectrum produced by second-order perturbations in the very same way as at linearorder various numerical codes are nowadays available for the computation of the CMB power spectrum, such as CMBFAST or CAMB.

The starting point is the Boltzmann equation that governs the evolution of $\Delta^{(1)}(k, \mu, \eta)$ and $\Delta^{(2)}(\mathbf{k}, \hat{\mathbf{n}}, \eta)$, where $\mu=\hat{k} \cdot \hat{n}$ and $\mathbf{n}$ is the direction of propagation of photons. Note that for the linear perturbation there is azimuthal symmetry such that $\Delta^{(1)}$ depends only on the angle between $\mathbf{k}$ and $\mathbf{n}$; however, for the second-order perturbation there is no such symmetry. We write again the Boltzmann equations in Fourier space

$$
\begin{aligned}
& \Delta^{(1)^{\prime}}+i k \mu \Delta^{(1)}-\tau^{\prime} \Delta^{(1)}=S^{(1)}(k, \mu, \eta), \\
& \Delta^{(2)^{\prime}+i k \mu \Delta^{(2)}-\tau^{\prime} \Delta^{(2)}}=S^{(2)}(\mathbf{k}, \hat{\mathbf{n}}, \eta),
\end{aligned}
$$

where the primes denote derivatives with respect to the conformal time $\partial / \partial \eta, S^{(1)}$ and $S^{(2)}$ are the source functions at the first and the second orders, respectively, and $\tau^{\prime}$ is the differential optical depth which is defined by using the mean electron number density, $\bar{n}_{e}$, the Thomson scattering crosssection, $\sigma_{T}$, and the scale factor, $a$, as

$$
\tau^{\prime}=-\bar{n}_{e} \sigma_{T} a
$$

We expand again the angular dependence of $\Delta^{(i)}$ as

$$
\Delta_{\ell m}^{(i)}(\mathbf{k}, \eta)=i^{\ell} \sqrt{\frac{2 \ell+1}{4 \pi}} \int d^{2} \widehat{\mathbf{n}} Y_{\ell m}^{*}(\widehat{\mathbf{n}}) \Delta^{(i)}(\mathbf{k}, \widehat{\mathbf{n}}, \eta),
$$

and that of the source terms as

$$
S_{\ell m}^{(i)}(\mathbf{k}, \eta)=i^{\ell} \sqrt{\frac{2 \ell+1}{4 \pi}} \int d^{2} \widehat{\mathbf{n}} Y_{\ell m}^{*}(\widehat{\mathbf{n}}) S^{(i)}(\mathbf{k}, \widehat{\mathbf{n}}, \eta),
$$

where $i=1,2$. 
The source functions relate the observed $a_{\ell m}$ 's to the primordial curvature perturbations in comoving gauge, $\zeta(\mathbf{k})$. The relations contain the linear radiation transfer function, $g_{\ell}(k)$, and the second-order radiation transfer function, $F_{\ell m}^{\ell^{\prime} m^{\prime}}(k)$, and are given by

$$
\begin{aligned}
a_{\ell m}^{(1)}= & 4 \pi(-i)^{\ell} \int \frac{d^{3} k}{(2 \pi)^{3}} g_{\ell}(k) Y_{\ell m}^{*}(\widehat{\mathbf{k}}) \zeta(\mathbf{k}), \\
\tilde{a}_{\ell m}^{(2)}= & \frac{4 \pi}{8}(-i)^{l} \int \frac{d^{3} k}{(2 \pi)^{3}} \int \frac{d^{3} k^{\prime}}{(2 \pi)^{3}} \int d^{3} k^{\prime \prime \delta^{(3)}}\left(\mathbf{k}^{\prime}, \mathbf{k}^{\prime \prime}, \mathbf{k}\right) \\
& \times \sum_{\ell^{\prime} m^{\prime}} F_{\ell m}^{\ell^{\prime} m^{\prime}}\left(\mathbf{k}^{\prime}, \mathbf{k}^{\prime \prime}, \mathbf{k}\right) Y_{\ell^{\prime} m^{\prime}}^{*}(\hat{\mathbf{k}}) \zeta\left(\mathbf{k}^{\prime}\right) \zeta\left(\mathbf{k}^{\prime \prime}\right) .
\end{aligned}
$$

The linear transfer function is given by

$$
\begin{aligned}
g_{\ell}(k)=\int_{0}^{\eta_{0}} d \eta e^{-\tau}[ & S_{00}^{(1)}(k, \eta)+S_{10}^{(1)}(k, \eta) \frac{d}{d u}+S_{20}^{(1)}(k, \eta) \\
& \left.\times\left(\frac{3}{2} \frac{d^{2}}{d u^{2}}+\frac{1}{2}\right)\right] j_{\ell}(u),
\end{aligned}
$$

where $u \equiv k\left(\eta_{0}-\eta\right)$ and $S_{\ell m}^{(1)}$ is the standard linear source function

$$
\begin{gathered}
S_{00}^{(1)}(k, \eta)=4 \Psi^{(1)^{\prime}}(k, \eta)-\tau^{\prime} \Delta_{0}^{(1)}(k, \eta), \\
S_{10}^{(1)}(k, \eta)=4 k \Phi^{(1)}(k, \eta)-4 \tau^{\prime} v_{0}^{(1)}(k, \eta), \\
S_{20}^{(1)}(k, \eta)=\frac{\tau^{\prime}}{2} \Delta_{2}^{(1)}(k, \eta),
\end{gathered}
$$

where $\Phi^{(1)}(k, \eta)$ and $\Psi^{(1)}(k, \eta)$ are the metric perturbations at the linear order in the longitudinal gauge and $\Delta_{0}^{(1)}(k, \eta)$, $\Delta_{1}^{(1)}(k, \eta)$, and $\Delta_{2}^{(1)}(k, \eta)$ are the coefficients of the expansion in Legendre polynomials of $\Delta^{(1)}(k, \mu, \eta)$. These coefficients $\Delta_{\ell}^{(1)}(k, \eta)$ are related to $\Delta_{\ell m}^{(1)}(423)$ via

$$
\Delta_{\ell m}^{(1)}(\mathbf{k})=i^{\ell} \sqrt{\frac{4 \pi}{2 \ell+1}} Y_{\ell m}^{*}(\hat{\mathbf{k}}) \Delta_{\ell}^{(1)}(\mathbf{k})(2 \ell+1) .
$$

The first-order velocity perturbation, $v_{0}^{(1)}(k, \eta)$, is the irrotational part of the baryon velocity defined by $\mathbf{v}(\mathbf{k})=$ $-i v_{0}(k) \hat{\mathbf{k}}$.

The new piece, the second-order radiation transfer function, is the line-of-sight integral of the second-order source terms in the Boltzmann equation:

$$
\begin{aligned}
& F_{\ell m}^{\ell^{\prime} m^{\prime}}\left(\mathbf{k}^{\prime}, \mathbf{k}^{\prime \prime}, \mathbf{k}\right) \\
& =i^{\ell} \sum_{\lambda \mu}(-1)^{m}(-i)^{\lambda-\ell^{\prime}} g_{\ell \ell^{\prime} \lambda}^{-m m^{\prime} \mu} \sqrt{\frac{4 \pi}{2 \lambda+1}} \\
& \quad \times \int_{0}^{\eta_{0}} d \eta e^{-\tau} \delta_{\lambda \mu}^{(2)}\left(\mathbf{k}^{\prime}, \mathbf{k}^{\prime \prime}, \mathbf{k}, \eta\right) j_{\ell^{\prime}}\left[k\left(\eta-\eta_{0}\right)\right] .
\end{aligned}
$$

Here, we have introduced a new function, $f_{\ell m}^{(2)}\left(\mathbf{k}^{\prime}, \mathbf{k}^{\prime \prime}, \mathbf{k}, \eta\right)$, which is defined by the following equation:

$$
\begin{aligned}
S_{\ell m}^{(2)}(\mathbf{k}, \eta)= & \int \frac{d \mathbf{k}^{\prime}}{(2 \pi)^{3}} d \mathbf{k}^{\prime \prime} \delta^{(3)}\left(\mathbf{k}^{\prime}, \mathbf{k}^{\prime \prime}, \mathbf{k}\right) \oint_{\ell m}^{(2)} \\
& \times\left(\mathbf{k}^{\prime}, \mathbf{k}^{\prime \prime}, \mathbf{k}, \eta\right) \zeta\left(\mathbf{k}^{\prime}\right) \zeta\left(\mathbf{k}^{\prime \prime}\right) .
\end{aligned}
$$

Basically, $f_{\ell m}^{(2)}\left(\mathbf{k}^{\prime}, \mathbf{k}^{\prime \prime}, \mathbf{k}, \eta\right)$ is the second-order source function divided by $\zeta\left(\mathbf{k}^{\prime}\right) \zeta\left(\mathbf{k}^{\prime \prime}\right)$. The explicit expression of the second-order source function can be be read from (134).

Using (425), we calculate the first term in (419), $\widetilde{B}_{\ell_{1} \ell_{2} \ell_{3}}$, as follows:

$$
\begin{aligned}
& \left\langle a_{\ell_{1} m_{1}}^{(1)} a_{\ell_{2} m_{2}}^{(1)} \tilde{a}_{\ell_{3} m_{3}}^{(2)}\right\rangle \\
& =\frac{(-i)^{\ell_{1}+\ell_{2}+\ell_{3}}}{(2 \pi)^{3}} \sum_{\ell_{3} M_{3}} \prod_{i} \int d^{3} k_{i} \delta^{(3)}\left(\mathbf{k}_{123}\right) Y_{\ell_{1} m_{1}}^{*}\left(\hat{\mathbf{k}}_{1}\right) \\
& \quad \times Y_{\ell_{2} m_{2}}^{*}\left(\hat{\mathbf{k}}_{2}\right) Y_{\ell_{3} M_{3}}^{*}\left(\hat{\mathbf{k}}_{3}\right) g_{\ell_{1}}\left(k_{1}\right) g_{\ell_{2}}\left(k_{2}\right) P_{\zeta}\left(k_{1}\right) P_{\zeta}\left(k_{2}\right) \\
& \quad \times\left\{F_{\ell_{3} m_{3}}^{\ell_{3} M_{3}}\left(\mathbf{k}_{1}, \mathbf{k}_{2}, \mathbf{k}_{3}\right)+F_{\ell_{3} m_{3}}^{\ell_{3} M_{3}}\left(\mathbf{k}_{2}, \mathbf{k}_{1}, \mathbf{k}_{3}\right)\right\},
\end{aligned}
$$

where, as in the previous section, we will sometimes use the notation $\mathbf{k}_{123}=\mathbf{k}_{1}+\mathbf{k}_{2}+\mathbf{k}_{3} . P_{\zeta}(k)$ is the power spectrum of $\zeta$ given by the usual definition

$$
\left\langle\zeta\left(\mathbf{k}_{1}\right)\right\rangle=0, \quad\left\langle\zeta\left(\mathbf{k}_{1}\right) \zeta\left(\mathbf{k}_{2}\right)\right\rangle=(2 \pi)^{3} \delta^{(3)}\left(\mathbf{k}_{1}+\mathbf{k}_{2}\right) P_{\zeta}\left(k_{1}\right) .
$$

In order to perform the integral over angles, $\hat{\mathbf{k}}$, we expand the three-dimensional $\delta$-function using Rayleigh's formula,

$$
\begin{aligned}
& \delta^{(3)}\left(\mathbf{k}_{1}+\mathbf{k}_{2}+\mathbf{k}_{3}\right)
\end{aligned}
$$

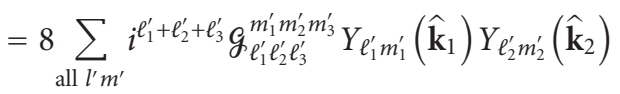

$$
\begin{aligned}
& \times Y_{\ell_{3}^{\prime} m_{3}^{\prime}}\left(\hat{\mathbf{k}}_{3}\right) \int d r r^{2} j_{\ell_{1}^{\prime}}\left(r k_{1}\right) j_{\ell_{2}^{\prime}}\left(r k_{2}\right) j_{\ell_{3}^{\prime}}\left(r k_{3}\right),
\end{aligned}
$$

and also expand the angular dependence of $f_{\ell m}^{(2)}\left(\mathbf{k}_{1}, \mathbf{k}_{2}, \mathbf{k}_{3}, \eta\right)$ by introducing the transformed source function, $f_{\lambda_{1} \lambda_{2} \lambda_{3}}^{\mu_{1} \mu_{2} \mu_{3}}\left(k_{1}, k_{2}, k_{3}, \eta\right)$, as

$$
\begin{aligned}
& f_{\lambda_{3} \mu_{3}}^{(2)}\left(\mathbf{k}_{1}, \mathbf{k}_{2}, \mathbf{k}_{3}, \eta\right) \\
& =\sum_{\lambda_{1}, \mu_{1} \lambda_{2}, \mu_{2}}(-i)^{\lambda_{1}+\lambda_{2}} \sqrt{\frac{4 \pi}{2 \lambda_{1}+1}} \sqrt{\frac{4 \pi}{2 \lambda_{2}+1}} \\
& \quad \times 8_{\lambda_{1} \lambda_{2} \lambda_{3}}^{\mu_{1} \mu_{2} \mu_{3}}\left(k_{1}, k_{2}, k_{3}, \eta\right) Y_{\lambda_{1} \mu_{1}}\left(\widehat{\mathbf{k}}_{1}\right) Y_{\lambda_{2} \mu_{2}}\left(\hat{\mathbf{k}}_{2}\right) .
\end{aligned}
$$

This result shows that $\rho_{\lambda_{3} \mu_{3}}^{(2)}\left(\mathbf{k}_{1}, \mathbf{k}_{2}, \mathbf{k}_{3}, \eta\right)=8_{\lambda_{3} \mu_{3}}^{(2)}\left(\mathbf{k}_{1}, \mathbf{k}_{2}, k_{3}, \eta\right)$, and thus $F_{\ell m}^{\ell^{\prime} m^{\prime}}\left(\mathbf{k}_{1}, \mathbf{k}_{2}, k_{3}\right)$ follows (see (429)). 
Now, we can perform the angular integration of (431) to obtain

$$
\begin{aligned}
& \widetilde{B}_{\ell_{1} \ell_{2} \ell_{3}} \\
& =\frac{4}{\pi^{2}}(-i)^{\ell_{1}+\ell_{2}+\ell_{3}} \sum_{\text {all } m \text { all } \ell^{\prime} m^{\prime} \text { all } \lambda \mu} \sum_{\ell^{\prime}+\ell_{2}^{\prime}+\ell_{3}^{\prime}-\lambda_{1}-\lambda_{2}-\lambda_{3}} \\
& \times \sqrt{\frac{4 \pi}{\left(2 \lambda_{1}+1\right)\left(2 \lambda_{2}+1\right)\left(2 \lambda_{3}+1\right)}}\left(\begin{array}{ccc}
\ell_{1} & \ell_{2} & \ell_{3} \\
m_{1} & m_{2} & m_{3}
\end{array}\right) \\
& \times \mathcal{G}_{\ell_{1}^{\prime} \ell_{2}^{\prime} \ell_{3}^{\prime}}^{m_{1}^{\prime} m_{3}^{\prime} m_{3}^{\prime}} \mathcal{G}_{\ell_{1}^{\prime} \ell_{1} \lambda_{1}}^{m_{1}^{\prime}-m_{1} \mu_{1}} g_{\ell_{2}^{\prime} \ell_{2} \lambda_{2}}^{m_{2}^{\prime}-m_{2} \mu_{2}} \mathcal{G}_{\ell_{3}^{\prime} \ell_{3} \lambda_{3}}^{m_{3}^{\prime}-m_{3} \mu_{3}} \\
& \times \int d r r^{2} \prod_{i=1}^{3} \int k_{i}^{2} d k_{i} j_{\ell_{i^{\prime}}}\left(r k_{i}\right) g_{\ell_{1}}\left(k_{1}\right) g_{\ell_{2}}\left(k_{2}\right) P_{\zeta}\left(k_{1}\right) P_{\zeta}\left(k_{2}\right) \\
& \times i^{\ell_{3}+\ell_{3}^{\prime}} \int d \eta e^{-\tau}\left\{8_{\lambda_{1} \lambda_{2} \lambda_{3}}^{\mu_{1} \mu_{2} \mu_{3}}\left(k_{1}, k_{2}, k_{3}, \eta\right)+1 \longleftrightarrow 2\right\} \\
& \times j_{\ell_{3}^{\prime}}\left[k_{3}\left(\eta-\eta_{0}\right)\right]+\text { cyclic, }
\end{aligned}
$$

where for brevity we indicate $P_{\lambda_{1} \lambda_{2} \lambda_{3}}=$ $\sqrt{4 \pi /\left[\left(2 \lambda_{1}+1\right)\left(2 \lambda_{2}+1\right)\left(2 \lambda_{3}+1\right)\right]}$, and we have used the following relation of the Wigner $9 j$ symbol,

$$
\begin{aligned}
& (-1)^{\ell_{1}^{\prime}+\ell_{2}^{\prime}+\ell_{3}^{\prime}} \sum_{\text {all } m m^{\prime}}\left(\begin{array}{ccc}
\ell_{1} & \ell_{2} & \ell_{3} \\
m_{1} & m_{2} & m_{3}
\end{array}\right) g_{\ell_{1}^{\prime} \ell_{2}^{\prime} \ell_{3}^{\prime}}^{m_{1}^{\prime} m_{2}^{\prime}} g_{\ell_{1}^{\prime} \ell_{1} \lambda_{1}}^{m_{1}^{\prime}-m_{1} \mu_{1}} \\
& \quad \times g_{\ell_{2}^{\prime} \ell_{2} \lambda_{2}}^{m_{2}^{\prime}-m_{2} \mu_{2}} g_{\ell_{3}^{\prime} \ell_{3} \lambda_{3}}^{m_{3}^{\prime}-m_{3} \mu_{3}} \\
& =(-1)^{R} I_{\ell_{1}^{\prime} \ell_{2}^{\prime} \ell_{3}^{\prime}} I_{\ell_{1} \ell_{1}^{\prime} \lambda_{1}} I_{\ell_{2} \ell_{2}^{\prime} \lambda_{2}} I_{\ell_{3} \ell_{3}^{\prime} \lambda_{3}} \\
& \quad \times\left\{\begin{array}{lll}
\ell_{1} & \ell_{2} & \ell_{3} \\
\ell_{1}^{\prime} & \ell_{2}^{\prime} & \ell_{3}^{\prime} \\
\lambda_{1} & \lambda_{2} & \lambda_{3}
\end{array}\right\}\left(\begin{array}{lll}
\lambda_{1} & \lambda_{2} & \lambda_{3} \\
\mu_{1} & \mu_{2} & \mu_{3}
\end{array}\right)
\end{aligned}
$$

with $R \equiv \ell_{1}+\ell_{2}+\ell_{3}+\ell_{1}^{\prime}+\ell_{2}^{\prime}+\ell_{3}^{\prime}+\lambda_{1}+\lambda_{2}+\lambda_{3}$. The Wigner $9 j$ symbols have the permutation symmetry,

$$
\begin{aligned}
(-1)^{R}\left\{\begin{array}{lll}
\ell_{1} & \ell_{2} & \ell_{3} \\
\ell_{1}^{\prime} & \ell_{2}^{\prime} & \ell_{3}^{\prime} \\
\lambda_{1} & \lambda_{2} & \lambda_{3}
\end{array}\right\} & =\left\{\begin{array}{lll}
\ell_{2} & \ell_{1} & \ell_{3} \\
\ell_{2}^{\prime} & \ell_{1}^{\prime} & \ell_{3}^{\prime} \\
\lambda_{2} & \lambda_{1} & \lambda_{3}
\end{array}\right\}=\left\{\begin{array}{lll}
\ell_{1} & \ell_{3} & \ell_{2} \\
\ell_{1}^{\prime} & \ell_{3}^{\prime} & \ell_{2}^{\prime} \\
\lambda_{1} & \lambda_{3} & \lambda_{2}
\end{array}\right\} \\
& =\left\{\begin{array}{lll}
\ell_{1}^{\prime} & \ell_{2}^{\prime} & \ell_{3}^{\prime} \\
\ell_{1} & \ell_{2} & \ell_{3} \\
\lambda_{1} & \lambda_{2} & \lambda_{3}
\end{array}\right\}=\left\{\begin{array}{lll}
\ell_{1} & \ell_{2} & \ell_{3} \\
\lambda_{1} & \lambda_{2} & \lambda_{3} \\
\ell_{1}^{\prime} & \ell_{2}^{\prime} & \ell_{3}^{\prime}
\end{array}\right\},
\end{aligned}
$$

and the coefficients $I_{\ell_{1}^{\prime} \ell_{2}^{\prime} \ell_{3}^{\prime}}, I_{\ell_{1} \ell_{1}^{\prime} \lambda_{1}}, I_{\ell_{2} \ell_{1}^{\prime} \lambda_{2}}$, and $I_{\ell_{3} \ell_{3}^{\prime} \lambda_{3}}$, ensure $\ell_{1}^{\prime}+$ $\ell_{2}^{\prime}+\ell_{3}^{\prime}=$ even, $\ell_{1}+\ell_{1}^{\prime}+\lambda_{1}=$ even, $\ell_{2}+\ell_{2}^{\prime}+\lambda_{2}=$ even, and $\ell_{3}+$ $\ell_{3}^{\prime}+\lambda_{3}=$ even, respectively, which gives $R=$ even. Hence, the Wigner $9 j$ coefficients are invariant under the permutations.
Finally, we obtain the angular averaged bispectrum,

$$
\begin{aligned}
& \widetilde{B}_{\ell_{1} \ell_{2} \ell_{3}} \\
& =\frac{4}{\pi^{2}} \sum_{\text {all } \ell^{\prime} \lambda} \sqrt{\frac{4 \pi}{\left(2 \lambda_{1}+1\right)\left(2 \lambda_{2}+1\right)\left(2 \lambda_{3}+1\right)}} i^{\ell_{3}-\ell_{3}^{\prime}+R} \\
& \quad \times I_{\ell_{1}^{\prime} \ell_{2}^{\prime} \ell_{3}^{\prime}} I_{\ell_{1} \ell_{1}^{\prime} \lambda_{1}} I_{\ell_{2} \ell_{2}^{\prime} \lambda_{2}} I_{\ell_{3} \ell_{3}^{\prime} \lambda_{3}}\left\{\begin{array}{lll}
\ell_{1} & \ell_{2} & \ell_{3} \\
\ell_{1}^{\prime} & \ell_{2}^{\prime} & \ell_{3}^{\prime} \\
\lambda_{1} & \lambda_{2} & \lambda_{3}
\end{array}\right\} \\
& \quad \times \int d r r^{2} \prod_{i=1}^{2} \int d k_{i} k_{i}^{2} P_{\zeta}\left(k_{i}\right) g_{\ell_{i}}\left(k_{i}\right) j_{\ell_{i}^{\prime}}\left(r k_{i}\right) \int d k_{3} k_{3}^{2} j \ell_{\ell^{\prime}}\left(r k_{3}\right) \\
& \quad \times \int d r^{\prime} e^{-\tau\left(r^{\prime}\right)} j_{\ell_{3}^{\prime}}\left(r^{\prime} k_{3}\right) \delta_{\lambda_{1} \lambda_{2} \lambda_{3}}\left(k_{1}, k_{2}, k_{3}, r^{\prime}\right)+\text { perm. },
\end{aligned}
$$

where $r^{\prime} \equiv\left(\eta_{0}-\eta\right)$ and we have used the relation of the spherical Bessel function, $j_{\ell}(-x)=(-1)^{\ell} j_{\ell}(x)$. We also define the "angular-averaged source function,"

$$
\begin{aligned}
& \&_{\lambda_{1} \lambda_{2} \lambda_{3}}\left(k_{1}, k_{2}, k_{3}, r\right) \\
& \equiv \sum_{\text {all } \mu}\left(\begin{array}{lll}
\lambda_{1} & \lambda_{2} & \lambda_{3} \\
\mu_{1} & \mu_{2} & \mu_{3}
\end{array}\right) \oiint_{\lambda_{1} \lambda_{2} \lambda_{3}}^{\mu_{1} \mu_{2} \mu_{3}}\left(k_{1}, k_{2}, k_{3}, r\right) \\
& =i^{\lambda_{1}+\lambda_{2}} \sqrt{\frac{2 \lambda_{1}+1}{4 \pi}} \sqrt{\frac{2 \lambda_{2}+1}{4 \pi}} \sum_{\text {all } \mu}\left(\begin{array}{lll}
\lambda_{1} & \lambda_{2} & \lambda_{3} \\
\mu_{1} & \mu_{2} & \mu_{3}
\end{array}\right) \\
& \quad \times \int d^{2} \widehat{\mathbf{k}}_{1} d^{2} \widehat{\mathbf{k}}_{2} Y_{\lambda_{1} \mu_{1}}^{*}\left(\hat{\mathbf{k}}_{1}\right) Y_{\lambda_{2} \mu_{2}}^{*}\left(\hat{\mathbf{k}}_{2}\right) \oiint_{\lambda_{3} \mu_{3}}\left(\mathbf{k}_{1}, \mathbf{k}_{2}, r\right),
\end{aligned}
$$

where we have used the inverse relation of (434). Note that cyclic terms in (435) have become permutations because of invariance of the Wigner $9 j$ coefficients under the permutations.

The final analytic formula (438), we have obtained is a general formula which can be applied to any second-order perturbations. The information about the specific secondorder terms is contained in the angular-averaged source term, $\diamond_{\lambda_{1} \lambda_{2} \lambda_{3}}$ (see (439) and (434) for the definition).

For terms that are products of the first-order perturbations, there is indeed a further simplification. One can show that $\delta_{\lambda_{1} \lambda_{2} \lambda_{3}}\left(k_{1}, k_{2}, k_{3}, \eta\right)$ does not depend on $k_{3}$, that is, $\delta_{\lambda_{1} \lambda_{2} \lambda_{3}}\left(k_{1}, k_{2}, k_{3}, \eta\right)=\delta_{\lambda_{1} \lambda_{2} \lambda_{3}}\left(k_{1}, k_{2}, \eta\right)$. This property enables us to integrate (438) over $k_{3}$. We obtain

$$
\begin{aligned}
\widetilde{B}_{\ell_{1} \ell_{2} \ell_{3}}= & \frac{2}{\pi} \sum_{\text {all } l^{\prime} \lambda} \sqrt{\frac{4 \pi}{\left(2 \lambda_{1}+1\right)\left(2 \lambda_{2}+1\right)\left(2 \lambda_{3}+1\right)}} i^{\ell_{3}-\ell_{3}^{\prime}+R} \\
& \times I_{\ell_{1}^{\prime} \ell_{2}^{\prime} \ell_{3}^{\prime}} I_{\ell_{1} \ell_{1}^{\prime} \lambda_{1}} I_{\ell_{2} \ell_{2}^{\prime} \lambda_{2}} I_{\ell_{3} \ell_{3}^{\prime} \lambda_{3}}\left\{\begin{array}{ccc}
\ell_{1} & \ell_{2} & \ell_{3} \\
\ell_{1}^{\prime} & \ell_{2}^{\prime} & \ell_{3}^{\prime} \\
\lambda_{1} & \lambda_{2} & \lambda_{3}
\end{array}\right\} \\
& \times \int d r e^{-\tau} \prod_{i=1}^{2} \int d k_{i} k_{i}^{2} P_{\zeta}\left(k_{i}\right) j_{l_{i^{\prime}}}\left(r k_{i}\right) g_{\ell_{i}} \\
& \times\left(k_{i}\right) s_{\lambda_{1} \lambda_{2} \lambda_{3}}\left(k_{1}, k_{2}, r\right)+\text { perm. }
\end{aligned}
$$


where $r \equiv\left(\eta_{0}-\eta\right), R=\ell_{1}+\ell_{2}+\ell_{3}+\ell_{1}^{\prime}+\ell_{2}^{\prime}+\ell_{3}^{\prime}+\lambda_{1}+\lambda_{2}+\lambda_{3}$, and we have used

$$
\int d k_{3} k_{3}^{2} j_{\ell_{3}^{\prime}}\left(r k_{3}\right) j_{\ell_{3}^{\prime}}\left(r^{\prime} k_{3}\right)=\frac{\pi}{2 r^{2}} \delta\left(r-r^{\prime}\right) .
$$

Finally, by adding the remaining term in the full bispectrum, (419), we obtain

$$
\begin{aligned}
& B_{\ell_{1} \ell_{2} \ell_{3}} \\
& =\frac{2}{\pi} \sum_{\text {all } \ell^{\prime} \lambda} \sqrt{\frac{4 \pi}{\left(2 \lambda_{1}+1\right)\left(2 \lambda_{2}+1\right)\left(2 \lambda_{3}+1\right)}} i^{\ell_{3}-\ell_{3}^{\prime}+R} \\
& \quad \times I_{\ell_{1}^{\prime} \ell_{2}^{\prime} \ell_{3}^{\prime}} I_{\ell_{1} \ell_{1}^{\prime} \lambda_{1}} I_{\ell_{2} \ell_{2}^{\prime} \lambda_{2}} I_{\ell_{3} \ell_{3}^{\prime} \lambda_{3}}\left\{\begin{array}{lll}
\ell_{1} & \ell_{2} & \ell_{3} \\
\ell_{1}^{\prime} & \ell_{2}^{\prime} & \ell_{3}^{\prime} \\
\lambda_{1} & \lambda_{2} & \lambda_{3}
\end{array}\right\} \\
& \quad \times \int d r e^{-\tau} \prod_{i=1}^{2} \int d k_{i} k_{i}^{2} P_{\zeta}\left(k_{i}\right) j_{\ell_{i}^{\prime}}\left(r k_{i}\right) g_{\ell_{i}}\left(k_{i}\right) \delta_{\lambda_{1} \lambda_{2} \lambda_{3}}\left(k_{1}, k_{2}, r\right) \\
& \quad-\frac{3}{2} I_{\ell_{1} \ell_{2} \ell_{3}} C_{\ell_{1}} C_{\ell_{2}}+\text { perm. }
\end{aligned}
$$

The remaining task is to calculate the angular-averaged source term, $\oint_{\lambda_{1} \lambda_{2} \lambda_{3}}\left(k_{1}, k_{2}, k_{3}, r\right)$ according to (439). Up to now our scope has been to offer the reader all the general tools that allow a systematic treatment of the CMB angular bispectrum from second-order perturbations. In the following, we will give just some examples of how such tools can be implemented in order to obtain numerical results about signal-to-noise ratios and contamination to primordial non-Gaussianity.

10.1.3. Source Term: An Example. The explicit expression for $S_{\ell m}^{(2)}(\mathbf{k}, \eta)$ can be obtained from (134). Here, we do not want to report the complete expression $S_{\ell m}^{(2)}(\mathbf{k}, \eta)$ of the source function at second-order, rather, for the goal of this paper, we think it is more instructive to show explicitly how the calculation of $\delta_{\lambda_{1} \lambda_{2} \lambda_{3}}$ proceeds focusing on just one simple example.

Therefore, consider, for example, the term of the secondorder source term from (134) given by

$$
2 \tau^{\prime} \delta_{e}^{(1)} \times \Delta^{(1)}
$$

First, we compute the multipole coefficients of the source term $S_{\ell m}^{(2)}(k)$ from this contribution, as defined in (424). They are given by the convolution

$$
S_{\ell m}^{(2)}(\mathbf{k})=\int \frac{d^{3} k^{\prime}}{(2 \pi)^{3}} 2 \tau^{\prime} \delta_{e}^{(1)}\left(\mathbf{k}-\mathbf{k}^{\prime}\right) \Delta_{\ell m}^{(1)}\left(\mathbf{k}^{\prime}\right) .
$$

Now, for $\Delta_{\ell m}^{(1)}(\mathbf{k})$, we use

$$
\Delta_{\ell m}^{(1)}(\mathbf{k})=i \sqrt{\frac{4 \pi}{2 l+1}} Y_{\ell m}^{*}(\widehat{\mathbf{k}}) \Delta_{\ell}^{(1)}(\mathbf{k})(2 \ell+1),
$$

so that

$$
\begin{aligned}
S_{\ell m}^{(2)}(\mathbf{k})= & \int \frac{d^{3} k_{1}}{(2 \pi)^{3}} \int d^{3} k_{2} \delta^{(3)}\left(\mathbf{k}_{1}+\mathbf{k}_{2}-\mathbf{k}\right) \\
& \times i^{\ell} \sqrt{\frac{4 \pi}{2 \ell+1}}(2 \ell+1) 2 \tau^{\prime} \delta_{\ell}\left(\mathbf{k}_{2}\right) \Delta_{\ell}^{(1)}\left(\mathbf{k}_{1}\right) Y_{\ell m}^{*}\left(\hat{\mathbf{k}}_{1}\right) .
\end{aligned}
$$

We now compute the corresponding "angular-averaged source function" coefficients $\delta_{\lambda_{1} \lambda_{2} \lambda_{3}}\left(k_{1}, k_{2}, r\right)$ defined by (439). From (446) you read the kernel defined in(430)

$$
\begin{aligned}
& \delta_{\ell m}^{(2)}\left(\mathbf{k}_{1}, \mathbf{k}_{2}, \eta\right) \\
& =i^{\ell} \sqrt{\frac{4 \pi}{2 \ell+1}}(2 \ell+1) 2 \tau^{\prime} \delta_{\ell}\left(\mathbf{k}_{2}\right) \Delta_{\ell}^{(1)}\left(\mathbf{k}_{1}\right) Y_{\ell m}^{*}\left(\hat{\mathbf{k}}_{1}\right) .
\end{aligned}
$$

From here

$$
\begin{aligned}
& S_{\lambda_{1} \lambda_{2} \lambda_{3}}^{(2)}\left(k_{1}, k_{2}, r\right) \\
& =i^{\lambda_{1}+\lambda_{2}} \sqrt{\frac{2 \lambda_{1}+1}{4 \pi}} \sqrt{\frac{2 \lambda_{2}+1}{4 \pi}} \sum_{\text {all } \mu}\left(\begin{array}{lll}
\lambda_{1} & \lambda_{2} & \lambda_{3} \\
\mu_{1} & \mu_{2} & \mu_{3}
\end{array}\right) \\
& \times \int d^{2} \widehat{\mathbf{k}}_{1} \int d^{2} \widehat{\mathbf{k}}_{2} Y_{\lambda_{1} \mu_{1}}^{*}\left(\widehat{\mathbf{k}}_{1}\right) Y_{\lambda_{2} \mu_{2}}^{*}\left(\mathbf{k}_{2}\right) S_{\lambda_{3} \mu_{3}}^{(2)}\left(\mathbf{k}_{1}, \mathbf{k}_{2}, r\right), \\
& =i^{\lambda_{1}+\lambda_{2}+\lambda_{3}} \sqrt{\frac{2 \lambda_{1}+1}{4 \pi}} \sqrt{\frac{2 \lambda_{2}+1}{4 \pi}} \sqrt{\frac{4 \pi}{2 \lambda_{3}+1}}\left(2 \lambda_{3}+1\right) \\
& \times \sum_{\text {all } \mu}\left(\begin{array}{lll}
\lambda_{1} & \lambda_{2} & \lambda_{3} \\
\mu_{1} & \mu_{2} & \mu_{3}
\end{array}\right) \int d^{2} \widehat{\mathbf{k}}_{1} \int d^{2} \widehat{\mathbf{k}}_{2} Y_{\lambda_{1} \mu_{1}}^{*}\left(\widehat{\mathbf{k}}_{1}\right) \\
& \times Y_{\lambda_{2} \mu_{2}}^{*}\left(\hat{\mathbf{k}}_{2}\right) Y_{\lambda_{3} \mu_{3}}^{*}\left(\hat{\mathbf{k}}_{2}\right) 2 \tau^{\prime} \delta_{e}\left(k_{1}\right) \Delta_{\lambda_{3}}^{(1)}\left(k_{2}\right) \\
& =i^{\lambda_{2}+\lambda_{3}} \delta_{\lambda_{1} 0} \delta_{\lambda_{2} \lambda_{3}} \sqrt{\frac{2 \lambda_{2}+1}{4 \pi}} \sqrt{\frac{4 \pi}{2 \lambda_{3}+1}}\left(2 \lambda_{3}+1\right) \\
& \times 2 \tau^{\prime} \delta_{e}\left(k_{1}\right) \Delta_{\lambda_{3}}^{(1)}\left(k_{2}\right) \sum_{\mu_{2}}(-1)^{\mu_{2}}\left(\begin{array}{ccc}
0 & \lambda_{2} & \lambda_{2} \\
0 & \mu_{2} & -\mu_{2}
\end{array}\right) \text {, }
\end{aligned}
$$

where we have used $Y_{\ell m}^{*}=(-1)^{-m} Y_{\ell-m}$, and the orthonormality of the spherical harmonics. Using the property of the Wigner $3 j$ symbols

$$
\begin{aligned}
\sum_{\mu_{2}}(-1)^{\mu_{2}}\left(\begin{array}{ccc}
0 & \lambda_{2} & \lambda_{2} \\
0 & \mu_{2} & -\mu_{2}
\end{array}\right) & =\sum_{\mu_{2}} \frac{(-1)^{\lambda_{2}}}{\sqrt{2 \lambda_{2}+1}} \\
& =(-1)^{\lambda_{2}} \sqrt{2 \lambda_{2}+1}
\end{aligned}
$$

we find

$\delta_{\lambda_{1} \lambda_{2} \lambda_{3}}^{(2)}\left(k_{1}, k_{2}, r\right)=2 \tau^{\prime}\left(2 \lambda_{2}+1\right)^{(3 / 2)} \delta_{e}\left(k_{1}\right) \Delta_{\lambda_{3}}^{(1)}\left(k_{2}, \eta\right) \delta_{\lambda_{1} 0} \delta_{\lambda_{2} \lambda_{3}}$.

This term therefore corresponds to coefficients $\delta_{0 \lambda_{2} \lambda_{2}}\left(k_{1}\right.$, $\left.k_{2}, r\right)$. 
In general, the perturbation variables of the source term can be split into two parts (see, e.g., (134)). A part containing perturbations that are intrinsically second-order (these perturbations have superscripts (2), and $\omega_{m}$ and $\chi_{m}$ are also intrinsically second-order). Solving for these terms requires solving the full second-order Boltzmann equations coupled with the Einstein equations.

Another part contains terms that are products of two linear variables, as the example that we have just considered. Evaluation of these terms is much easier than that of the intrinsically second-order terms, as the first-order variables have already been calculated using the standard linearized Boltzmann code such as CMBFAST.

\subsection{Second-Order Bispectrum from Products of the First-Order Terms}

10.2.1. A Worked Example. We shall now focus only on the products of the first-order perturbations and we choose to analyze just some of these contributions to offer the reader an example of the analysis one can perform. We warn the reader that the full analysis is under progress and that the full results will be presented in [97], including the intrinsically second-order perturbations which are equally important, and the contribution from perturbing the recombination history $[63,87,89]$.

For the products of the first-order perturbations, from now on we will consider the following nonzero four cases for the source terms, $\delta_{\lambda_{1} \lambda_{2} \lambda_{3}}$, which have been analyzed in [96] (for notational simplicity we shall omit the superscripts (1)). (The following terms are included in the source term $S_{\ell m}^{(2)}(k, \eta)$ as given in [96, Equation (3.2)]. However notice that that expression does not include all the products of firstorder perturbations; the complete expression for the source term will be given in [97]).

$$
\begin{aligned}
\oiint_{000}= & 4 i \tau^{\prime} v_{0}\left(k_{1}\right) \Delta_{1}\left(k_{2}\right) \\
& +\left[2 \tau^{\prime}\left(\delta_{e}+\Phi\right)\left(k_{1}\right)+8 \Psi^{\prime}\left(k_{1}\right)\right] \Delta_{0}\left(k_{2}\right) \\
\S_{110}= & -\frac{20}{\sqrt{3}} \tau^{\prime} v_{0}\left(k_{1}\right) v_{0}\left(k_{2}\right), \\
\oiint_{101}= & 2 i \sqrt{3}\left\{\tau^{\prime} v_{0}\left(k_{1}\right)\left(4 \delta_{e}+4 \Phi+2 \Delta_{0}-\Delta_{2}\right)\left(k_{2}\right)+4 k_{1} \Phi\left(k_{1}\right)\right. \\
& \left.\times\left(\Delta_{0}-\Psi\right)\left(k_{2}\right)+k_{1} \Delta_{0}\left(k_{1}\right)(\Psi+\Phi)\left(k_{2}\right)\right\} \\
\oiint_{112}= & 14 \sqrt{\frac{10}{3}} \tau^{\prime} v_{0}\left(k_{1}\right) v_{0}\left(k_{2}\right) .
\end{aligned}
$$

In particular, it is recognizable the contribution to $\oint_{000}$ from the example just discussed. From these results we find that $s_{\lambda_{1} \lambda_{2} \lambda_{3}}$ does not depend on $k_{3}$, that is, $s_{\lambda_{1} \lambda_{2} \lambda_{3}}=s_{\lambda_{1} \lambda_{2} \lambda_{3}}\left(k_{1}\right.$, $\left.k_{2}, r\right)$. Note also that $\delta_{011}\left(k_{1}, k_{2}, r\right)=\wp_{101}\left(k_{2}, k_{1}, r\right)$.
10.2.2. Bispectrum from Products of the First-Order Terms. For the four nonvanishing combinations of $\lambda_{1}, \lambda_{2}$, and $\lambda_{3}$ in (451), we rewrite the expression for the bispectrum, (442), as

$$
\begin{aligned}
B_{\ell_{1} \ell_{2} \ell_{3}} & =\sum_{\lambda_{1} \lambda_{2} \lambda_{3}} B_{\ell_{1} \ell_{2} \ell_{3}}^{\left(\lambda_{1}, \lambda_{2}, \lambda_{3}\right)}+B_{\ell_{1} \ell_{2} \ell_{3}}^{C l} \\
& =B_{\ell_{1} \ell_{2} \ell_{3}}^{(0,0,0)}+B_{\ell_{1} \ell_{2} \ell_{3}}^{(1,1,0)}+2 B_{\ell_{1} \ell_{2} \ell_{3}}^{(1,0,1)}+B_{\ell_{1} \ell_{2} \ell_{3}}^{(1,1,2)}+B_{\ell_{1} \ell_{2} \ell_{3}}^{C l},
\end{aligned}
$$

where we have used $B_{\ell_{1} \ell_{2} \ell_{3}}^{(0,1,1)}=B_{\ell_{1} \ell_{2} \ell_{3}}^{(1,0,1)}$, and defined

$$
\begin{aligned}
B_{\ell_{1} \ell_{2} \ell_{3}}^{C l} \equiv & -3 I_{\ell_{1} \ell_{2} \ell_{3}} C_{\ell_{1}} C_{\ell_{2}}+\text { cyclic, } \\
B_{\ell_{1} \ell_{2} \ell_{3}}^{\left(\lambda_{1}, \lambda_{2}, \lambda_{3}\right)} \equiv & \frac{2}{\pi} \sum_{\text {all } l^{\prime}} \sqrt{\frac{4 \pi}{\left(2 \lambda_{1}+1\right)\left(2 \lambda_{2}+1\right)\left(2 \lambda_{3}+1\right)}} i^{\ell_{3}-\ell_{3^{\prime}}+R} \\
& \times I_{\ell_{1}^{\prime} \ell_{2}^{\prime} \ell_{3}^{\prime}} I_{\ell_{1} \ell_{1}^{\prime} \lambda_{1}} I_{\ell_{2} \ell_{2}^{\prime} \lambda_{2}} I_{\ell_{3} \ell_{3}^{\prime} \lambda_{3}}\left\{\begin{array}{lll}
\ell_{1} & \ell_{2} & \ell_{3} \\
\ell_{1}^{\prime} & \ell_{2}^{\prime} & \ell_{3}^{\prime} \\
\lambda_{1} & \lambda_{2} & \lambda_{3}
\end{array}\right\} \\
& \times \int d r e^{-\tau} \prod_{i=1}^{2} \int d k_{i} k_{i}^{2} P_{\zeta}\left(k_{i}\right) j_{l_{i^{\prime}}}\left(r k_{i}\right) g_{l_{i}} \\
& \times\left(k_{i}\right) \delta_{\lambda_{1} \lambda_{2} \lambda_{3}}\left(k_{1}, k_{2}, r\right)+\text { perm. }
\end{aligned}
$$

To proceed further, it turns out to be useful to simplify the expression by introducing the following notation for the integral over $k$ that appears many times:

$$
[x]_{\ell \ell^{\prime}}^{(n)}(r) \equiv \frac{2}{\pi} \int d k k^{2+n} P_{\zeta}(k) j_{\ell^{\prime}}(r k) g_{\ell}(k) x(k, r) .
$$

This function corresponds to the existing functions in the literature in the appropriate limits. For example, for $x(k, r)=$ $\pi / 2$, this function is the same as $\beta_{\ell \ell^{\prime}}^{(n)}(r)$ introduced in [98]. In fact, we find that an order-of-magnitude estimate of $[x]_{\ell \ll{ }^{\prime}}^{(n)}(r)$ is given by $[x]_{\ell \ell^{\prime}}^{(n)}(r) \sim 2 \beta_{\ell \ell^{\prime}}^{(n)}(r) / \pi \times x\left(k=\ell^{\prime} / r, r\right)$ for a smooth function of $x(k, r)$. As $\beta_{\ell \ell^{\prime}}^{(n)}(r)$ is a sharply peaked function at the decoupling epoch, $r=r_{*}$, we find that $[x]_{\ell \ell^{\prime}}^{(n)}(r)$ is also sharply peaked at $r=r_{*}$. With these tools in hand, one can calculate $B_{\ell_{1} \ell_{2} \ell_{3}}^{(0,0)}, B_{\ell_{1} \ell_{2} \ell_{3}}^{(1,1,0)}, B_{\ell_{1} \ell_{2} \ell_{3}}^{(1,0,1)}$, and $B_{\ell_{1} \ell_{2} \ell_{3}}^{(1,1,2)}$. We do not bother here the reader with the details of this computation whose details can be found in [96]. Rather here, we prefer to go straight to the results of the analysis of these bispectra which can be particularly instructive as far as their shape and their contamination to primordial nonGaussianity are concerned.

10.3. Shape and Signal-to-Noise of the Second-Order Bispectrum from Products of the First-Order Terms. One of the motivations for calculating the second-order bispectrum is to see how much the second-order effects in gravity and the photon-baryon fluid contaminate the extraction of the primordial bispectrum. If, for example, the predicted shape of the second-order bispectrum is sufficiently different from that of the primordial bispectrum, then one would hope that the contamination would be minimal. To investigate 


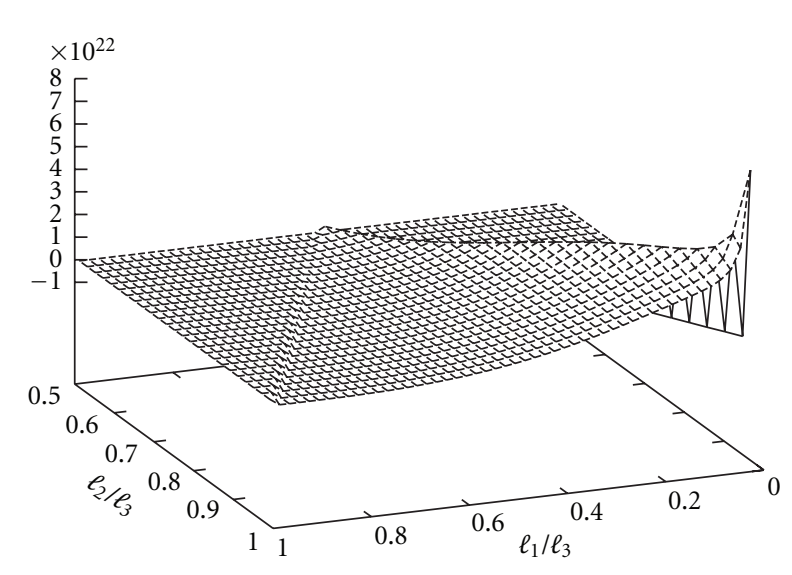

(a)

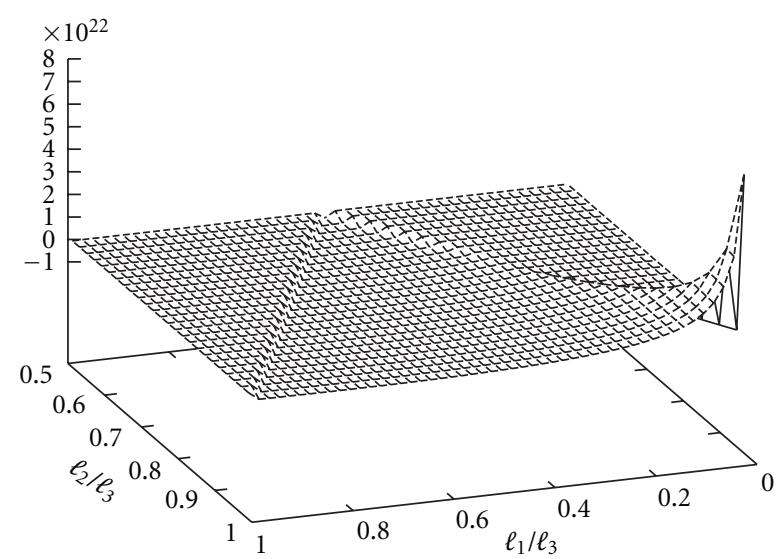

(b)

FIGURE 1: Shape dependence of the second-order bispectrum from products of the first-order terms (top) and that of the local primordial bispectrum (bottom). We show $\ell_{1} \ell_{2}\left\langle a_{\ell_{1} m_{1}}^{(1)} a_{\ell_{2} m_{2}}^{(1)} a_{\ell_{3} m_{3}}^{(2)}\right\rangle\left(g_{\ell_{1} \ell_{2} \ell_{3}}^{m_{1} m_{3}}\right)^{-1} /(2 \pi)^{2} \times 10^{22}$ as a function of $\ell_{1} / \ell_{3}$ and $\ell_{2} / \ell_{3}$ where $\ell_{3}=200$. Both shapes have the largest signals in the squeezed triangles, $\ell_{1} \ll \ell_{2} \approx \ell_{3}$.

this, we shall compare the numerical results of the secondorder bispectrum with the so-called "local" model of the primordial bispectrum.

We extract the first-order perturbations from the CMBFAST code. We use the following cosmological parameters: $\Omega_{\Lambda}=0.72, \Omega_{m}=0.23, \Omega_{b}=0.046, h=0.70$, and assume a power law spectrum, $P_{\zeta} \propto k^{n-4}$, with $n=1$. We determine the decoupling time, $\eta_{*}$, from the peak of the visibility function. In this model, we have $c \eta_{0}=14.9 \mathrm{Gpc}$ and $c \eta_{*}=288 \mathrm{Mpc}$. While the most of the signal is generated in the region of the decoupling epoch, in the low- $\ell$ regime we must also take into account the late time contribution due to the late integrated Sachs-Wolfe effect; thus, we integrate over the line-of-sight, $r$, in the following regions: $c\left(\eta_{0}-5 \eta_{*}\right)<$ $r<c\left(\eta_{0}-0.7 \eta_{*}\right)$ for $\ell>100$, and $0<r<c\left(\eta_{0}-0.7 \eta_{*}\right)$ for $l \leq 100$. The step size is $\Delta r=0.1 \eta_{*}$ around the decoupling epoch, and we use the same time steps used by CMBFAST after the decoupling epoch.

The local primordial bispectrum is given by [43]

$$
B_{\ell_{1} \ell_{2} \ell_{3}}=2 I_{\ell_{1} \ell_{2} \ell_{3}} \int_{0}^{\infty} r^{2} d r b_{\ell_{1}}^{L}(r) b_{\ell_{2}}^{L}(r) b_{\ell_{3}}^{\mathrm{NL}}(r)+\text { cyclic, }
$$

where

$$
\begin{aligned}
b_{\ell}^{L}(r) & \equiv \frac{2}{\pi} \int_{0}^{\infty} k^{2} d k P_{\Phi}(k) g_{T \ell}^{\mathrm{KS}}(k) j_{\ell}(k r), \\
b_{\ell}^{\mathrm{NL}}(r) & \equiv \frac{2}{\pi} \int_{0}^{\infty} k^{2} d k f_{\mathrm{NL}} g_{T \ell}^{\mathrm{KS}}(k) j_{\ell}(k r) .
\end{aligned}
$$

Note that our linear transfer function, $g_{\ell}(k)$, is related to that of [43], $g_{T \ell}^{\mathrm{KS}}(k)$, by $g_{\ell}(k)=(3 / 5) g_{T \ell}^{\mathrm{K} S}(k)$.

Figure 1 shows the shape of the bispectrum generated by the products of the first-order terms $\mathrm{f}$ selected in (451), and compares it to the primordial local bispectrum, for $\ell_{3}=200$. Both shapes (second-order and primordial) have the largest signals in the squeezed triangles, $\ell_{1} \ll \ell_{2} \approx$ $\ell_{3}$. This is an expected result: both the local primordial bispectrum and the second-order bispectrum that we have computed here arise from the products of the first-order terms, also products in position space. However, these two shapes are slightly different when $\ell_{1} / \ell_{3}$ is not so small $\left(\ell_{1} / \ell_{3}=\mathcal{O}(0.1)\right)$ : the ways in which the radiation transfer function (which gives the acoustic oscillations) enters into the bispectrum are different for the products of the firstorder terms and the primordial bispectrum. The primordial bispectrum contains $j_{\ell}\left(k r_{*}\right) g_{\ell}(k)$ whereas the second-order bispectrum contains $j_{\ell}\left(k r_{*}\right) g_{\ell}(k) x\left(k, r_{*}\right)$, where $x=\Delta_{0}$, $v_{0}$, and so forth, also has the oscillations. Therefore, the second-order bispectrum has more interferences between multiple radiation transfer functions. Moreover, the secondorder effects contain derivatives that the local primordial effects do not have, which also makes the details of the two shapes different.

Notice, in particular, that most of these gradients in the source term, (134), are contracted with the direction vector, $\hat{\mathbf{n}}$. There is only one term that has a scalar product of two wave-vectors, $\mathbf{k}_{1} \cdot \mathbf{k}_{2}$, which vanishes in the squeezed limit. The resulting bispectrum, (452), resembles that of a local form, except for the extra powers of $k$ coming from the derivatives. These extra powers of $k$ will affect the scaledependence of the bispectrum, that is, the second-order bispectrum is no longer scale-invariant. Nevertheless, the largest signal of the bispectrum still comes from the squeezed configurations, as the number of extra powers of $k$ from the derivatives in the source term is not large enough to change the fact that we have the largest contribution when one of $k_{1}, k_{2}$, and $k_{3}$ is very small. In other words, schematically the bispectrum looks like $B\left(k_{1}, k_{2}, k_{3}\right) \sim\left(k_{1}^{m_{1}} k_{2}^{m_{1}}\right) /\left(k_{1}^{3} k_{2}^{3}\right)+$ cyclic, where $m_{1}$ and $m_{2}$ are the extra powers of $k$ from the derivatives. Therefore, the largest contribution is in the squeezed configurations as long as $m_{i}<3$. Figure 2 shows the same for $\ell_{3}=1000$. The results are similar to those for $\ell_{3}=200$, but the acoustic oscillations are more clearly visible. 


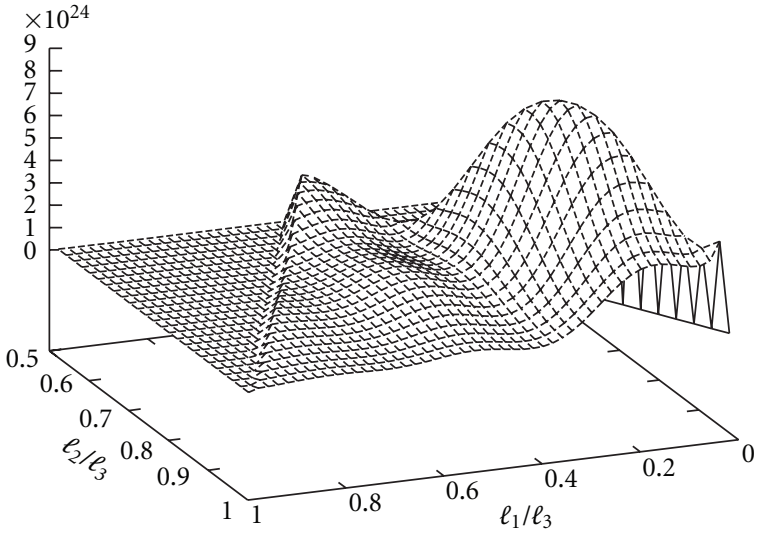

(a)

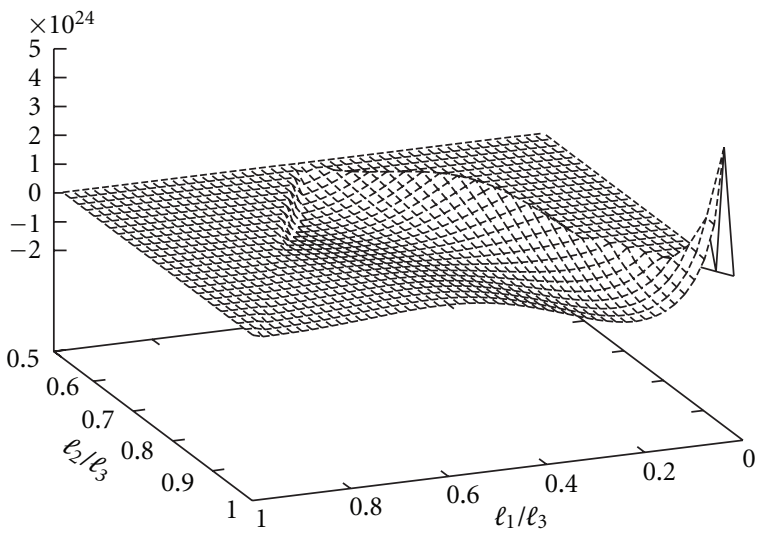

(b)

Figure 2: Same as Figure 1 for $\ell_{3}=1000$. The acoustic oscillations are clearly seen.

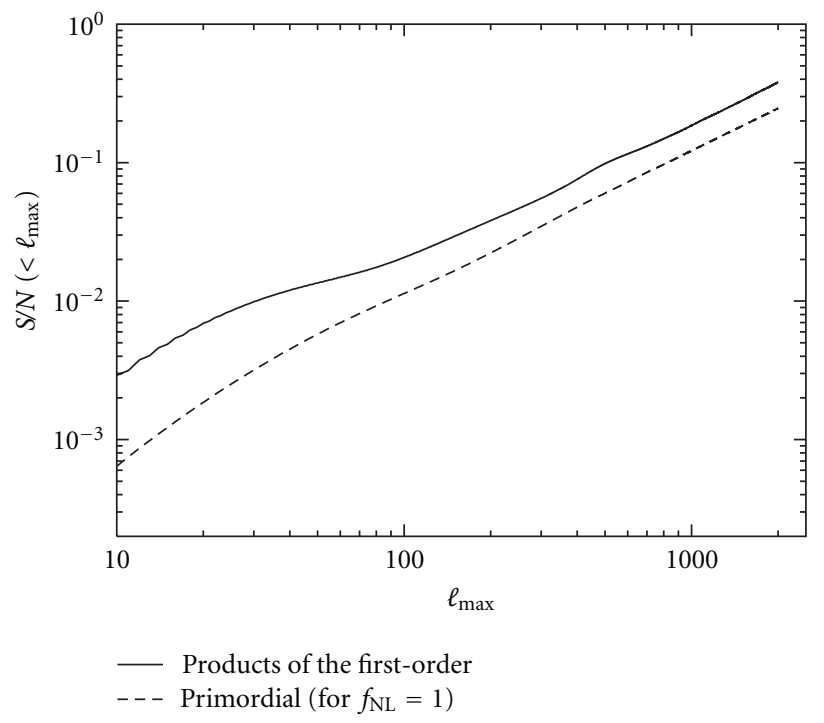

FIGURE 3: Signal-to-noise ratios for the local primordial bispectrum for $f_{\mathrm{NL}}=1$ (dashed), and the second-order bispectrum from the products of the first-order terms (solid), for an ideal full-sky and cosmic-variance-limited (noiseless) experiment.

We can quantify the degree to which the secondorder and the primordial bispectra are correlated, as well as the expected signal-to-noise ratio of the second-order bispectrum, following the general definitions summarized in Section 8. Notice that we are ignoring the noise contribution. In other words, we shall only consider ideal cosmic-variance limited experiments with full sky coverage, which however, at least for the multipole maximum multipole of $l_{\max } \sim 2000$ we will consider, is a good reference for an experiment like Planck (see, e.g., [43]).

The signal-to-noise ratio is given by (356). In Figure 3 we show the cumulative signal-to-noise ratio, summed up to a maximum multipole of $\ell_{\max }$, of the primordial bispectrum, assuming $f_{\mathrm{NL}}=1$ and ignoring the second-order bispectrum, that is, $(S / N)_{\text {prim }}=\left(F_{\text {prim,prim }}\right)^{1 / 2}$, as well as that of the second-order bispectrum, ignoring the primordial bispectrum, that is, $(S / N)_{2 \text { nd }}=\left(F_{2 n d, 2 n d}\right)^{1 / 2}$. In both cases $S / N$ increases roughly as $S / N \propto \ell_{\max }\left(\right.$ or $\propto \sqrt{N_{\text {pix }}}$ where $N_{\text {pix }}$ is the number of independent pixels in the map). A larger contribution to the second-order bispectrum at $\ell \leq$ 50 comes from the terms involving the Integrated SachsWolfe effect. The signal-to-noise ratio of the second-order bispectrum reaches $\sim 0.4$ at $\ell_{\max }=2000$; thus, this signal is undetectable. While our calculation includes the temperature anisotropy only, including polarization would increase the signal-to-noise by a factor of two at most, which would not be enough to push the signal-to-noise above unity.

How similar are the second-order and the primordial bispectra? In Figure 4, we show the cross-correlation coefficient, between the local bispectrum and second-order bispectrum from the products of the first-order terms given in (451). The cross-correlation coefficient (see (357), reaches $\sim 0.5$ for $\ell_{\max }=200$, and the shapes for $\ell_{3}=200$ are shown in Figure 1. After $\ell_{\max }=200$ the correlation weakens, and reaches $\sim 0.35$ at $\ell_{\max }=1000$, and the shapes for $\ell_{3}=$ 1000 are shown in Figure 2. These results show that the second-order bispectrum from the products of the first-order perturbations and the local primordial bispectrum are fairly similar, with a sizable correlation coefficient.

How large is the contamination of the primordial bispectrum? The level of contamination is measured by the effective nonlinearity parameter given by (359).

In Figure 5 , we show $f_{\mathrm{NL}}^{\text {con }}$ as a function of the maximum multipoles, $\ell_{\max }$. We find that $f_{\mathrm{NL}}^{\text {con }}$ reaches the maximum value, $\sim 0.9$, when the correlation coefficient reaches the maximum at $\ell_{\max } \sim 200$, but then decreases to $\sim 0.5$ at $\ell_{\max } \sim$ 2000. Therefore, we conclude that the contamination of the primordial bispectrum due to the second-order bispectrum from the terms in (451) is negligible for CMB experiments.

Finally, one can also calculate the $1-\sigma$ uncertainty of $f_{\mathrm{NL}}, \delta f_{\mathrm{NL}}$, with the second-order bispectrum marginalized over. This is given by $\delta f_{\mathrm{NL}}=\sqrt{\left.(F)_{\text {prim,prim }}^{-1}\right|_{f_{\mathrm{NL}}=1}}$. Figure 6 shows that an increase in the uncertainty of $f_{\mathrm{NL}}$ due to marginalization is totally negligible. 


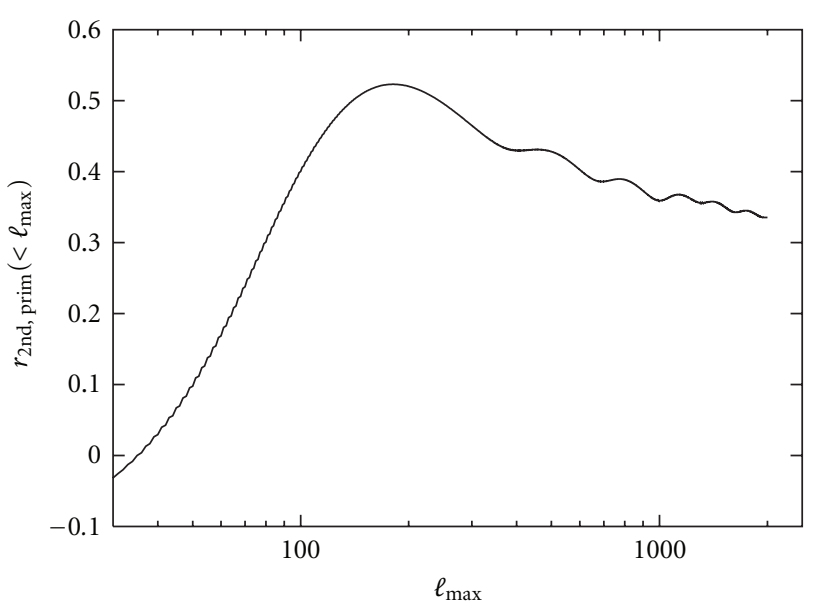

Figure 4: The cross-correlation coefficient between the secondorder bispectrum from the products of the first-order terms and the local primordial bispectrum.

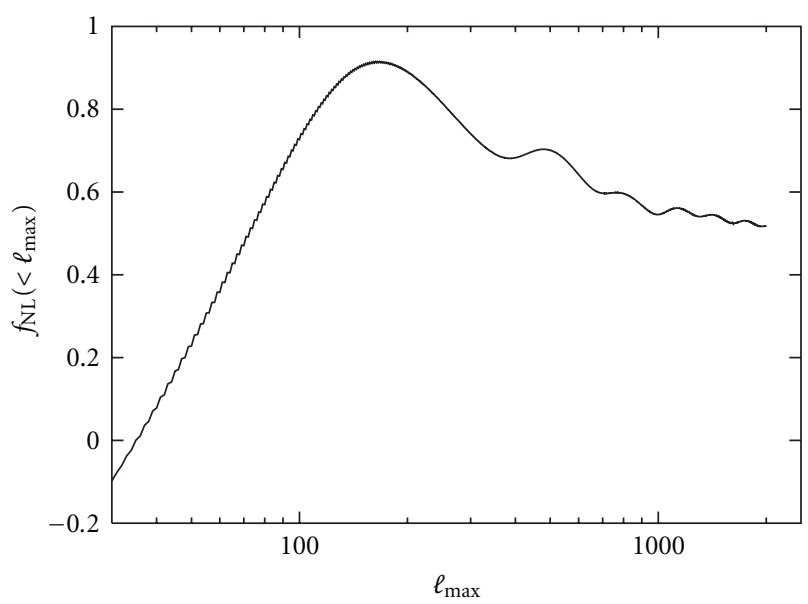

Figure 5: Contamination of the local primordial bispectrum as measured by $f_{\mathrm{NL}}^{\text {con }}(359)$.

\section{Conclusions}

In this paper we have addressed a basic question in cosmology: how a primordial NG propagates into an observable like the CMB anisotropy. Answering this question is fundamental as it will help us in getting some knowledge about the way the primordial cosmological perturbation was generated at the very early stages of the evolution of the universe. In the first sections, we have shown how to set the initial conditions at second-order for the (gauge-invariant) $\mathrm{CMB}$ anisotropy when some source of primordial NG is present. This was more or less straightforward because on large angular scales it is basically gravity which dictates the nonlinear dynamics. On small angular scales, the computation of the second-order effects in the $\mathrm{CMB}$ anisotropy

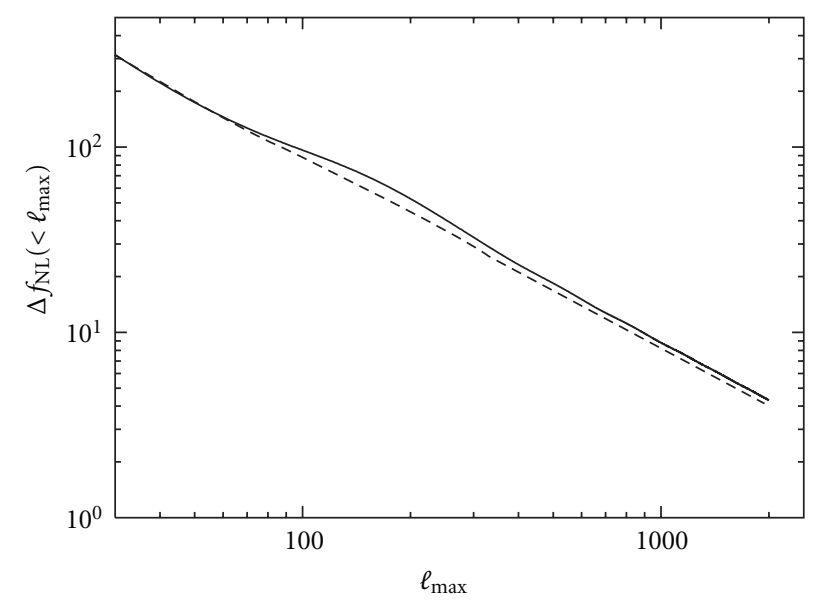

FIGURE 6: Projected uncertainty of $f_{\mathrm{NL}}$ with (dashed) and without (solid) the second-order bispectrum marginalized over.

is far more difficult, as so many sources of nonlinearities appear. In this paper, we have focussed on the study of the second-order effects appearing at the recombination era when the CMB anisotropy is left imprinted. We have shown how to derive the equations which allow to evaluate $\mathrm{CMB}$ anisotropies, by computing the Boltzmann equations describing the evolution of the baryon-photon fluid up to second order. This allows to follow the time evolution of CMB anisotropies (up to second order) on all scales, from the early epoch, when the cosmological perturbations were generated, to the present time, through the recombination era. Through some analytical and simplified example, we have also shown how to estimate the contamination of the recombination secondary effects onto the detection of primordial NG. More refined numerical results confirm our estimates and are also reported here. It goes without saying that this line of research should be pursued until the level of accuracy in the theoretical prediction is reached and is comparable to the one provided by the current or future satellite experiments.

\section{Appendices}

\section{A. Energy-Momentum Tensor}

A.1. Energy-Momentum Tensor for Photons. The energymomentum tensor for photons is defined as

$$
T_{\gamma \nu}^{\mu}=\frac{2}{\sqrt{-g}} \int \frac{d^{3} P}{(2 \pi)^{3}} \frac{P^{\mu} P_{\nu}}{P^{0}} f,
$$

where $g$ is the determinant of the metric (1) and $f$ is the distribution function. We thus obtain 
TABLE 1

\begin{tabular}{|c|c|c|}
\hline Symbol & Definition & Equation \\
\hline$\Phi, \Psi$ & Gravitational potentials in Poisson gauge & $(1)$ \\
\hline$\omega_{i}$ & 2nd-order vector perturbation in Poisson gauge & (1) \\
\hline$\chi_{i j}$ & 2nd-order tensor perturbation in Poisson gauge & (1) \\
\hline$\eta$ & Conformal time & (1) \\
\hline$f$ & Photon distribution function & $(64)$ \\
\hline$g$ & Distribution function for massive particles & $(84) \&(156)$ \\
\hline$f^{(i)}$ & $i$ th order perturbation of the photon distribution function & $(79)$ \\
\hline$f_{\ell m}^{(i)}$ & Moments of the photon distribution function & $(98)$ \\
\hline$C(\mathbf{p})$ & Collision term & $(83) \&(87)$ \\
\hline$p$ & Magnitude of photon momentum $\left(\mathbf{p}=p n^{i}\right)$ & $(59)$ \\
\hline$n^{i}$ & Propagation direction & $(62)$ \\
\hline$\Delta^{(1)}\left(x^{i}, n^{i}, \eta\right)$ & First-order fractional energy photon fluctuations & $(128)$ \\
\hline$\Delta^{(2)}\left(x^{i}, n^{i}, \eta\right)$ & Second-order fractional energy photon fluctuations & $(132)$ \\
\hline$n_{e}$ & Electron number density & $(166)$ \\
\hline$\delta_{e}\left(\delta_{b}\right)$ & Electron (baryon) density perturbation & $(94)$ \\
\hline $\mathbf{k}$ & Wavenumber & $(142)$ \\
\hline$v_{m}$ & Baryon velocity perturbation & $(145) \&(146)$ \\
\hline$v_{\mathrm{CDM}}^{(2) i}$ & Cold dark matter velocity & $(201)$ \\
\hline$v_{\gamma}^{(2) i}$ & Second-order photon velocity & $(187)$ \\
\hline$S_{\ell m}$ & Temperature source term & $(143)$ \\
\hline$\tau$ & Optical depth & $(140)$ \\
\hline $\bar{\rho}_{\gamma}\left(\bar{\rho}_{b}\right)$ & Background photon (baryon) energy density & $(198)$ \\
\hline$B_{\ell_{1}, \ell_{2}, \ell_{3}}$ & $\mathrm{CMB}$ angular-averaged bispectrum & $(353)$ \\
\hline$F_{i j}$ & Fisher matrix for the amplitudes of the bispectra & $(354)$ \\
\hline$f_{\mathrm{NL}}^{\mathrm{con}}$ & contamination to primordial non-Gaussianity & $(359)$ \\
\hline
\end{tabular}

$$
\begin{aligned}
T_{\gamma 0}^{0}= & -\bar{\rho}_{\gamma}\left(1+\Delta_{00}^{(1)}+\frac{\Delta_{00}^{(2)}}{2}\right) \\
T_{\gamma 0}^{i}= & -\frac{4}{3} e^{\Psi+\Phi} \bar{\rho}_{\gamma}\left(v_{\gamma}^{(1) i}+\frac{1}{2} v_{\gamma}^{(2) i}+\Delta_{00}^{(1)} v_{\gamma}^{(1) i}\right) \\
& +\frac{1}{3} \bar{\rho}_{\gamma} e^{\Psi-\Phi} \omega^{i} \\
T_{\gamma j}^{i}= & \bar{\rho}_{\gamma}\left(\Pi_{\gamma j}^{i}+\frac{1}{3} \delta_{j}^{i}\left(1+\Delta_{00}^{(1)}+\frac{\Delta_{00}^{(2)}}{2}\right)\right)
\end{aligned}
$$

where $\bar{\rho}_{\gamma}$ is the background energy density of photons and

$$
\Pi_{\gamma}^{i j}=\int \frac{d \Omega}{4 \pi}\left(n^{i} n^{j}-\frac{1}{3} \delta^{i j}\right)\left(\Delta^{(1)}+\frac{\Delta^{(2)}}{2}\right),
$$

is the quadrupole moment of the photons.

A.2. Energy-Momentum Tensor for Massive Particles. The energy-momentum tensor for massive particles of mass $m$, number density $n$ and degrees of freedom $g_{d}$

$$
T_{m v}^{\mu}=\frac{g_{d}}{\sqrt{-g}} \int \frac{d^{3} Q}{(2 \pi)^{3}} \frac{Q^{\mu} Q_{v}}{Q^{0}} g_{m},
$$

where $g_{m}$ is the distribution function. We obtain

$$
\begin{aligned}
T_{m 0}^{0} & =-\rho_{m}=-\bar{\rho}_{m}\left(1+\delta_{m}^{(1)}+\frac{1}{2} \delta_{m}^{(2)}\right) \\
T_{m 0}^{i} & =-e^{\Psi+\Phi} \rho_{m} v_{m}^{i} \\
& =-e^{\Phi+\Psi} \bar{\rho}_{m}\left(v_{m}^{(1) i}+\frac{1}{2} v_{m}^{(2) i}+\delta_{m}^{(1)} v_{m}^{(1) i}\right) \\
T_{m j}^{i} & =\rho_{m}\left(\delta_{j}^{i} \frac{T_{m}}{m}+v_{m}^{i} v_{m j}\right)=\bar{\rho}_{m}\left(\delta_{j}^{i} \frac{T_{m}}{m}+v_{m}^{(1) i} v_{m j}^{(1)}\right),
\end{aligned}
$$

where $\bar{\rho}_{m}$ is the background energy density of massive particles and we have included the equilibrium temperature $T_{m}$.

\section{B. Solutions of Einstein's Equations in Various Eras}

B.1. Matter-Dominated Era. During the phase in which the CDM is dominating the energy density of the Universe, $a \sim \eta^{2}$ and we may use (34) to obtain an equation for the gravitational potential at first order in perturbation theory 
$\left(\right.$ for which $\left.\Phi^{(1)}=\Psi^{(1)}\right)$

$$
\Phi^{(1)^{\prime \prime}}+3 \mathscr{H} \Phi^{(1)^{\prime}}=0
$$

which has two solutions $\Phi_{+}^{(1)}=$ constant and $\Phi_{-}^{(1)}=\mathscr{H} / a^{2}$. At the same order of perturbation theory, the CDM velocity can be read off from (30)

$$
v^{(1) i}=-\frac{2}{3 \mathscr{H}} \partial^{i} \Phi^{(1)} .
$$

The matter density contrast $\delta^{(1)}$ satisfies the first-order continuity equation

$$
\delta^{(1)^{\prime}}=-\frac{\partial v^{(1) i}}{\partial x^{i}}=-\frac{2}{3 \mathscr{H}} \nabla^{2} \Phi^{(1)}
$$

Going to Fourier space, this implies that

$$
\delta_{k}^{(1)}=\delta_{k}^{(1)}(0)+\frac{k^{2} \eta^{2}}{6} \Phi_{k}^{(1)}
$$

where $\delta_{k}^{(1)}(0)$ is the initial condition in the matter-dominated period.

At second order, using (34) and (32), and the fact that the first-order gravitational potential is constant, we find and equation for the gravitational potential at second-order $\Psi^{(2)}$

$$
\begin{aligned}
\Psi^{(2)^{\prime \prime}}+3 \mathscr{H} \Psi^{(2)^{\prime}} & =S_{m}, \\
S_{m} & =-\partial_{k} \Phi^{(1)} \partial^{k} \Phi^{(1)}+N \\
& =-\partial_{k} \Phi^{(1)} \partial^{k} \Phi^{(1)}+\frac{10}{3} \frac{\partial_{i} \partial^{j}}{\nabla^{2}}\left(\partial_{i} \Phi^{(1)} \partial_{j} \Phi^{(1)}\right),
\end{aligned}
$$

whose solution is

$$
\begin{aligned}
\Psi^{(2)}= & \Psi_{m}^{(2)}(0) \\
& +\int_{0}^{\eta} d \eta^{\prime} \frac{\Phi_{+}^{(1)}(\eta) \Phi_{-}^{(1)}\left(\eta^{\prime}\right)-\Phi_{-}^{(1)}(\eta) \Phi_{+}^{(1)}\left(\eta^{\prime}\right)}{W\left(\eta^{\prime}\right)} S_{m}\left(\eta^{\prime}\right) \\
= & \Psi_{m}^{(2)}(0) \\
& -\frac{1}{14}\left(\partial_{k} \Phi^{(1)} \partial^{k} \Phi^{(1)}-\frac{10}{3} \frac{\partial_{i} \partial^{j}}{\nabla^{2}}\left(\partial_{i} \Phi^{(1)} \partial_{j} \Phi^{(1)}\right)\right) \eta^{2},
\end{aligned}
$$

with $W(\eta)=W_{0} / a^{3}\left(a_{0}=1\right)$ the Wronskian obtained from the corresponding homogeneous equation. In (B.6), $\Psi_{m}^{(2)}(0)$ represents the initial condition (taken conventionally at $\eta \rightarrow$ 0 ) deep in the matter-dominated phase.
From (35), we may compute the vector perturbation in the metric

$$
-\frac{1}{2} \nabla^{2} \omega^{i}=3 \mathscr{H}^{2} \frac{1}{\nabla^{2}} \partial_{j}\left(\partial^{i} \delta^{(1)} v^{(1) j}-\partial^{j} \delta^{(1)} v^{(1) i}\right),
$$

where we have made use of the fact that the vector part of the CDM velocity satisfies the relation $\left(\delta_{j}^{i}-\left(\partial^{i} \partial_{j} / \nabla^{2}\right)\right) v^{(2) i}=$ $-\omega^{i}$.

B.2. Radiation-Dominated Era. We consider a universe dominated by photons and massless neutrinos. The energymomentum tensor for massless neutrinos has the same form as that for photons. During the phase in which radiation is dominating the energy density of the Universe, $a \sim \eta$ and we may combine (29) and (34) to obtain an equation for the gravitational potential $\Psi^{(1)}$ at first order in perturbation theory

$$
\begin{aligned}
\Psi^{(1)^{\prime \prime}}+4 \mathscr{H} \Psi^{(1)^{\prime}}-\frac{1}{3} \nabla^{2} \Psi^{(1)} & =\mathscr{H} Q^{(1)^{\prime}}+\frac{1}{3} \nabla^{2} Q^{(1)}, \\
\nabla^{2} Q^{(1)} & =\frac{9}{2} \mathscr{H}^{2} \frac{\partial_{i} \partial^{j}}{\nabla^{2}} \Pi_{T j}^{(1) i},
\end{aligned}
$$

where the total anisotropic stress tensor is

$$
\Pi_{T j}^{i}=\frac{\bar{\rho}_{\gamma}}{\bar{\rho}_{T}} \Pi_{\gamma j}^{i}+\frac{\bar{\rho}_{\nu}}{\bar{\rho}_{T}} \Pi_{\nu j}^{i} .
$$

We may safely neglect the quadrupole and solve (B.8) setting $u_{ \pm}=\Phi_{ \pm}^{(1)} \eta$. Then, (B.8), in Fourier space, becomes

$$
u^{\prime \prime}+\frac{2}{\eta} u^{\prime}+\left(\frac{k^{2}}{3}-\frac{2}{\eta^{2}}\right) u=0
$$

This equation has as independent solutions $u_{+}=j_{1}(k \eta / \sqrt{3})$, the spherical Bessel function of order 1 , and $u_{-}=n_{1}(\mathrm{k \eta} / \sqrt{3})$, the spherical Neumann function of order 1 . The latter blows up as $\eta$ gets small and we discard it on the basis of initial conditions. The final solution is therefore

$$
\begin{aligned}
\Phi_{k}^{(1)}= & 3 \Phi^{(1)}(0) \\
& \times \frac{\sin (k \eta / \sqrt{3})-(k \eta / \sqrt{3}) \cos (k \eta / \sqrt{3})}{(k \eta / \sqrt{3})^{3}}
\end{aligned}
$$

where $\Phi^{(1)}(0)$ represents the initial condition deep in the radiation era.

At the same order in perturbation theory, the radiation velocity can be read off from (30)

$$
v_{\gamma}^{(1) i}=-\frac{1}{2 \mathscr{H}^{2}} \frac{\left(a \partial^{i} \Phi^{(1)}\right)}{a} .
$$


At second order, combining (29), (34), we find

$$
\begin{aligned}
\Psi^{(2)^{\prime \prime}}+4 \mathscr{H} \Psi^{(2)^{\prime}}-\frac{1}{3} \nabla^{2} \Psi^{(2)}=S_{\gamma}, & \\
S_{\gamma}= & 4\left(\Psi^{(1)^{\prime}}\right)^{2}+2 \Phi^{(1)^{\prime}} \Psi^{(1)^{\prime}}+\frac{4}{3}\left(\Phi^{(1)}+\Psi^{(1)}\right) \nabla^{2} \Psi^{(1)} \\
& -\frac{2}{3}\left(\partial_{k} \Phi^{(1)} \partial^{k} \Phi^{(1)}+\partial_{k} \Psi^{(1)} \partial^{k} \Psi^{(1)}-\partial_{k} \Phi^{(1)} \partial^{k} \Psi^{(1)}\right) \\
& +\mathscr{H} Q^{(2)^{\prime}}+\frac{1}{3} \nabla^{2} Q^{(2)}+\frac{4}{3}\left(\Phi^{(1)}+\Psi^{(1)}\right) \nabla^{2} Q^{(1)}, \\
\frac{1}{2} \nabla^{2} Q^{(2)} & -\partial_{k} \Phi^{(1)} \partial^{k} \Psi^{(1)}-\frac{1}{2}\left(\partial_{k} \Phi^{(1)} \partial^{k} \Phi^{(1)}-\partial_{k} \Psi^{(1)} \partial^{k} \Psi^{(1)}\right) \\
& +3 \frac{\partial_{i} \partial^{j}}{\nabla^{2}}\left[\partial^{i} \Phi^{(1)} \partial_{j} \Psi^{(1)}+\frac{1}{2}\left(\partial^{i} \Phi^{(1)} \partial_{j} \Phi^{(1)}-\partial^{i} \Psi^{(1)} \partial_{j} \Psi^{(1)}\right)\right] \\
& +\frac{9}{2} \mathscr{H}^{2} \frac{\partial_{i} \partial^{j}}{\nabla^{2}} \frac{\Pi_{T j}^{(2) i}}{2}-9 \mathscr{H}^{2} \frac{\partial_{i} \partial^{j}}{\nabla^{2}}\left(\Psi^{(1)} \Pi_{T j}^{(1) i}\right),
\end{aligned}
$$

whose solution is

$$
\begin{aligned}
\Psi^{(2)}= & \Psi_{\text {hom. }}^{(2)} \\
& +\int_{0}^{\eta} d \eta^{\prime} \frac{\Phi_{+}^{(1)}(\eta) \Phi_{-}^{(1)}\left(\eta^{\prime}\right)-\Phi_{-}^{(1)}(\eta) \Phi_{+}^{(1)}\left(\eta^{\prime}\right)}{W\left(\eta^{\prime}\right)} S_{\gamma}\left(\eta^{\prime}\right),
\end{aligned}
$$

where $W(\eta)=(a(0) / a)^{4}$ is the Wronskian, and $\Psi_{\text {hom. }}^{(2)}$ is the solution of the homogeneous equation.

The equation of motion for the vector metric perturbations reads

$$
\begin{aligned}
& -\frac{1}{2} \nabla^{2} \omega^{i}+4 \mathscr{H}^{2} \omega^{i} \\
& =\left(\delta_{j}^{i}-\frac{\partial^{i} \partial_{j}}{\nabla^{2}}\right) \\
& \times\left[2 \Psi^{(1)^{\prime}} \partial^{j} \Phi^{(1)}+\mathscr{H}^{2} \frac{\bar{\rho}_{\gamma}+\bar{\rho}_{\nu}}{\bar{\rho}_{T}} \omega^{j}\right. \\
& \quad-2 \mathscr{H}^{2}\left(\frac{\bar{\rho}_{\gamma}}{\bar{\rho}_{T}} v_{\gamma}^{(2) j}+\frac{\bar{\rho}_{v}}{\bar{\rho}_{T}} v_{\nu}^{(2) i}+2 \frac{\bar{\rho}_{\gamma}}{\bar{\rho}_{T}} \Delta_{00}^{(1) \gamma} v_{\gamma}^{(1) j}\right. \\
& +2 \frac{\bar{\rho}_{\nu}}{\bar{\rho}_{T}} \Delta_{00}^{(1) v} v_{\nu}^{(1) j}+2\left(\Phi^{(1)}-\Psi^{(1)}\right) \frac{\bar{\rho}_{\gamma}}{\bar{\rho}_{T}} v_{\gamma}^{(1) j} \\
& \left.\left.+2\left(\Phi^{(1)}-\Psi^{(1)}\right) \frac{\bar{\rho}_{\nu}}{\bar{\rho}_{T}} v_{\nu}^{(1) j}\right)\right],
\end{aligned}
$$

where $\bar{\rho}_{T}$ is the total background energy density. The Einstein equations for a universe filled by CDM and a relativisitc component can be foun in [47].

\section{Linear Solution of the Boltzmann Equations}

In this section, we will solve the Boltzmann equations at first order in perturbation theory. The interested reader will find the extension of these formulae to second order in [47]. The first two moments of the photon Boltzmann equation are obtained by integrating (129) over $d \Omega_{n} / 4 \pi$ and $d \Omega_{n} n^{i} / 4 \pi$, respectively, and they lead to the density and velocity continuity equations

$$
\begin{gathered}
\Delta_{00}^{(1)^{\prime}}+\frac{4}{3} \partial_{i} v_{\gamma}^{(1) i}-4 \Psi^{(1)^{\prime}}=0 \\
v_{\gamma}^{(1) i^{\prime}}+\frac{3}{4} \partial_{j} \Pi_{\gamma}^{(1) j i}+\frac{1}{4} \Delta_{00}^{(1), i}+\Phi^{(1), i}=-\tau^{\prime}\left(v^{(1) i}-v_{\gamma}^{(1)}\right),
\end{gathered}
$$

where $\Pi^{i j}$ is the photon quadrupole moment, defined in (195).

Let us recall here that $\delta_{\gamma}^{(1)}=\Delta_{00}^{(1)}=\int d \Omega \Delta^{(1)} / 4 \pi$ and that the photon velocity is given by (186).

The two equations above are complemented by the momentum continuity equation for baryons, which can be conveniently written as

$$
v^{(1) i}=v_{\gamma}^{(1) i}+\frac{R}{\tau^{\prime}}\left[v^{(1) i^{\prime}}+\mathscr{H} v^{(1) i}+\Phi^{(1), i}\right]
$$

where we have introduced the baryon-photon ratio $R \equiv$ $3 \rho_{b} /\left(4 \rho_{\gamma}\right)$

Equation (C.3) is in a form ready for a consistent expansion in the small quantity $\tau^{-1}$ which can be performed in the tight-coupling limit. By first taking $v^{(1) i}=v_{\gamma}^{(1) i}$ at zero order and then using this relation in the left-hand side of (C.3) one obtains

$$
v^{(1) i}-v_{\gamma}^{(1) i}=\frac{R}{\tau^{\prime}}\left[v_{\gamma}^{(1) i^{\prime}}+\mathscr{H} v_{\gamma}^{(1) i}+\Phi^{(1), i}\right]
$$

Such an expression for the difference of velocities can be used in (C.2) to give the evolution equation for the photon velocity in the limit of tight coupling

$$
v_{\gamma}^{(1) i^{\prime}}+\mathscr{H} \frac{R}{1+R} v_{\gamma}^{(1) i}+\frac{1}{4} \frac{\Delta_{00}^{(1), i}}{1+R}+\Phi^{(1), i}=0 .
$$

Notice that in (C.5) we are neglecting the quadrupole of the photon distribution $\Pi^{(1) i j}$ (and all the higher moments) since it is well known that at linear order such moment(s) are suppressed in the tight-coupling limit by (successive powers of) $1 / \tau$ with respect to the first two moments, the photon energy density and velocity. Equations (C.1) and (C.5) are the master equations which govern the photon-baryon fluid acoustic oscillations before the epoch of recombination when photons and baryons are tightly coupled by Compton scattering. 
In fact, one can combine these two equations to get a single second-order differential equation for the photon energy density perturbations $\Delta_{00}^{(1)}$. Deriving (C.1) with respect to conformal time and using (C.5) to replace $\partial_{i} v_{\gamma}^{(1) i}$ yields

$$
\begin{aligned}
& \left(\Delta_{00}^{(1)^{\prime \prime}}-4 \Psi^{(1)^{\prime \prime}}\right)+\mathcal{H} \frac{R}{1+R}\left(\Delta_{00}^{(1)^{\prime}}-4 \Psi^{(1)^{\prime}}\right) \\
& \quad-c_{s}^{2} \nabla^{2}\left(\Delta_{00}^{(1)}-4 \Psi^{(1)}\right)=\frac{4}{3} \nabla^{2}\left(\Phi^{(1)}+\frac{\Psi^{(1)}}{1+R}\right)
\end{aligned}
$$

where $c_{s}=1 / \sqrt{3(1+R)}$ is the speed of sound of the photonbaryon fluid. Indeed, in order to solve (C.6) one needs to know the evolution of the gravitational potentials. We will come back later to the discussion of the solution of (C.6).

A useful relation is obtained by considering the continuity equation for the baryon density perturbation. By perturbing at first order (176), we obtain

$$
\delta_{b}^{(1)^{\prime}}+v_{, i}^{i}-3 \Psi^{(1)^{\prime}}=0 .
$$

Subtracting (C.7) form (C.1) brings

$$
\Delta_{00}^{(1)^{\prime}}-\frac{4}{3} \delta_{b}^{(1)^{\prime}}+\frac{4}{3}\left(v_{\gamma}^{(1) i}-v^{(1) i}\right)_{, i}=0
$$

which implies that at lowest order in the tight-coupling approximation

$$
\Delta_{00}^{(1)}=\frac{4}{3} \delta_{b}^{(1)},
$$

for adiabatic perturbations.

C.1. Linear Solutions in the Limit of Tight Coupling. In this section, we briefly recall how to obtain at linear order the solutions of the Boltzmann equations (C.6). These correspond to the acoustic oscillations of the photon-baryon fluid for modes which are within the horizon at the time of recombination. It is well known that, in the variable $\left(\Delta_{00}^{(1)}-\right.$ $\left.4 \Psi^{(1)}\right)$, the solution can be written as $[73,74]$

$$
\begin{aligned}
{[1} & +R(\eta)]^{1 / 4}\left(\Delta_{00}^{(1)}-4 \Psi^{(1)}\right) \\
= & A \cos \left[k r_{s}(\eta)\right]+B \sin \left[k r_{s}(\eta)\right] \\
& -4 \frac{k}{\sqrt{3}} \int_{0}^{\eta} d \eta^{\prime}\left[1+R\left(\eta^{\prime}\right)\right]^{3 / 4}\left(\Phi^{(1)}\left(\eta^{\prime}\right)+\frac{\Psi^{(1)}\left(\eta^{\prime}\right)}{1+R\left(\eta^{\prime}\right)}\right) \\
& \times \sin \left[k\left(r_{s}(\eta)-r_{s}\left(\eta^{\prime}\right)\right)\right],
\end{aligned}
$$

where the sound horizon is given by $r_{s}(\eta)=\int_{0}^{\eta} d \eta^{\prime} c_{s}\left(\eta^{\prime}\right)$, with $R=3 \rho_{b} /\left(4 \rho_{\gamma}\right)$. The constants $A$ and $B$ in (C.10) are fixed by the choice of initial conditions.
In order to give an analytical, we will use some simplifications following [70, 99]. First, for simplicity, we are going to neglect the ratio $R$ wherever it appears, except in the arguments of the varying cosines and sines, where we will treat $R=R_{*}$ as a constant evaluated at the time of recombination. In this way, we keep track of a damping of the photon velocity amplitude with respect to the case $R=0$ which prevents the acoustic peaks in the powerspectrum to disappear. Treating $R$ as a constant is justified by the fact that for modes within the horizon the time scale of the oscillations is much shorter than the time scale on which $R$ varies. If $R$ is a constant the sound speed is just a constant $c_{s}=1 / \sqrt{3\left(1+R_{*}\right)}$, and the sound horizon is simply $r_{s}(\eta)=c_{s} \eta$.

Second, we are going to solve for the evolutions of the perturbations in two well distinguished limiting regimes. One regime is for those perturbations which enter the Hubble radius when matter is the dominant component, that is at times much bigger than the equality epoch, with $k \ll k_{\mathrm{eq}} \sim \eta_{\mathrm{eq}}^{-1}$, where $k_{\mathrm{eq}}$ is the wavenumber of the Hubble radius at the equality epoch. The other regime is for those perturbations with much smaller wavelengths which enter the Hubble radius when the Universe is still radiation dominated, that is perturbations with wavenumbers $k \gg$ $k_{\mathrm{eq}} \sim \eta_{\mathrm{eq}}^{-1}$. In fact, we are interested in perturbation modes which are within the horizon by the time of recombination $\eta_{*}$. Therefore, we will further suppose that $\eta_{*} \gg \eta_{\mathrm{eq}}$ in order to study such modes in the first regime. Even though $\eta_{*} \gg \eta_{\mathrm{eq}}$ is not the real case, it allows to obtain some analytical expressions.

Before solving for these two regimes let us fix our initial conditions, which are taken on large scales deep in the radiation dominated era (for $\eta \rightarrow 0$ ). During this epoch, for adiabatic perturbations, the gravitational potentials remain constant on large scales (we are neglecting anisotropic stresses so that $\left.\Phi^{(1)} \simeq \Psi^{(1)}\right)$ and from the $(0-0)$-component of Einstein equations

$$
\Phi^{(1)}(0)=-\frac{1}{2} \Delta_{00}^{(1)}(0)
$$

On the other hand, from the energy continuity equation (C.1) on large scales

$$
\Delta_{00}^{(1)}-4 \Psi^{(1)}=\text { const.; }
$$

from (C.11) the constant on the right-hand side of (C.12) is fixed to be $-6 \Phi^{(1)}(0)$; thus we find $B=0$ and $A=-6 \Phi^{(1)}(0)$.

With our simplifications, (C.10) then reads

$$
\begin{aligned}
\Delta_{00}^{(1)}-4 \Psi^{(1)}= & -6 \Phi^{(1)}(0) \cos \left(\omega_{0} \eta\right) \\
& -\frac{8 k}{\sqrt{3}} \int_{0}^{\eta} d \eta^{\prime} \Phi^{(1)}\left(\eta^{\prime}\right) \sin \left[\omega_{0}\left(\eta-\eta^{\prime}\right)\right],
\end{aligned}
$$

where $\omega_{0}=k c_{s}$. 
C.2. Perturbation Modes with $k \gg k_{\text {eq. }}$. This regime corresponds to perturbation modes which enter the Hubble radius when the universe is matter dominated at times $\eta \gg \eta_{\text {eq }}$. During matter domination, the gravitational potential remains constant (both on superhorizon and subhorizon scales), as one can see for example from (B.1), and its value is fixed to $\Phi^{(1)}(k, \eta)=(9 / 10) \Phi^{(1)}(0)$, where $\Phi^{(1)}(0)$ corresponds to the gravitational potential on large scales during the radiation dominated epoch. Since we are interested in the photon anisotropies around the time of recombination, when matter is dominating, we can perform the integral appearing in (C.10) by taking the gravitational potential equal to its value during matter domination so that it is easily computed

$$
\begin{aligned}
2 \int_{0}^{\eta} d \eta^{\prime} \Phi^{(1)}\left(\eta^{\prime}\right) \sin \left[\omega_{0}\left(\eta-\eta^{\prime}\right)\right] \\
=\frac{18}{10} \frac{\Phi^{(1)}(0)}{\omega_{0}}\left(1-\cos \left(\omega_{0} \eta\right)\right) .
\end{aligned}
$$

Thus, (C.13) gives

$$
\Delta_{00}^{(1)}-4 \Psi^{(1)}=\frac{6}{5} \Phi^{(1)}(0) \cos \left(\omega_{0} \eta\right)-\frac{36}{5} \Phi^{(1)}(0) .
$$

The baryon-photon fluid velocity can then be obtained as $\partial_{i} v_{\gamma}^{(1) i}=-3\left(\Delta_{00}^{(1)}-4 \Psi^{(1)}\right)^{\prime} / 4$ from (C.1). In Fourier space

$$
i k_{i} v_{\gamma}^{(1) i}=\frac{9}{10} \Phi^{(1)}(0) \sin \left(\omega_{0} \eta\right) \omega_{0}
$$

where, going to Fourier space, $\partial_{i} v_{\gamma}^{(1) i} \rightarrow i k_{i} v_{\gamma}^{(1) i}(\mathbf{k})$ and

$$
v_{\gamma}^{(1) i}=-i \frac{k^{i}}{k} \frac{9}{10} \Phi^{(1)}(0) \sin \left(\omega_{0} \eta\right) c_{s}
$$

since the linear velocity is irrotational.

Notice that under the approximations that $R=$ const. and $\Phi^{(1)}=\Psi^{(1)}=$ const., it is easy to find a more accurate solution to (C.6) which better accounts for the presence of the baryons (giving rise to the so called baryon-drag effect, as clearly explained in $[73,74,95])$

$$
\frac{\Delta_{00}^{(1)}}{4}=\left[\frac{\Delta_{00}^{(1)}}{4}(0)+(1+R) \Phi^{(1)}\right] \cos \left(k r_{s}\right)-(1+R) \Phi^{(1)} .
$$

C.3. Perturbation Modes with $k \gg k_{\mathrm{eq}}$. This regime corresponds to perturbation modes which enter the Hubble radius when the universe is still radiation dominated at times $\eta \ll \eta_{\text {eq }}$. In this case an approximate analytical solution for the evolution of the perturbations can be obtained by considering the gravitational potential for a pure radiation dominated epoch, given by (B.11). For the integral in (C.13), we thus find

$$
\int_{0}^{\eta} \Phi^{(1)}\left(\eta^{\prime}\right) \sin \left[\omega_{0}\left(\eta-\eta^{\prime}\right)\right]=-\frac{3}{2 \omega_{0}} \cos \left(\omega_{0} \eta\right)
$$

where we have kept only the dominant contribution oscillating in time, while neglecting terms which decay in time. The solution (C.13) becomes

$$
\Delta_{00}^{(1)}-4 \Psi^{(1)}=6 \Phi^{(1)}(0) \cos \left(\omega_{0} \eta\right),
$$

and the velocity is given by

$$
v_{\gamma}^{(1) i}=-i \frac{k^{i}}{k} \frac{9}{2} \Phi^{(1)}(0) \sin \left(\omega_{0} \eta\right) c_{s}
$$

Notice that the solutions (C.20)-(C.21) are actually correct only when radiation dominates. Indeed, between the epoch of equality and recombination, matter starts to dominate. Full account of such a period is given for example, in Section 7.3 of [70], while its consequences for the CMB anisotropy evolution can be found for example, in [100]. We refer to [47], where an alternative way to solve for the acoustic oscillations in this regime is displayed which turns out to be useful for the corresponding computation at second order in Section 7.5.

\section{Acknowledgments}

A. Riotto is on leave of absence from INFN, Sezione di Padova. This research has been partially supported by the ASI contract I/016/07/0 "COFIS", the ASI/INAF Agreement I/072/09/0 for the Planck LFI Activity of Phase E2. A. Riotto acknowledges support by the EU Marie Curie Network UniverseNet (HPRNCT2006035863). The authors would like to thank Michele Liguori for stimulating discussions, and Daisuke Nitta and Eiichiro Komatsu for past and ongoing enjoyable collaborations.

\section{References}

[1] D. H. Lyth and A. Riotto, "Particle physics models of inflation and the cosmological density perturbation," Physics Report, vol. 314, no. 1-2, pp. 1-146, 1999.

[2] J. Dunkley, E. Komatsu, M. R. Nolta et al., "Five-year wilkinson microwave anisotropy probe observations: likelihoods and parameters from the WMAP data," Astrophysical Journal, Supplement Series, vol. 180, no. 2, pp. 306-329, 2009.

[3] http://planck.esa.int/.

[4] D. Baumann, M. G. Jackson, P. Adshead et al., "Probing inflation with CMB polarization," in CMB Polarization Workshop: Theory and Foregrounds, vol. 1141 of AIP Conference Proceedings, pp. 10-120, 2009.

[5] N. Bartolo, E. Komatsu, S. Matarrese, and A. Riotto, "NonGaussianity from inflation: theory and observations," Physics Reports, vol. 402, no. 3-4, pp. 103-266, 2004.

[6] V. Acquaviva, N. Bartolo, S. Matarrese, and A. Riotto, "Gauge-invariant second-order perturbations and nonGaussianity from inflation," Nuclear Physics B, vol. 667, no. 1-2, pp. 119-148, 2003.

[7] J. Maldacena, "Non-Gaussian features of primordial fluctuations in single field inflationary models ," Journal of High Energy Physics, vol. 2003, no. 5, article 013, 2003.

[8] N. Bartolo, S. Matarrese, and A. Riotto, "Non-Gaussianity from inflation," Physical Review D, vol. 65, no. 10, Article ID 103505, 2002. 
[9] F. Bernardeau and J.-P. Uzan, "Non-Gaussianity in multifield inflation," Physical Review D, vol. 66, no. 10, Article ID 103506, 2002.

[10] D. H. Lyth, C. Ungarelli, and D. Wands, "Primordial density perturbation in the curvaton scenario," Physical Review D, vol. 67, no. 2, Article ID 023503, 2003.

[11] T. Hamazaki and H. Kodama, "Evolution of cosmological perturbations during reheating," Progress of Theoretical Physics, vol. 96, no. 6, pp. 1123-1145, 1996.

[12] G. Dvali, A. Gruzinov, and M. Zaldarriaga, "New mechanism for generating density perturbations from inflation," Physical Review D, vol. 69, no. 2, Article ID 023505, 2004.

[13] L. Kofman, "Probing string theory with modulated cosmological fluctuations," http://arxiv.org/abs/astro-ph/0303614.

[14] G. Dvali, A. Gruzinov, and M. Zaldarriaga, "Cosmological perturbations from inhomogeneous reheating, freeze-out, and mass domination," Physical Review D, vol. 69, no. 8, Article ID 083505, 2004.

[15] C. W. Bauer, M. L. Graesser, and M. P. Salem, "Fluctuating annihilation cross sections and the generation of density perturbations," Physical Review D, vol. 72, no. 2, Article ID 023512, 7 pages, 2005.

[16] D. H. Lyth, "Generating the curvature perturbation at the end of inflation," Journal of Cosmology and Astroparticle Physics, vol. 2005, no. 11, article 006, pp. 111-120, 2005.

[17] M. P. Salem, "Generation of density perturbations at the end of inflation," Physical Review D, vol. 72, no. 12, Article ID 123516, 7 pages, 2005.

[18] D. H. Lyth and A. Riotto, "Generating the curvature perturbation at the end of inflation in string theory," Physical Review Letters, vol. 97, no. 12, Article ID 121301, 2006.

[19] M. Bastero-Gil, V. Di Clemente, and S. F. King, "Preheating curvature perturbations with a coupled curvaton," Physical Review D, vol. 70, no. 2, Article ID 023501, 2004.

[20] E. W. Kolb, A. Riotto, and A. Vallinotto, "Curvature perturbations from broken symmetries," Physical Review D, vol. 71, no. 4, Article ID 043513, 10 pages, 2005.

[21] C. T. Byrnes and D. Wands, "Scale-invariant perturbations from chaotic inflation," Physical Review D, vol. 73, no. 6, Article ID 063509, 8 pages, 2006.

[22] T. Matsuda, “Topological curvatons," Physical Review D, vol. 72, no. 12, Article ID 123508, 8 pages, 2005.

[23] M. Alishahiha, E. Silverstein, and D. Tong, "DBI in the sky: non-Gaussianity from inflation with a speed limit," Physical Review D, vol. 70, no. 12, Article ID 123505, 2004.

[24] R. Holman and A. J. Tolley, "Enhanced non-Gaussianity from excited initial states," Journal of Cosmology and Astroparticle Physics, vol. 2008, no. 5, article 001, 2008.

[25] D. Babich, P. Creminelli, and M. Zaldarriaga, "The shape of non-Gaussianities," Journal of Cosmology and Astroparticle Physics, vol. 2004, no. 8, article 009, pp. 199-217, 2004.

[26] J. R. Fergusson and E. P. S. Shellard, "Shape of primordial non-Gaussianity and the CMB bispectrum," Physical Review D, vol. 80, no. 4, Article ID 043510, 2009.

[27] W. Hu, "Angular trispectrum of the cosmic microwave background," Physical Review D, vol. 64, no. 8, Article ID 083005, 2001.

[28] T. Okamoto and W. Hu, "Angular trispectra of CMB temperature and polarization," Physical Review D, vol. 66, no. 6, Article ID 063008, 2002.
[29] G. De Troia, P. A. R. Ade, J. J. Bock et al., "The trispectrum of the cosmic microwave background on subdegree angular scales: an analysis of the BOOMERanG data," Monthly Notices of the Royal Astronomical Society, vol. 343, no. 1, pp. 284-292, 2003.

[30] J. Lesgourgues, M. Liguori, S. Matarrese, and A. Riotto, "CMB lensing extraction and primordial non-Gaussianity," Physical Review D, vol. 71, no. 10, Article ID 103514, 2005.

[31] N. Bartolo, S. Matarrese, and A. Riotto, "The full secondorder radiation transfer function for large-scale CMB anisotropies," Journal of Cosmology and Astroparticle Physics, vol. 2006, no. 5, article 010, 2006.

[32] T. Pyne and S. M. Carroll, "Higher-order gravitational perturbations of the cosmic microwave background," Physical Review D, vol. 53, no. 6, pp. 2920-2929, 1996.

[33] N. Bartolo, S. Matarrese, and A. Riotto, "Enhancement of non-Gaussianity after inflation," Journal of High Energy Physics, vol. 2004, no. 4, article 006, 2004.

[34] N. Bartolo, S. Matarrese, and A. Riotto, "Gauge-invariant temperature anisotropies and primordial non-Gaussianity," Physical Review Letters, vol. 93, no. 23, Article ID 231301, 2004.

[35] K. Tomita, "Relativistic second-order perturbations of nonzero- $\Lambda$ flat cosmological models and CMB anisotropies," Physical Review D, vol. 71, no. 8, Article ID 083504, 11 pages, 2005.

[36] N. Bartolo, S. Matarrese, and A. Riotto, "Non-Gaussianity of large-scale cosmic microwave background anisotropies beyond perturbation theory," Journal of Cosmology and Astroparticle Physics, vol. 2005, no. 8, pp. 177-196, 2005.

[37] L. Boubekeur, P. Creminelli, G. D'Amico, J. Norea, and F. Vernizzi, "Sachs-Wolfe at second order: the CMB bispectrum on large angular scales," Journal of Cosmology and Astroparticle Physics, vol. 2009, no. 8, article 029, 2009.

[38] G. D'Amico, N. Bartolo, S. Matarrese, and A. Riotto, “CMB temperature anisotropies from third-order gravitational perturbations," Journal of Cosmology and Astroparticle Physics, vol. 2008, no. 1, article 005, 2008.

[39] W. Hu and S. Dodelson, "Cosmic microwave background anisotropies," Annual Review of Astronomy and Astrophysics, vol. 40, pp. 171-216, 2002.

[40] U. Seljak and M. Zaldarriaga, "Direct signature of an evolving gravitational potential from the cosmic microwave background," Physical Review D, vol. 60, no. 4, Article ID 043504, 4 pages, 1999.

[41] D. M. Goldberg and D. N. Spergel, "Microwave background bispectrum. II. A probe of the low redshift universe," Physical Review D, vol. 59, no. 10, Article ID 103002, 6 pages, 1999.

[42] D. M. Goldberg and D. N. Spergel, "Microwave background bispectrum. I. Basic formalism," Physical Review D, vol. 59, no. 10, Article ID 103002, 8 pages, 1999.

[43] E. Komatsu and D. N. Spergel, "Acoustic signatures in the primary microwave background bispectrum," Physical Review D, vol. 63, no. 6, Article ID 063002, 2001.

[44] K. M. Smith and M. Zaldarriaga, "Algorithms for bispectra: forecasting, optimal analysis, and simulation," http://arxiv.org/abs/astro-ph/0612571.

[45] P. Creminelli and M. Zaldarriaga, "CMB 3-point functions generated by nonlinearities at recombination," Physical Review D, vol. 70, no. 8, Article ID 083532, 2004.

[46] N. Bartolo, S. Matarrese, and A. Riotto, "Cosmic microwave background anisotropies at second order: I," Journal of Cosmology and Astroparticle Physics, vol. 2006, no. 6, article 024, 2006. 
[47] N. Bartolo, S. Matarrese, and A. Riotto, "CMB anisotropies at second-order II: analytical approach," Journal of Cosmology and Astroparticle Physics, vol. 2007, no. 1, article 019, 2007.

[48] C. Pitrou, J.-P. Uzan, and F. Bernardeau, "Cosmic microwave background bispectrum on small angular scales," Physical Review D, vol. 78, no. 6, Article ID 063526, 2008.

[49] C. Pitrou, "The radiative transfer at second order: a full treatment of the Boltzmann equation with polarization," Classical and Quantum Gravity, vol. 26, no. 6, Article ID 065006, 2009.

[50] C. Pitrou, "The radiative transfer for polarized radiation at second order in cosmological perturbations," General Relativity and Gravitation, vol. 41, no. 11, pp. 2587-2595, 2009.

[51] S. Matarrese, S. Mollerach, and M. Bruni, "Relativistic second-order perturbations of the Einstein-de Sitter universe," Physical Review D, vol. 58, no. 4, Article ID 043504, 1998.

[52] D. S. Salopek and J. R. Bond, "Nonlinear evolution of long-wavelength metric fluctuations in inflationary models," Physical Review D, vol. 42, no. 12, pp. 3936-3962, 1990.

[53] E. W. Kolb, S. Matarrese, A. Notari, and A. Riotto, "Cosmological influence of super-Hubble perturbations," Modern Physics Letters A, vol. 20, no. 35, pp. 2705-2710, 2005.

[54] K. A. Malik and D. Wands, "Evolution of second-order cosmological perturbations," Classical and Quantum Gravity, vol. 21, no. 11, pp. L65-L71, 2004.

[55] N. Bartolo, S. Matarrese, and A. Riotto, "Non-Gaussianity in the curvaton scenario," Physical Review D, vol. 69, no. 4, Article ID 043503, 2004.

[56] N. Arkani-Hamed, P. Creminelli, S. Mukohyama, and M. Zaldarriaga, "Ghost inflation," Journal of Cosmology and Astroparticle Physics, vol. 2004, no. 4, article 001, pp. 1-18, 2004.

[57] K. M. Smith, L. Senatore, and M. Zaldarriaga, "Optimal limits on $f_{N L}$ local from WMAP 5-year data," Journal of Cosmology and Astroparticle Physics, vol. 2009, no. 9, article 006, 2009.

[58] L. Senatore, K. M. Smith, and M. Zaldarriaga, "NonGaussianities in single field inflation and their optimal limits from the WMAP 5-year data," Journal of Cosmology and Astroparticle Physics, vol. 2010, no. 1, article 028, 2010.

[59] S. Mollerach and S. Matarrese, "Cosmic microwave background anisotropies from second order gravitational perturbations," Physical Review D, vol. 56, no. 8, pp. 4494-4502, 1997.

[60] K. N. Ananda, C. Clarkson, and D. Wands, "Cosmological gravitational wave background from primordial density perturbations," Physical Review D, vol. 75, no. 12, Article ID 123518, 2007.

[61] D. Baumann, P. Steinhardt, K. Takahashi, and K. Ichiki, "Gravitational wave spectrum induced by primordial scalar perturbations," Physical Review D, vol. 76, no. 8, Article ID 084019, 2007.

[62] A. Mangilli, N. Bartolo, S. Matarrese, and A. Riotto, "Impact of cosmic neutrinos on the gravitational-wave background," Physical Review D, vol. 78, no. 8, Article ID 083517, 2008.

[63] L. Senatore, S. Tassev, and M. Zaldarriaga, "Cosmological perturbations at second order and recombination perturbed," Journal of Cosmology and Astroparticle Physics, vol. 2009, no. 8, article 031, 2009.

[64] S. Dodelson and J. M. Jubas, "Reionization and its imprint on the cosmic microwave background," Astrophysical Journal, vol. 439, no. 2, pp. 503-516, 1995.
[65] W. Hu, D. Scott, and J. Silk, "Reionization and cosmic microwave background distortions: a complete treatment of second-order Compton scattering," Physical Review D, vol. 49, no. 2, pp. 648-670, 1994.

[66] C. Pitrou, F. Bernardeau, and J. P. Uzan, “ The y-sky: diffuse spectral distortions of the cosmic microwave background," http://arxiv.org/abs/0912.3655.

[67] W. Hu, U. Seljak, M. White, and M. Zaldarriaga, "Complete treatment of CMB anisotropies in a FRW universe," Physical Review D, vol. 57, no. 6, pp. 3290-3301, 1998.

[68] W. $\mathrm{Hu}$ and M. White, "CMB anisotropies: total angular momentum method," Physical Review D, vol. 56, no. 2, pp. 596-615, 1997.

[69] W. Hu, "Reionization revisited: secondary cosmic microwave background anisotropies and polarization," Astrophysical Journal, vol. 529, pp. 12-25, 2000.

[70] S. Dodelson, Modern Cosmology, Academic Press, Amsterdam, The Netherlands, 2003.

[71] E. Sefusatti, M. Liguori, A. P. S. Yadav, M. G. Jackson, and E. Pajer, "Constraining running non-Gaussianity," Journal of Cosmology and Astroparticle Physics, vol. 2009, no. 12, article 022, 2009.

[72] E. Komatsu, J. Dunkley, M. R. Nolta et al., "Five-year wilkinson microwave anisotropy probe observations: cosmological interpretation," Astrophysical Journal, Supplement Series, vol. 180, no. 2, pp. 330-376, 2009.

[73] W. T. Hu, Wandering in the background: a CMB explorer, Ph.D. thesis, University of California at Berkeley, Berkeley, Calif, USA, 1995.

[74] W. Hu and N. Sugiyama, "Small-scale cosmological perturbations: an analytic approach," Astrophysical Journal, vol. 471, no. 2, pp. 542-570, 1996.

[75] N. Bartolo, S. Matarrese, and A. Riotto, "Evolution of secondorder cosmological perturbations and non-gaussianity," Journal of Cosmology and Astroparticle Physics, vol. 2004, 2004.

[76] A. Cooray and W. Hu, "Imprint of reionization on the cosmic microwave background bispectrum," Astrophysical Journal, vol. 534, no. 2, pp. 533-550, 2000.

[77] S. Donzelli, F. K. Hansen, M. Liguori, and D. Maino, "Impact of the 1/f noise and the asymmetric beam on nonGaussianity searches with planck," Astrophysical Journal, vol. 706, no. 2, pp. 1226-1240, 2009.

[78] P. Serra and A. Cooray, "Impact of secondary nonGaussianities on the search for primordial non-Gaussianity with CMB maps," Physical Review D, vol. 77, no. 10, Article ID 107305, 2008.

[79] D. Babich and M. Zaldarriaga, "Primordial bispectrum information from CMB polarization," Physical Review D, vol. 70, no. 8, Article ID 083005, 2004.

[80] D. Hanson, K. M. Smith, A. Challinor, and M. Liguori, "CMB lensing and primordial non-Gaussianity," Physical Review D, vol. 80, no. 8, Article ID 083004, 2009.

[81] A. Cooray, D. Sarkar, and P. Serra, "Weak lensing of the primary CMB bispectrum," Physical Review D, vol. 77, no. 12, Article ID 123006, 2008.

[82] A. Mangilli and L. Verde, "Non-Gaussianity and the CMB bispectrum: confusion between primordial and lensingRees-Sciama contribution?" Physical Review D, vol. 80, no. 12, Article ID 123007, 2009.

[83] D. Babich and E. Pierpaoli, "Point source contamination in CMB non-Gaussianity analyses," Physical Review D, vol. 77, no. 12, Article ID 123011, 2008. 
[84] A. Cooray, "Large scale pressure fluctuations and the Sunyaev-Zel'dovich effect," Physical Review D, vol. 62, no. 10, Article ID 103506, 15 pages, 2000.

[85] P. G. Castro, "The bispectrum and the trispectrum of the ostriker and vishniac effect," Physical Review D, vol. 67, Article ID 044039, 2004.

[86] R. Khatri and B. D. Wandelt, "O-V-S-Z and friends: nonGaussianity from inhomogeneous reionization," Astrophysical Journal, vol. 711, no. 2, pp. 1310-1315, 2010.

[87] R. Khatri and B. D. Wandelt, "Crinkles in the last scattering surface: non-Gaussianity from inhomogeneous recombination," Physical Review D, vol. 79, no. 2, Article ID 023501, 2009.

[88] R. Khatri and B. D. Wandelt, "More on crinkles in the last scattering surface," Physical Review D, vol. 81, no. 10, Article ID 103518, 2010.

[89] L. Senatore, S. Tassev, and M. Zaldarriaga, "Nongaussianities from perturbing recombination," Journal of Cosmology and Astroparticle Physics, vol. 2009, no. 9, article 038, 2009.

[90] L. Verde and D. N. Spergel, "Dark energy and cosmic microwave background bispectrum," Physical Review D, vol. 65, no. 4, Article ID 043007, 2002.

[91] N. Aghanim, S. Majumdar, and J. Silk, "Secondary anisotropies of the CMB," Reports on Progress in Physics, vol. 71, no. 6, Article ID 066902, 2008.

[92] E. Komatsu et al., "Non-Gaussianity as a probe of the physics of the primordial universe and the astrophysics of the low redshift universe," http://arxiv.org/abs/0902.4759.

[93] P. Creminelli, A. Nicolis, L. Senatore, M. Tegmark, and M. Zaldarriaga, "Limits on non-Gaussianities from WMAP data," Journal of Cosmology and Astroparticle Physics, vol. 2006, no. 5, article 004, 2006.

[94] W. Hu, "Weak lensing of the CMB: a harmonic approach," Physical Review D, vol. 62, no. 4, Article ID 043007, 17 pages, 2000.

[95] W. Hu and M. White, "The damping tail of cosmic microwave background anisotropies," Astrophysical Journal, vol. 479, no. 2, pp. 568-579, 1997.

[96] D. Nitta, E. Komatsu, N. Bartolo, S. Matarrese, and A. Riotto, "CMB anisotropies at second order III: bispectrum from products of the first-order perturbations," Journal of Cosmology and Astroparticle Physics, vol. 2009, no. 5, article 014, 2009.

[97] D. Nitta, N. Bartolo, S. Matarrese, and A. Riotto, to appear.

[98] M. Liguori, F. K. Hansen, E. Komatsu, S. Matarrese, and A. Riotto, "Testing primordial non-Gaussianity in CMB anisotropies," Physical Review D, vol. 73, no. 4, Article ID 043505, 12 pages, 2006.

[99] M. Zaldarriaga and D. D. Harari, "Analytic approach to the polarization of the cosmic microwave background in flat and open universes," Physical Review D, vol. 52, no. 6, pp. 3276 3287, 1995.

[100] V. Mukhanov, “"cMB-Slow” or how to determine cosmological parameters by hand?" International Journal of Theoretical Physics, vol. 43, no. 3, pp. 623-668, 2004. 

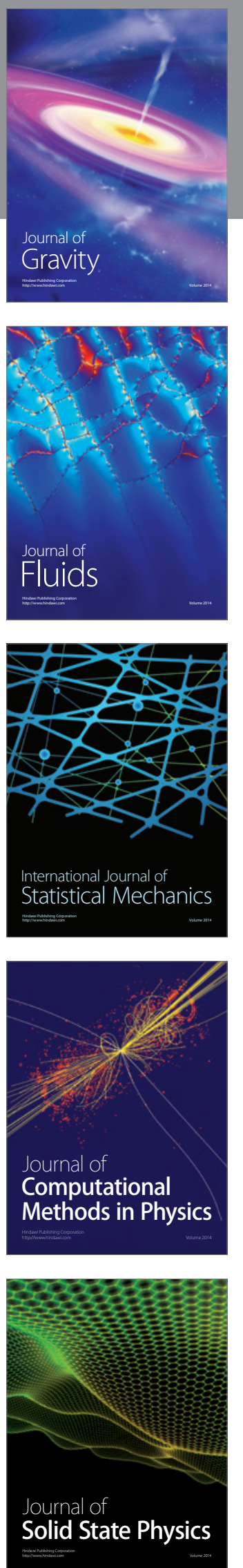

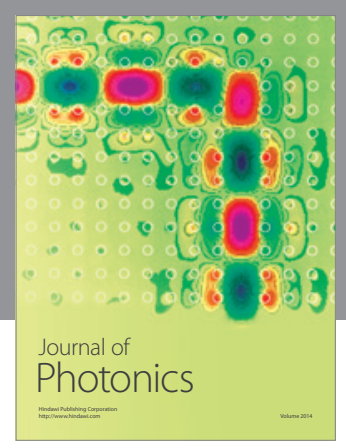

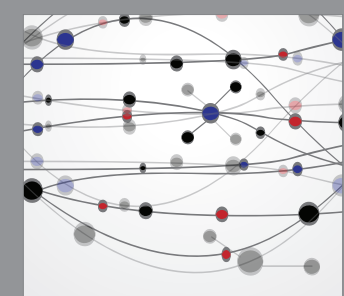

The Scientific World Journal
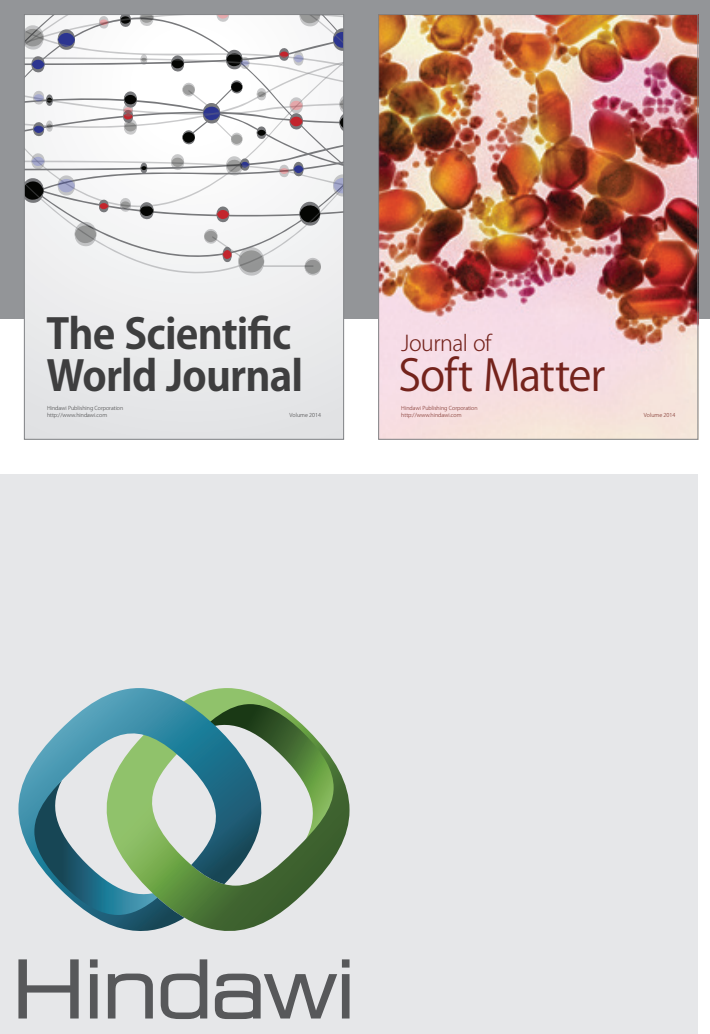

Submit your manuscripts at

http://www.hindawi.com
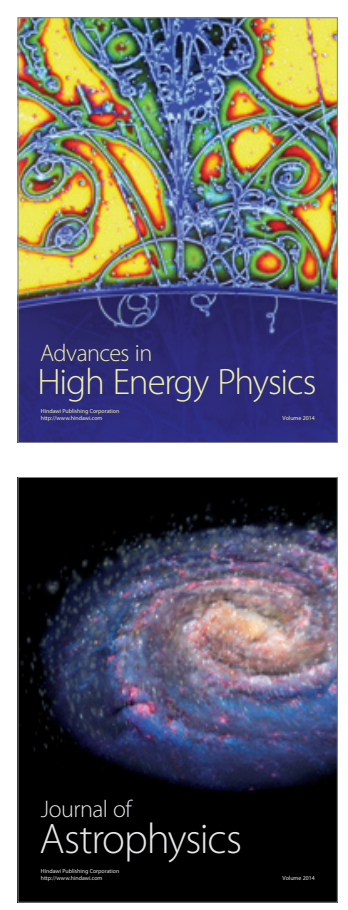
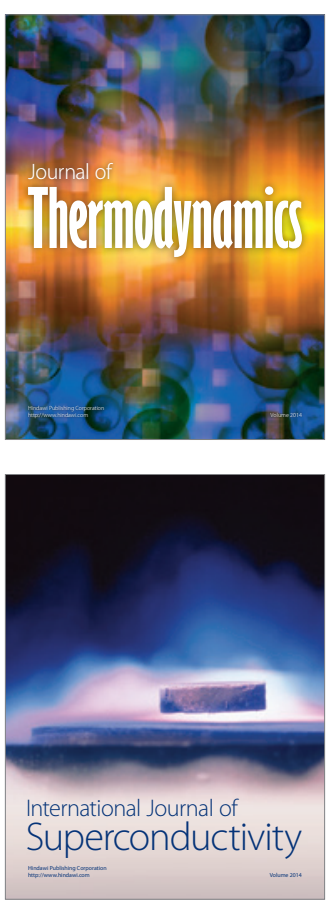
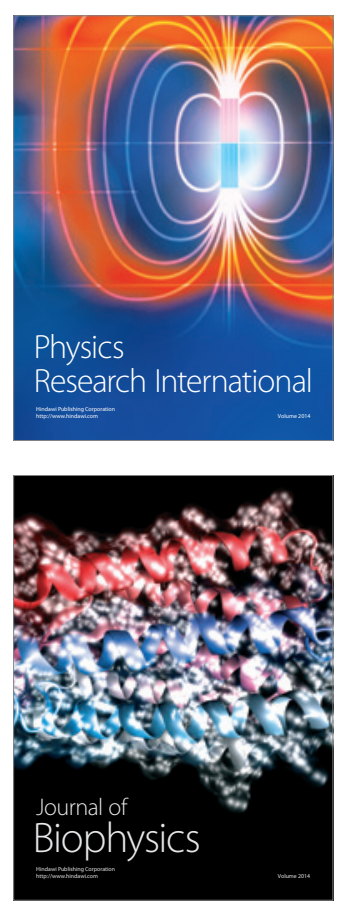
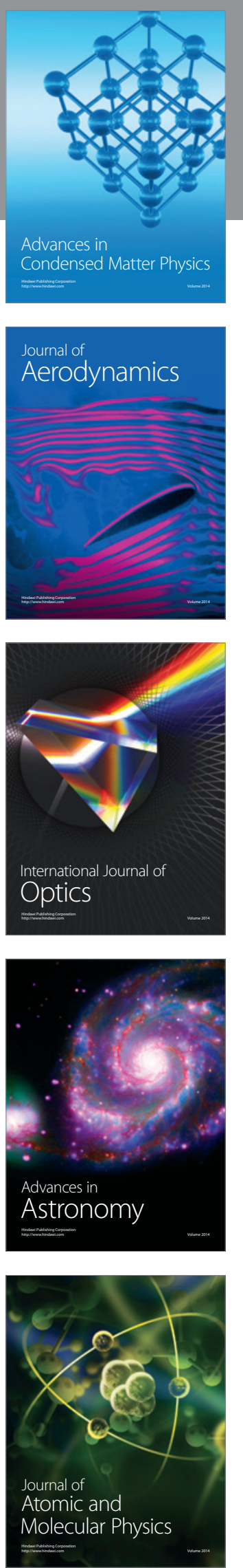